\title{
INVESTIGATION OF THE EFFECT OF IN-PLANE FIBER WAVINESS IN COMPOSITE MATERIALS THROUGH MULTIPLE SCALES OF TESTING AND FINITE ELEMENT MODELING
}

by

Michael William Lerman

A thesis submitted in partial fulfillment of the requirements for the degree

\author{
of \\ Master of Science \\ in \\ Mechanical Engineering \\ MONTANA STATE UNIVERSITY \\ Bozeman, Montana
}

April 2015 


\section{CCOPYRIGHT}

by

Michael William Lerman

2015

All Rights Reserved 


\section{ACKNOWLEDGEMENTS}

Many people have helped me through this process and I would first like to thank my committee of Dr. Douglas Cairns, Dr. Jared Nelson, Dr. David Miller, and Dr. Erick Johnson. I would like to highlight the contribution of Dr. Jared Nelson whose guidance along the way has been indispensable. I would like to thank Dan Samborsky and MSUCG for assistance with the experimental portion of this work. The modeling portion of this work could not have been achieved without the help of Dr. Ladean McKittrick. I would like to thank my fellow graduate students, especially Matt Peterson for his help with becoming proficient in Abaqus. Undergraduate students who have been of great assistance include Jocelyn Thompson, Nathan Fritz, and Carl Stringer. I would also like to thank Dr. David Klumpar for introducing me to Montana State University and encouraging me to pursue graduate school.

Lastly, I would like to thank those who have helped me be in a position to achieve a Master's degree as well as helped me succeed throughout this process. This includes my mom, my sister, my grandma, my girlfriend, my friends, and my pup. 
TABLE OF CONTENTS

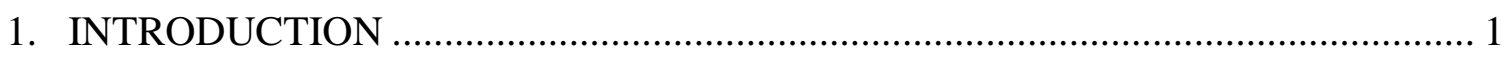

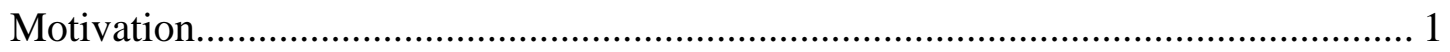

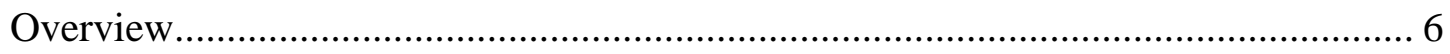

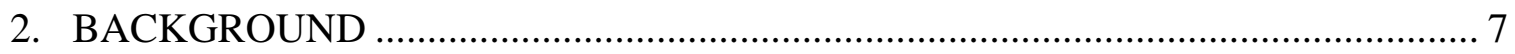

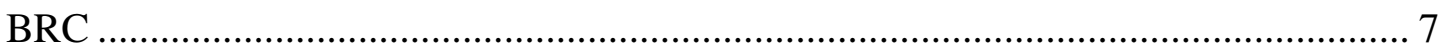

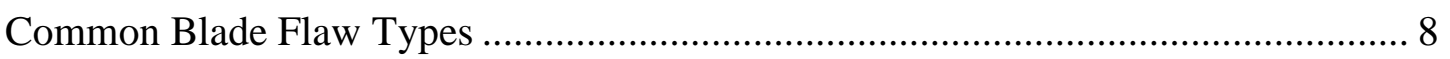

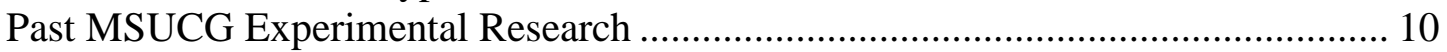

Composite Modeling Approaches ..................................................................... 15

Continuum Damage Modeling .............................................................. 17

Discrete Damage Modeling.................................................................. 17

Combined Modeling Approach ....................................................... 18

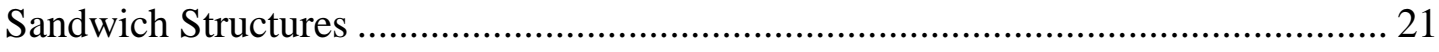

Overview of Work, Process, and Impetus Summary ........................................... 25

3. COUPON DEVELOPMENT AND TESTING .................................................. 28

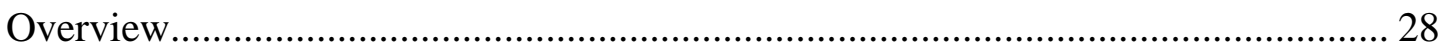

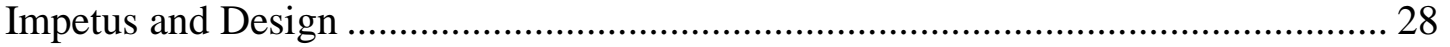

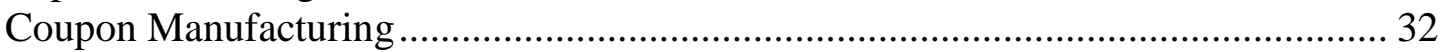

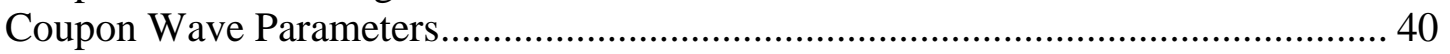

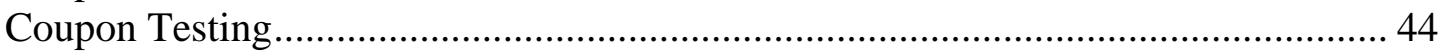

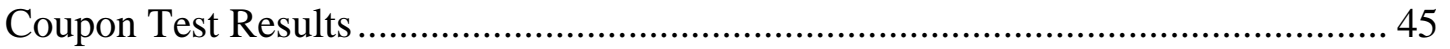

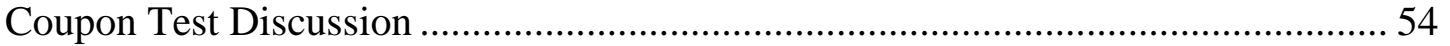

Progressive Failure Mechanics........................................................... 54

DIC Shear Bands ............................................................................. 56

CFIP and Control Comparison .......................................................... 61

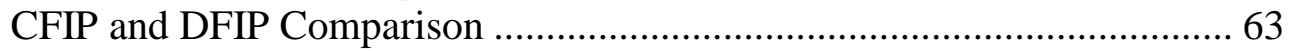

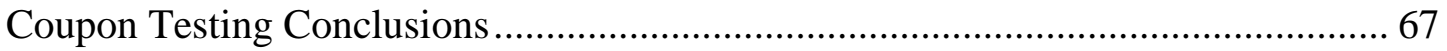

4. COUPON MODELING ............................................................................. 71

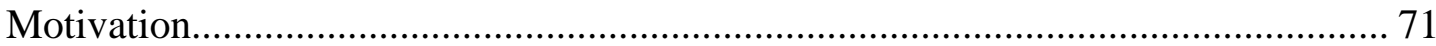

Combined Code Detailed Background and Updates ........................................... 71

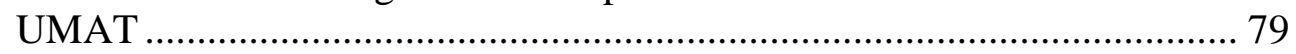

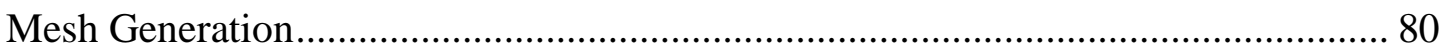

Cohesive Element Material Property Changes …............................................ 82

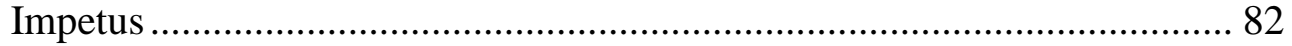

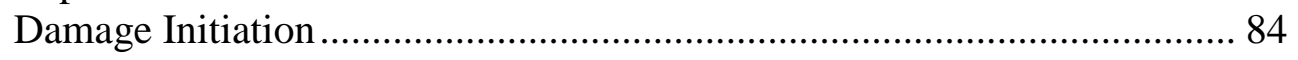


TABLE OF CONTENTS - CONTINUED

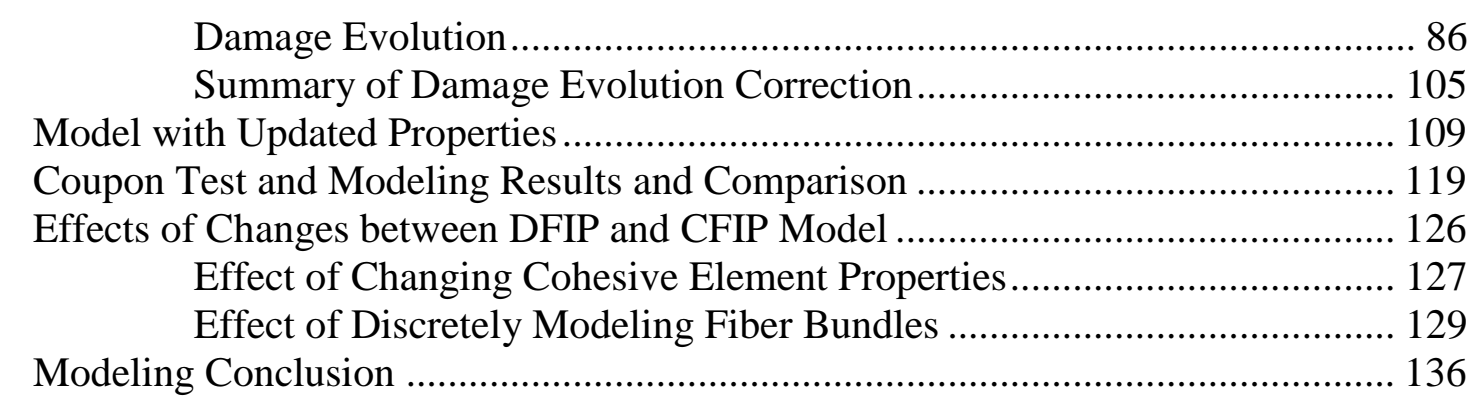

5. SANDWICH BEAM DEVELOPMENT ........................................................ 138

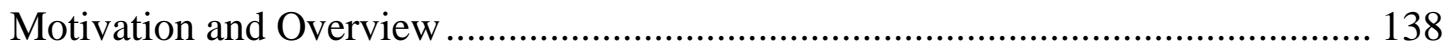

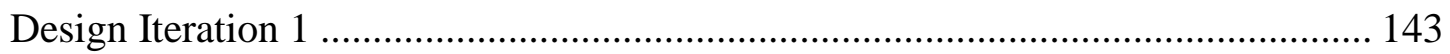

Beam 1 Results .......................................................................... 147

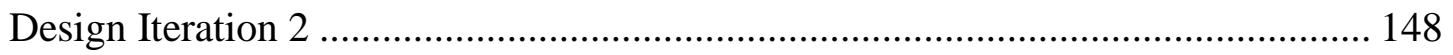

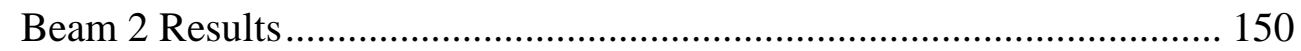

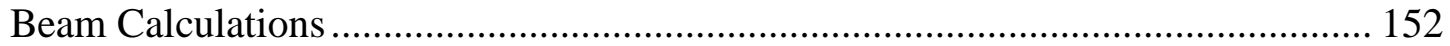

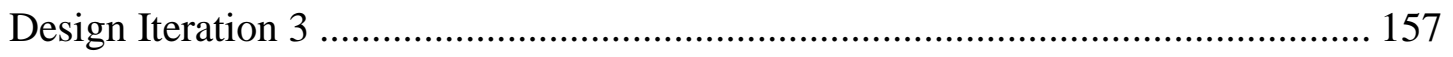

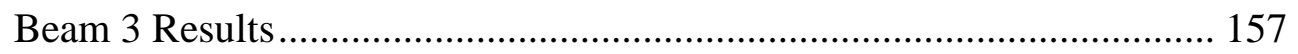

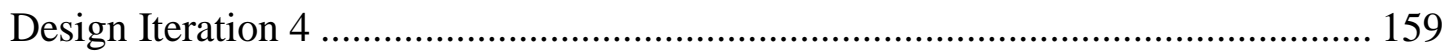

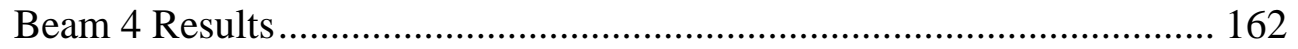

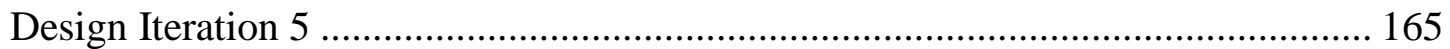

Beam 5 Results .......................................................................... 167

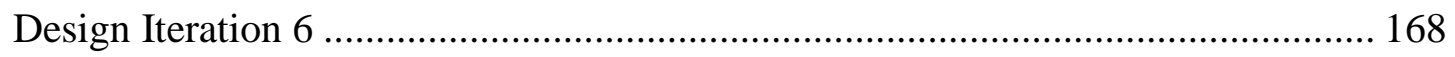

Beam 6 Results ...................................................................... 169

Compressive Flawed Face Sheet Investigation ............................................. 171

Sandwich Beam Discussion and Summary ....................................................... 174

Control Beams ............................................................................... 174

In-Plane Wave Beam.................................................................. 176

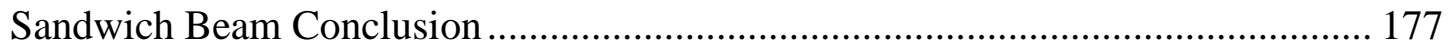

6. CONCLUSIONS AND RECOMMENDED FUTURE WORK ............................ 180

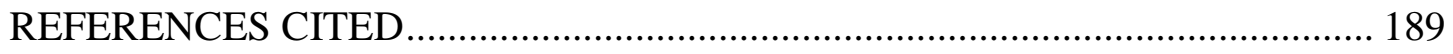

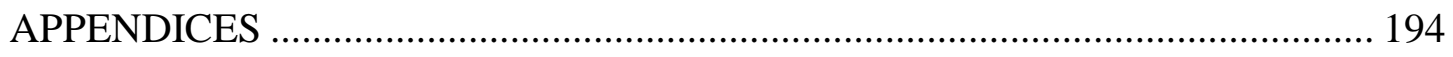

APPENDIX A: Abaqus Input File ............................................................. 195

APPENDIX B: Matlab Code for Abaqus Input File Generation ........................ 202 


\section{LIST OF TABLES}

Table

1. Actions completed in this work and impetus for them. 26

2. Static properties for control and CFIP wave coupons with standard deviation where available and percent of control for comparison.

3. Static properties for DFIP and CFIP wave coupons. DFIP values for wave with misalignment angle around 29 degrees. CFIP values for waves with misalignment angles between 4 and 10 degrees.

4. Material properties from previous testing (Nelson, 2013).

5. Shear stress and strain values from testing to define nonlinear shear response (Nelson, 2013). 76

6. Cohesive element material properties used in previous iteration of model for DFIP wave coupon.

7. Summary of parameters used in the following discussion and calculations for flush, step, and effective flush configurations.

8. Summary of experimental and calculated values for flush, step, and effective flush configurations from above process to determine effective critical energy release rates. 106

9. Sandwich beam ultimate stress and strain at failure values.

10. Comparison of average compressive ultimate stress and strain at failure values for sandwich beams and coupons, both with similar DFIP wave.

11. Sandwich beam face sheet and loading geometry and test results. 177 


\section{LIST OF FIGURES}

Figure $\quad$ Page

1. Typical wind turbine blades (left) and blade failure (right)

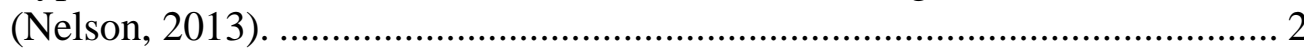

2. Building block test validation pyramid moving from many simple tests to few complex tests (MIL-HDBK-17-1F) ...................................... 5

3. OP wave as seen in skin of wind turbine blade (Riddle, 2013).................... 9

4. IP wave on surface of skin of a wind turbine blade (Riddle, 2013).

5. Explanation of IP wave characterizing parameters for horizontal longitudinal fibers (Riddle, 2013).................................................... 11

6. Fiber tow schematic for discontinuous fiber IP (DFIP) wave coupon. Discontinuous fibers on edges circled.

7. Discontinuous fiber IP (DFIP) wave coupon tested in tension showing discontinuous edge fibers failing.

8. Failure Stress vs. Average Misalignment Angle for discontinuous fiber IP (DFIP) wave coupons (Riddle, 2013).

9. Shear stress-strain response of unflawed specimen used for nonlinear shear UMAT. Tabulated data points shown used for UMAT inputs. (Nelson, 2013).

10. Bi-linear traction-separation criteria defining cohesive element response.

11. Schematic of typical sandwich structure with two face sheets bonded to either side of a core material. (CompositesWorld, 2014)

12. Balsa on bottom and various foam core materials above. (Reinforced Plastics, 2014).

13. Wrinkle defect example in carbon fiber laminate (Hayman, Berggreen, \& Pettersson, 2005). 


\section{LIST OF FIGURES - CONTINUED}

Figure

14. Middle portion of continuous fiber IP (CFIP) wave coupon held up to light. Marks on either side indicate the location down the length of maximum wave amplitude.

15. Schematics of fiber tows in coupons of both wave types, continuous fiber IP (CFIP) wave coupon (left) and discontinuous fiber IP (DFIP) wave coupon (right). Main difference of continuous and discontinuous fibers can be seen by comparing the edges within each wave section shown with the red ovals.

16. Preparing mold to inject resin to make laminate using vacuum resin infusion process. Laying materials on mold (left) and finished lay-up under vacuum (Nelson, 2013).

17. Glass fabric laid over tube to form OP wave with steel bars on either side.

18. Wave form for continuous fiber IP (CFIP) waves as designed in SolidWorks.

19. Wave mold as made (left) and modified by increasing height to increase wave amplitude (right).

20. Table top with removable section for continuous fiber IP (CFIP) wave introduction.

21. Table top middle strip. Removable middle strip (top left), spacer being removed (top right), middle strip dropped down after the spacers have been removed (bottom).

22. continuous fiber IP (CFIP) wave introduction. Wave form in place (top left), glass mat over wave form with steel bars (top right), wave form removal (bottom left), OP wave rotating into IP wave using rounded block (bottom right). Arrows indicate order of steps.

23. CT scan of tool side ply of typical continuous fiber IP (CFIP) wave coupon (left). CT scan averaged over whole thickness of typical continuous fiber IP (CFIP) wave coupon (right). 


\section{LIST OF FIGURES - CONTINUED}

Figure

24. CT scans of cross-sections of continuous fiber IP (CFIP) wave coupon both through the wave portion (left) and outside the wave (right). Thickness variation in the wave can be seen with thickness dimensions labeled at maximum and minimum (left) and a constant thickness can be seen down the length, outside of the wave (right).

25. Test setup from first set of tests (left) and second set of tests (right). The Aramis cameras oriented horizontally for the first set and vertically for the second set.

26. Typical speckle pattern painted on coupon for Aramis readings.

Tabs can be seen on either end of coupon.

27. Images of a CFIP wave coupon during testing from (a) to (f).

Grey lines overlaying coupon show fiber curvature and the arrow indicates the middle of the wave.

28. Typical strain distributions in CFIP wave coupon just before failure as captured with DIC. Longitudinal, transverse, and shear strains are top left, top right, and bottom respectively. Black lines outline the fiber tows in specific locations to show the location of the wave which is located slightly below the middle of the coupon.

29. Coupon schematics from Figure 15 above, showing lengths over which strain is calculated. Strain over $45 \mathrm{~mm}$ wave for CFIP (left) and strain over whole $100 \mathrm{~mm}$ coupon length for DFIP (right).

30. Stress-strain curves for CFIP wave coupons.

31. DIC image of a CFIP coupon showing horizontal displacement just before failure. Black lines outline the fiber tows in specific locations to show the location of the wave which is located slightly below the middle of the coupon.

32. Strain regions for calculating half-wave symmetry shown on schematic of wave region. 


\section{LIST OF FIGURES - CONTINUED}

Figure $\quad$ Page

33. DIC shear strain distribution in control coupon. Pattern of alternating positive and negative shear band spacing labeled as 1

$\mathrm{cm}$.

34. Glass mat showing angled stitching separating fiber bundles. Scale is in centimeters. Pattern is repeated down the length every $1 \mathrm{~cm}$. 58

35. CFIP wave and control coupons shear strain fields. Shear band spacing can be seen to be the same between the two coupons.

36. Typical stress-strain curves for control and CFIP wave coupons.

37. Typical stress-strain curves for control and discontinuous fiber IP (DFIP) wave coupons tested in tension (Nelson, 2013), (Riddle, 2013).

38. Failure Stress vs. Misalignment Angle for DFIP data and CFIP data. DFIP data as discrete points and CFIP data over range of misalignment angles.

39. Glass fiber mat showing $4 \mathrm{~mm}$ width fiber bundles separated by stitching......

40. Experimental longitudinal strain field obtained with the DIC showing high strain regions separated by fiber bundle distance of $4 \mathrm{~mm}$.

41. CFIP wave coupon model mesh. Cohesive columns can be seen as darker columns spaced every 8 continuum elements across the width.

42. Traction-Separation curve for bi-linear criteria defining cohesive element damage.

43. Picture looking down length of coupon with width and thickness directions labeled. Complex cross-section geometry of fiber bundles as well as resin rich location separation can be seen. 50 $\mathrm{mm}$ coupon width and $4 \mathrm{~mm}$ fiber bundle width labeled. 


\section{LIST OF FIGURES - CONTINUED}

Figure $\quad$ Page

44. Schematic cross-sections of coupon geometry 2 fiber bundle widths wide and all 4 plies thick. Flush configuration with perfectly aligned bundles between plies as assumed by the model (left). Step configuration taking into account through-thickness complexity seen in actual coupon geometries (right). 88

45. Schematic cross-sections of 2 fiber bundles wide and all 4 plies thick. Flush configuration (left) and step configuration (right). Crack necessary for through-thickness resin failure shown by line.

46. Models for calculating energy for crack propagation in Mode I for both flush configuration (top) and step configuration (bottom). Crack shown in red. Arrows depict global Mode I loading direction.

47. Models for calculating energy for crack propagation in Mode I for both flush configuration (top) and step configuration (bottom) after cohesive elements have failed.

48. Load-Displacement curve for step configuration model in Mode I loading.

49. Load-Displacement curves for step and flush configuration models. Step configuration with original $G c$ values and flush configuration with maximum effective $G c$ values.

50. Load-Displacement curves for flush and step configurations, both with experimental $G c$ values, demonstrating difference in energy to failure (area under curves).

51. Load-Displacement curves for flush configuration with experimental values and updated values.

52. Stress vs. Strain plot for CFIP wave coupon model up to $2.56 \%$ strain. Failure and associated truncation has not been applied.

53. Stress vs. Strain plot for CFIP wave coupon model after failure criteria has been applied. Failure would occur somewhere on the dashed line. 


\section{LIST OF FIGURES - CONTINUED}

Figure

54. Longitudinal strain field at 1.17 (a), 2.37 (b), and 2.45 (c) $\%$ longitudinal strain over the wave, showing damage progression. Strain values correspond to first cohesive element damage (a), ultimate stress (b), and final failure (c).

55. Single fiber bundle longitudinal strain distribution showing four regions of high strain near locations of maximum curvature. Coupon included showing location of fiber bundle investigated.

56. Transverse strain field at 1.17 (a), 2.37 (b), and 2.45 (c) $\%$ longitudinal strain over the wave, showing damage progression. Strain values correspond to first cohesive element damage (a), ultimate stress (b), and final failure (c).

57. Shear strain field at 1.17 (a), 2.37 (b), and 2.45 (c) \% longitudinal strain over the wave, showing damage progression. Strain values correspond to first cohesive element damage (a), ultimate stress (b), and final failure (c).

58. Cohesive element damage at 1.17 (a), 2.37 (b), and 2.45 (c) \% longitudinal strain over the wave, showing damage progression. Strain values correspond to first cohesive element damage (a), ultimate stress (b), and final failure (c). Blue elements are undamaged and red elements are damaged.

59. Analytical and Experimental Stress vs. Strain curves showing good analytical-experimental correlation.

60. Longitudinal strain field near ultimate stress experimental (left) and analytical (right). Arrow indicates maximum wave amplitude location down length.

61. Transverse strain field near ultimate stress experimental (left) and analytical (right).

62. Shear strain field near ultimate stress experimental (left) and analytical (right).

63. Stress vs. Strain curves for model with original as well as adjusted cohesive element material properties. A typical experimental curve is included for comparison. 


\section{LIST OF FIGURES - CONTINUED}

Figure $\quad$ Page

64. Analytical Stress vs. Strain curves for $1 \mathrm{~mm}$ and $4 \mathrm{~mm}$ cohesive element spacings with original cohesive element properties showing no difference in response. Experimental curve included for comparison.

65. Analytical Stress vs. Strain curves for $1 \mathrm{~mm}$ and $4 \mathrm{~mm}$ cohesive element spacing with adjusted cohesive element properties. Experimental curve is included for comparison.

66. Longitudinal strain field over wave section of model with $1 \mathrm{~mm}$ cohesive element spacing with adjusted cohesive element properties. Spacing between high strain regions can be seen and labeled as $4 \mathrm{~mm}$ spacing despite cohesive element spacing.

67. Subscale test frame with actuator and load rollers.

68. Four-point bending diagram showing shear stress and moment distribution along the length (what-when-how, 2014).

69. Segmented balsa (top) and sheet balsa (bottom) with 1 foot ruler for scale.

70. C-channel mold (left) and C-channel composite beam made on mold.

71. Sandwich Beam 1 looking down the length to show cross-section.

72. Beam 1 tension side face sheet (top) and compression side face sheet (bottom) showing load pad locations and strain gage configurations circled in red.

73. Beam 1 loaded in test frame.

74. Strain vs. Load curves for compressive and tensile face sheets for Beam 1. 148

75. Beam 2 cross-section. 149

76. Half of Beam 2 during testing. 150 


\section{LIST OF FIGURES - CONTINUED}

Figure $\quad$ Page

77. Strain vs. Load curves for compressive and tensile face sheets for Beam 2.

78. Beam 2 balsa shear failure and delamination.

79. Beam 2 schematic cross-section labeled for calculations.

80. Strain vs. Load curves for compressive and tensile face sheets for Beam 3. 158

81. Beam 4 on mold and under vacuum ready for injection. Injection on close end and vacuum on far end.

82. Thermocouple and controller on mold just before injection.

83. All three thermocouples on beam and mold during injection. 1 controller thermocouple on mold and 2 logging thermocouples....

84. Beam 4 in test frame before applying load.

85. Beam 4 loaded and at maximum actuator displacement before adjusting to take advantage of full actuator travel.

86. Strain vs. Load curves for compressive and tensile face sheets for Beam 4.

87. Beam 4 loaded and near maximum actuator travel reached by using wooden spacers shown.

88. Beam 5 with middle injection and end vacuum ports.............................. 166

89. Beam 5 compressive face sheet failure.

90. Strain vs. Load curves for compressive and tensile face sheets for Beam 5.

91. 6.5 inch $(17 \mathrm{~cm})$ wide glass mat to be compressive side face sheet showing DFIP wave.... 


\section{xiv \\ LIST OF FIGURES - CONTINUED}

Figure $\quad$ Page

92. Beam 6 compressive face sheet with strain gage array and failure. Wave extends across the width with maximum amplitude along the middle. Failure occurs near maximum misalignment angle and extends across the width.

93. Strain vs. Load curves for compressive and tensile face sheets for Beam 6. Compressive face sheet strain gage locations as shown in Figure 92

94. Stress vs. Strain Magnitude for far-field, flawed compressive face sheets for sandwich beams 6 through 8 . 


\begin{abstract}
Defects in materials can reduce strengths and lifetimes of manufactured parts. The number of possible defects increase with the complexity inherent in composite materials. The wind industry uses composite wind turbine blades in which the manufacturing process induces a number of defects. In order for the wind industry to continue sustainable expansion, the effects of defects must be better understood.

In-plane (IP) fiber waviness is the focus of this work. The three main parts of this work include testing on the coupon level, modeling on the coupon level, and testing of beams in four-point bending (with and without defects). The coupon level testing includes partial IP waves, similar to those in manufactured parts, rather than full width IP waves. This allows investigation into complex interactions and varying failure mechanisms caused by the fiber misalignment gradient. Partial waves are also modeled to both validate testing as well as to increase robustness of a previously developed progressive damage modeling method. Lastly, a sandwich beam test specimen for testing in 4-point bending is developed to investigate the effects of fiber waviness in both tension and compression when loaded in flexure.
\end{abstract}




\section{CHAPTER 1}

\section{INTRODUCTION}

\section{$\underline{\text { Motivation }}$}

Composite materials are becoming more commonly used due to their large strength to weight ratios and customizable material properties due to anisotropy. Additional benefits are fatigue and corrosion resistance. The material group called "composites" is a very broad term that is not reserved for human-made materials. Wood and cartilage are two examples of naturally occurring composites. Composites are defined as a material made of two or more materials that, when combined, form a material with enhanced properties (Barbero, 2011). Typically, one constitutive material carries the majority of the load while the other material is used to hold the shape and distribute the load into the strong reinforcing material. The load carrying material is the reinforcement and the load distributing material is the matrix. Common reinforcement materials are continuous fibers, chopped fibers, or aggregate of small pieces. The fibers are often made of glass or carbon and the aggregate is often rock, as used in concrete. Fiber composites have many applications ranging from small parts, such as bicycle seat posts and derailleurs up to bicycle frames, car panels, boat hulls, airplane panels, and wind turbine blades.

The work herein focuses on composites commonly utilized by the wind turbine blade industry. Typical wind turbines are shown in Figure 1 (left) along with a blade failure (right). Wind energy is the leading renewable energy source for installed power 
generation capacity. This industry has been growing rapidly over the past fifteen years and is expected to continue its growth (AWEA, 2013). For this growth to continue, certain structural obstacles must be overcome.

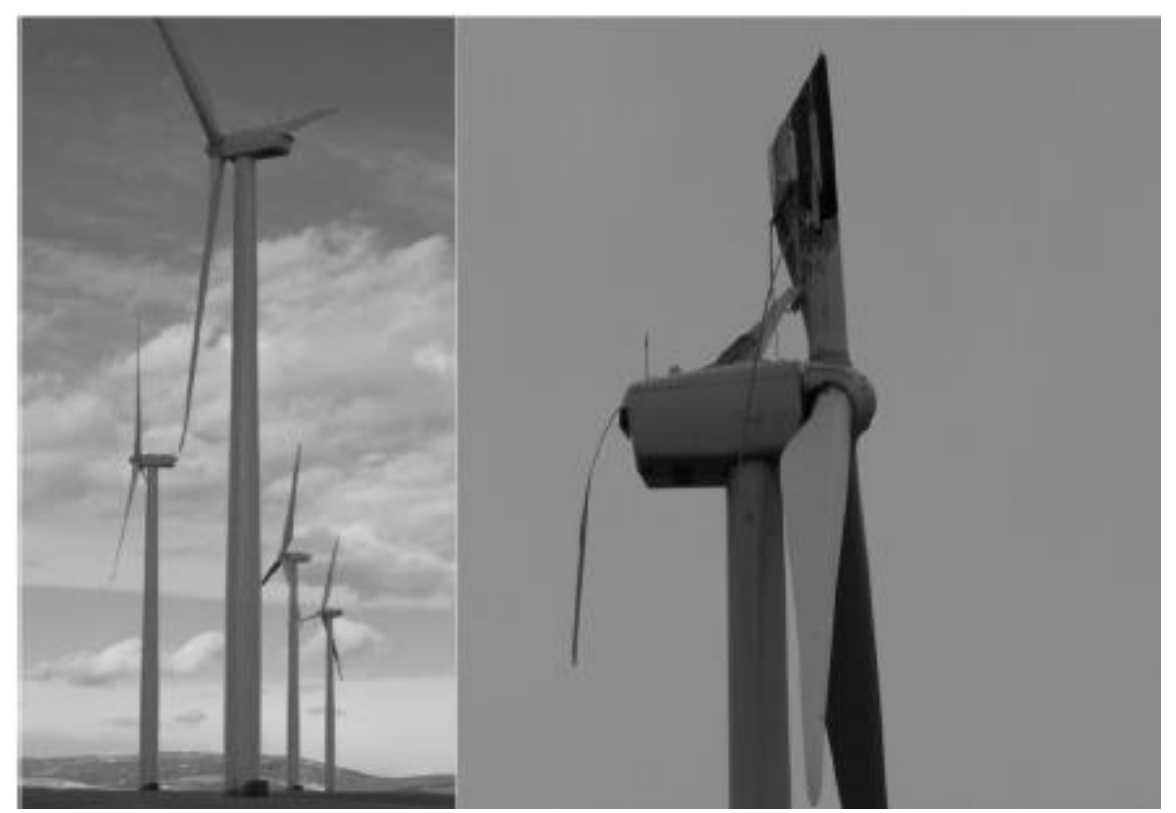

Figure 1: Typical wind turbine blades (left) and blade failure (right) (Nelson, 2013).

The United States has taken an interest in renewable energies. Wind energy is the fastest growing renewable energy based on new power generation capacity (AWEA, 2013). The United States has passed a policy which set a target of $20 \%$ of the country's electricity supply to come from wind power by 2030 (Department of Energy, July 2008). Due to the country's interest in wind energy, the U.S. Department of Energy started the Blade Reliability Collaborative (BRC). The goal of this collaborative is to improve reliability in order to achieve target lifetimes of 20 years (Paquette, 2012). Not just will more wind turbines increase wind energy power production, but also larger turbines will increase production of each turbine. The power production potential for a turbine is 
limited by the amount of energy passing the blades in the form of wind. Increasing the swept area by the blades increases this area and as blade length increases, swept area increases exponentially. For this reason, there is a push to make larger wind turbine blades which increases the need to fully understand the failure mechanisms in these blades.

One of the obstacles faced by wind energy is the costly loss of energy production potential due to downtime of turbines caused by preventative maintenance or repairs (Paquette, 2012). A 2008 study by Sandia National Laboratory found that $7 \%$ of wind turbine blades had to be replaced before reaching their expected lifetime. Due to each turbine having three blades, the chance of downtime caused by a blade failure is tripled (Cairns, Riddle, \& Nelson, 2011). According to Sandia, blades have been responsible for $11 \%$ of turbine downtime recently. This number is expected to increase due to the increase in size (Sandia National Laboraties, 2014). An example of this was a Vestas wind turbine blade failure in 2012 which was deemed to have been caused by fiber waviness in the spar cap (Suzlon Blade Recall, 2010). Other blade problems arise from poor bonding and environmental issues such as erosion, freeze/thaw cycling, and lightning. A preliminary operator survey found that replacement times for a blades range from 2 weeks to 2 months (Paquette, 2012).

While wind turbine down time for maintenance is not desired, it is much preferred to a blade failure. Failed blades are dangerous and potentially destructive to the tower and other blades (Gebert, 2012). Failed blades can result in more downtime than a repair and, due to their large-scale, can cause fear and a backlash in public support. One of the 
larger blades is made by Siemens and is $75 \mathrm{~m}$ long, weighing up to 25 tons (Siemens, 2012). These blades are often $80 \mathrm{~m}$ above the ground (Riddle, 2013). For the wind industry to continue growing, the reliability of wind turbines must be increased so that the public does not fear these but, instead, focuses on the clean energy production.

A major problem of designing with composites is a lack of consistency in manufactured parts. This is because composites are much more complex than commonly used isotropic metals such as aluminum and steel. The complexities arise from anisotropy and defects. There are more types of defects in composites due to a more involved manufacturing process. In order for composites to be more widely used they must be better understood (Nelson, 2013). As of now, composite products are often overdesigned because the actual material properties of the final part vary more greatly from the properties used in design calculations. This requires large safety factors. Overdesigning parts as large as wind turbine blade greatly increases the weight and limits the size, reducing the advantages of using composites. This method still results in unknown expected lifetimes. Eliminating defects is not practical which means that the only other option is to understand the effects of the defects. By understanding the effect of a defect, post-manufacturing analysis can be performed to check if safety criteria is met as well as the new expected strength and lifetime.

Guidelines currently exist to determine the allowables for a composite part. These are outlined in the Composites Material Handbook (MIL-HDBK-17-1F). This process is extensive, requiring much testing at different levels, as can be seen in Figure 2. This shows the experimental process required to understand a complex component. 
Complexity of the part increases bottom to top, from coupon level to component level.

The price both monetarily and temporally increases greatly upon moving up the pyramid.

For this reason, the number of tests decreases as complexity increases.

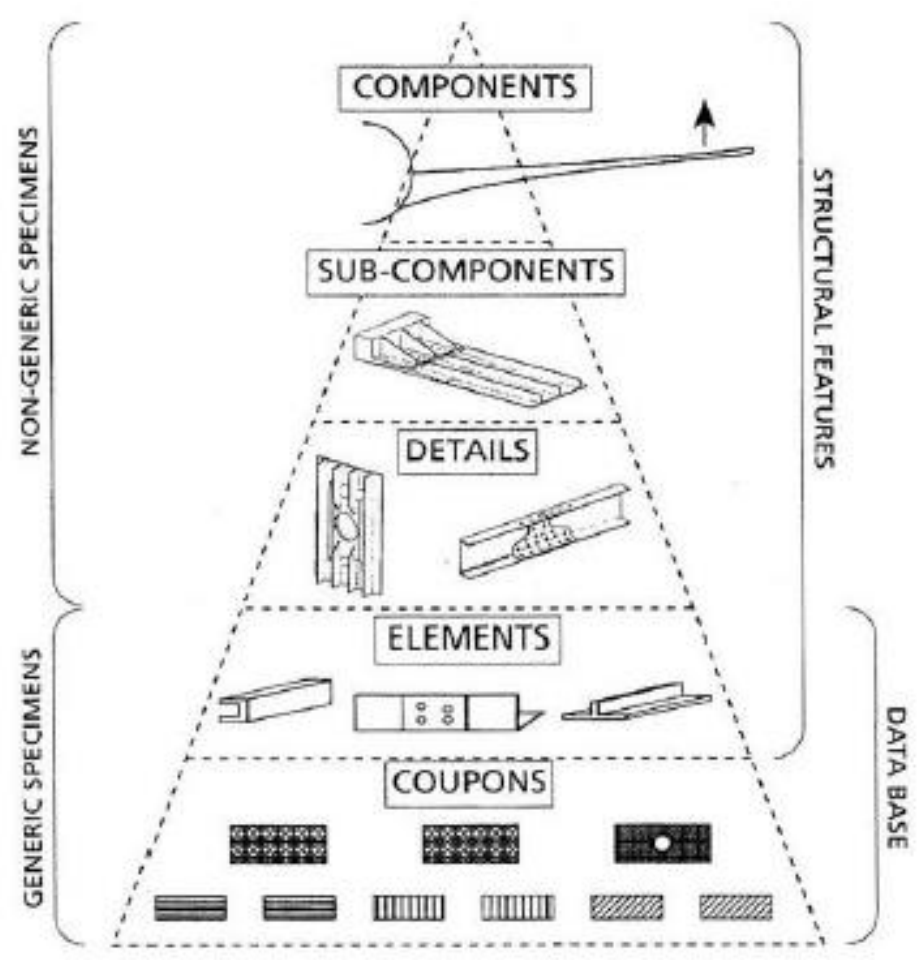

Figure 2: Building block test validation pyramid moving from many simple tests to few complex tests (MIL-HDBK-17-1F).

The Montana State University Composites Group (MSUCG) is tasked with investigating the effects of defects commonly found in wind turbine blades (Sandia National Laboraties, 2014). These are bonding and manufacturing defects such as fiber waviness and porosity. The subtasks within effects of defects are flaw characterization and effects of defects. The scope of this work focuses on effect of defects, expanding on previous work by increasing defect and specimen complexity as suggested as future work by Nelson, 2013. This was accomplished by testing composite coupons with fiber 
waviness, improving robustness and accuracy of MSUCG's previously developed progressive damage modeling method, and develop a composite-balsa sandwich beam for four-point bending to further assess the effects of defects.

The wind turbine blade industry is growing and for rapid, sustainable growth to continue as desired, a better understanding of the effects defects is necessary. Significant initial research has been performed in the field of effects of defects laying the groundwork to build upon. This field is expanded upon herein by increasing the complexity of defect and mechanical testing and by further developing a progressive damage model.

\section{Overview}

As noted above, the work herein focuses on two sides of the Effects of Defects subtask of the BRC. The first side is to investigate the effect of in-plane waves with geometry more similar to those found in manufactured parts. This is accomplished through mechanical testing and modeling of coupons with in-plane waves of varying severity as opposed to previous waves of constant severity. This type of wave will avoid initial failure and load redistribution induced by discontinuous edge fibers. Modeling this in-plane wave will improve the robustness of the previously developed progressive damage model. The second side is developing a composite-balsa core sandwich beam for four-point bend tests. This allows for the effects of defects to be investigated in flexure in order to start bridging the gap between the extensive effects of defects work done on the coupon level and the full-scale wind turbine blade. 


\section{CHAPTER 2}

\section{BACKGROUND}

The work herein can be applied to fiber composites in many different industries but is tailored to the wind turbine blade industry. First, the BRC will be discussed in more detail to give more background and motivation for this research. A brief summary of defects common in wind turbine blades follows. Next, past research of effects of defects is presented, focusing on recent MSUCG work. The MSUCG research includes experimental and analytical work including a combined continuum/discrete modeling method that is further developed herein. This section concludes with a discussion of past research implementing composite face sheets in sandwich structures.

\section{$\underline{\mathrm{BRC}}$}

As mentioned above, the BRC is a DOE funded collaborative with the goal of increasing the reliability of wind turbine blades in order to decrease the frequency of costly downtime due to repairs. This collaborative is made up of wind farm owners and operators, turbine manufacturers, and third party investigators and is led by Sandia National Laboratories. There are six main sections of work included in the BRC (Paquette, 2012):

1. Blade Defect and Damage Database

2. Inspection Validation

3. Effects of Defects

4. Analysis Validation 
5. Certification Testing

6. Standards and Partnerships

The MSUCG is a third party investigator tasked with investigating the Effects of Defects. This has been broken into two subtasks (Cairns, Riddle, \& Nelson, 2011):

1. Flaw characterization

2. Effects of Defects

MSUCG research has already performed research on both of these subtasks as discussed below. The research herein will continue the Effects of Defects research.

\section{$\underline{\text { Common Blade Flaw Types }}$}

As mentioned above, the laminate flaw types common in wind turbine blades are porosity and fiber waviness. Porosity is the inclusion of air pockets inside the laminate which cause material property degradation. Much experimental research has been performed investigating porosity. Also, previous work on modeling porosity has done an adequate job with simple modeling methods (Nelson, 2013), therefore, porosity is not investigated further in this work.

Fiber waviness is the other main type of flaw found in wind turbine blade laminates. Due to fiber orientation being shifted off-axis, the matrix is required to carry more load rather than the strong fibers. This results in a loss of strength. Fiber waviness also decreases compressive strength due to the fibers are starting in a semi-buckled orientation making a buckling failure more likely (Avery, 2004). The wave category of defect is broken into out-of-plane (OP) waves, in which the wavy fibers are elevated out of the plane of the laminate, and in-plane (IP) waves, where the fibers remain in the 
plane. An OP wave is shown in Figure 3 in a cross-section of the skin of a wind turbine blade. An IP wave, also in the skin of a blade, is shown in Figure 4. OP waves have a large effect on compressive strength, decreasing compressive strength by up to $36 \%$ (Adams \& Bell, 1995), (Adams \& Hyer, 1993). Previous OP wave coupons used for research have been deemed applicable and sufficient so that they will not be investigated further herein.

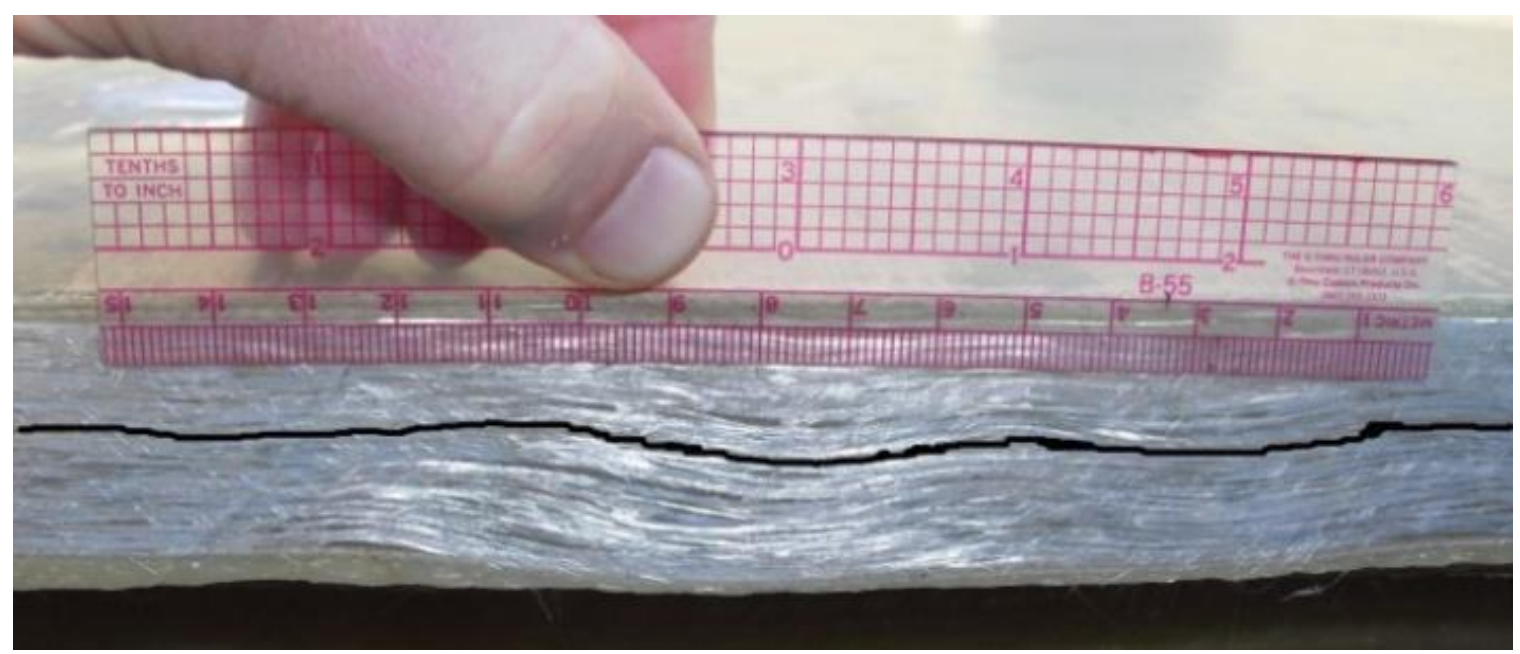

Figure 3: OP wave as seen in skin of wind turbine blade (Riddle, 2013).

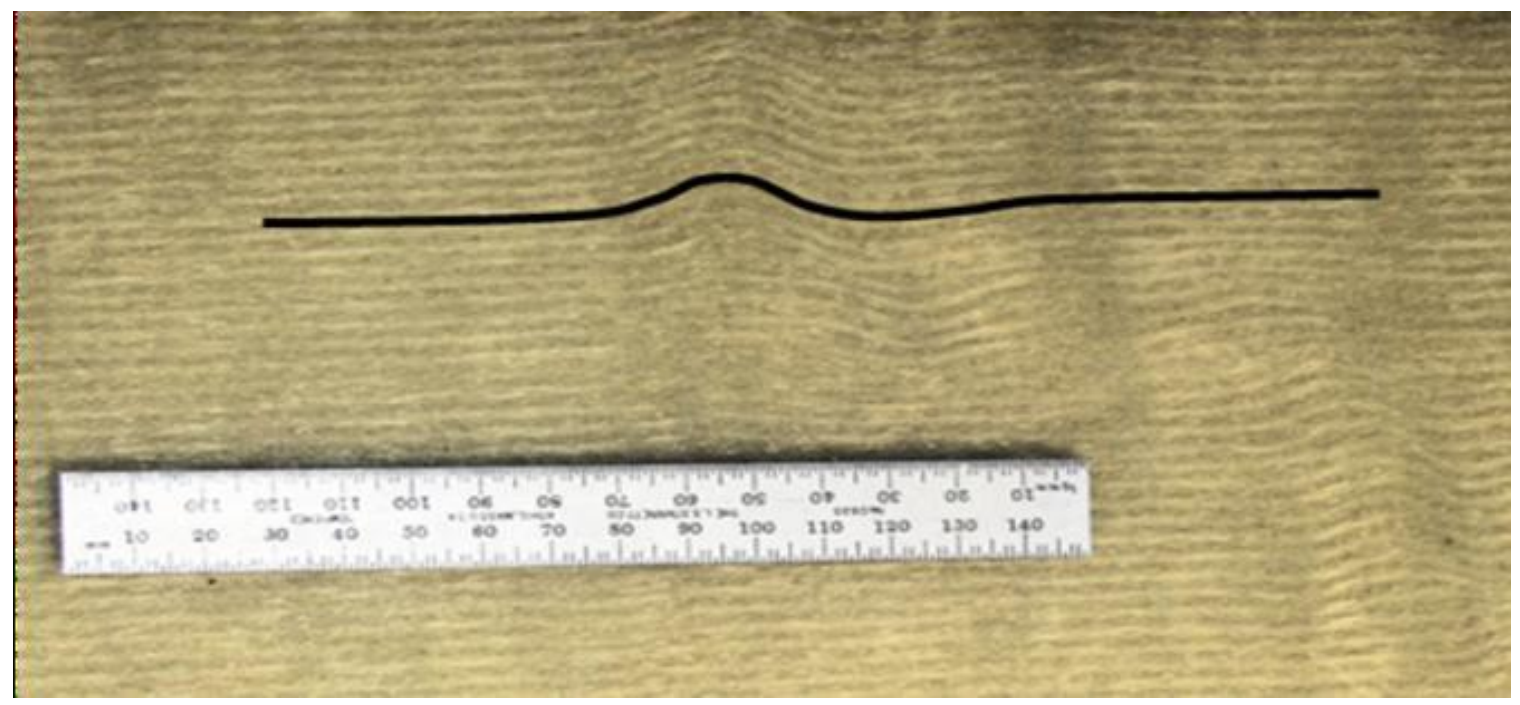

Figure 4: IP wave on surface of skin of a wind turbine blade (Riddle, 2013). 
Many wind turbine blades are made using vacuum resin infusion. This manufacturing method makes IP waves more likely to occur (Wang, 2012).

\section{$\underline{\text { Past MSUCG Experimental Research }}$}

Previous research has been performed on quantifying and characterizing the effects of defects. This work has included performing survey work on waves in manufactured blades, determining the critical flaw parameter in waves through experimental testing, and developing a predictive progressive damage model for waves in laminates. The blade defect survey and experimental portions of this work are discussed in this section.

Parameters for characterizing IP waves were previously determined to be amplitude, wavelength, and fiber misalignment angle. These can be seen in Figure 5. The amplitude is the perpendicular distance from the unperturbed fiber tow to the point in the wave farthest from this in the same tow. Wavelength is the length over which the wave extends in the direction of unperturbed fibers. Misalignment angle is the maximum angle that the tow makes in the wave measured against the unperturbed fiber direction. The angle of each fiber tow within the wave varies down the length. The maximum fiber misalignment angle is the largest angle reached as can be seen in Figure 5 at one-fourth and three-fourths wavelength. 


\section{Characterization of Wave Segment}

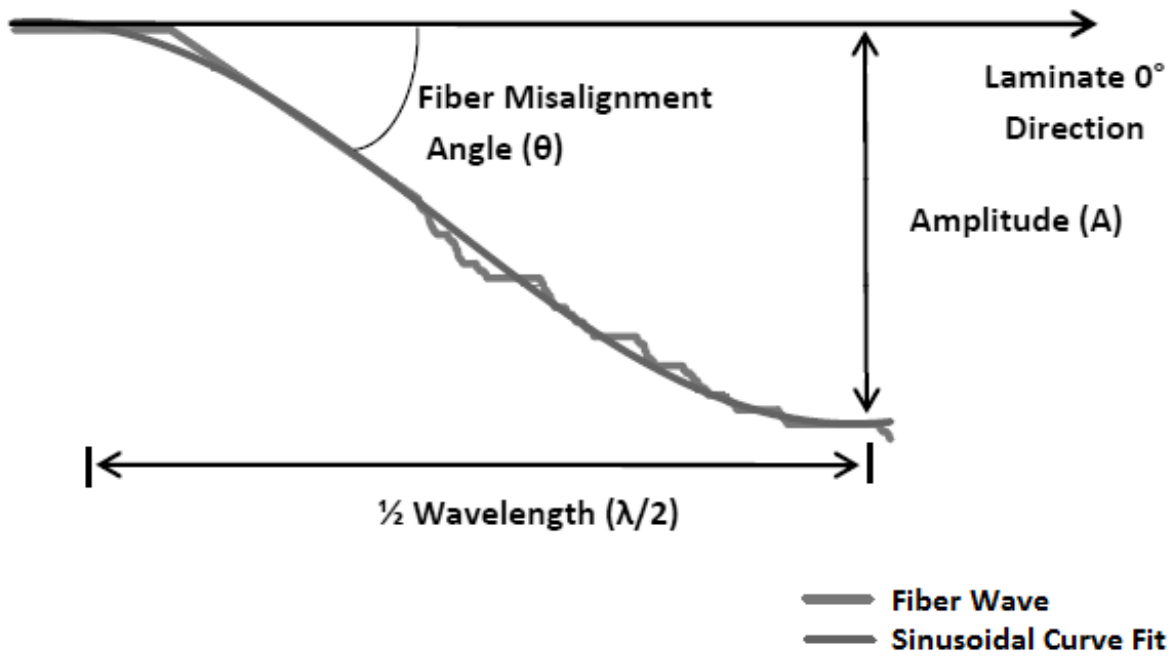

Figure 5: Explanation of IP wave characterizing parameters for horizontal longitudinal fibers (Riddle, 2013).

Before wave coupons were made and tested, a blade survey was performed in which 5 blades were investigated and wave parameters, locations, and prevalence were recorded. This included 4 blades at NREL's National Wind Technology Center which ranged from 1 to $2.5 \mathrm{MW}$ size and 1 blade at Sandia National Laboratory. IP wave misalignment angles were recorded and a range of 8 to 52 degrees was found, with a mean of 28 degrees (Riddle, 2013).

The experimental process for the effects of defects research started with a large number of axial loading coupon tests. These were performed on both carbon and glass fiber coupons and on varying layups in compression and tension. The glass coupons were made of 4 unidirectional glass plies, [(0) 4 , of PPG-Devold fiberglass with a Hexion resin and hardener, manufactured using the vacuum resin infusion process. 
The manufacturing processes for both control and IP coupons are described in detail in Nelson (2013) and Riddle (2013). Due to this process being similar to the manufacturing process used for the work herein, this is also discussed in more detail in Chapter 3. The IP waves were introduced by laying each layer of glass over a tube causing an OP wave. The tube was removed and the $\mathrm{OP}$ wave was pushed and dragged into the plane to create an IP wave.

Cutting coupons from this wave laminate resulted in discontinuous fibers on either side due to the wave extending through the whole laminate. This can be seen in the fiber tow schematic in Figure 6. The tension coupons were $50 \mathrm{~mm}$ wide and $200 \mathrm{~mm}$ long. After tabs were glued to the coupons, the gage length was $100 \mathrm{~mm}$.

Due to slight variation between the maximum fiber misalignment angle between plies, it was determined that the average misalignment angle over all plies was to be used as the fiber misalignment parameter (Riddle, 2013). The misalignment angles in each ply were investigated through the use of CT scans to view individual plies in prepared coupons. 


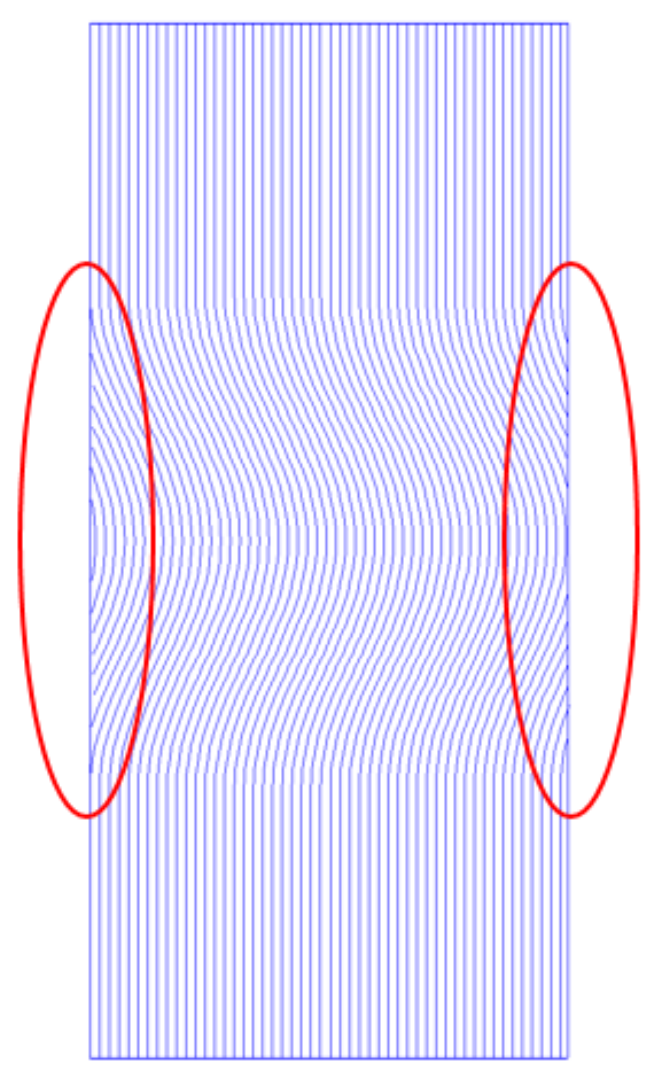

Figure 6: Fiber tow schematic for discontinuous fiber IP (DFIP) wave coupon. Discontinuous fibers on edges circled.

These coupons were tested in tension and compression. Due to the work herein focusing on tension due to testing difficulties inherent with compression, discussion of previous research focuses on tensile tests. The results of these tests are included in Chapter 3 for experimental comparison with the more complex wave coupons developed and tested herein.

During tensile testing, the discontinuous fibers along the edges, as seen in Figure 6, popped off during testing. This occurred near $0.5 \%$ strain and can be seen in Figure 7. This coupon is called the discontinuous fiber in-plane (DFIP) wave coupon. 


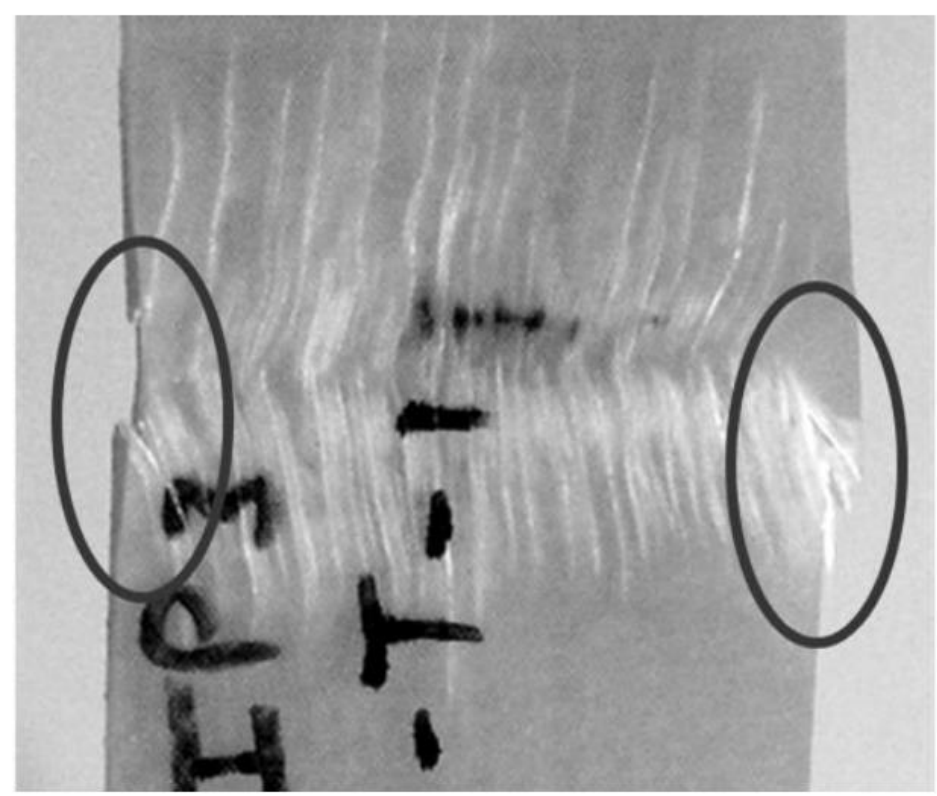

Figure 7: Discontinuous fiber IP (DFIP) wave coupon tested in tension showing discontinuous edge fibers failing.

A multitude of wave coupons with varying wave parameters were tested. Figure 8 shows the failure stress plotted against the average misalignment angle in both tension and compression.

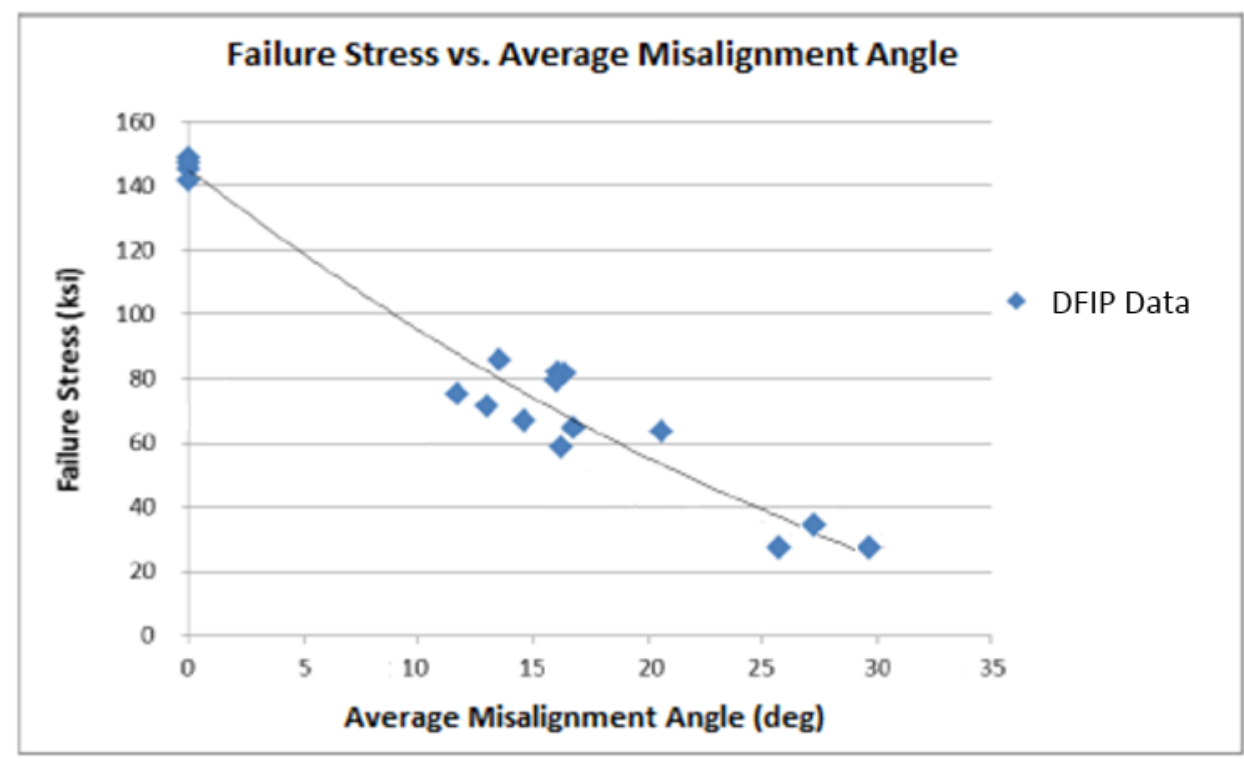

Figure 8: Failure Stress vs. Average Misalignment Angle for discontinuous fiber IP (DFIP) wave coupons (Riddle, 2013). 
The critical flaw parameter is the wave parameter which determines the degradation caused by the presence of the wave. It was found that the average misalignment angle is the critical flaw parameter for these waves. This was further supported by finding that waves with different amplitudes and wavelengths but the same misalignment angle have similar results (Nelson, 2013).

It should be noted that the discontinuous fibers in this wave type are not typical of waves found in manufactured parts, as seen in Figure 4. The response was largely influenced by these discontinuous fibers which means the response of these DFIP wave coupons may not be typical of the response of waves in manufactured parts.

\section{Composite Modeling Approaches}

Finite Element Modeling (FEM) is a very powerful tool for calculating the response of materials of complex geometries under various loading conditions. FEM has many uses for many materials and in many industries. One of these uses is to model composite materials within the domain of mechanical loading. Modeling a material response gives insight into the mechanics of a problem and can provide validation to a test method. A model can also be predictive which allows for the response of untested material geometries and loading conditions to be known without testing. A predictive progressive damage model has been a goal of the MSUCG.

Understanding and modeling damage progression first requires a definition of damage. While many definitions exist, damage here is considered any unrecoverable loss of energy in the composite. This can be the creation and progression of a crack. 
A common basis for modeling is micromechanics. This however is limited to simple, unflawed geometries, primarily unflawed flat plates and beams (Kassapoglou, 2013). Two more robust methods are Continuum Damage Modeling (CDM) and Discrete Damage Modeling (DDM), which are discussed in detail below.

Along with the type of modeling method, the failure criteria must also be chosen. Failure criteria are used to determine when failure has occurred. Some of these are maximum stress, maximum strain, Tsai-Hill, Tsai-Wu, and Hashin (Barbero, 2011). Due to none of these failure criteria being used, for reasons explained below, details on these failure criteria are omitted here.

The typical flow of a progressive damage model as discussed so far is as follows. The inputs include a mesh, material definitions and properties, boundary conditions, loads, and solver and output definitions. The model then runs the first iteration of calculations and checks for failure based on the failure criteria chosen. If no failure has occurred then the next iteration of calculations occurs. This continues until the first failure has been reached. At this point, damage is applied to the local failure. The model may then check if the damage caused by this local failure has caused ultimate failure or not. If not, then the model continues to the next iteration. When ultimate failure has occurred, the model will stop. Determining ultimate failure is difficult and is often considered to be based on maximum attained load or to be at first fiber failure or first ply failure. The way in which damage is accounted for depends largely on the modeling approach used. CDM and DDM have very different methods. 


\section{Continuum Damage Modeling}

CDM looks at the entire laminate as one material which requires the individual material properties of the fiber and matrix to be combined and smeared over the whole laminate. Due to this smearing, fiber-matrix interactions are lost. Failure criteria is therefore based on global material properties. This method is based on an approach developed by Kachanov $(1958,1986)$ which uses the increasing entropy caused by damage to quantify the damage to change material structure (Nelson, 2013). There are different ways of implementing the CDM approach. The simple approach is material property degradation method. Using this method, some or all of the elastic properties $\left(E_{1}, E_{2}, v_{12}, v_{23}, G_{12}\right)$ are reduced by a constant once damage has initiated. The properties are then set to zero once the element has reached final failure. Choosing this damage value is difficult and each property's degradation factor may need to be tuned

individually to get good correlation (Camanho \& Matthews, 1999). Due to the necessity of tuning for correlation, predictive capabilities are limited.

CDM is a simple method which is not computationally expensive. However, this method alone is not sufficient for an accurate progressive damage model. There are different methods of implementing the CDM approaches but overall they lack predictivity and including flaws is difficult.

\section{Discrete Damage Modeling}

The DDM approach models both the fiber and matrix discretely as separate element types with separate material properties. This allows local failures to be modeled as they occur. The resin is modeled with cohesive elements while the fibers are modeled 
with continuum elements. Cohesive elements are used to model interfaces which makes them a good candidate for modeling resin between fiber tows. These can be governed by a traction-separation law (Abaqus Analysis User's Manual v6.12, 2012) which is wellsuited for modeling resin. A large advantage of the DDM approach is that local failures, such as matrix cracking, are captured explicitly by cohesive element degradations and failures. This is typically considered a more accurate modeling method than CDM. Accounting for damage, DDM includes local failures whereas CDM changes the bulk material properties to try to account for the global effect of local damage. The disadvantages of DDM are larger computational expense and more time required for meshing as well as the results being more mesh dependent (Nelson, 2013).

\section{Combined Modeling Approach}

A comprehensive study was undertaken by MSUCG to determine the best way of modeling fiber waviness in the coupons discussed above (Nelson, 2013). Both IP and OP waves were investigated in both glass and carbon. The laminates included unidirectional and +/- 45 layups. Accuracy of the model was determined based on correlation between model and testing. This was performed qualitatively, with visual investigation of failure progression, as well as quantitatively, by comparing initial stiffness and overall stressstrain response.

The iterations of model types started simple and increased in complexity trying to achieve better experimental correlation and predictive ability. All models were 2D, starting with simple CDM approaches and expanding to DDM approaches. The final method included a combination of both CDM and DDM methods. Fiber tows were 
modeled discretely by using the DDM method to separate fiber tows with cohesive elements, representing resin richness. The fiber tows were modeled using the CDM method. Looking at Figure 6 above, the blue lines represent the cohesive elements which model the resin which separates the fiber tows.

A nonlinearity was observed in the shear response in the matrix. A user material card (UMAT) was used in attempt to capture this non-linear shear softening behavior. Accurate shear response is important for good correlation because shear between fiber tows is the cause of initial damage. The UMAT updates the shear strain based on the shear stress-strain curve. This curve was created based on the ASTM D3518 in-plane shear response test and can be seen in Figure 9 (ASTM D3518). While there is a softening as seen by the drop in stress with increasing strain past ultimate stress, entering this negative stiffness in the model resulted in divergence. Therefore, the shear stressstrain curve was updated so that stress does not decrease and the dashed line is used. The UMAT uses eight points from this curve which are chosen by the user to be characteristic of the curve. From this, shear modulus is updated by calculating the secant modulus. 


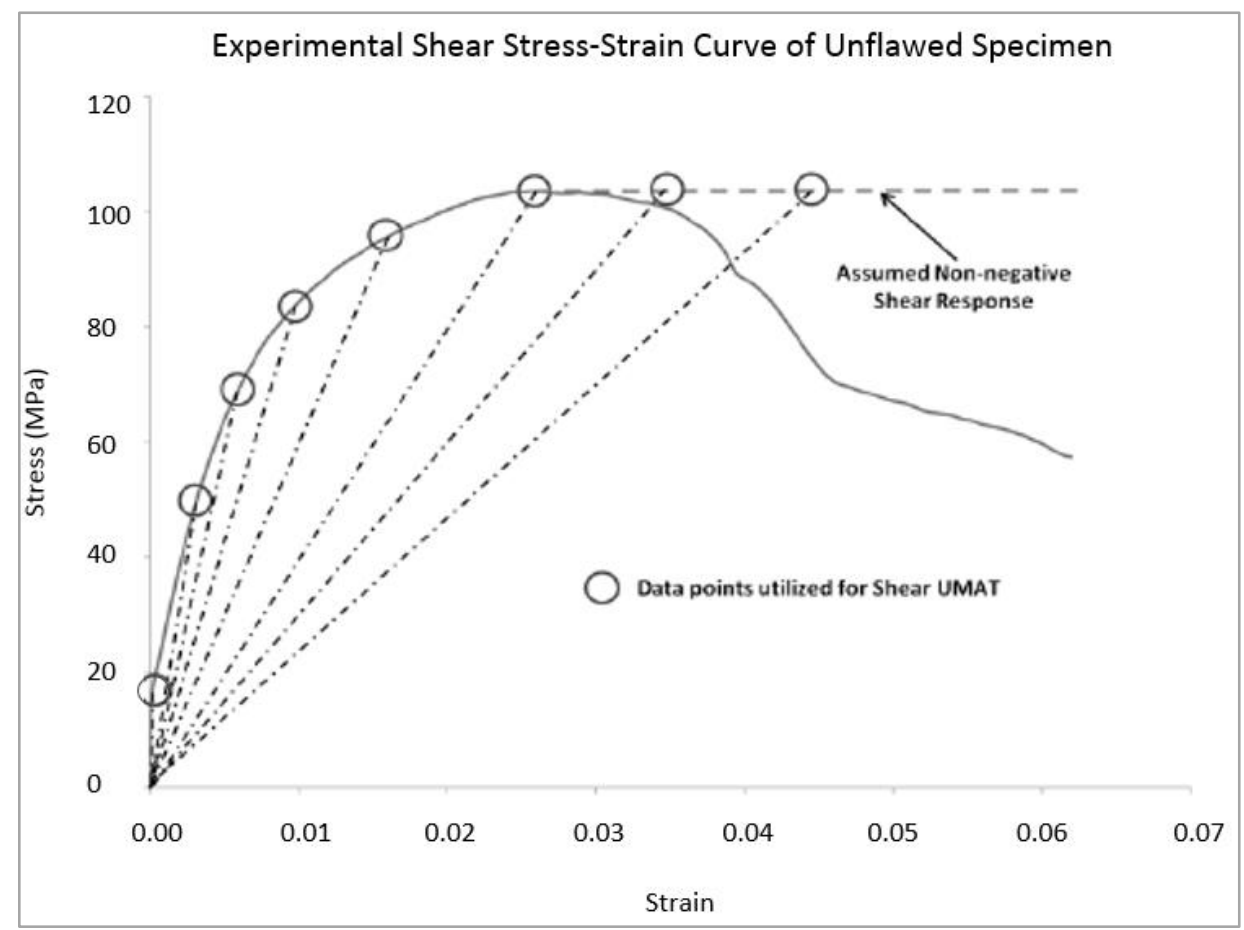

Figure 9: Shear stress-strain response of unflawed specimen used for non-linear shear UMAT. Tabulated data points shown used for UMAT inputs. (Nelson, 2013)

The cohesive element response is based on the bi-linear traction criteria as shown in Figure 10. This relates the separation of the cohesive element to the stiffness. As the cohesive element is pulled apart, the traction increases linearly as defined by its stiffness input $(\mathrm{K})$. Once it has reached a critical, pre-defined stress state $(\mathrm{T})$, the cohesive element is considered damaged. The load carrying capability will then drop with continued separation until the load carrying capability is zero and the element is deleted. The reduction in stress after damage is defined by the energy under the curve $\left(G_{c}\right)$. 


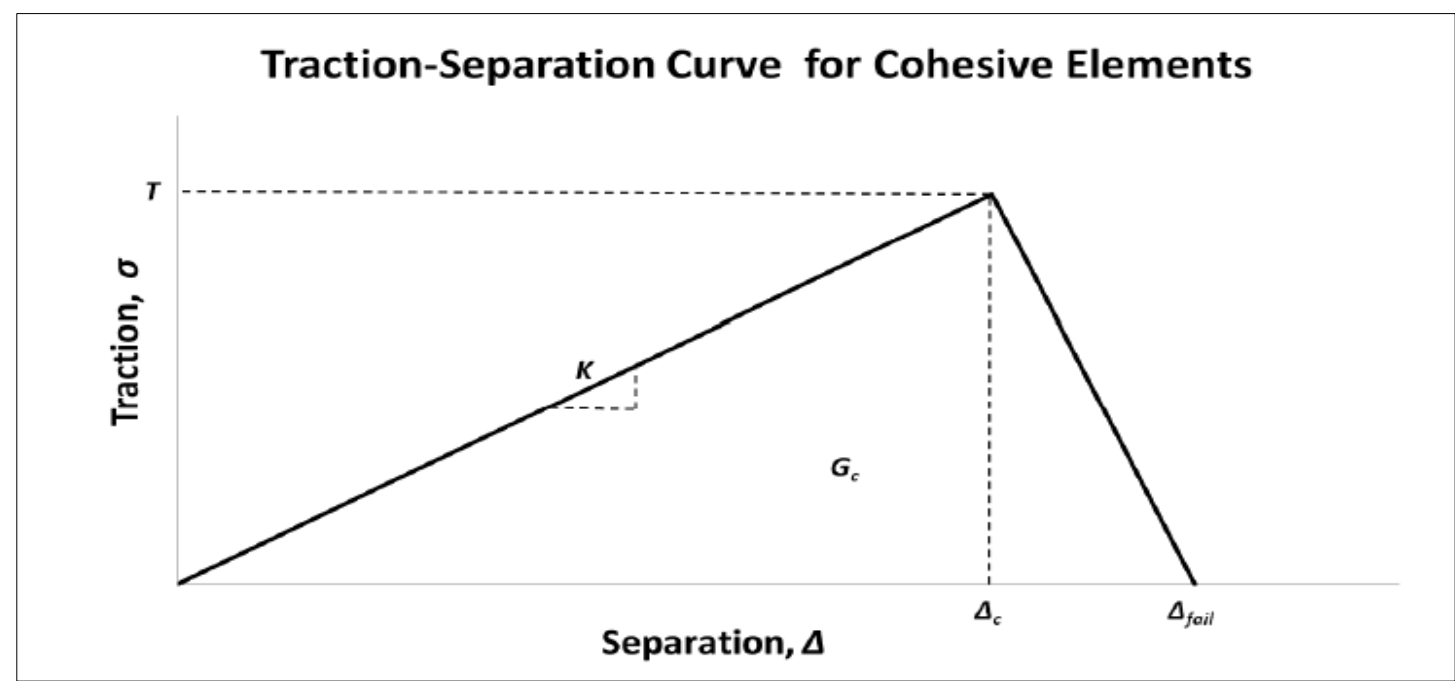

Figure 10: Bi-linear traction-separation criteria defining cohesive element response.

There are a number of material property inputs for both the continuum and cohesive elements. These values are discussed in more detail below in Chapter 4.

A disadvantage of this model is that there can be no failure criteria for the bulk elements. This is because only one subroutine can be used at a time in Abaqus. Because this model uses the non-linear shear UMAT, a subroutine to fail bulk elements cannot be included. This means that it is up to the user to determine when bulk elements have failed.

\section{$\underline{\text { Sandwich Structures }}$}

Sandwich construction is often used in beams and panels in which thin, strong face sheets are bonded to either side of a low density core material as shown in Figure 11. These are used to take advantage of the face sheet strength while adding out-of-plane strength with the core, all while minimizing weight. This results in high in-plane and structural stiffness with the face sheets carrying bending and in plane loads while the core 
stabilizes face sheets and gives out-of-plane shear strength and through-thickness compressive strength (Daniel \& Abot, 2000). Sandwich structures are used in many industries including aerospace, automotive, marine, and wind energy. Face sheets are often metals or composites and the core is often a foam, honeycomb, or balsa. The remainder of sandwich construction background discussed here will be for composite face sheets.

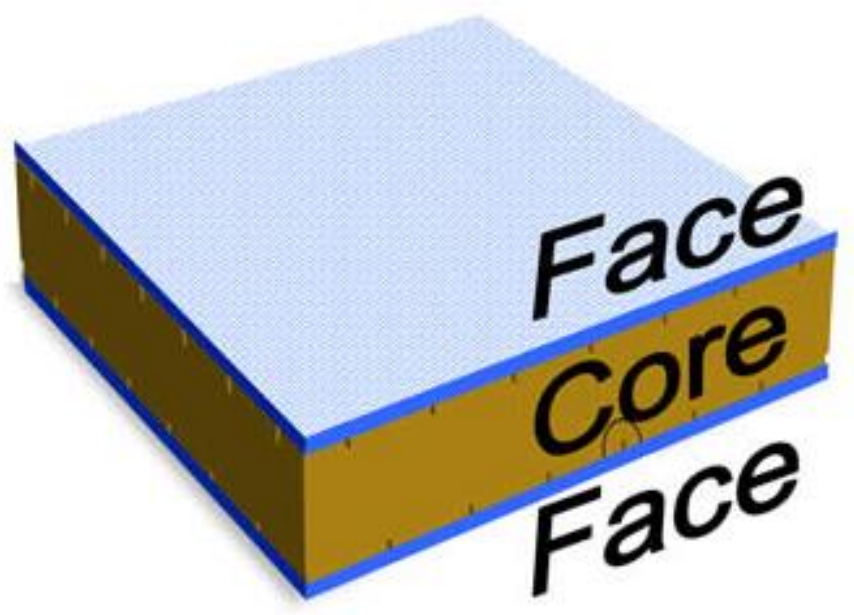

Figure 11: Schematic of typical sandwich structure with two face sheets bonded to either side of a core material. (CompositesWorld, 2014)

Naval vessels and the wind industry are two main users of composite face sheets with balsa cores while other applications often use aluminum, honeycomb, or foam cores for higher shear strengths (Hayman, Berggreen, \& Pettersson, 2007). Balsa and various foam core materials are shown in Figure 12. The third most common type of core is a honeycomb structure that can be made of various materials, most commonly plastics or aluminum. 


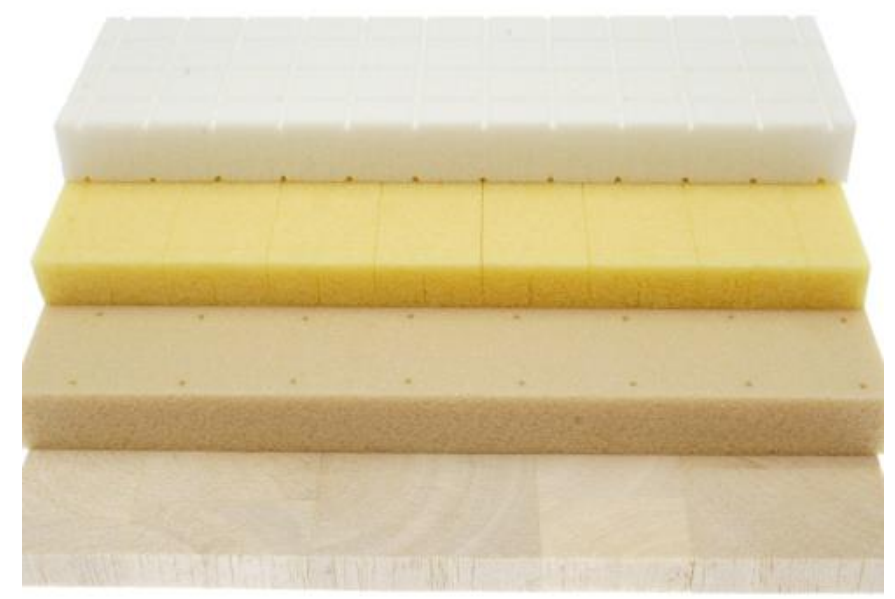

Figure 12: Balsa on bottom and various foam core materials above. (Reinforced Plastics, 2014)

Wind turbine blades utilize sandwich structures in the form of glass fiber reinforced composites and balsa cores. These can often be found in the blade face sheets as well as spar, depending on manufacturer. These parts can be very large considering the size of wind turbine blades. Siemens produces blades up to $75 \mathrm{~m}$ long and weighing 25 tons (Siemens, 2012). Vestas is expected to begin producing 80m long blades in 2015 (Composites World, 2014). There are many types of loading on the blades but bending is considered to be most significant. For this reason, the following background on sandwich structures focuses on flexural loading.

In a typical sandwich structure there are three materials: Composite face sheets, core material, and adhesive between face sheets and core. Any three of these can fail individually, causing global failure. In a typical, symmetric sandwich structure in fourpoint bending, the most likely failure mechanisms are core shear failure, face sheet compressive failure, and adhesive debonding (Manalo, Aravinthan, Karunasena, \& Islam, 2010). The actual likelihood of each of these depends on beam and loading geometry as 
well as materials. Other common failures often caused by loading conditions are indentation failure and face wrinkling (Daniel, Gdoutos, Wang, \& Abot, 2002).

The most common failure mechanism seen in composite sandwich structures in both static and fatigue flexural loading is core shear failure (Daniel \& Abot, 2000), (Manalo, Aravinthan, Karunasena, \& Islam, 2010), (Manalo, Aravinthan, \& Karunasena, 2010), (Gibson, 2011). Core shear failure is an undesirable failure mechanism when trying to investigate the face sheet and novel measures may be required to avoid core shear failure (Daniel \& Abot, 2000).

Previous studies of effects of defects in sandwich beams have focused on wrinkle defects. This is a common defect seen in parts made with resin infusion manufacturing processes (Hayman, Berggreen, \& Pettersson, 2005), (Hayman, Berggreen, \& Pettersson, 2007). A wrinkle defect, seen in Figure 13, is when one or more layers of the composite face sheet is bulges out and can even be folded on itself.

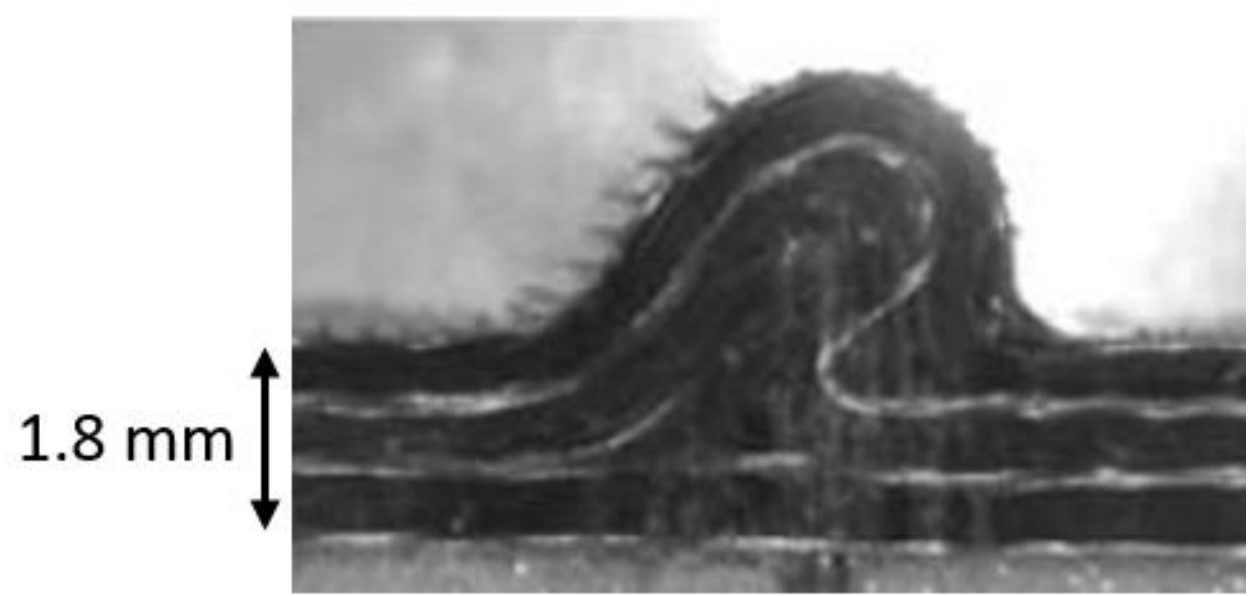

Figure 13: Wrinkle defect example in carbon fiber laminate (Hayman, Berggreen, \& Pettersson, 2005). 
Investigating the effects of defects in sandwich beams for other flaw types is the next step for effects of defects research. There is much coupon level research on IP waves but there is a large gap between the coupon level and full wind turbine blade level. As seen in the testing pyramid in Figure 2 above, the geometry of test specimens must start increasing in complexity. Sandwich beams with fiber waviness is the next step. It is expected that the knockdown caused by fiber waviness in coupons is larger than it would be in more complex geometries. According to previous studies, the core of the sandwich beam, as well as flexural loading, can be expected to increase stability in the plane of the laminate as well as out of plane on the compression side. This stability has been shown to increase the load carrying capability of the unflawed compression face sheet and is expected to increase the load carrying capability of both the tension and compression face sheets with fiber waviness (Wisnom, 1994), (Daniel \& Abot, 2000).

No research into the effects of fiber waviness in a sandwich structure in bending has been found. As mentioned above, researching the effects of defects in sandwich structures in bending is important because the knockdowns used for these sandwich structures come from coupons. Coupon knockdowns are likely too large which is resulting in overdesigned blades. Researching the effect of fiber waviness in a sandwich structure is more applicable and will result in a better understanding of the wind turbine blades.

\section{Overview of Work, Process, and Impetus Summary}

The work herein has a number of seemingly different goals but all are aimed to better understand MSUCG's subtask of effects of defects. These goals are: 
1. Test new type of varying misalignment IP wave in coupon

2. Further develop progressive damage model to model new wave type

3. Develop sandwich beam for four-point bend tests with and without IP waves These actions along with impetus are organized in Table 1 and are discussed in more detail below.

Table 1: Actions completed in this work and impetus for them.

\begin{tabular}{|l|l|}
\hline \multicolumn{1}{|c|}{ Action } & \multicolumn{1}{c|}{ Impetus } \\
\hline $\begin{array}{l}\text { Test new type of varying misalignment IP } \\
\text { wave in coupon }\end{array}$ & $\begin{array}{l}\text { Investigate more typical IP wave with } \\
\text { fibers of varying misalignment giving } \\
\text { insight into interactions between fiber } \\
\text { tows caused by misalignment gradient } \\
\text { across width }\end{array}$ \\
\hline $\begin{array}{l}\text { Further develop progressive damage } \\
\text { model to model new wave type }\end{array}$ & $\begin{array}{l}\text { Improve robustness of previously } \\
\text { developed progressive damage model and } \\
\text { verify test results }\end{array}$ \\
\hline $\begin{array}{l}\text { Develop sandwich beam for four-point } \\
\text { bend tests with and without IP waves }\end{array}$ & Investigate effects of defects in flexure \\
\hline
\end{tabular}

The impetus for the actions listed above are now summarized. The new type of IP wave coupon is introduced because the previous IP wave coupon is not considered typical of IP waves seen in manufactured parts. The complex interactions between fiber tows of differing wave parameters is deemed a necessary topic to investigate because this is more similar to IP waves seen in manufactured parts. Therefore, the new IP wave coupon is a 4 ply unidirectional coupon, $\left[(0)_{4}\right]$, same as before, except it has straight fiber tows on either side. The wave fades in from straight tows and fades back out to straight 
tows as discussed below. This new wave will also avoid the premature failure at $0.5 \%$ strain caused by discontinuous edge fibers.

The second goal is modeling this IP wave coupon using the combined model approach previously developed by MSUCG. The goal of the model is to be an accurate and predictive progressive damage model. While it has previously shown promising results in various wave types and materials (Nelson, 2013), continuous improvement is necessary to get this model to the accuracy and predictive capabilities desired. Continuing to model new types of waves increases the robustness. Further, other changes are suggested to increase the accuracy. This process gives insight into the exact mechanisms causing failure, shedding more light on the new IP wave coupon.

The third goal is to develop a composite face sheet, balsa core sandwich beam for four-point bending. The purpose of developing this test specimen is to investigate the effect of a stabilizing core material. There has been much research on the effects of defects at the coupon level but the complexity of specimen and loading geometry must be increased to work toward the full wind turbine blade. This specimen will start to fill in the gaps. The effect of the core material is expected to be stabilization of the face sheets, increasing load carrying capability in the unflawed compression face sheet and in both compression and tension face sheets with IP waves. Due to this, the knockdown caused by fiber waviness is expected to be smaller in sandwich beams than in coupons which will result in less overdesigned blades. The sandwich beam goals are not to carry out comprehensive testing with these beams but to develop the test specimen to enable a comprehensive test program. 


\section{CHAPTER 3}

\section{COUPON DEVELOPMENT AND TESTING}

\section{$\underline{\text { Overview }}$}

As mentioned above, the scale of effects of defects research is increasing to bridge the gap between the coupon level and full-scale wind turbine blades. To do this, an upcoming focus of MSUCG is effects of defects in sandwich beams. While this will give more applicable data it will also be much more expensive monetarily and temporally. Before the scale of research is shifted to the sandwich beam scale, more research can be accomplished on the coupon level. Extensive coupon testing has already been performed with the IP wave coupons with waves of constant amplitude extending across the whole width. The next step before sandwich beams is to increase the complexity of these wave coupons by including changing wave severity across the width which includes straight fibers on either side of the wave. This chapter includes more of the impetus as well as design and manufacturing of these coupons. The testing procedure and discussion of these coupons, including a comparison with the previous constant amplitude IP wave coupons, are also included.

\section{Impetus and Design}

Testing for the effects of defects subtask of MSUCG has so far been based on the coupon level of testing. Flaws investigated have been porosity, out-of-plane waves, and in-plane waves. The work herein builds off of the in-plane wave coupon testing already 
performed. Previous IP waves were made in such a way that discontinuous fibers lined the edges, causing premature, localized failure as shown in Figure 7 above. Although this damage did not cause global failure, this initial failure of the discontinuous fibers caused an asymmetry resulting in a bending moment about the through-thickness axis. IP waves seen in composite parts are not typically of this geometry. As seen in Figure 4 above, the waves are built up through the width of the part rather than continuous through the width. This means that the progressive damage and failure of waves in manufactured parts are likely also different than has been found in previous work. By making each fiber tow contain the same wave parameters, the complex interactions caused by changing wave parameters between tows, as in manufactured parts, has not been investigated. Therefore, there was a need for a new, more applicable wave coupon to be developed, tested, and modeled before moving up in scale.

As mentioned above, waves in manufactured parts typically fade from straight fibers into wavy fibers and back to straight fibers. This was taken into account for the design of the new IP wave coupon in which fibers along each edge are straight. Moving in from the edges, waviness gradually increases in each fiber tow into the middle fiber tow which has the largest wave. The wave section of this manufactured coupon can be seen in Figure 14. Due to the wave severity gradient, stress and strain distributions are not expected to be constant across the width as seen in previous DFIP coupons. The complex strain distributions are discussed further in Chapter 3 and Chapter 4. 


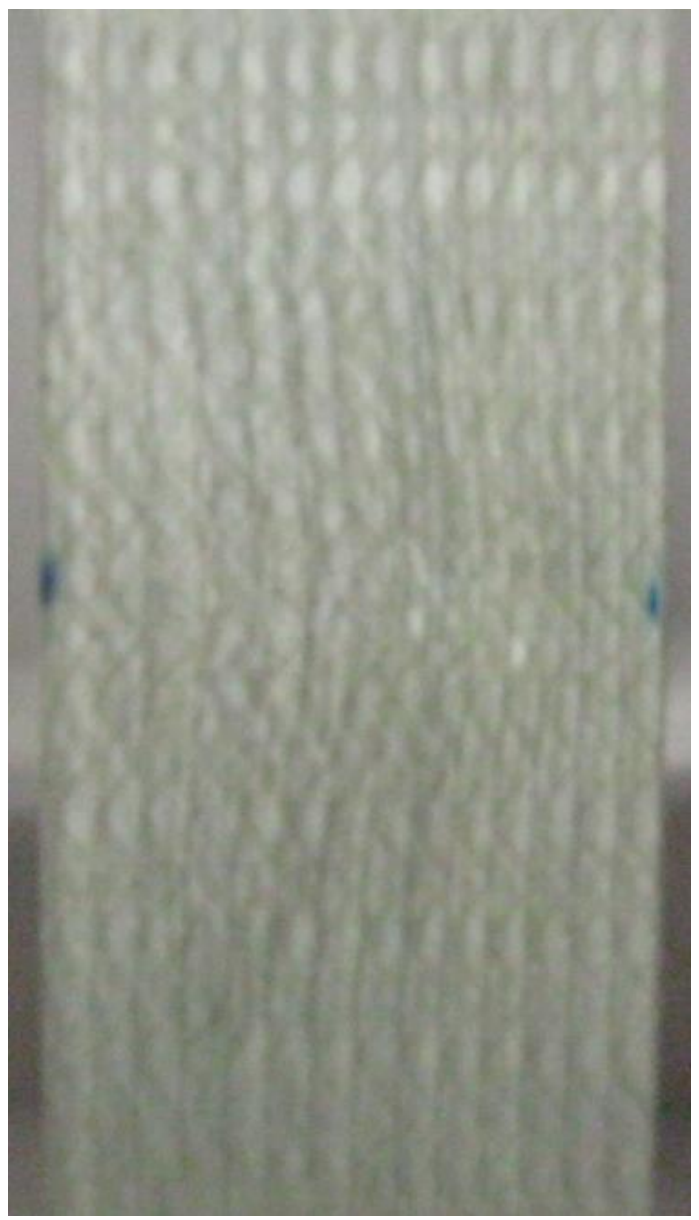

Figure 14: Middle portion of continuous fiber IP (CFIP) wave coupon held up to light. Marks on either side indicate the location down the length of maximum wave amplitude.

A schematic showing the fiber tows for this continuous fiber coupon wave type is shown in Figure 15. A fiber tow schematic for the previous wave coupon with discontinuous edges from Figure 6 above is also included for comparison. The discontinuous fibers seen in the right coupon within the wave region are highlighted in the right oval. The coupon on the left, however, has continuous fibers in the same region. The previous IP wave coupon will be called the discontinuous fiber in-plane wave (DFIP) due to the discontinuous fibers, whereas the new coupon will be referred to as continuous fiber in-plane wave (CFIP). 


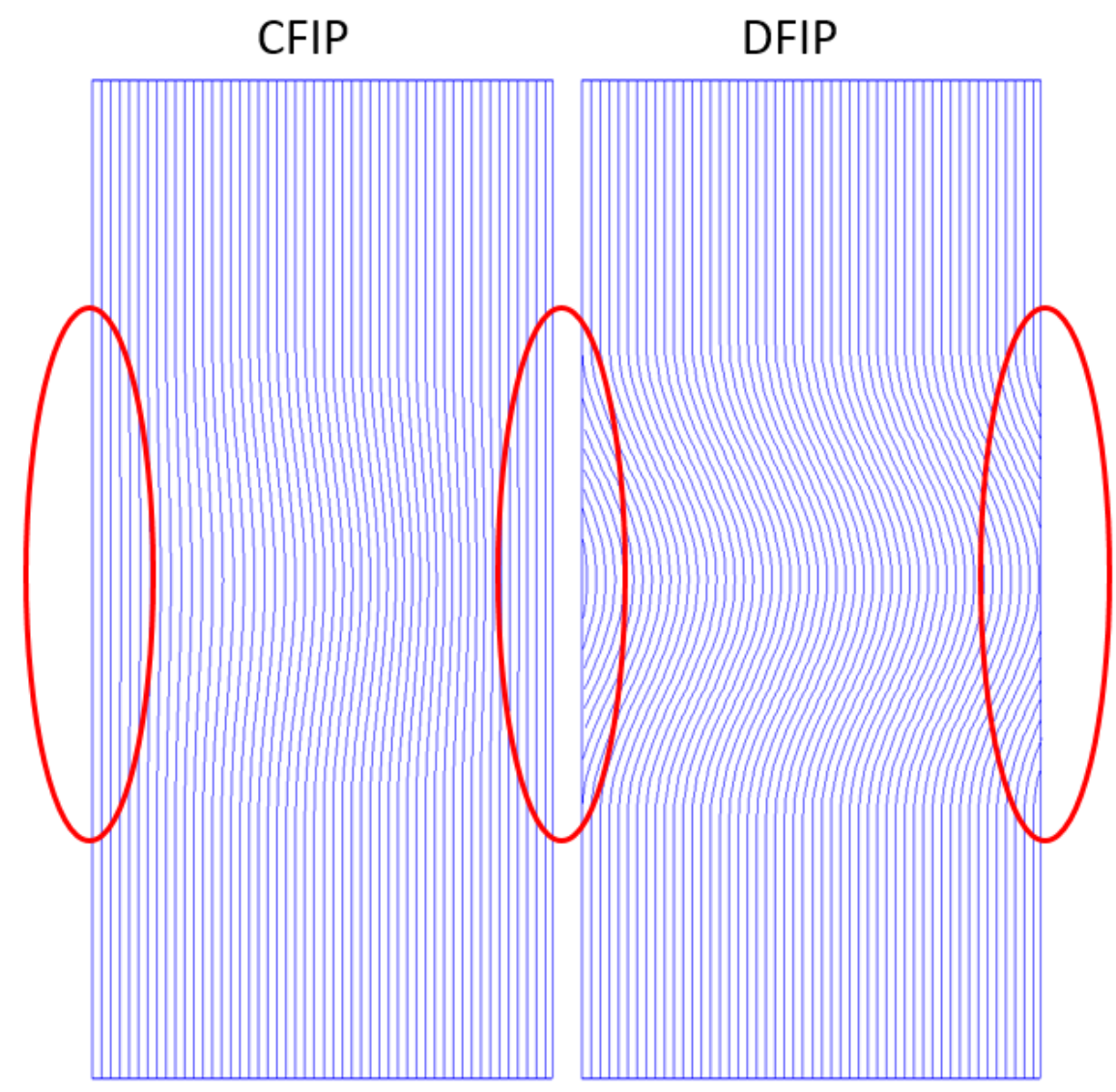

Figure 15: Schematics of fiber tows in coupons of both wave types, continuous fiber IP (CFIP) wave coupon (left) and discontinuous fiber IP (DFIP) wave coupon (right). Main difference of continuous and discontinuous fibers can be seen by comparing the edges within each wave section shown with the red ovals.

The wave in each fiber tow in the discontinuous fiber IP (DFIP) wave coupons was similar. Therefore, the characterizing wave parameters previously introduced (wavelength, amplitude, average misalignment angle) applied to all fiber tows. The continuous fiber IP (CFIP) waves have fiber tows of varying wave severity and, thus, varying parameters, across the width. In the continuous fiber IP (CFIP) waves, the wave parameters are used to characterize the middle, most misaligned fiber tow. Therefore, average misalignment angle is now maximum misalignment angle. The other control on the continuous fiber IP (CFIP) wave is keeping edge fiber tows straight. This introduces 
the potential for additional characterizing parameters as discussed below but left as future work. It should be noted that the orientation of the waves in both Figure 14 and Figure 15 above, in which the fibers are displaced to the right to form the wave, is kept consistent throughout the work herein for ease of discussion. Hence discussion of "left side tows" and "right side tows" are specific to this orientation which is kept consistent throughout.

The wave parameters were desired to be similar to the previous research with discontinuous fiber IP (DFIP) wave coupons. Therefore, a wavelength of $50 \mathrm{~mm}$ was desired. However, matching amplitude and misalignment angle was not possible with the continuous fiber IP (CFIP) wave type as discussed below. The maximum amplitude for all continuous fiber IP (CFIP) coupons tested range between 4 and 10 degrees. The amplitude for all continuous fiber IP (CFIP) coupons was around $3 \mathrm{~mm}$.

\section{Coupon Manufacturing}

All coupons used for the research herein and previous MSUCG research which this is building upon were made with the vacuum resin infusion process. Previous works are referenced in Chapter 2 for more details on this process but because it is important to research herein, it is included in detail here as well.

The basic vacuum resin infusion process is the same for all control and IP wave coupons and is as follows. An aluminum mold is first prepped with mold release and left to dry for 20 minutes. The layers of material used for this process are two layers of peel ply, one layer of flow media, a vacuum bag, and four layers of glass fiber mat. The layup process can be seen in Figure 16. First, tacky tape is placed along the perimeter of the 
mold to secure the vacuum bag after lay-up. For lay-up, the first material on the mold is one peel ply, followed by the glass mats. The desired coupon lay-up is $\left[(0)_{4}\right]$ so 4 unidirectional glass mats are used, with fibers aligned and backing facing toward the laminate midline. This point is shown in Figure 16 (left). The glass is then covered with another layer of peel ply, flow media, and then the vacuum bag. The mold after lay-up and under vacuum can be seen in Figure 16 (right).
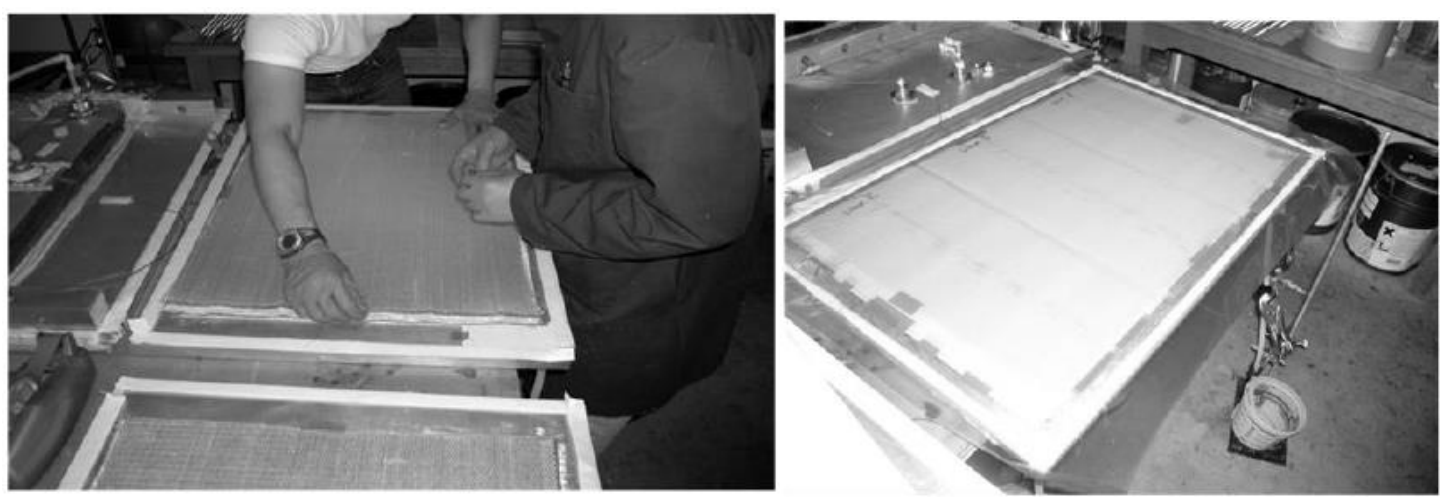

Figure 16: Preparing mold to inject resin to make laminate using vacuum resin infusion process. Laying materials on mold (left) and finished lay-up under vacuum (Nelson, 2013).

Once vacuum has been pulled, and it is apparent that there are no leaks, resin can be injected. The resin and hardener are first measured, combined, mixed, and degassed. Once the resin is prepped, it is injected through a spiral tube on one side of the laminate, perpendicular to the glass fibers. The resin is then pulled through the glass fibers, parallel to the fiber direction, towards the vacuum port.

The IP wave coupon manufacturing process is the same as the vacuum resin infusion process outlined above except that the layers of fabric are prepped with wave geometry before lay-up on the mold. This process is explained in detail in Nelson, 2013. The discontinuous fiber IP (DFIP) wave introduction process is as follows. One layer at 
a time, the glass fabric is laid over a polyethylene tube oriented transversely to the tow direction, creating an OP wave. Steel bars are set on either side of the wave and the tube is removed. An image of the steel bars and OP wave is shown in Figure 17.

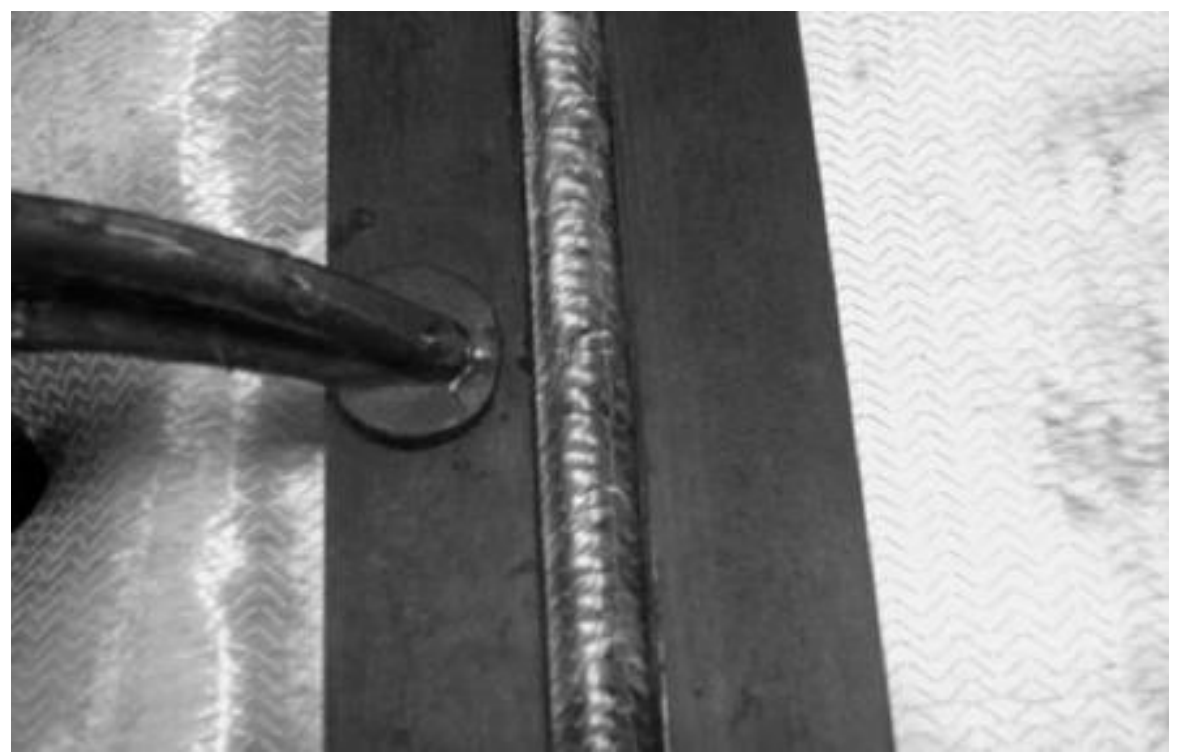

Figure 17: Glass fabric laid over tube to form OP wave with steel bars on either side.

The OP wave is then rotated over into the plane by dragging and pressing. The bars are removed and the glass fiber mat is placed under a glass plate to help retain the wave shape while the fibers relax. This is repeated for all the plies and then the vacuum resin infusion process is continued as usual, making sure to align the waves in each ply.

Adding the continuous fiber IP (CFIP) wave is more involved than the discontinuous fiber IP (DFIP) wave. The same idea of forming an OP wave and rotating into the plane is used for forming continuous fiber IP (CFIP) waves. However, the method with the tube results in a continuous, constant amplitude wave through the laminate. Because each tow in continuous fiber IP (CFIP) coupons are not the same amplitude, putting in a wave for a whole ply in one step is not possible. Coupon 
locations in each layer are chosen and wave introduction locations are determined from this so that the wave is in the middle of each coupon. Varying amplitude IP wave also means that the OP wave form cannot form a continuous amplitude wave. This means that the removal of the wave form cannot be as simple as pulling from the side because glass fibers will enclose the wave form.

The amplitude must increase from zero displacement to a maximum value and back to zero over a $50 \mathrm{~mm}$ span. This requires a wave mold rather than a tube to induce this OP wave. A wave mold was designed in SolidWorks to form waves with desired parameters as discussed above. This can be seen in Figure 18. The manufactured part is shown in Figure 19 (left). The marks are so that it does not get lost as easily, being made of a translucent material. The width of this part is $40 \mathrm{~mm}$ so that $5 \mathrm{~mm}$ of fibers on either side of the wave are not perturbed and will remain straight. The length is $50 \mathrm{~mm}$ so that the wavelength is $50 \mathrm{~mm}$. The amplitude of the wave is determined by the length of the curved surface over which each tow lies. The difference between this length and the $50 \mathrm{~mm}$ wavelength is the extra tow length that can be rotated into an IP wave. The wave mold in use can be seen in Figure 22 below.

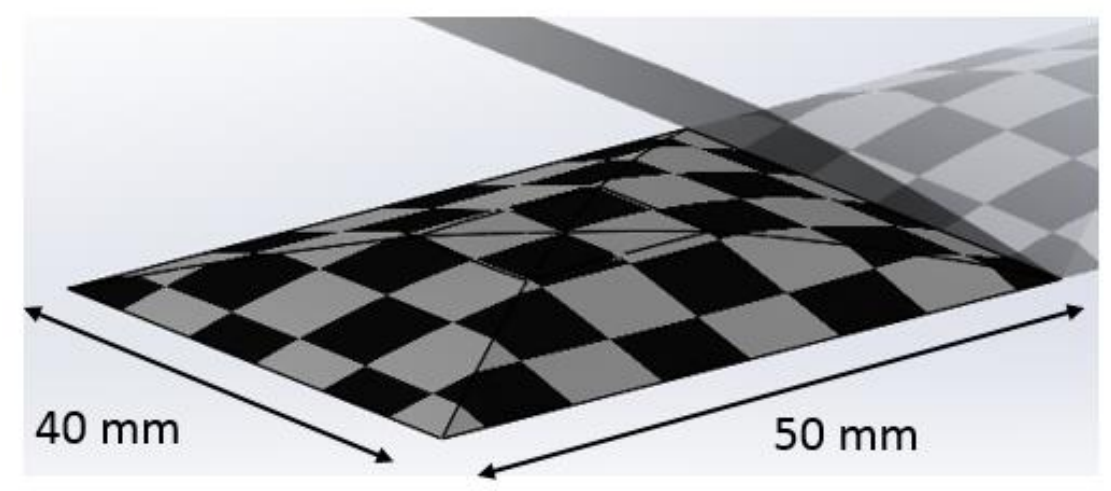

Figure 18: Wave form for continuous fiber IP (CFIP) waves as designed in SolidWorks. 

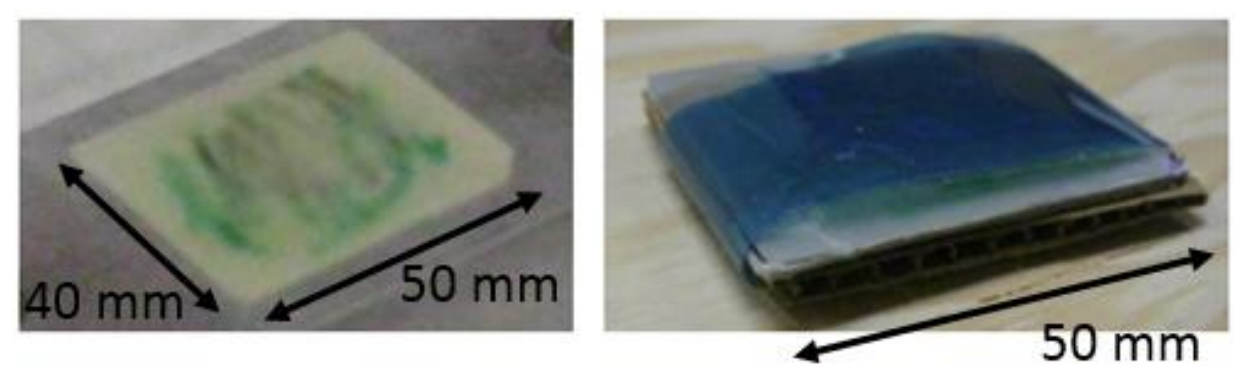

Figure 19: Wave mold as made (left) and modified by increasing height to increase wave amplitude (right).

The amplitude from this wave form was found to be too small. Rather than redesign this part and make it again, the existing wave form was modified by taping cardboard underneath, as can be seen in Figure 19 (right).

The next task in developing the continuous fiber IP (CFIP) wave was the ability to remove the wave mold. Whereas for the discontinuous fiber IP (DFIP) wave, the tube could be removed from the side, this wave form is fully enclosed by glass. This means that the wave form must be removed from the bottom to not alter the glass after wave introduction. This requires a table top with a removable portion. This is made of wood and has a $50 \mathrm{~mm}$ wide strip extending across the width that drops down, allowing the wave mold to be removed from underneath. This is shown in Figure 20. The dimensions are 3 by $6 \mathrm{ft}$. While this size is excessive for these laminates, it is made large enough for putting waves into beams. Images showing the process of removing the middle strip can be seen in Figure 21. The middle strip is resting on removable blocks on either side. When the blocks are removed the strip drops down. 


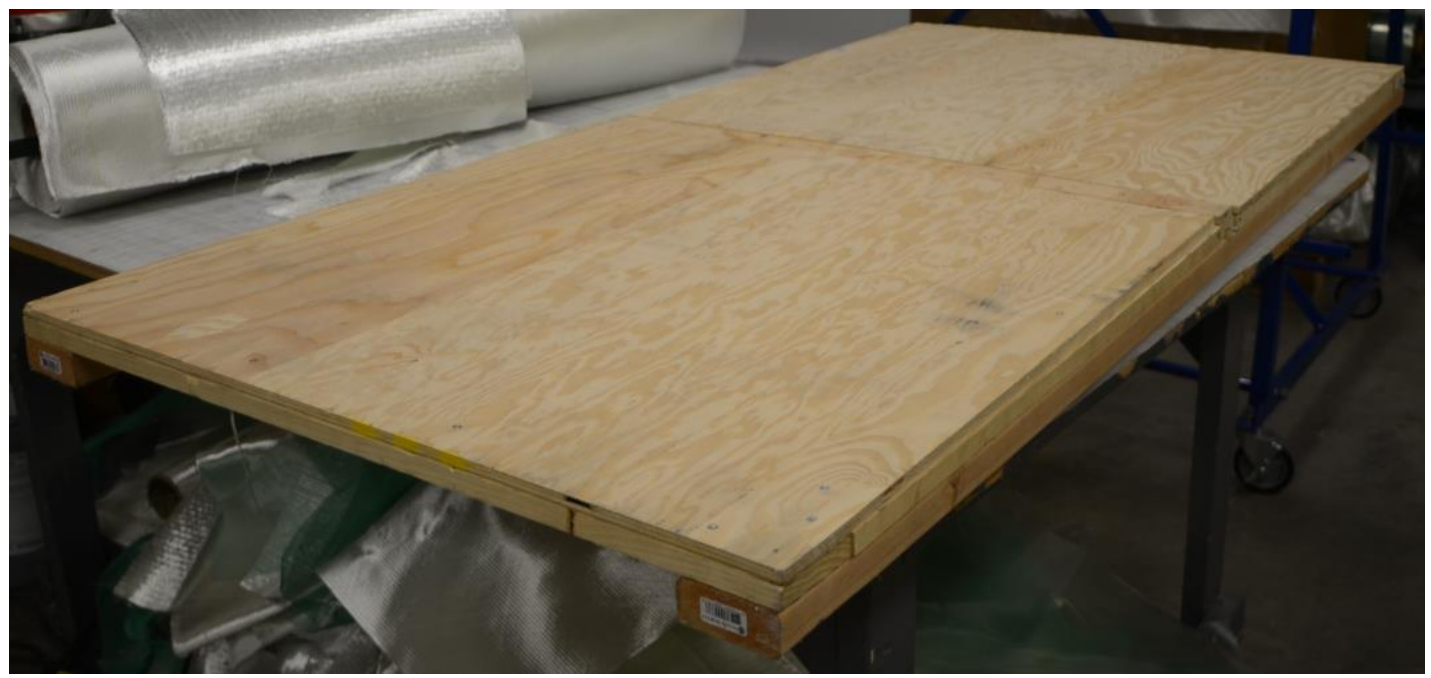

Figure 20: Table top with removable section for continuous fiber IP (CFIP) wave introduction.
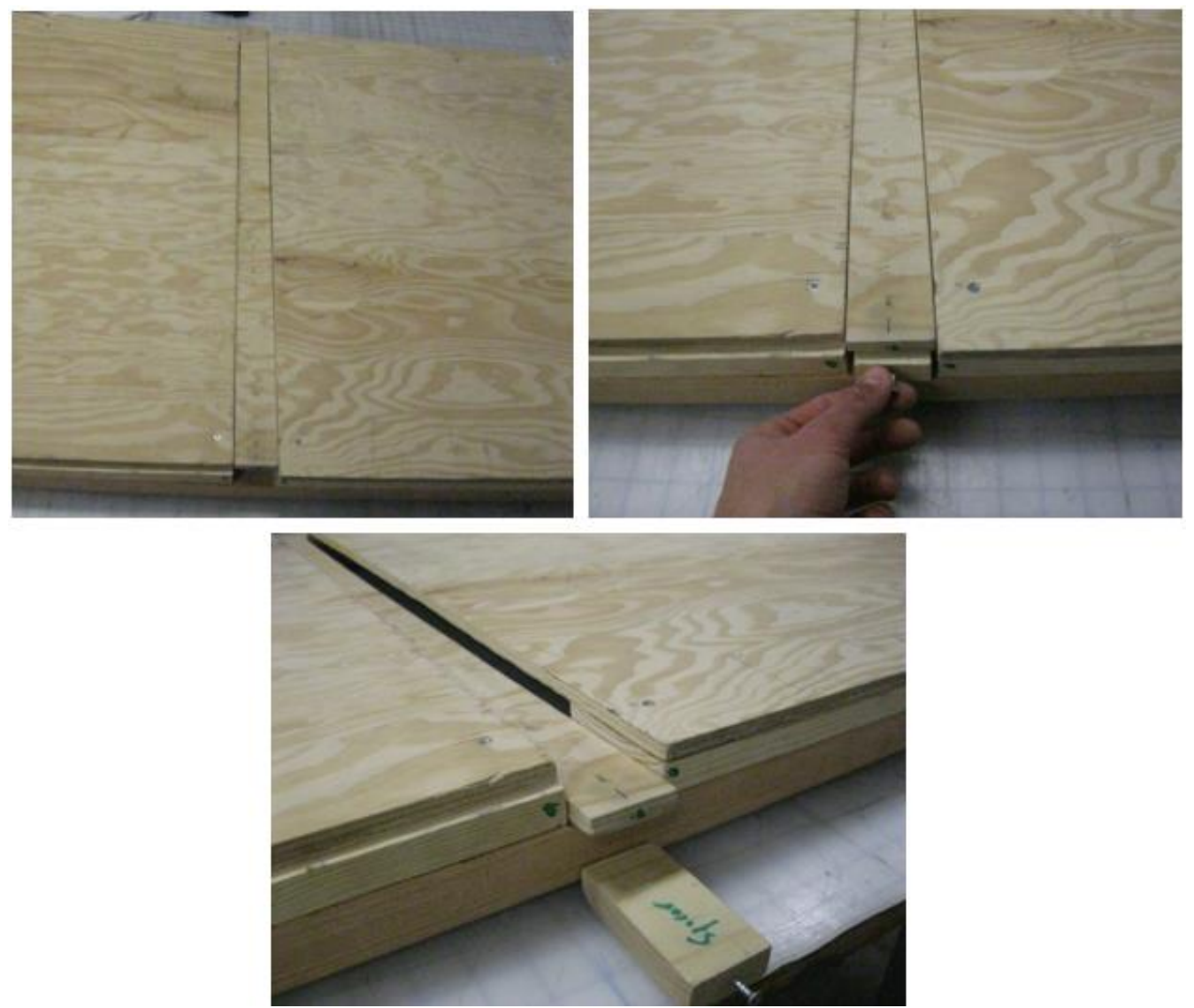

Figure 21: Table top middle strip. Removable middle strip (top left), spacer being removed (top right), middle strip dropped down after the spacers have been removed (bottom). 
The wave introduction process starts with locating where the waves will be in the glass fiber mat so that the waves will align when laid-up. The wave form is then placed on the removable strip on the table top. The glass fiber mat is placed over this, aligning the wave location with the wave mold. Steel bars are placed on either side of the wave, parallel to the removable strip. The wooden spacers underneath the removable strip are removed, allowing the strip to drop down. The strip is then slid out so that the wave mold can be removed. Once the wave mold is removed, the removable strip and spacers are replaced. The OP wave in the fibers can then be dragged and pushed to rotate the OP wave into an IP wave. This is done with a combination of fingers and a wood block with a corner sanded round. These steps are shown in Figure 22, in order from left to right and top to bottom, as indicated by the arrows. This is repeated for each wave in the mat. Once all the waves in a mat have been formed, the fibers are placed under a heavy glass plate to help retain the shape. This is then repeated for the other glass fiber mats. The rest of the manufacturing steps are the same as for the discontinuous fiber IP (DFIP) laminate except that now even more care must be taken to align the waves between fiber mats because there is now an extra degree of freedom for wave alignment. This is controlled by making precise, consistent measurements on the fiber mats for where to form the wave as well as making sure to align the fiber mats properly on the mold. 


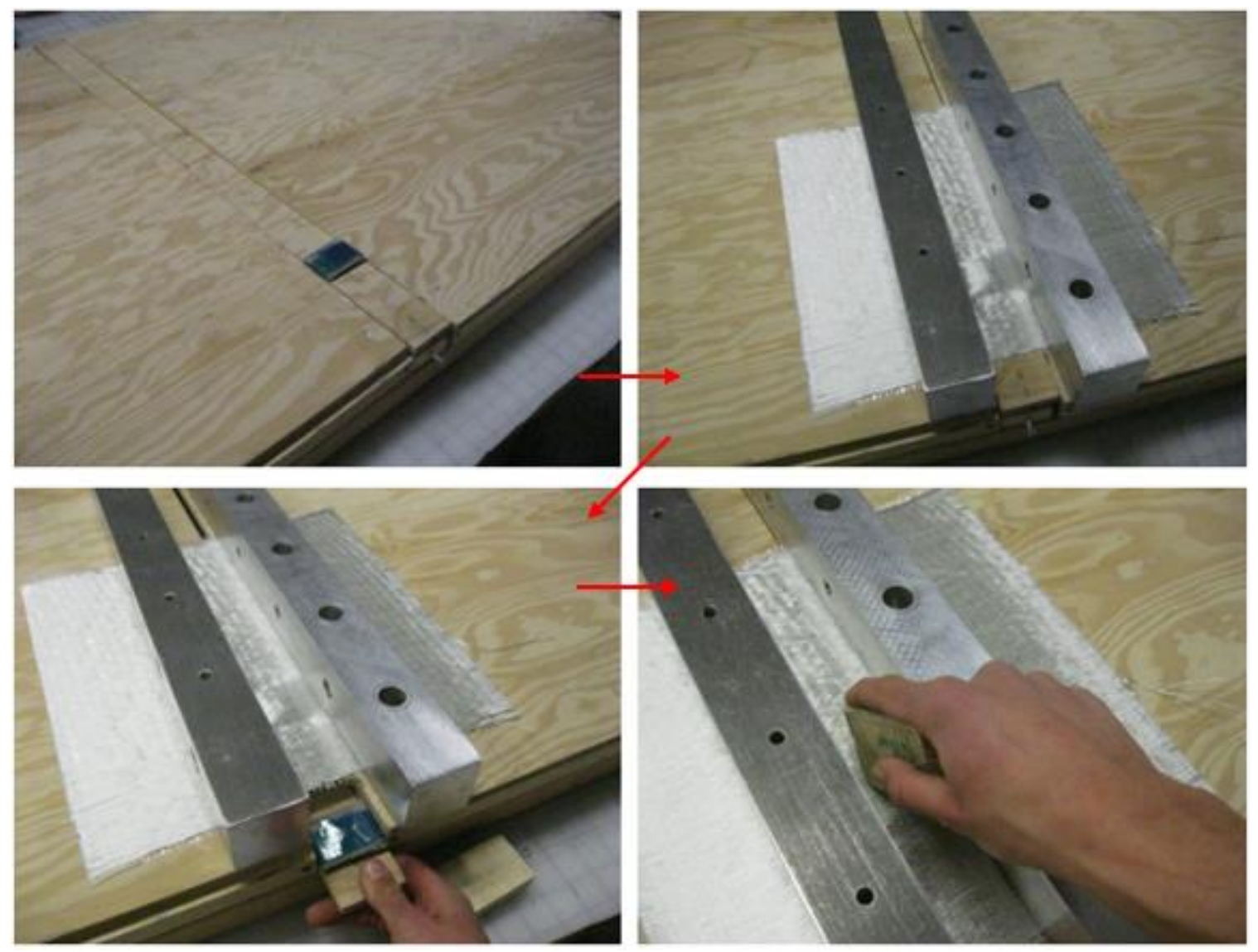

Figure 22: continuous fiber IP (CFIP) wave introduction. Wave form in place (top left), glass mat over wave form with steel bars (top right), wave form removal (bottom left), $\mathrm{OP}$ wave rotating into IP wave using rounded block (bottom right). Arrows indicate order of steps.

The materials used for the continuous fiber IP (CFIP) coupons are PPG 1250 UD 2026 glass and the resin and hardener are Hexion Epikote MGS RIMR 135 and RIMH 1366 respectively. These are the same materials used for the discontinuous fiber IP (DFIP) coupons except that they have different sizings on the fibers. The previous wave research used glass fibers with 2002 sizing rather than 2026. Little information is public knowledge on these sizings. The purpose of sizing is for bonding between the glass fibers and resin. Any effect of a different sizing would be related to the strength at the fiber-resin interface. This could cause fiber pullout damage to be more or less likely. 
However, because these failures have not been seen in either types of coupons, the sizing has been determined to have a negligible effect. For the purpose of this work, the materials can be considered the same and material responses will be compared accordingly.

\section{Coupon Wave Parameters}

Previous MSUCG work with the effects of defects investigated IP waves with various average misalignment angles but the bulk of the work was with waves with 29 degree average misalignment angle. While it would be desired to continue with the same misalignment angle, the displacement required to achieve this angle would add too many variables to the test coupon as discussed here. By having the wave fade in and out, each side of the wave has a slight out-of-plane portion, one side thinner and the other thicker, as expected when pushing material from one side to the other. The larger the wave, the larger the difference between these two thicknesses. Because this investigation is for IP waves, an OP portion is undesired and should be minimized. This is minimized by implementing less severe IP waves.

The wave was designed to have the same wavelength as the previous IP waves studied. The amplitude desired to balance severity of wave with out-of-plane portion is just under a fiber tow width of $4 \mathrm{~mm}$. Based on viewing the outer plies, these came out to be about 2 to $4 \mathrm{~mm}$. Maximum misalignment angles were between 4 and 10 degrees. The maximum misalignment angle is the angle of the most misaligned fiber tow in the middle of each coupon. 
Four laminates with four continuous fiber IP (CFIP) wave coupons in each were made. One of the laminates used a caul plate to investigate the effect of on reducing the out-of-plane portion. The out-of-plane reduction was minimal and coupons from this plate tested similar to others. Further discussion on these coupons is included below.

CT scans were performed on coupons from the first two plates to check the wave parameters of the inner plies. It is desired that the waves in each ply have similar parameters and align through the thickness which would mean that the manufacturing method developed is working as planned. After reviewing the CT scan images, it appears that there is consistency between the waves in each layer. The CT scans can give a resolution through the thickness down to $0.3 \mathrm{~mm}$. Because the thickness outside the wave for each ply is no less than $0.7 \mathrm{~mm}$, this is enough resolution to investigate each ply individually. However, as mentioned above, the wave portion of the coupon has a slight OP portion. This means that the thickness of each ply in the wave section is larger on one side and smaller on the other. These thicknesses are also slightly different from the far-field thickness. This makes it impossible to include an image of each ply individually because cross-sections can be taken at a certain depth but each ply has varying depth in the wave section. An image of the tool side ply can be achieved because it has one side completely in-plane. This is shown in Figure 23 (left) for a typical coupon. The view angle is through-thickness, which is the same as Figure 14 above. 


\section{Single Ply}

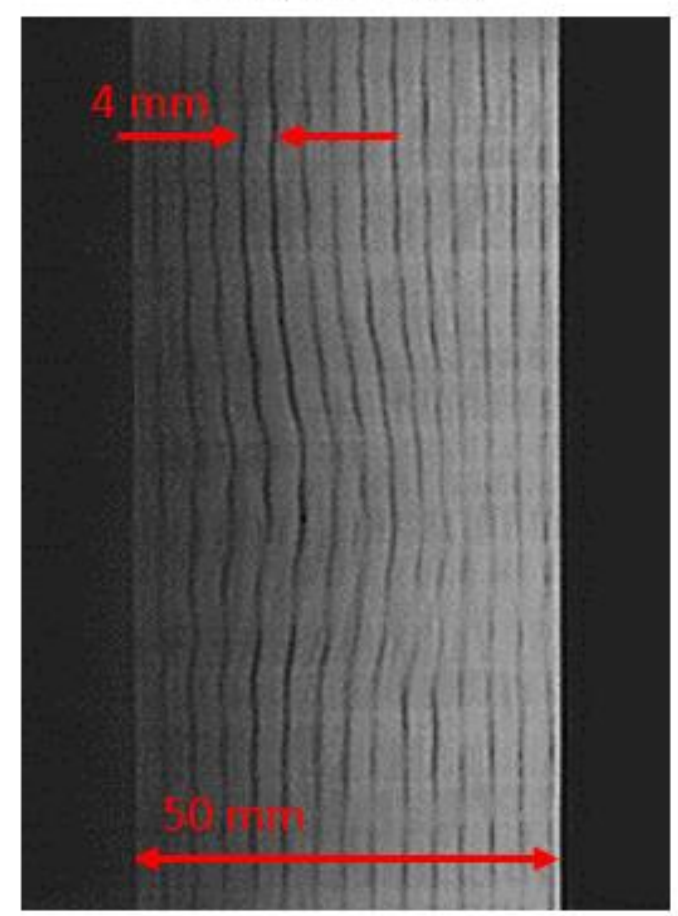

\section{Through Thickness}

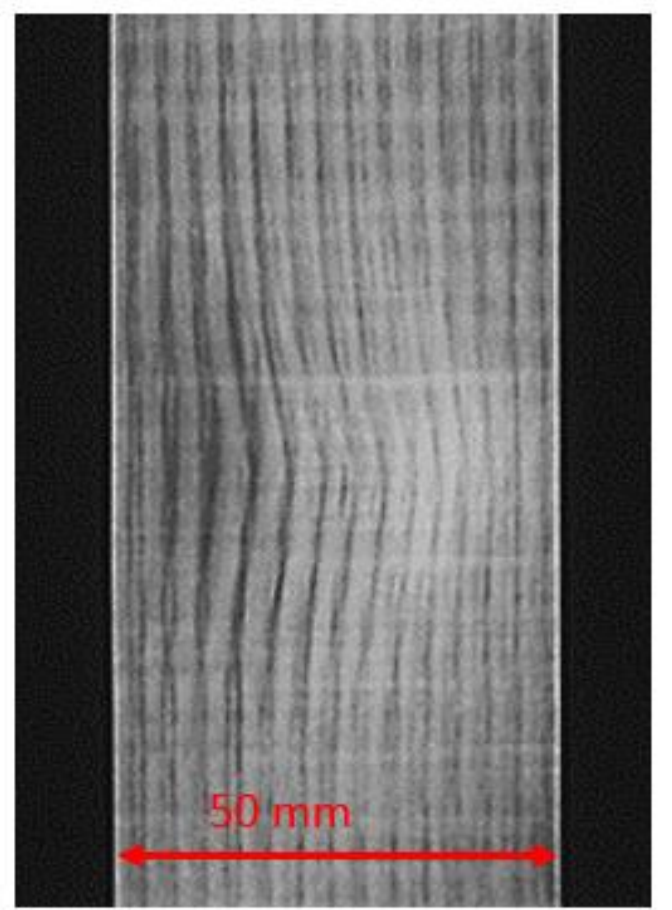

Figure 23: CT scan of tool side ply of typical continuous fiber IP (CFIP) wave coupon (left). CT scan averaged over whole thickness of typical continuous fiber IP (CFIP) wave coupon (right).

Rather than include an image of each ply, Figure 23 (right) is an image of a typical coupon that is averaged over the whole thickness of the coupon. This image includes all layers of the coupon. It can be seen that the wave is clearly visible with a well-defined maximum amplitude. Because this image is an average, good definition means that the layers are well aligned and have similar wave properties.

The maximum amplitude for all plies in all coupons came out to be around between 2 and $4 \mathrm{~mm}$. The wavelength was desired to be $50 \mathrm{~mm}$, to be the same as previous IP wave coupons. The wavelengths came out to be between 50 and $60 \mathrm{~mm}$. This is not crucial because, as mentioned above, the critical flaw parameter is the 
misalignment angle, not wavelength. The misalignment angles for all coupons measure with CT scan images are between 4 and 10 degrees.

The OP portions of these waves can be seen with a cross-section looking down the length of the coupon, shown Figure 24 (left). Coupon thickness is horizontal, width is vertical, and length is through the page. This can be compared to a similar cross-section taken outside of the wave region, as seen in Figure 24 (right). The thickness variation can change by about $0.5 \mathrm{~mm}$ in either direction from the far-field thickness that averages $3.7 \mathrm{~mm}$ for all coupons. This OP variation is small and, as seen in modeling, has a negligible effect on the response.
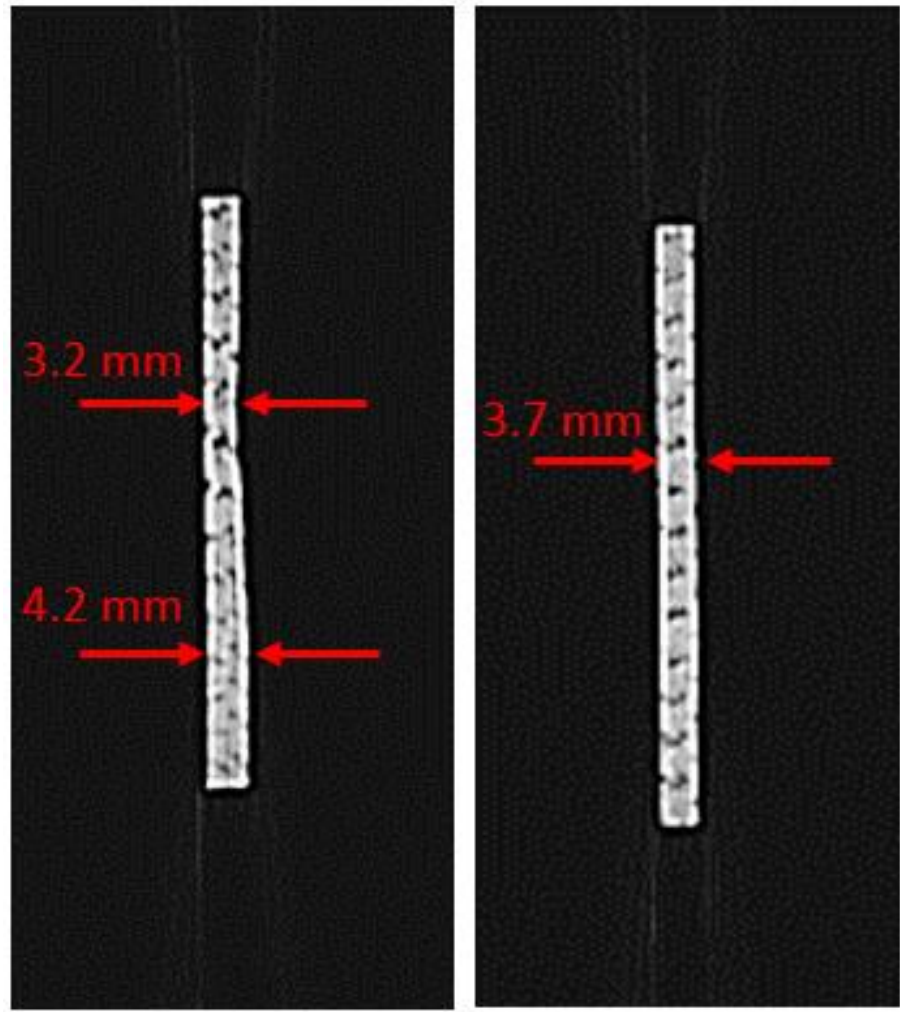

Figure 24: CT scans of cross-sections of continuous fiber IP (CFIP) wave coupon both through the wave portion (left) and outside the wave (right). Thickness variation in the wave can be seen with thickness dimensions labeled at maximum and minimum (left) and a constant thickness can be seen down the length, outside of the wave (right). 


\section{Coupon Testing}

The Instron $8802250 \mathrm{kN}$ test machine and grips are used for coupon testing. The coupons are loaded in tension using displacement control set at $0.001 \mathrm{inch} / \mathrm{second}(0.025$ $\mathrm{mm} / \mathrm{second}$ ). The strain and displacement data are captured using the Aramis v6.2 system which uses digital image correlation (DIC). The test setup, including Instron and Aramis, is shown in Figure 25. The Aramis requires all test specimens to be painted white and then speckled with black paint. The speckling pattern grain size is chosen based on the size of the volume being measured. An image of a coupon with the speckle patter is shown in Figure 26. This system utilizes two 5 megapixel cameras to track the displacements of the speckle pattern in and out of the plane using stereoscopy. After testing, the software calculates strains from the displacements. Testing was performed in two sets. The first set, called round 1, used the Aramis cameras oriented horizontally, as seen in Figure 25 (left). The second set of testing, called round 2, used the Aramis cameras oriented vertically as seen in Figure 25 (right). The reason for camera rotation is discussed below. Images were set to be taken every 1 second for round 1 testing and 2.5 seconds for round 2 testing. These time differences do not affect results because both imaging frequencies are much less than the time of the tests. Also, both frequencies are small enough to capture damage as it occurs up to final failure, at which point cascading damage and failure is too fast to be captured by either imaging frequency. More information on the Aramis measuring system can be found at Trilion Quality Systems (Trilion) and more information on using this system can be found in Parker, 2009. Load data output from the Instron is also recorded with each image. 

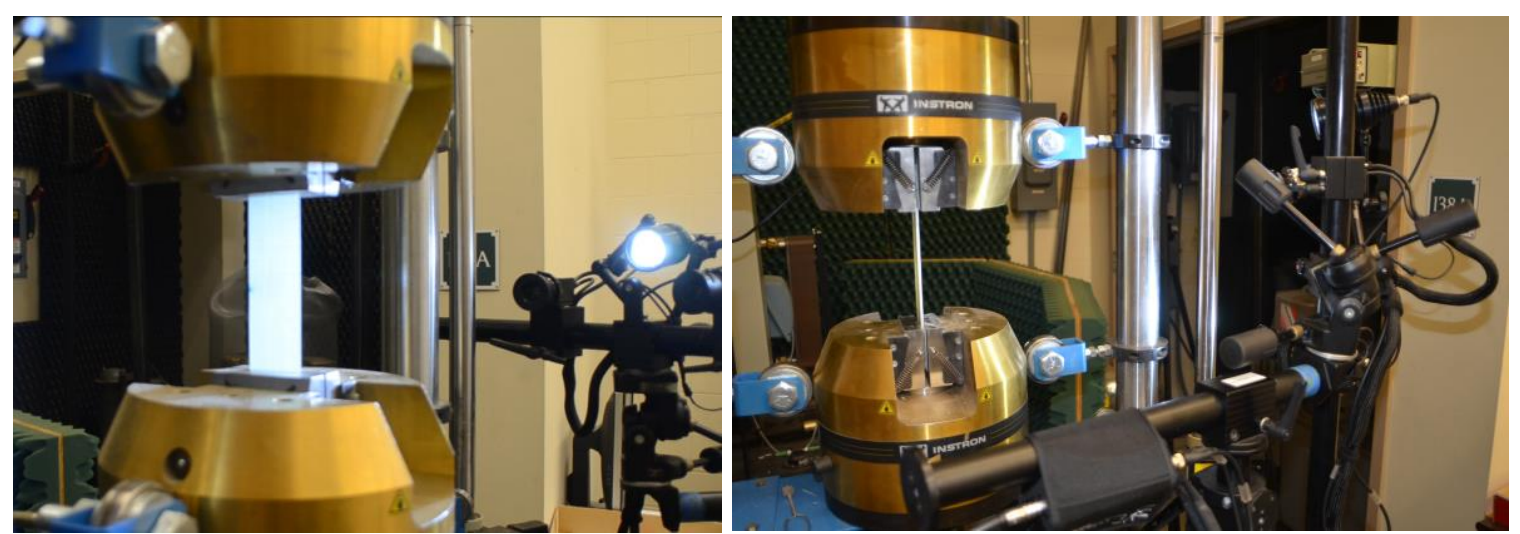

Figure 25: Test setup from first set of tests (left) and second set of tests (right). The Aramis cameras oriented horizontally for the first set and vertically for the second set.

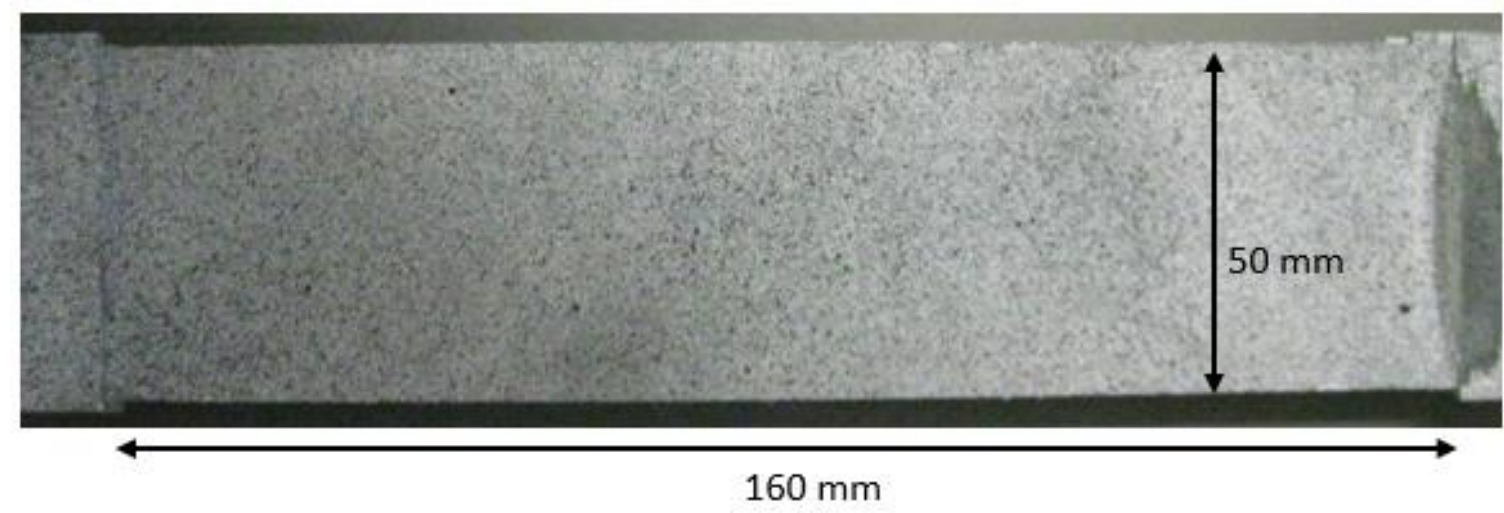

Figure 26: Typical speckle pattern painted on coupon for Aramis readings. Tabs can be seen on either end of coupon.

\section{Coupon Test Results}

Coupons from a variety of laminates were tested. Due to the new manufacturing method for the new CFIP test coupon, not all coupons and resulting data were usable. While 11 coupons were tested, only 6 have usable stress and strain data. This is because two of the coupons were not intended for strain data. These were painted for strain data with the DIC system but were used for direct imaging. Coupon data loss due to data corruption occurred with 2 coupons. Lastly, slipping in the grips and grip failure resulted in incomplete data for another coupon. Despite only 6 of the 11 coupons resulting in 
usable data, this is still enough for statistical relevance, therefore, more coupons were not made and tested.

The final data used for stress-strain plots and related calculations came from 4 different laminates. Three of these coupons are from a laminate made using a caul plate to reduce the out-of-plane portion of the IP wave. The impetus for reducing the out-ofplane portion is because coupons had been failing on the left side of the coupon. This was within the wave region down the length but in straight and slightly misaligned fiber tows. This was thought to be due to rotation in the grips caused by the change in thickness through the width. However, the coupons made with a caul plate resulted in minimal out-of-plane variation reduction. However, as discussed below in Chapter 4, a modeling investigation found that the out-of-plane thickness variation was found to not be the cause of larger strains on the left side. This was further investigated by testing two coupons back-to-back, aligned so that the thicker part of one coupon was aligned with the thinner part of the other coupon. While the neutral axis across the cross-section was slightly angled, the thickness that the grips felt overall similar across the width and down the length. This would greatly reduce rotation if this were present. The strain field was investigated and this still exhibited the left side tows straining more than right. After the modeling and experimental investigations, the cause is now thought to be due to the load distribution caused by the wave, not thickness distribution or rotation.

Images of visual damage progression in a typical CFIP coupon are shown in Figure 27. These images were taken with the Aramis cameras on coupons not painted for displacement measurements. The wave in these images is not centered but is towards the 
top of each image. The wave is roughly outlined with grey lines overlaying the images. The middle of the wave is marked down the length with a red arrow in each image. The wave location can also be seen as the damage accumulates around it until failure occurs. Damage progresses from Figure 27 (a) to Figure 27 (f). It can be seen that matrix cracks accumulate around the wave, primarily in the middle of the coupon across the width. This is because tows closer to the edges have smaller misalignment angles which results in lower shear stresses in the matrix. The tows on the left side are seen to fail first, followed by a cascading failure across the width which is considered to be total failure.

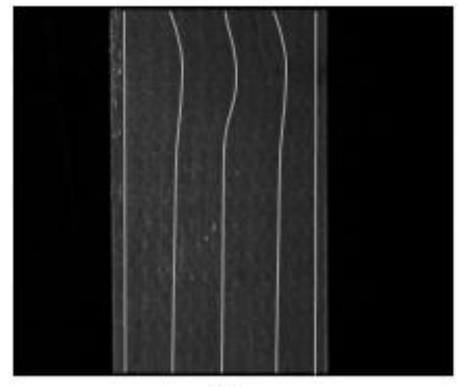

a

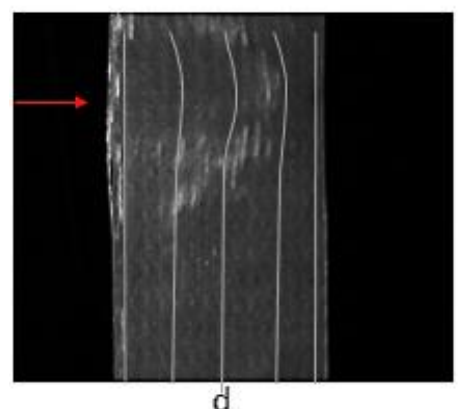

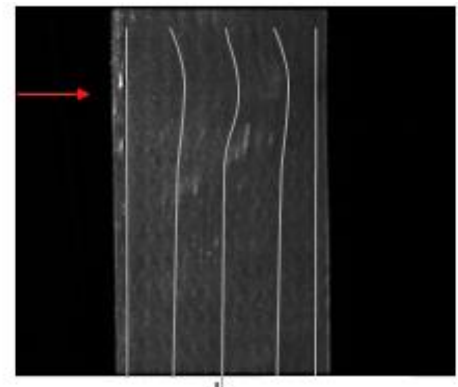

b

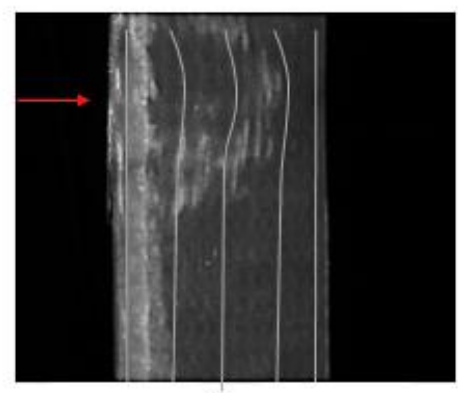

e

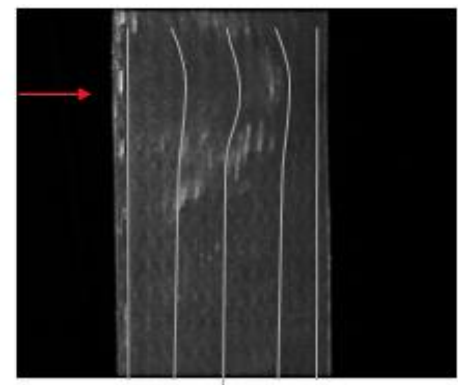

C

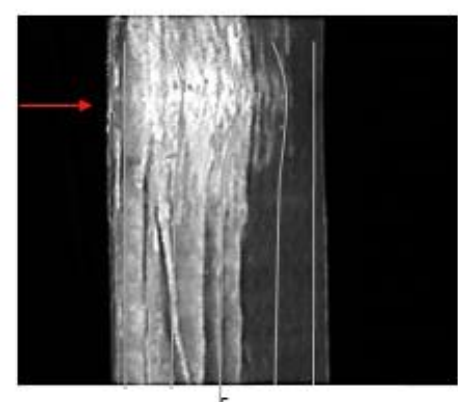

$f$

Figure 27: Images of a CFIP wave coupon during testing from (a) to (f). Grey lines overlaying coupon show fiber curvature and the arrow indicates the middle of the wave.

Left side tows (when the wave is orientated in this way, which is kept consistent throughout this work for discussion purposes) failing and then cascading across the width is the most common mode of failure seen. However, some coupons had failure initiate in 
the middle of the wave itself rather than on the side. Regardless of where failure

originates, the strain fields before failure appear very similar between coupons. A typical strain field for a CFIP coupon just before failure, as calculated using the Aramis DIC, is shown in Figure 28. Black lines roughly outline the shape of the wave.
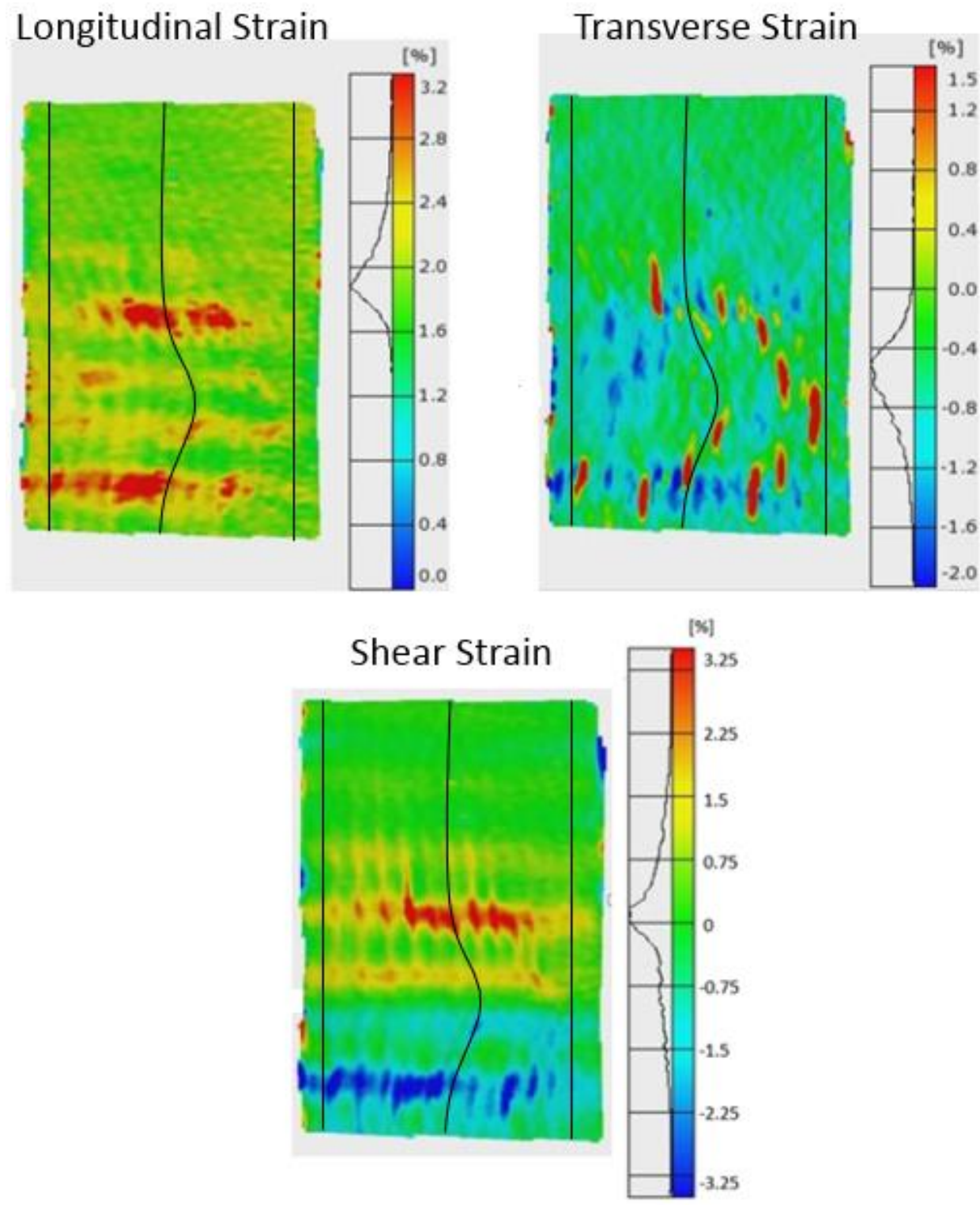

Figure 28: Typical strain distributions in CFIP wave coupon just before failure as captured with DIC. Longitudinal, transverse, and shear strains are top left, top right, and bottom respectively. Black lines outline the fiber tows in specific locations to show the location of the wave which is located slightly below the middle of the coupon. 
The longitudinal strain clearly shows two large bands of high strain on either side of the wave and two smaller bands inside those. These bands have been seen before and have been a cause of confusion. They appear to align with the maximum curvature of the fiber tows. These are discussed in more detail in Chapter 4. The transverse strain image above shows positive and negative strains both following a c-shape but mirrored from one another. The positive strains align with the locations of matrix cracking seen in Figure 27. The negative strain locations follow a mirrored curvature. Due to the spatial correlation between matrix cracking and transverse strain, it would seem that the matrix cracks have a large, positive transverse strain dependence. There is no correlation between matrix cracking and negative transverse strain distribution. This is discussed further below. In contrast to the previous waves which were uniform across the width, it can be seen that the strain distribution varies across the width. This can be seem most clearly in the transverse strain field and is due to the wave gradient across the width.

The shear strain image shows bands similar to the longitudinal strain field. However, these are divided so that one side of the wave has positive shear strain and the other side is negative. Also, the bands in the shear strain field are more centered around the middle across the width whereas the longitudinal strain is slightly higher towards the left side of the coupon. This is because the shear strain increases with misalignment angle, which is more centered about the middle. Due to the direct dependence on misalignment angle expected, only 1 band of high shear strain on either side of the wave would be expected. This is discussed in more detail below. 
The stresses for these coupons are calculated by dividing the load by the far-field cross-sectional area which was measured before testing. While the cross-sectional area distribution changes through the wave section, the total area is still assumed to be constant at each cross-section down the length. The strains are a bit more involved to calculate. Previous wave testing with MSUCG used a larger measuring volume for the DIC system. This means that more of the coupon was viewed by the DIC cameras. This allowed for full-field strain to be calculated. However, due to difficulties with calibration as well as a desire for better resolution, a smaller measuring volume was used for the testing done for this research. This means that the strain must be calculated slightly differently because the full-field was not captured. The testing for this research was performed in two rounds. Both rounds used an 80x65 mm measuring volume which means that an area $80 \times 65 \mathrm{~mm}$ can be viewed by the cameras. The first round had the 65 $\mathrm{mm}$ dimension aligned with the length of the coupons. The second round rotated the cameras so that the $80 \mathrm{~mm}$ dimension was aligned with the length so that more of the coupon could be measured. The strain was calculated by dividing the displacement by the length over which it was measured. For consistency, the length was the same for all coupons and the wave was centered in this length for each. The largest length that could be used for all coupons while keeping the wave in the center was found to be $45 \mathrm{~mm}$. This is smaller than $65 \mathrm{~mm}$ because the coupon moves slightly within the view of the cameras due to the displacement during testing. Also, the wave in the coupon is not always in the same starting location relative to the view of the camera between tests. The wavelength of the waves was about $50 \mathrm{~mm}$ meaning most of the wave is captured in the 
strain calculating area. Note that the cameras were poorly aligned for Coupon 6 so that only half of the wave is caught. Rather than not use this data, symmetry is assumed and the strain over half of the wave is used. Symmetry was checked and, for this coupon, is considered valid. The symmetry assumption is discussed in more detail below with the progressive failure mechanics. A schematic of this coupon showing the length over which strain is calculated can be seen in Figure 29 (left).

The strain value for the coupons depends on how it is calculated. The part of the coupon that will displace the most and, therefore, have the largest strain, is the wave portion. Measuring the strain over just the wave portion will result in a larger strain than if strain were calculated over the whole length. This must be kept in mind when comparing strains between these CFIP coupons and previously tested DFIP coupons. The strains are calculated over different areas as mentioned here and discussed below. The DFIP strain is over the whole $100 \mathrm{~mm}$ coupon length, as can be seen in the schematic in Figure 29 (right). The method used for calculating strain for the CFIP coupons tested herein is over the wave which results in larger strains than the full-field method used for the DFIP coupons from previous testing. 


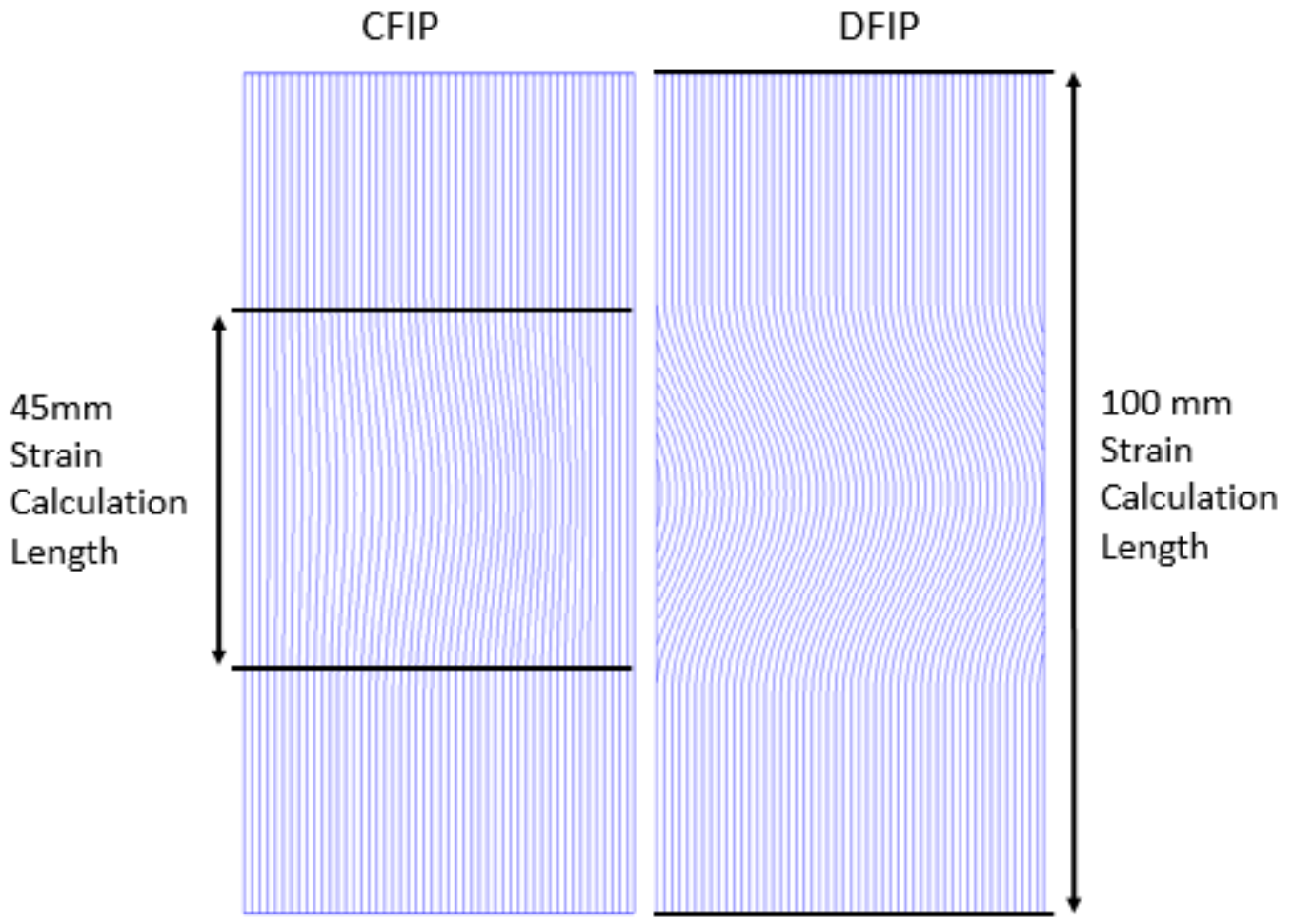

Figure 29: Coupon schematics from Figure 15 above, showing lengths over which strain is calculated. Strain over $45 \mathrm{~mm}$ wave for CFIP (left) and strain over whole $100 \mathrm{~mm}$ coupon length for DFIP (right).

The stress-strain curves for six coupons are shown in Figure 30. The variation between curves is likely due to variation between wave parameters between coupons. While plotting the maximum stress or strain against a wave parameter, such as misalignment angle, would give good insight into the effect of the wave severity, the wave parameters were not recorded with enough accuracy to make a meaningful plot. This inaccuracy is due to the maximum misalignment angles tested being within a 6 degree range and the variability in measuring the waves does not result in enough resolution for comparisons to be made. This is a small range of angles so rather than look at individual angles, they are all considered representative of this 4 to 10 degree range. 
Another reason for the variation in responses could be due to the fiber angles outside the maximum misaligned fiber. If there are more highly misaligned fiber tows near the maximum misaligned fiber tow, the effect of the wave can be expected to cause larger degradations than if the majority of the fibers are less misaligned. Using only maximum misalignment as the wave characterizing parameter as performed herein, both of these wave possibilities would be considered to have the same wave.

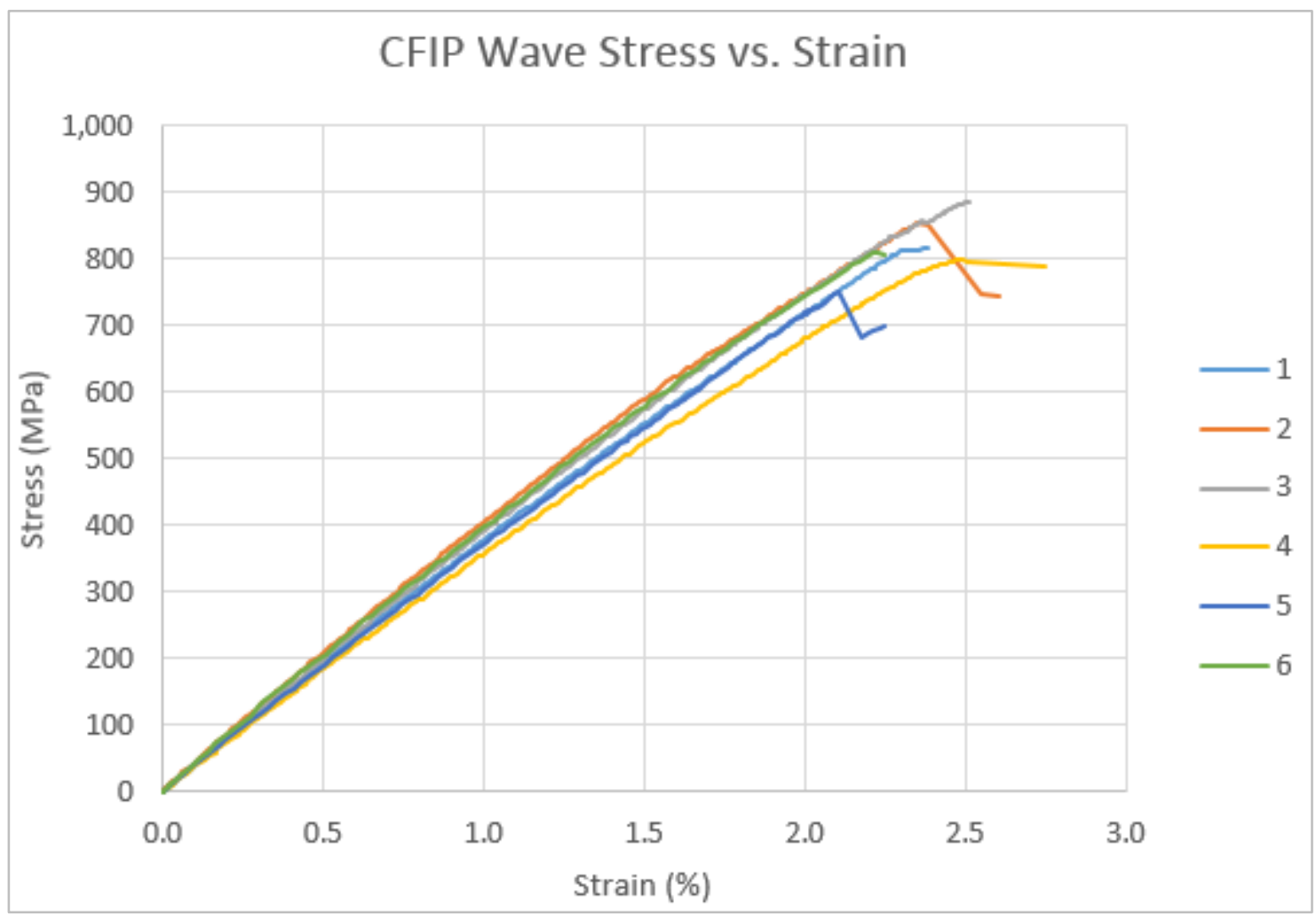

Figure 30: Stress-strain curves for CFIP wave coupons.

Quantitative comparisons are made with ultimate stress, strain at failure, and elastic moduli. The ultimate stress is taken to be the highest stress level reached. Strain at failure is the strain at ultimate stress. The elastic modulus for each of these coupons is calculated as the slope of the least squares best fit line between 0.1 and $0.4 \%$ strain. 
Ultimate stresses are between 760 and $890 \mathrm{MPa}$ with an average of $820 \mathrm{MPa}$. Strains at failure are between 2.1 and $2.5 \%$ with an average of $2.34 \%$. Elastic moduli are between 36.7 and 41.3 MPa with an average of 39.2 GPa. This general curve shape and these values are compared to DFIP coupons below.

\section{Coupon Test Discussion}

\section{$\underline{\text { Progressive Failure Mechanics }}$}

The two types of failures observed initiate either at the left side tows or near the maximum misalignment angle in the wave. As the coupon is loaded, the misaligned fiber tows displace horizontally in the opposite direction of the perturbing of the fibers in the wave. The degree of unrestrained displacement is proportional to the degree of misalignment. This is because the fiber tows straighten in order to support more load which is possible when less misaligned. Due to the misalignment gradient across the width, the less misaligned fibers restrict the shift and straightening of the more misaligned fibers. This restriction is caused by straightening of misaligned fibers pushing out less wavy fibers on the left side and pulling in less wavy fibers on the right side. This results in compressive strains on the left side of the coupon across the maximum amplitude and tensile strains on the right side, as seen in the DIC transverse strain data above in Figure 28. The resulting horizontal displacement field just before final failure, as captured with the DIC, can be seen in Figure 31. As expected, the largest horizontal displacement is in the middle of the coupon down the length due to this being the location of maximum misalignment and wave amplitude, thus requiring the largest displacement to return to unperturbed position. A strain concentration is caused in the 
left side tows from longitudinal straining rather than straightening, as well as added compressive transverse strain from the straightening of misaligned fibers. This can lead to failure in the left side straight fiber tows.

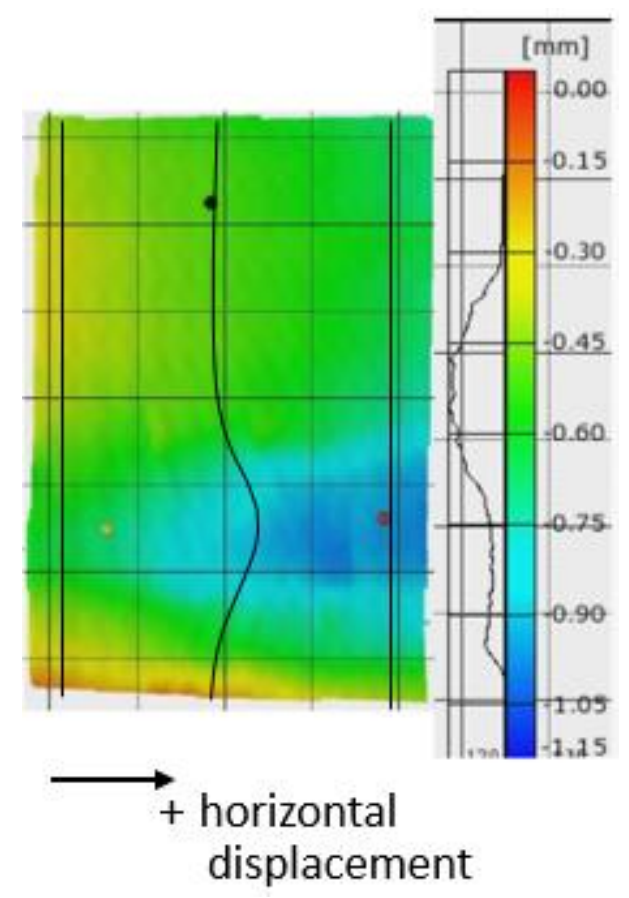

Figure 31: DIC image of a CFIP coupon showing horizontal displacement just before failure. Black lines outline the fiber tows in specific locations to show the location of the wave which is located slightly below the middle of the coupon.

Another type of failure observed was failure initiation in the wave. It is likely that the failure in the wave occurs with higher porosity. Failure in the wave is caused by shear stresses in the resin, due to fiber misalignment, causing crack initiation and propagation. The energy required for crack propagation depends on the crack surface which would be smaller with higher porosity. Future testing is required to determine this. 
As mentioned above, symmetry was assumed for Coupon 6 . The validity of this is now discussed. The half-wave longitudinal strains were calculated over the areas as shown in the schematic of the wave region in Figure 32.

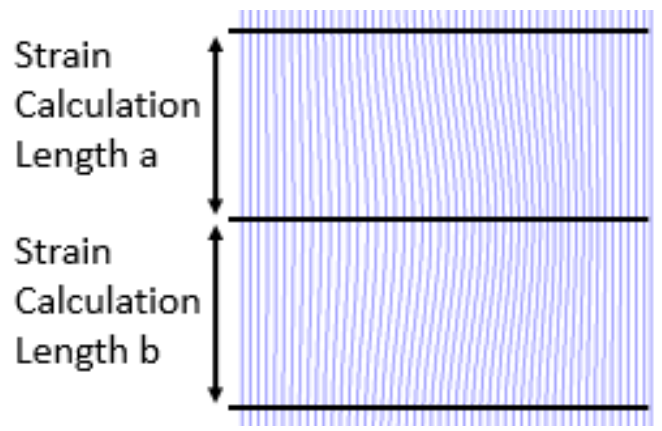

Figure 32: Strain regions for calculating half-wave symmetry shown on schematic of wave region.

It was found that the coupons with failure initiation in the left side tows all exhibited symmetry over the wave. Of the 3 coupons with failure initiating in the wave, 1 coupon had half-wave symmetry while the other 2 did not. The lack of symmetry is due to failure initiation being more localized and not symmetric over the wave. This causes one half of the wave to become more compliant while the other maintains stiffness. Due to Coupon 6 failing in the left side tows, half-wave symmetry is considered a valid assumption.

\section{DIC Shear Bands}

Initial shear strain is thought to be misalignment angle dependent. This being the case, large shear strains would be expected around the maximum misalignment angle and decrease in magnitude both across the width and down the length. This would cause 1 large shear strain band on either side of the wave. As resin damages, discretization and 
dispersion of maximum shear strain away from maximum misalignment angle may be expected, based on previous modeling. However, the DIC shear strain data did not capture this. Initial shear strains appeared in the same discrete bands as seen in the DIC images in Figure 28 above. This shows 2 discrete bands on either side of the wave rather than the 1 expected meaning that another factor is likely influencing these results. The shear bands can be seen outside the wave portion as well, although at much smaller magnitudes.

These discrete shear bands are also seen in control coupons. A DIC shear strain field of a control coupon with the same materials can be seen in Figure 33.

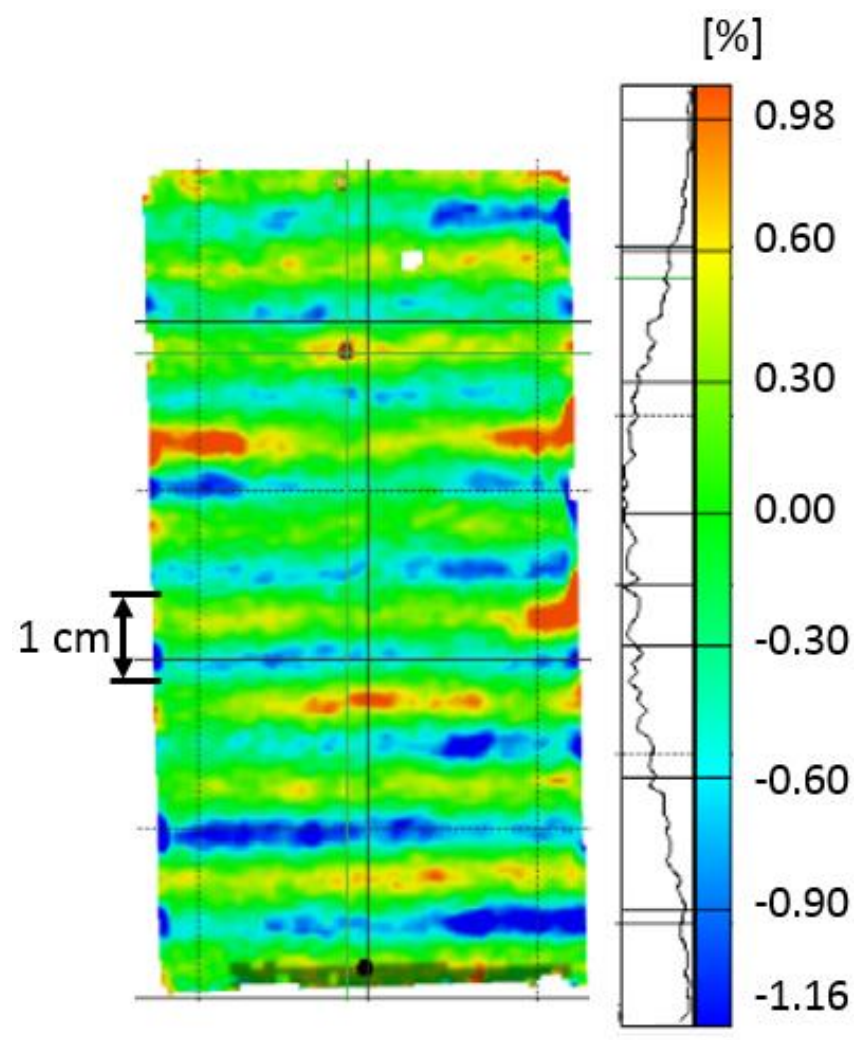

Figure 33: DIC shear strain distribution in control coupon. Pattern of alternating positive and negative shear band spacing labeled as $1 \mathrm{~cm}$. 
The pattern of alternating positive and negative shear bands is $1 \mathrm{~cm}$ long and repeats. The individual shear bands are separated by $0.5 \mathrm{~cm}$ from a maximum positive to maximum negative strain value. These shear bands in the control coupon may be caused by the stitching in the glass mats. The stitching is at a $+/-45$ degree angle to fiber direction which is in the $+/$ - shear direction. This can be seen in the image of the glass mat in Figure 34. The stitching pattern distance and spacing is the same as the distance between the strain bands in the shear strain field for the CFIP wave coupon. Both patterns are $1 \mathrm{~cm}$ long and repeat.

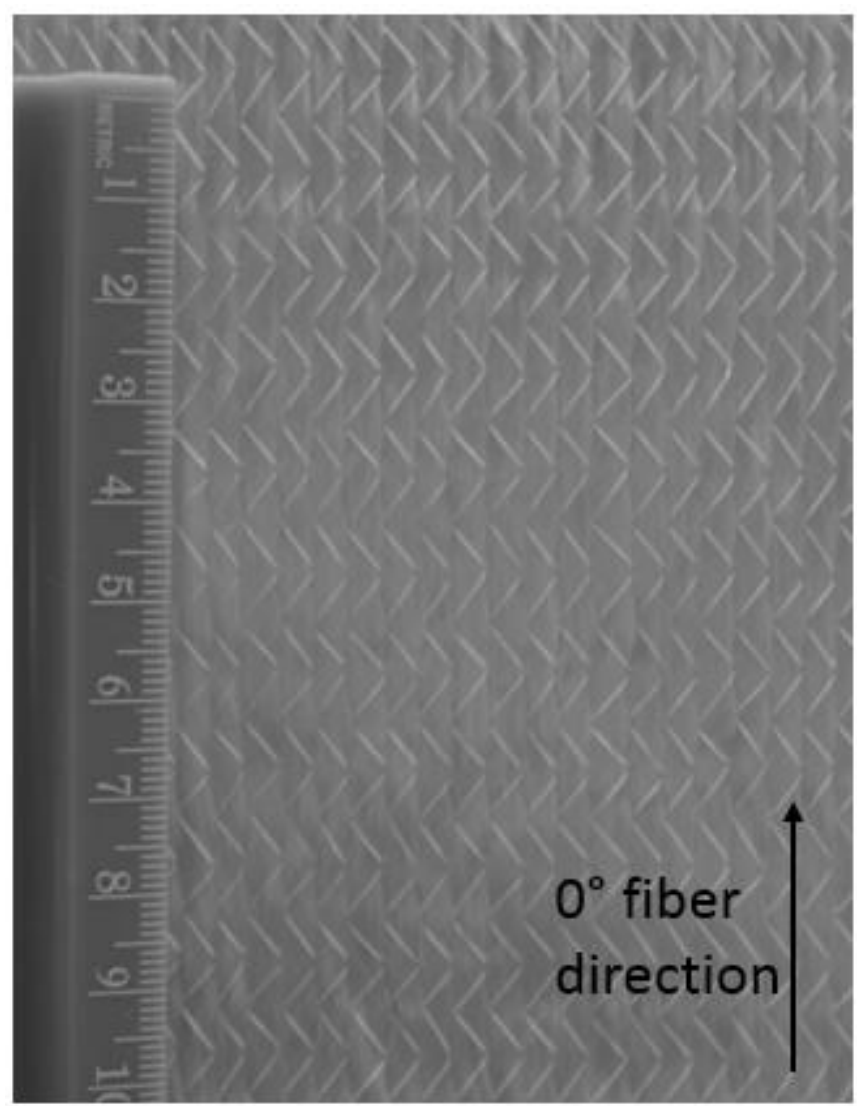

Figure 34: Glass mat showing angled stitching separating fiber bundles. Scale is in centimeters. Pattern is repeated down the length every $1 \mathrm{~cm}$. 
The shear bands in the CFIP wave coupon and control coupon, from Figure 28 and Figure 33 respectively, are compared in Figure 35. The strain scale has been set equal to that of the wave coupon. The dimensions are scaled so that the lengths are equal to investigate shear band spacing down the length. Both coupons were $5 \mathrm{~cm}$ wide but appear to be different widths here due to the angle of the Aramis cameras.

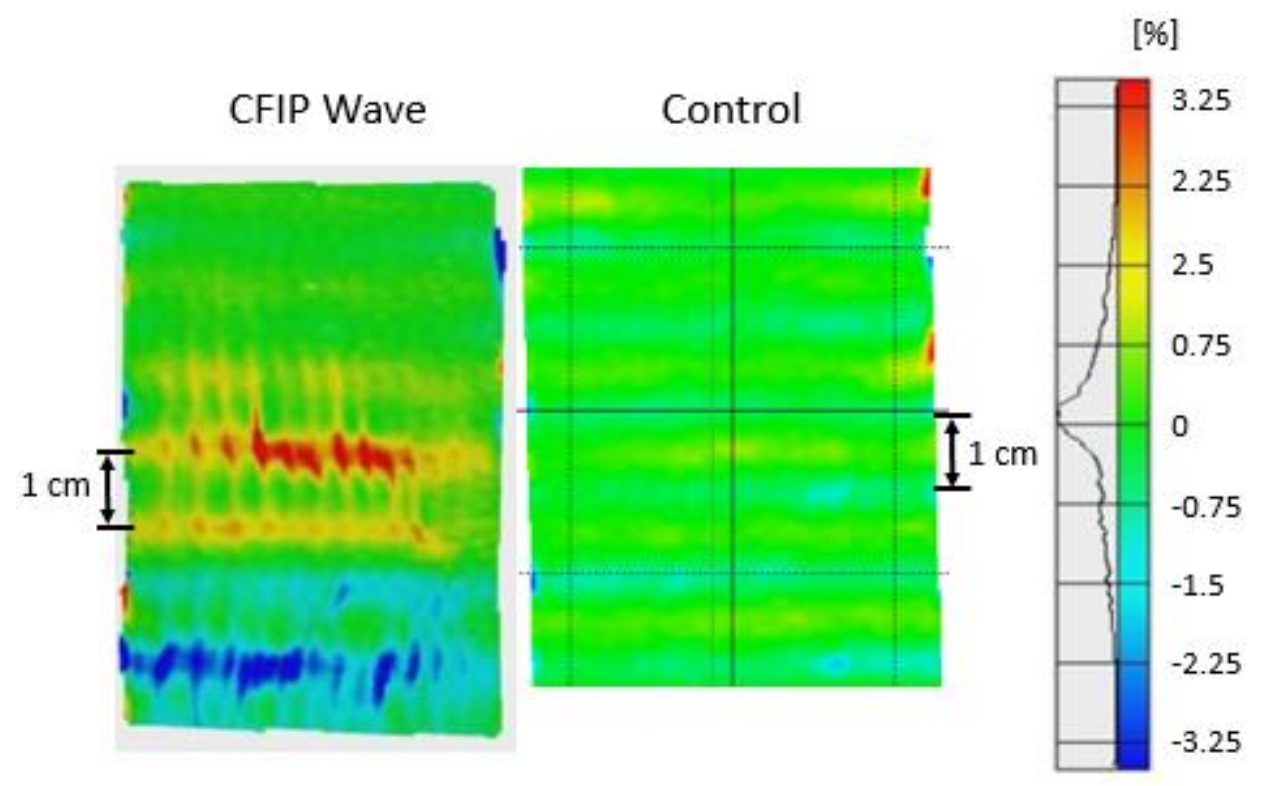

Figure 35: CFIP wave and control coupons shear strain fields. Shear band spacing can be seen to be the same between the two coupons.

It can be seen that the shear bands have the same spacing in both the wave and control coupons. This is notable because, despite the large influence of the wave on the magnitude, the spacing is the same with or without the wave. Due to both sets of coupons using glass mats with the same stitching pattern, shear band spacing similarity is expected if the stitching is the cause of the shear bands, which appears to be the case. 
The stitching is a polyester thread which, compared to the glass fibers, would be expected to have a negligible load carrying capability. A brief discussion is now included for how a polyester thread may cause the shear bands observed here.

The first possibility is that the shear bands are not indicative of the internal strain state and that these are just artifacts due to the measuring system. The DIC system measures surface strains and due to the stitching being on the measuring surface, these could cause the surface shear strain to appear much larger than the internal strain.

The second possibility is that the stitching actually creates very small in-plane waves in the fiber bundles that follow the pattern spacing of the stitching. These stitching induced waves have small misalignment angles which extend over very small wavelengths and have been previously overlooked or ignored due to their size. Looking at the CT scan of a single ply from Figure 23 (left) above, it can be seen that the fiber bundles do not follow straight lines. The fiber bundles follow small amplitude quasi-sine curves with a period of $1 \mathrm{~cm}$, equal to the stitching spacing. These small, stitchinginduced waves, however, do not appear to repeat consistently across the width and down the length. Due to the shear bands appearing more periodic and defined than these waves appear, these small waves may not be the cause of the shear bands. Similar to the previous possibility, if this is the case, the shear distribution measured by the DIC is likely not constant through the thickness because the stitching is likely not aligned and there is interference between waves of each ply.

If the stitching is the cause of the shear bands, as a result of either possibility discussed here, the DIC shear data is not indicative of the internal shear stress state. 
Further investigation into the causes of the shear bands is recommended as future work to determine the validity of the DIC shear strain results. Investigation into possible discrepancies in magnitude is also recommended. If the stitching causes constructive and destructive interference with the internal strain state, this may result in artifacts in magnitude as well as location.

Although the DIC shear strain data warrants future investigation, it is used for correlation with the model. The possibility of inaccuracies is kept in mind.

\section{$\underline{\text { CFIP and Control Comparison }}$}

Control and CFIP coupons are now compared. A stress-strain plot of a typical control coupon from BMT testing and a typical curve for a CFIP coupon are shown in Figure 36. The CFIP coupon is chosen to be Coupon 2. The strain measuring discrepancy for comparing CFIP and DFIP waves is considered negligible for comparing to control because the longitudinal strain over the control coupon is assumed constant down the length. The curves have very similar stiffness until just after $1.5 \%$ strain when the CFIP coupon becomes slightly less stiff until failure which occurs at a lower ultimate stress and strain at failure. 


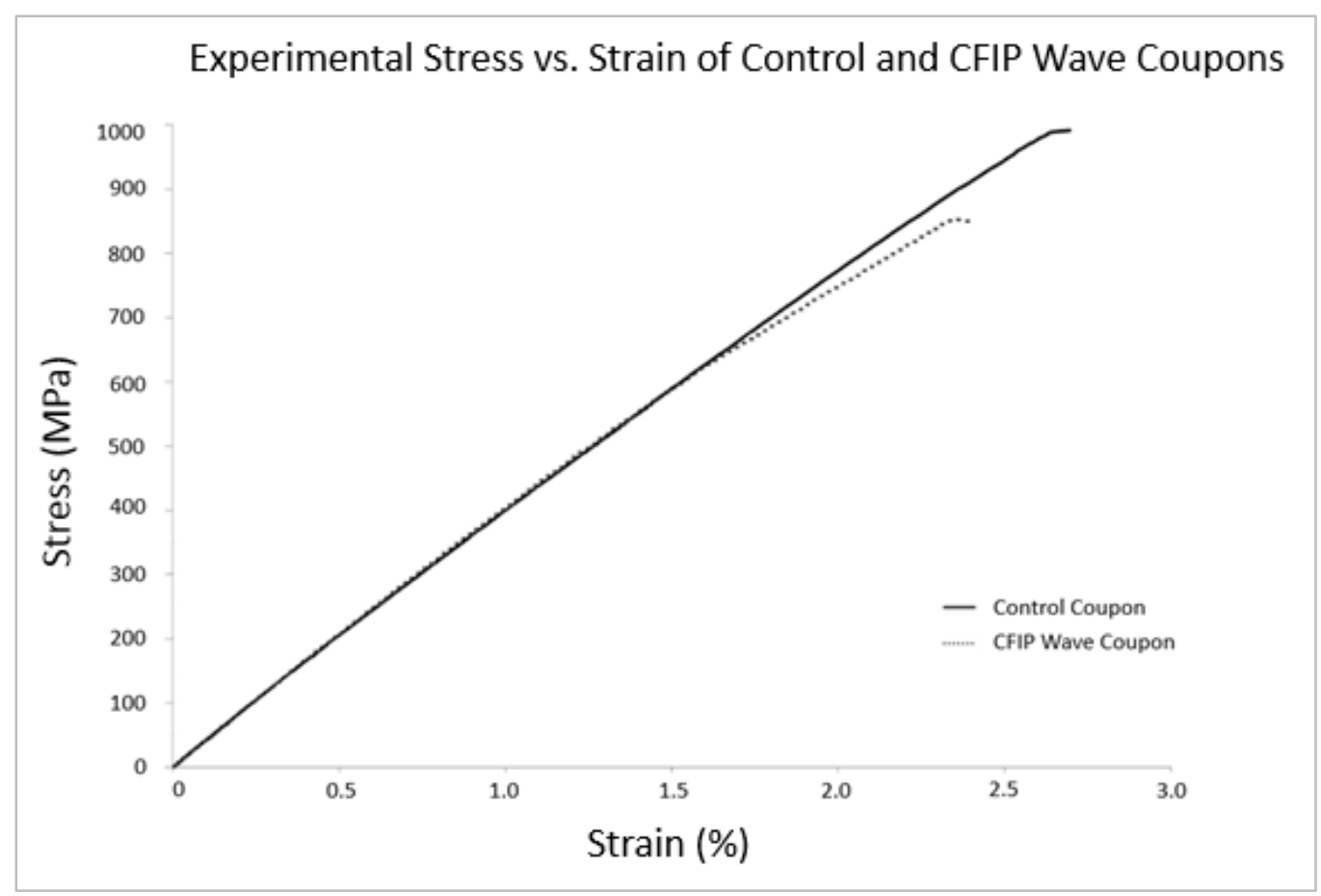

Figure 36: Typical stress-strain curves for control and CFIP wave coupons.

Average ultimate stress, average strain at failure, and average elastic modulus values are shown in Table 2 for CFIP coupons. Control values from above are also included for comparison.

Table 2: Static properties for control and CFIP wave coupons with standard deviation where available and percent of control for comparison.

\begin{tabular}{|c|c|c|}
\hline & Control & CFIP \\
\hline Ultimate Stress (MPa) & 990 & 820 \\
\hline Standard Dev & 40 & 43 \\
\hline$\%$ control & -- & 83 \\
\hline Strain at Failure (\%) & 2.6 & 2.34 \\
\hline Standard Dev & -- & 0.14 \\
\hline$\%$ control & -- & 90 \\
\hline Modulus of Elasticity (GPa) & 40.6 & 39.2 \\
\hline Standard Dev & -- & 1.6 \\
\hline$\%$ control & -- & 97 \\
\hline
\end{tabular}


The decrease in properties for the CFIP coupon compared to control is not as drastic as seen when comparing DFIP to control in Nelson, 2013, as discussed below. The ultimate stress is $83 \%$ of control, strain at failure is $90 \%$ of control, and elastic modulus is $97 \%$ of control. The average ultimate stress is $820 \pm 68 \mathrm{MPa}$. Also note that the standard deviation for CFIP ultimate stress is $43 \mathrm{MPa}$ compared to $40 \mathrm{MPa}$ for control. This is very similar despite the variability caused by the complex manufacturing method of the CFIP coupons compared to control coupons. The fiber volume fraction of CFIP coupons is considered the same as DFIP and control coupons made with the same materials and using the same manufacturing process. This fiber volume fraction is $55 \%$ (Riddle, 2013).

Due to the small variability seen in CFIP results, it can be assumed that the exact wave parameters do not have a large effect within the range of 4 to 10 degrees tested here. Considering that the outer tows are the same for all CFIP coupons, regardless of the wave severity, wave severity likely has less of an effect on response than it would in a DFIP coupon. More testing with varying wave sizes is suggested to further validate this. As mentioned above, larger misalignment angles with the current CFIP wave coupon cannot be made without increasing the out-of-plane variance to a degree which is deemed to no longer be negligible. Investigating larger misalignment angles of the CFIP wave type will require wider test specimens such as the sandwich beam discussed in Chapter 5 .

\section{$\underline{\text { CFIP and DFIP Comparison }}$}

CFIP and DFIP wave coupons are now compared. For this comparison it must be kept in mind that, while it is desired to only compare the different types of waves, there 
are more variables here that must be considered. These two wave types have different wave sizes and calculate strains differently. The CFIP wave misalignment angles are between 4 and 10 degrees while the DFIP coupons tested were between 29 and 49 degrees. Also, strains for the CFIP coupons are calculated just over the wave while the strain for the DFIP coupons is calculated over the entire $100 \mathrm{~mm}$ gage section.

Before comparing CFIP and DFIP test results, the DFIP results are briefly discussed. A typical stress-strain curve for one of these coupons in tension is shown in Figure 37. A control stress-strain curve is also included for comparison. While a variety of waves were tested, this curve is typical for a wave with a 49 degree average misalignment angle. Initial response for all similar wave coupons were similar and peak stress and strain varied by $\pm 58 \mathrm{MPa}$ and $\pm 0.15 \%$ respectively (Nelson, 2013).

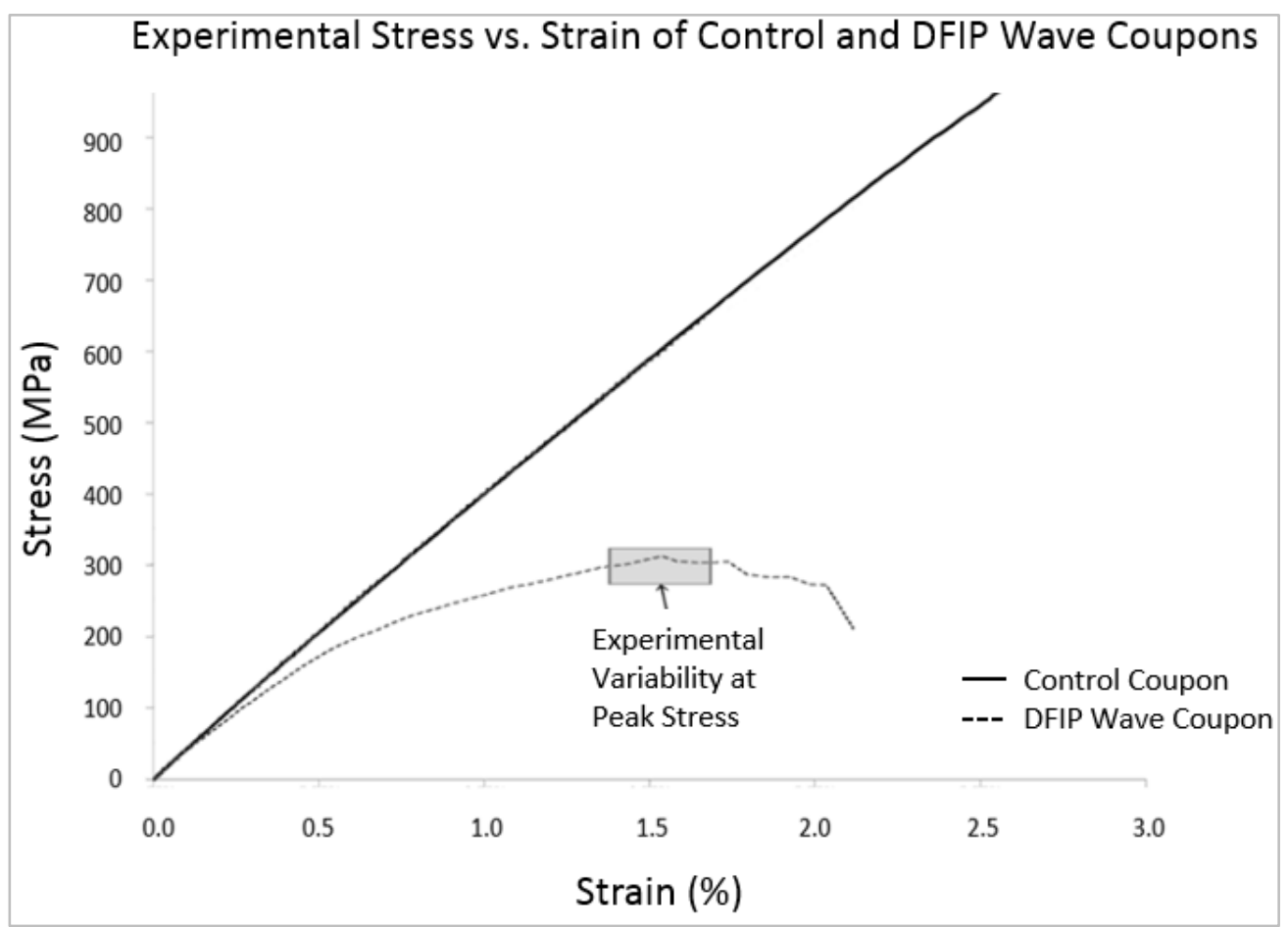

Figure 37: Typical stress-strain curves for control and discontinuous fiber IP (DFIP) wave coupons tested in tension (Nelson, 2013), (Riddle, 2013). 
Typical stress-strain curves for the CFIP and DFIP coupons with a typical control curve are shown above in Figure 36 and Figure 37, respectively. A stress-strain plot for CFIP and DFIP coupons is not included due to the number of variables, primarily the alternate methods for calculating strain. However, while strain values cannot be directly compared, stress and curve shape can be compared. It can be seen that the CFIP coupon is fairly stiff until failure while the DFIP coupon loses stiffness early on and exhibits softening over a large strain after ultimate stress has been reached. The early drop in stiffness in the DFIP coupon is due to the discontinuous fibers failing (Nelson, 2013). The CFIP coupons do not have these discontinuous fibers so this stiffness reduction does not occur. A lower strain at failure in DFIP coupons compared to CFIP is seen but this can be due to varying strain calculation areas as discussed above. The straight fibers strain less so strain over an area containing these will be smaller than just over the wave. Average values for a 29 degree DFIP wave coupon as well as the CFIP wave are shown in Table 3.

Table 3: Static properties for DFIP and CFIP wave coupons. DFIP values for wave with misalignment angle around 29 degrees. CFIP values for waves with misalignment angles between 4 and 10 degrees.

\begin{tabular}{|c|c|c|}
\hline & DFIP & CFIP \\
\hline Ultimate Stress (MPa) & 521 & 820 \\
\hline Standard Dev & 24 & 43 \\
\hline$\%$ control & 53 & 83 \\
\hline Strain at Failure (\%) & 1.66 & 2.34 \\
\hline Standard Dev & -- & 0.14 \\
\hline$\%$ control & 63 & 87 \\
\hline Modulus of Elasticity (GPa) & 39.6 & 39.2 \\
\hline Standard Dev & -- & 1.6 \\
\hline$\%$ control & 96 & 95 \\
\hline
\end{tabular}


From this table it can be seen that the ultimate stress and strain at failure is much higher in the CFIP coupons. This is a valid comparison for ultimate stress but, as mentioned above, not for strain at failure. A higher ultimate stress for the CFIP coupons is expected both because of wave type as well as smaller misalignment angles. The elastic moduli are very similar for both wave coupon types. The high percent of control for modulus of elasticity is thought to be due to the matrix locking fibers in place over the $0.1 \%$ to $0.4 \%$ strain which this was calculated. This means that the wave has only a small effect on initial stiffness (Nelson, 2013). This was observed because despite the misalignment angle for the CFIP coupons being smaller than that for the DFIP coupons, the elastic moduli knockdowns are about the same at $96 \%$ of control for DFIP and $95 \%$ for CFIP. It should also be noted that elastic moduli for DFIP and CFIP coupons are over different areas as well, due to the strain calculation differences mentioned above. This would lead the CFIP to have a lower value for elastic modulus but because these values are both so close to control, it appears that the wave does not have a large effect on initial elastic modulus.

Smaller degradations in material properties in CFIP coupons compared to DFIP is likely due to smaller misalignment angles as well as the straight fibers along the edges. The smaller misalignment angles are 4 to 10 degrees compared to 29 degrees. The latter reason further supports the hypothesis that current defect strength reductions based on previous DFIP coupon testing is too conservative for wind turbine blades. More research is required to differentiate the effects of wave severity and wave type. 
Lastly, the CFIP failure stress data is added to Figure 8 from above. This can be seen in Figure 38. The CFIP data partly overlaps with the DFIP data trendline but is mostly at a larger failure stress. Larger failure stress for CFIP data for a given misalignment angle is expected given the straight fibers. A misalignment angle in a DFIP coupon is the same throughout the width. In the CFIP coupon, however, this is the maximum angle reached which means that the misalignment angle of all but one tow is smaller than this angle.

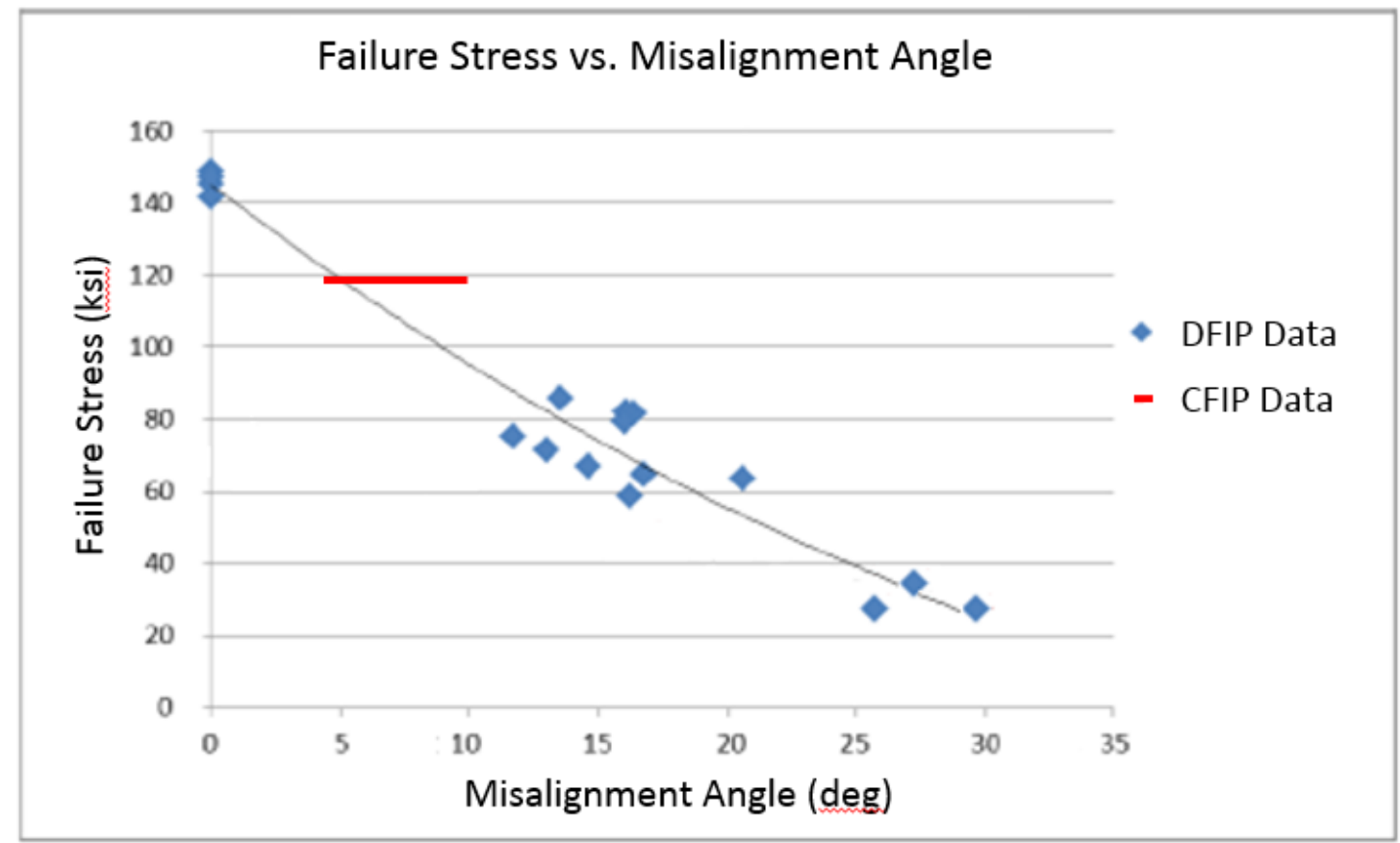

Figure 38: Failure Stress vs. Misalignment Angle for DFIP data and CFIP data. DFIP data as discrete points and CFIP data over range of misalignment angles.

\section{Coupon Testing Conclusions}

This chapter includes test impetus, methodology, and results for CFIP coupon testing. Qualitative and quantitative conclusions were drawn from these tests alone as well as through comparisons with control and DFIP coupons. 
It has been found that there is only a slight decrease in strain at failure and ultimate stress in the CFIP coupons compared to control in contrast to the DFIP coupons compared to control. This is due to both the wave type as well as the smaller wave severity of the CFIP coupons. Both CFIP and DFIP coupons have similar decreases in elastic modulus, which is slight. This is likely due to the matrix locking fibers in place before matrix cracking occurs, maintaining initial stiffness (Nelson, 2013). This theory of matrix locking is also further validated herein due to this holding true now for multiple wave types over multiple misalignment angles.

The stress-strain response of the CFIP coupon is much closer to control than the DFIP, which has a large softening portion not seen in the CFIP curve. Over this region, CFIP coupons strain $0.1 \%$ between ultimate stress and final failure, whereas DFIP coupons strain $0.5 \%$. This means that the CFIP wave failures are more sudden than DFIP, meaning failures of parts with CFIP waves will give less warning before catastrophic failure.

It is also notable that all CFIP stress-strain responses were similar within the range of 4 to 10 degree misalignment angles investigated. This is likely the result of less dependence on maximum misalignment angle due to the majority of the fibers being less misaligned than this angle.

The CFIP wave coupons had more complex strain fields than the DFIP coupons. Whereas the DFIP coupons were continuous across the width, the CFIP coupons vary. This caused strain distribution variation down the length in CFIP coupons, similar to 
DFIP, but also across the width. The added complexity likely needs another parameter to fully characterize the CFIP wave.

There is high spatial correlation between visual matrix cracking and positive transverse strain. Positive transverse strains are expected to have a larger effect than negative because transverse properties are matrix dominated and the resin is weaker in tension (Hexion, 2006). The correlation of transverse strain and visible matrix cracking is important to note because fiber waviness has been previously thought to cause shear dominated failures. The effect of both shear and transverse strains are investigated in Chapter 4.

Another important finding is the effect of the stitching in the glass mats on discretizing shear strain distribution down the length of the coupon. These test results were used to check for correlation with the FE model in Chapter 4 and the questionable accuracy must be kept in mind. Further research is recommended to verify whether stitching is the cause of the shear bands. This would include testing coupons made with glass mats with different stitching patterns or with the stitching removed. DIC imaging would be used to determine whether the shear bands have changed spacing to the new stitching pattern or cannot be seen if stitching has been removed. This research should also investigate if there is an effect on shear strain magnitude readings from the DIC.

Direct comparisons between CFIP and DFIP coupons could not be made due to the different misalignment angles. In order to make direct comparisons, the same misalignment angles of each wave type must be tested. CFIP coupons cannot be made with larger misalignment angles for reasons mentioned above, but DFIP coupons can be 
made with smaller misalignment angles. This is recommended as future work so that direct comparisons of the effect of the wave type can be made. 


\section{CHAPTER 4}

\section{COUPON MODELING}

\section{$\underline{\text { Motivation }}$}

Finite Element Modeling has a number of benefits. The first is verification of experimental tests. A lack of control in a test results in more variables, either known or unknown to the tester, which can lead to inaccurate conclusions. In modeling, however, all variables can easily be controlled. This allows for experimental validity as well as damage mechanisms involved to be investigated. This gives better insight into the mechanics of an experimental test in which the effect of altering different variables can be easily checked with multiple model runs with varying inputs, rather than continued testing. Another benefit of modeling is being able to predict the response of a part without testing. This is much faster and cheaper, however, development of an accurate predictive damage model is difficult. Creating an accurate, predictive progressive damage model for composite materials has been a goal of MSUCG. Large strides were made in the work of Nelson, 2013 and this is further developed herein.

\section{Combined Code Detailed Background and Updates}

The progressive damage model developed by MSUCG is discussed in more detail here. Details from the previous modeling iteration are discussed along with updates implemented herein. Damage in both models is considered any unrecoverable loss of energy such as crack creation and propagation. The same element types from the 
previous iteration of this modeling method are used herein. The fiber and resin together, modeled with the CDM approach, uses the CPS4 element. This is a 2D, 4-node, bilinear, continuum, plane stress element (Abaqus Analysis User's Manual v6.12, 2012). The resin between adjacent fiber bundles, modeled with the DDM approach, uses the COH2D4 element. This is a 2D, 4-node, cohesive element (Abaqus Analysis User's Manual v6.12, 2012). The previous iteration of this model employed $0.5 \mathrm{~mm}$ by $0.5 \mathrm{~mm}$ continuum elements to model a $100 \mathrm{~mm}$ long and $50 \mathrm{~mm}$ wide DFIP coupon. These were separated with zero thickness cohesive elements across the width of the coupon every 2 elements to model fiber tows $1 \mathrm{~mm}$ wide. The cohesive element columns extend the whole length of the coupon. All elements were set to have a thickness of $4 \mathrm{~mm}$.

The changes from the previous modeling iteration are new wave type, coupon length, cohesive element geometry, and cohesive element material properties. The new wave type is discussed below in the Mesh Generation section. These other three updates are now discussed.

The first change from the previous modeling iteration is that this model is now $160 \mathrm{~mm}$ long to model lengths similar to those of the coupons tested in this work. The second change is the cohesive element spacing. The cohesive elements are included to allow for discrete, local, matrix cracking. The previous model placed cohesive elements every $1 \mathrm{~mm}$ across the width, allowing for matrix damage and cracking to develop every $1 \mathrm{~mm}$. This was based on the fiber tow width of $1 \mathrm{~mm}$. However, looking at the fiber glass mat in Figure 34 above, which is included here with the width labeled in Figure 39, the visible spacing of the fibers appears to be $4 \mathrm{~mm}$. This $4 \mathrm{~mm}$ distance is the fiber 
bundle width, separated by the cross-stitching. Each bundle contains four $1 \mathrm{~mm}$ wide fiber tows. Once injected with resin, the resin-rich areas appear to be between $4 \mathrm{~mm}$ wide fiber bundles, not $1 \mathrm{~mm}$ fiber tows. This was determined through visual and CT scan investigation, as well as the discrete strain distributions in the DIC images.

Visually, the $4 \mathrm{~mm}$ resin-rich spacing can be seen above in Figure 14 and below Figure 43. With CT imaging, this can be seen in Figure 23 and Figure 24. This $4 \mathrm{~mm}$ spacing also appears to be consistent with the DIC data. The longitudinal strain field from Figure 28 above is included here in Figure 40 with two high strain region separations labeled to show $4 \mathrm{~mm}$ spacing. Therefore, rather than space the cohesive elements every $1 \mathrm{~mm}$ to discretely model fiber tow widths, the cohesive elements spacing is updated to a $4 \mathrm{~mm}$ to discretely model resin-rich areas between fiber bundle.

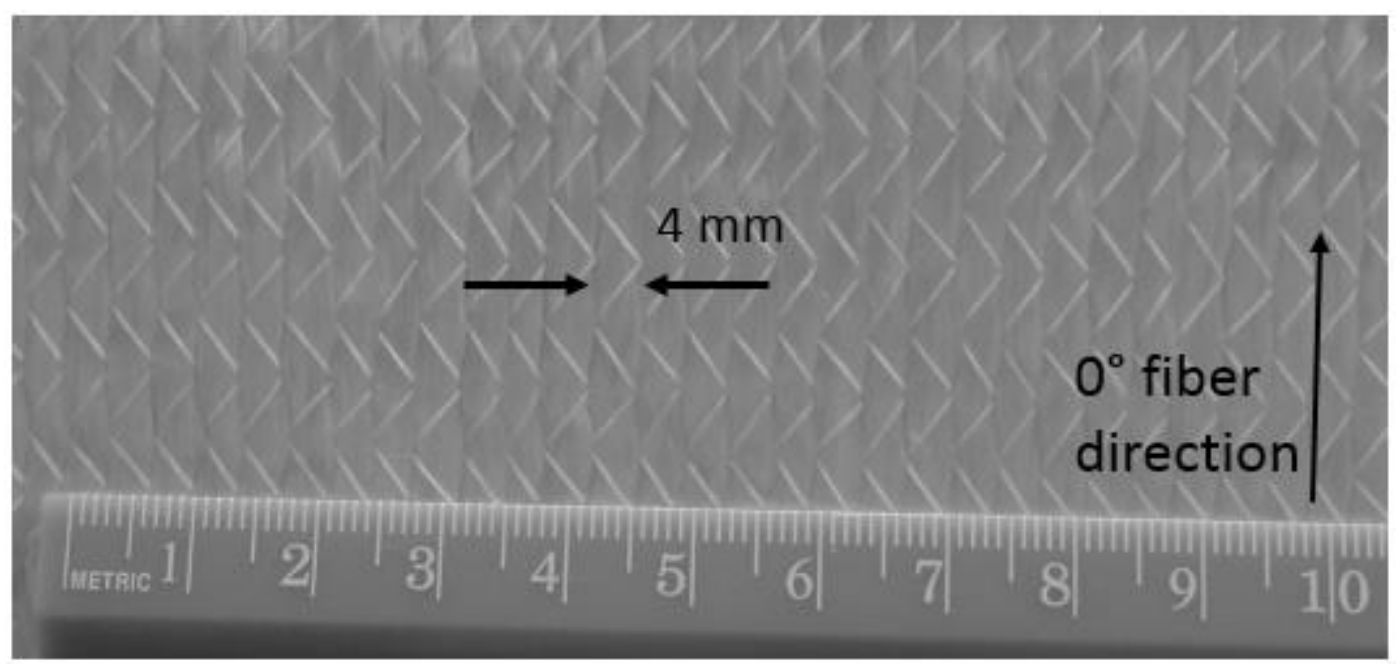

Figure 39: Glass fiber mat showing $4 \mathrm{~mm}$ width fiber bundles separated by stitching. 


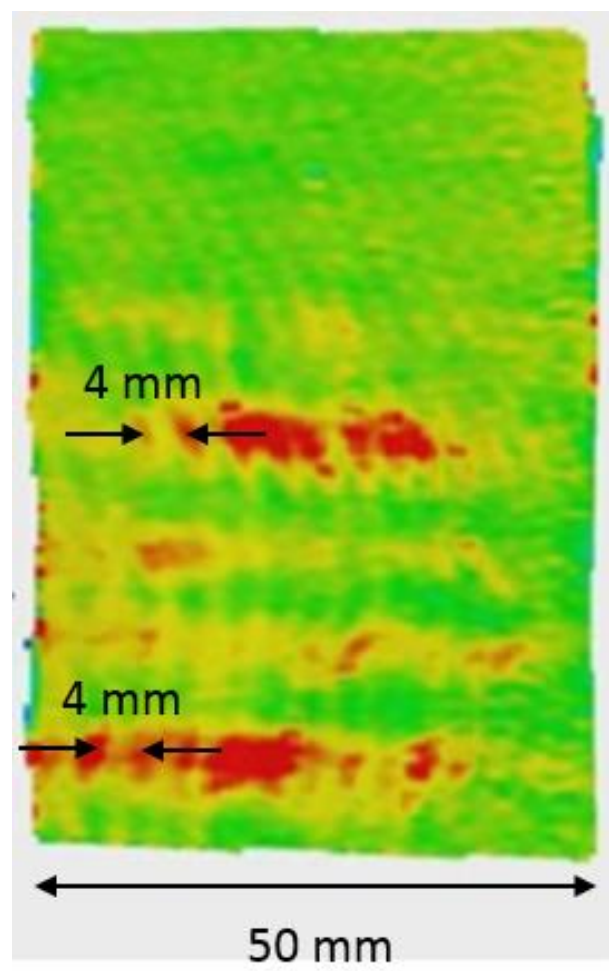

Figure 40: Experimental longitudinal strain field obtained with the DIC showing high strain regions separated by fiber bundle distance of $4 \mathrm{~mm}$.

The effect of changing the cohesive element separation was found to be negligible on the strain distributions but does affect the stress-strain response. Further, it was found that decreasing the number of cohesive elements decreases the processing time. These effects are discussed in more detail below.

Due to this new spacing, eight $0.5 \mathrm{~mm}$ wide continuum elements separated each cohesive element across the width, whereas before this was two $0.5 \mathrm{~mm}$. The mesh can be seen in Figure 41. This mesh is for an 8 degree maximum misalignment angle because this is the average of coupons investigated experimentally. Details on mesh generation are included below. 


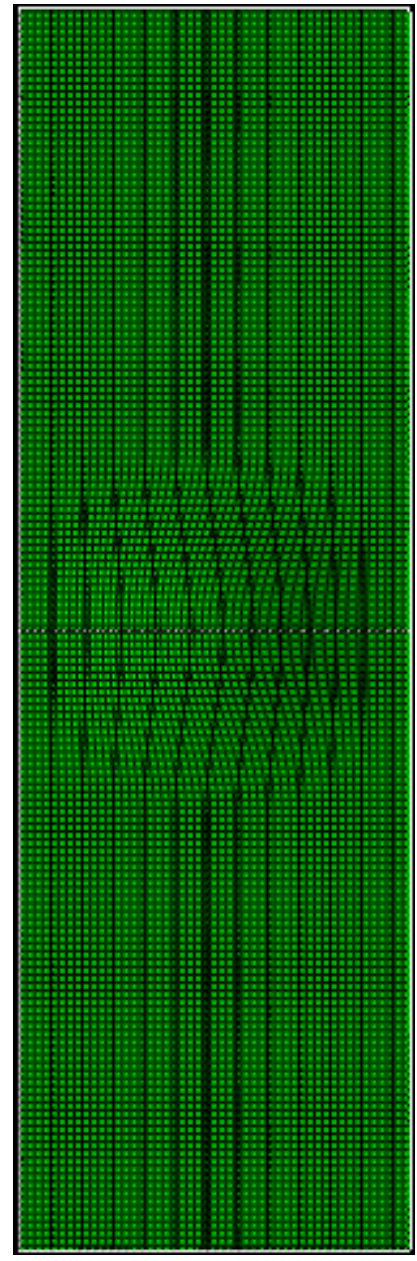

Figure 41: CFIP wave coupon model mesh. Cohesive columns can be seen as darker columns spaced every 8 continuum elements across the width.

The continuum element response is defined with a UMAT as discussed below.

The UMAT requires material property inputs as labeled in the code here:

*MATERIAL, NAME $=$ COMP

*USER MATERIAL, CONSTANTS $=20$

$E_{1}, E_{2}, v_{1}, G_{12}$,

Shear Strains 1-8,

Shear Stresses 1-8 
The first input is longitudinal modulus, second input is transverse modulus, third input is Poisson's ratio, and fourth input is shear modulus. These values are from previous testing and can be seen in Table 4.

Table 4: Material properties from previous testing (Nelson, 2013).

\begin{tabular}{|c|c|c|c|}
\hline $\mathbf{E}_{\mathbf{1}}(\mathbf{G P a})$ & $\mathbf{E}_{\mathbf{2}}(\mathbf{G P a})$ & $\mathbf{v}_{\mathbf{1}}(\mathbf{G P a})$ & $\mathbf{G}_{\mathbf{1 2}} \mathbf{( G P a )}$ \\
\hline 40.6 & 4.5 & 0.27 & 16.8 \\
\hline
\end{tabular}

The remaining inputs define the shear response. The fifth through twelfth inputs are shear strains, and thirteenth to twentieth are shear stresses. These define points on the shear stress-strain curve for this laminate as seen in Figure 9 above, from which secant modulus is updated. These values can be seen in Table 5. Although it would require slight changes to the UMAT, the number of constants defining the shear response can be changed depending on how many points is best suited to define the shear response for a given material. The points are chosen to be at locations in which there is a significant change in slope. These values are not changed from the previous work because these are the same materials and same infusion process (Nelson, 2013).

Table 5: Shear stress and strain values from testing to define nonlinear shear response (Nelson, 2013).

\begin{tabular}{|l|c|c|c|c|c|c|c|c|}
\hline State & $\mathbf{1}$ & $\mathbf{2}$ & $\mathbf{3}$ & $\mathbf{4}$ & $\mathbf{5}$ & $\mathbf{6}$ & $\mathbf{7}$ & $\mathbf{8}$ \\
\hline Strain & 0.002 & 0.003 & 0.0056 & 0.0091 & 0.0159 & 0.024 & 0.0356 & 0.0454 \\
\hline Stress (MPa) & 25 & 53 & 70 & 93 & 96 & 103 & 103 & 103 \\
\hline
\end{tabular}

The cohesive elements are defined using a bi-linear criterion as discussed above and shown in Figure 10. The required inputs for this response are damage initiation 
(*DAMAGE INITIATION), damage evolution (*DAMAGE EVOLUTION), and elastic stiffness $\left({ }^{*} E L A S T I C\right)$. The lines for cohesive material property definitions are:

*MATERIAL, NAME $=$ COHESIVE

*DAMAGE INITIATION, CRITERION=MAXS

$T_{\text {IMAX }}, T 2_{M A X}$

*DAMAGE EVOLUTION, TYPE $=E N E R G Y$, MIXED MODE BEHAVIOR $=B K$, $P O W E R=2.284$

$G_{I C}, G_{I I C}$

*ELASTIC, TYPE $=$ TRACTION

$K_{1 E F F}, K_{2 E F F}$

The *DAMAGE INITIATION keyword is used to determine the stress level at which the cohesive elements will begin to damage. The inputs are normal and shear stresses $\left(\mathrm{S}_{22}\right.$ and $\left.\mathrm{S}_{12}\right)$. These inputs are investigated and altered as discussed below, based on matching model and DIC data damage initiation. The input values for all keywords used in the previous iteration of model for the DFIP wave coupon can be seen in Table 6. These values were used as a starting point for this CFIP wave coupon model. The *DAMAGE EVOLUTION keyword determines the Mode I and Mode II critical energy release rates. These values are the energy under the curve for the bi-linear criterion in both modes. These inputs are resin material properties determined from Double-Cantilever Beam (DCB) and End-Notched Flexure (ENF) tests respectively. The values used here are from these tests which have been performed in Nelson, 2013 based on ASTM D5528-13 and ASTM WK22949 test standards. Since these material properties come from experimental testing, they are direct material properties. Tuning between model geometry should not be necessary for direct material properties. However, there are complications with the accuracy using the direct resin material properties caused by the inaccurate through-thickness uniformity assumption of the 
model. Basically, misalignment between plies, not directly captured in the model, results in increased crack length and damage to failure which will now be accounted for in the model. Therefore, these resin values will be changed from the previously developed code.

The last cohesive element property is the elastic moduli of the resin which uses the *ELASTIC keyword. Here it is used to set the uncoupled traction for cohesive elements. The exact inputs have been determined through parametric studies performed in previous work. These values were also investigated here and it was found that increasing or decreasing the elastic traction by an order of magnitude affects convergence. Up to the point of divergence however, the stress-strain response was the same for all three model runs with varying elastic traction inputs. The *ELASTIC inputs from previous parametric studies, included above, are used in the model herein.

The third change from the previous model iteration to that herein is to the *DAMAGE INITIATION and *DAMAGE EVOLUTION values. Details on the methods for updating the inputs for both of these keywords is included below. The third cohesive element property set, elastic moduli, is not changed. It should be noted that once cohesive element properties are determined, they will be the same for each cohesive element. This varies from the previous iteration which required weaker cohesive elements in specific locations to initiate failure similarly to that seen experimentally. This means that, due to the new wave type, this is a more predictive model and mesh complexity is reduced by implementing only identical cohesive elements. 
Table 6: Cohesive element material properties used in previous iteration of model for DFIP wave coupon.

\begin{tabular}{|c|c|c|c|c|c|}
\hline $\mathrm{T}_{1 \mathrm{MAX}}(\mathrm{MPa})$ & $\mathrm{T}_{2} \mathrm{MAX}(\mathrm{MPa})$ & $\mathrm{G}_{\mathrm{IC}}\left(\mathrm{J} / \mathrm{m}^{2}\right)$ & $\mathrm{G}_{\text {IIC }}\left(\mathrm{J} / \mathrm{m}^{2}\right)$ & $\mathrm{K}_{1 \mathrm{EFF}}(\mathrm{MPa})$ & $\mathrm{K}_{2 \mathrm{EFF}}(\mathrm{MPa})$ \\
\hline 110 & 110 & 0.806 & 1.524 & $2.2 \mathrm{E} 6$ & $2.2 \mathrm{E} 6$ \\
\hline
\end{tabular}

Failure criteria is now discussed. At the time of the development of this modeling method, Abaqus only allowed one subroutine at a time. Since this model uses a user material subroutine to define nonlinear shear softening, no other subroutines can be used. This means that a subroutine for failure of bulk elements cannot be included. Therefore, coupon failure must be determined by the user.

Failure for this model was based on full-field longitudinal strain, similar to the failure criteria in the previous work of Nelson, 2013. As discussed above, average strain at failure for the coupons was found to be $2.34 \%$ strain. This was strain over the wave, therefore, a longitudinal strain of $2.34 \%$ over the $50 \mathrm{~mm}$ long wave requires a displacement of $1.17 \mathrm{~mm}$. A wave displacement around this value was used to get a ballpark for failure based on wave region longitudinal strain. Exact determination of failure is discussed in more detail below.

\section{$\underline{\text { UMAT }}$}

The UMAT subroutine code has been previously developed and is used to capture the non-linear shear response of continuum elements in the model. Using a UMAT in Abaqus can be quite difficult although the theory itself is fairly simple. The subroutine is coded in FORTRAN. The basics of the UMAT are discussed here. For more details on the UMAT as well as the code in its entirety, refer to Nelson, 2013. 
The inputs for the UMAT are entered using the *USER MATERIAL keyword as discussed above. While many background calculations are involved in the UMAT, only the fundamental steps are discussed here. At each increment/step the shear stress is calculated for each element and checked against the shear stress-strain inputs. If the shear stress is within the linear portion, then the values are recalculated. If the shear stress is no longer within the linear portion then stresses at each integration point of the element are calculated and used to update the state variables accordingly. This calculates the secant modulus from the input shear stress-strain points. More information on the UMAT can be found in Nelson, 2013.

\section{$\underline{\text { Mesh Generation }}$}

The first step in generating the mesh is defining nodes in a 2D grid pattern of the coupon dimensions of $50 \mathrm{~mm}$ width and $160 \mathrm{~mm}$ height. The nodes have a separation of $0.5 \mathrm{~mm}$ in each direction to match desired element dimensions. The nodes within the wave area are then displaced to capture the wave geometry. A sine curve of the desired wave geometry is used to displace the middle node column to match the geometry of the most perturbed fiber tow in the coupon. This ensures that the maximum misalignment angle in the model matches those in the coupons tested. Due to the severity of misalignment varying across the width, this sine curve cannot be applied to all node columns. Therefore, a second sine curve is employed which varies from zero on either edge, to one in the middle of the coupon. The actual displacement of each node location is then multiplied by both sine curves to ensure that the maximum misaligned fiber bundle geometry is represented, while the edge fibers are unperturbed. 
Once the wave has been introduced to the nodes, the elements are defined based on the existing nodes. As implemented in previous iterations of this modeling method, in order to reduce processing times, only half of the coupon is modeled down the length and symmetry is assumed. This means that only the top half of the nodes are connected to form elements. The boundary conditions discussed below are implemented to allow for symmetry to be modeled.

The element material definitions must then be updated so that the material properties align with the wave rather than global direction. This is accomplished by defining the orientation for each element individually. The angle between the midpoint on the top and bottom of each element is calculated and used to define the orientation for its material properties. The material properties are kept orthogonal and rotated from global directions to this new orientation. This allows for material properties to match those of the glass fibers in the wave.

As discussed above, CFIP waves cause a slight out-of-plane portion in the coupon. This is accounted for in the model. Because this is a 2D model, constitutive thicknesses for all elements must be entered. By defining each element as its own element section, the thickness can be entered individually for each element. The thickness is determined by setting each element equal to an initial, unflawed volume size. The initial volume of each element is set to $1 \mathrm{~mm}^{3}$, which is base multiplied by height and thickness. For elements outside the wave region, the base and height are $0.5 \mathrm{~mm}$ and the thickness is $4 \mathrm{~mm}$. The elements in the wave have varying base and height so the unit volume is divided by this base-height area to calculate the thickness resulting in the 
normalized volume of $1 \mathrm{~mm}^{3}$. This calculated thickness for each element is entered for that element's thickness. The finished model with updated material properties was run with both thickness variation and without to investigate the effect of the out-of-plane portion. It was found that the slight thickness variation observed in experimental coupons had a negligible effect.

The nodes along the top edge and midline are defined to distinct node sets which are used in the boundary conditions to set displacement. The top edge node set has a vertical displacement boundary condition to input load displacement. This node set also has a zero displacement horizontal boundary condition. The midline node set has a zero displacement vertical boundary condition and is free in the horizontal.

This code is output by MATLAB as a .inp file. This file, along with the UMAT previously developed by MSUCG, is entered into Abaqus. The code includes node definitions, continuum element definitions, cohesive element definitions, material properties, boundary conditions, and solver definition.

\section{Cohesive Element Material Property Changes}

$\underline{\text { Impetus }}$

The cohesive element material properties used in the previously developed code are updated herein. These properties are defined with the $* D A M A G E$ INITIATION, *DAMAGE EVOLUTION, and *ELASTIC keywords. These three keywords define the bi-linear traction-separation criteria as can be seen in Figure 10 above which is included here again in Figure 42. Each cohesive element has Mode I (normal) and Mode II (shear) 
responses and therefore, separate bi-linear traction-separation criteria for each mode of loading.

The *DAMAGE INITIATION values are the normal and shear stress levels at which the cohesive elements are damaged, which corresponds to $T$ in Figure 42. Therefore, this should be a material property of the resin rather than a tunable input between wave types or wave severities. These stress levels were not determined through direct experimental testing, but were determined through a combination of analytical and experimental methods so that the damage initiates in the model at the same far-field strain as seen in testing. This results in lower *DAMAGE INITIATION values than those used in the DFIP wave model, as discussed below. Once the *DAMAGE INITIATION inputs have been decreased, through-thickness failures occur in the model at much lower fullfield strains than seen in testing. This means that the *DAMAGE EVOLUTION inputs must also be updated because these inputs are the energy released which is $G_{c}$, the area under the curve, in Figure 42. These inputs are also a resin-dominated material property with geometry dependence as discussed below. 


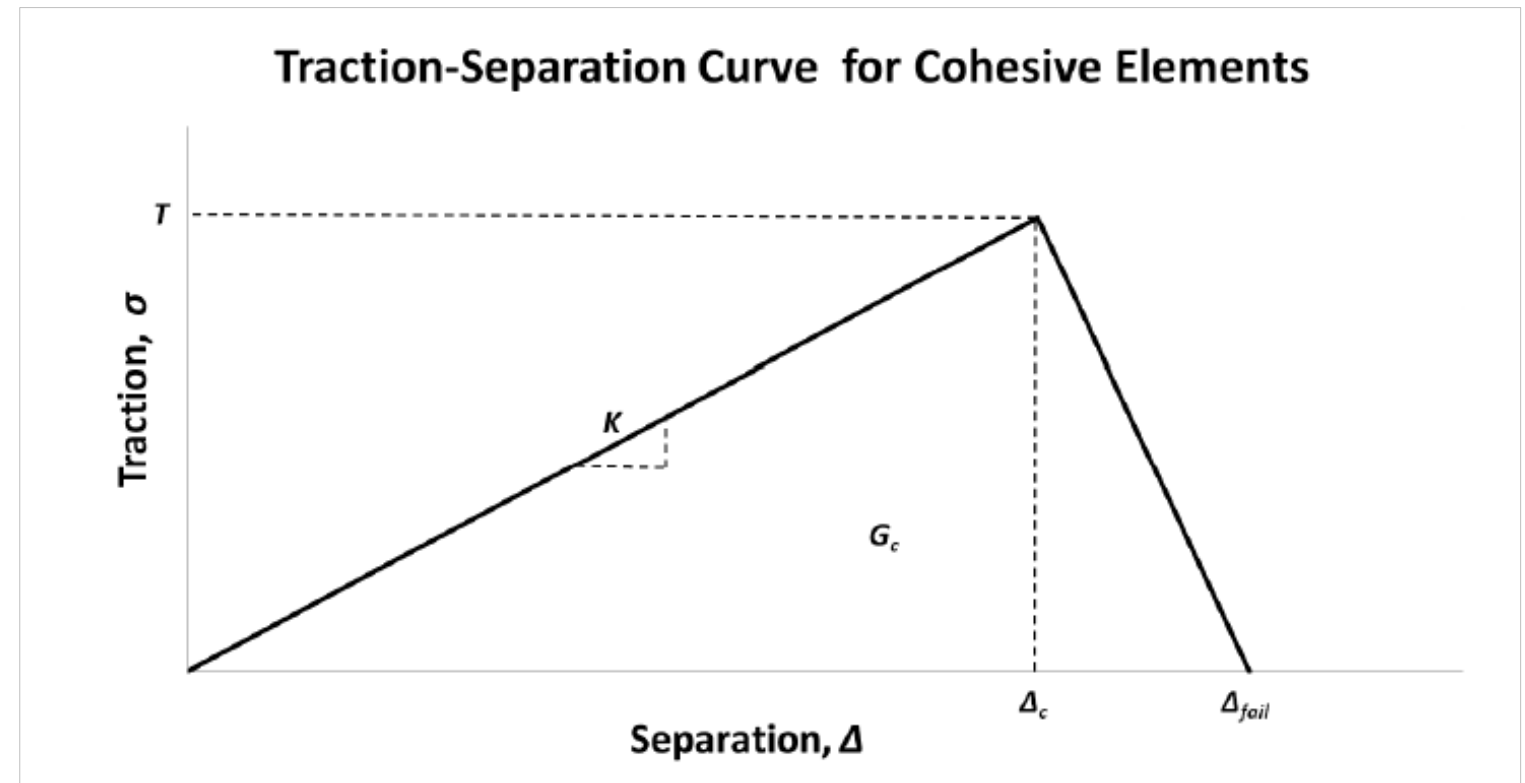

Figure 42: Traction-Separation curve for bi-linear criteria defining cohesive element damage.

\section{Damage Initiation}

*DAMAGE INITIATION values determine when the cohesive elements begin to damage and lose stiffness. While *DAMAGE INITIATION can be defined by many different criteria including various strains, stresses, and failure criteria (Abaqus Analysis User's Manual v6.12, 2012), the model developed here uses a simple stress-based damage initiation. In the bi-linear criterion, this determines the stress level at which the initial, positive sloped portion of the curve ends and the negative slope begins. This value is chosen so that damage in the model occurs at similar load and full-field strain as test coupons.

To do this, the DIC data was analyzed to determine when damage begins. When strains become discretized between adjacent fiber bundles then damage is considered to have occurred. Since fiber waviness causes shear (Mode II) dominated failures, shear 
strain may appear to be the best strain field to investigate to determine damage initiation. However, due to questionable shear DIC data as discussed above, shear strain data could make damage initiation appear to occur prematurely. For this reason, longitudinal strain is used to determine when damage initiation has occurred. Once damage initiation is apparent, the corresponding longitudinal far-field strain is obtained. After doing this for multiple coupons, it was found that damage occurs at a longitudinal far-field strain of $1.1 \%$ to $1.2 \%$ strain. The damage initiation inputs were determined, as described below, so that damage would occur at the same far-field strain. It should be noted that the DIC data is the strain field on the surface and not internal. While surface damage has a larger effect on the DIC data, internal damage also affects the surface strain field through strain redistribution. Therefore, this method is considered valid for determining when damage initiates.

A model was run with a large *DAMAGE INITIATION value so that damage initiation did not occur by the strain value at which damage should initiate. The maximum cohesive element shear and normal stress magnitudes were investigated at this far-field strain in Abaqus CAE. Shear stresses were much larger than normal stresses which was expected because damage induced by fiber waviness is a shear dependent damage as found by Riddle, 2013.

The maximum cohesive element shear stress magnitude at this displacement was set to the *DAMAGE INITIATION value for both normal and shear inputs. This value was $60 \mathrm{MPa}$. The same *DAMAGE INITIATION value is input for both normal and shear. 
It is important to note that there are assumptions in the model resulting in correlation between all experimental and analytical comparators not being possible. This is explained in more detail below after this through-thickness fiber alignment assumption is discussed in detail in regards to the *DAMAGE EVOLUTION parameters and after experimental-analytical comparisons have been made.

\section{$\underline{\text { Damage Evolution }}$}

The *DAMAGE EVOLUTION keyword effectively defines the energy that the cohesive elements must dissipate before being deleted. This is the area under the curve in the bi-linear criterion in Figure 42. This effectively controls how large a separation the cohesive elements can undergo before their load carrying capability decreases to zero and they are deleted. The inputs are the Mode I and Mode II critical energy release rates of the resin along with a slight fabric dependence. These values are determined from DCB and ENF testing for Mode I and II respectively. As mentioned above, these tests were previously performed with the initial development of this model and were based on ASTM D5528-13 and ASTM WK22949 standards. The values were determined to be

806 and $1524 \frac{\mathrm{J}}{\mathrm{m}^{2}}$ for Mode I and Mode II respectively (Nelson, 2013). The previous code input these values directly for the *DAMAGE EVOLUTION inputs. However, given the discrepancy between assumptions made by the model and what is actually being tested, more accurate results can be achieved by increasing these values. This discrepancy as well as how to increase these values are now discussed.

A 2D model allows for complex geometry in two dimensions while the third dimension is a simple extrusion. The coupon width and height plane is the complex 
geometry plane for this model. Therefore, complex geometry cannot be included in any other plane. This lack of geometric complexity in the thickness-length plane is a valid assumption, however, this is not the case for the thickness-width plane. This assumes that the fiber bundles align perfectly between plies which is not the accurate. Fiber misalignment between plies can be seen in the coupon cross-section in Figure 43 below and the CT images in Figure 24 above. Two schematic cross-sections are included to show the difference in through-thickness complexity between the aligned and misaligned plies. These schematic cross-sections can be seen in Figure 44 in which fiber bundles are represented by gray rectangles separated by black lines which are resin-rich areas. Each cross-section is only two fiber bundle widths wide in order to simplify the figure. The coupon cross-section from Figure 43 is included to orient the reader. The configuration on the left is what is modeled in the 2D model, not allowing for complex throughthickness geometry. This is called the flush configuration. The actual cross-section looks more similar to configuration on the right in which the fibers do not align between plies. This is called the step configuration. This schematic shows the maximum misalignment between plies. While a 3D model would allow for this complex throughthickness geometry to be accounted for discretely, 3D modeling is considered outside the scope of the work herein. Therefore, the following method is used to allow the 2D model to account for the complexity to increase accuracy. 


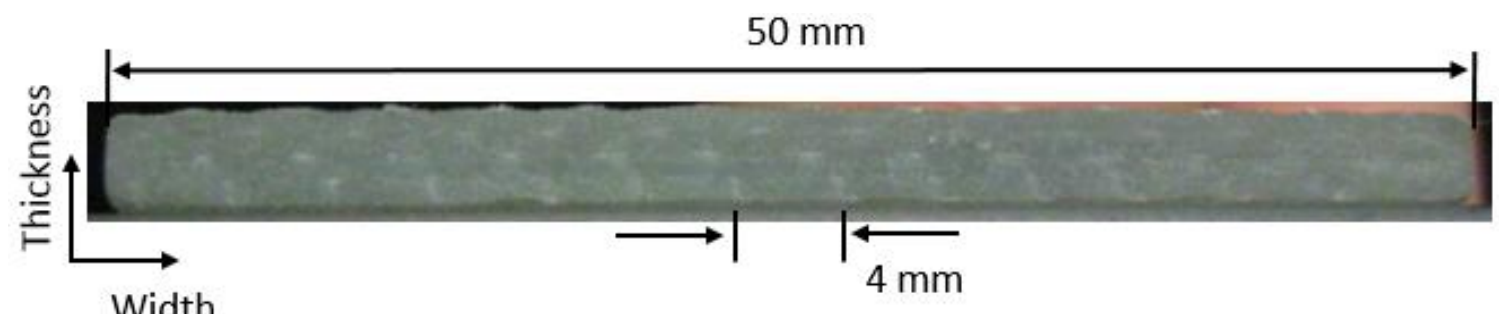

Figure 43: Picture looking down length of coupon with width and thickness directions labeled. Complex cross-section geometry of fiber bundles as well as resin rich location separation can be seen. $50 \mathrm{~mm}$ coupon width and $4 \mathrm{~mm}$ fiber bundle width labeled.

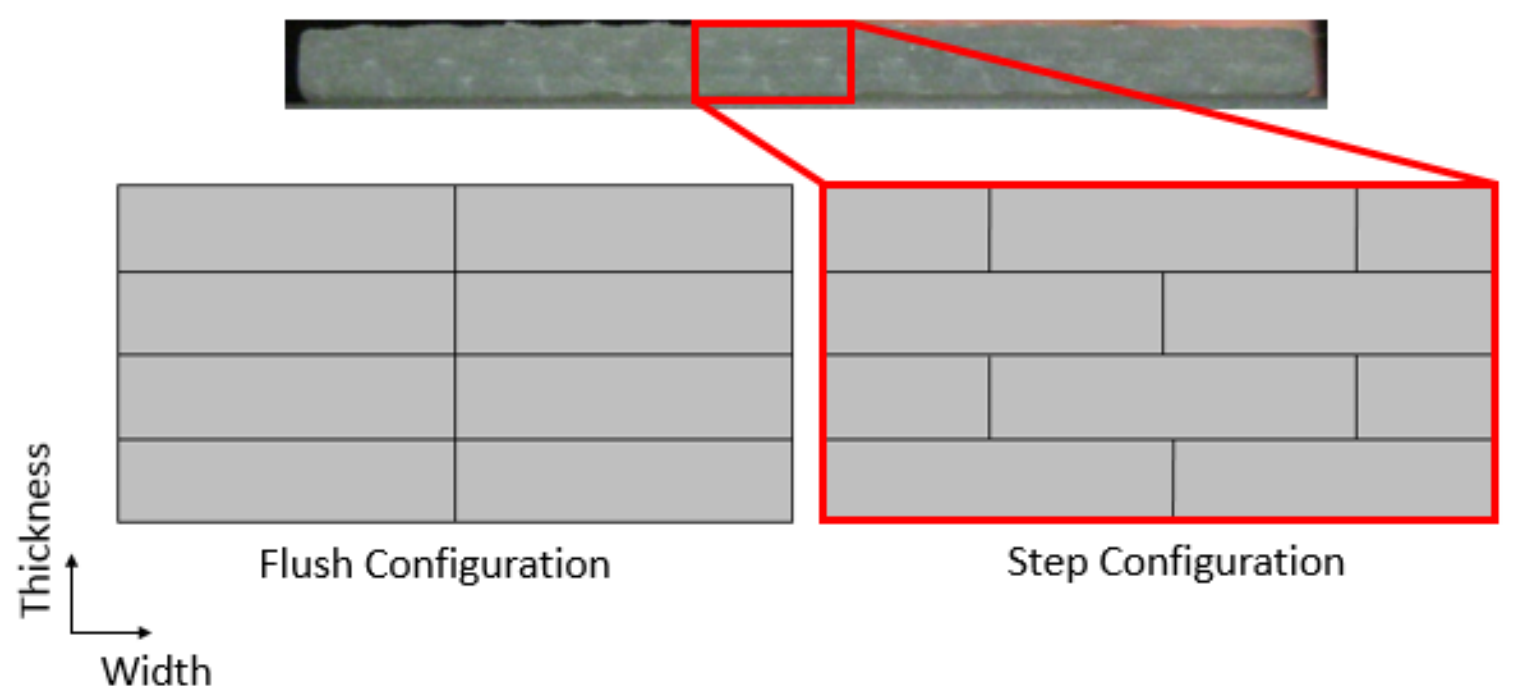

Figure 44: Schematic cross-sections of coupon geometry 2 fiber bundle widths wide and all 4 plies thick. Flush configuration with perfectly aligned bundles between plies as assumed by the model (left). Step configuration taking into account through-thickness complexity seen in actual coupon geometries (right).

The *DAMAGE EVOLUTION inputs are an energy per area. The total energy released before failure is the area multiplied by the critical energy release rate.

Therefore, if a crack must propagate farther, the energy released is larger. The distance that a crack needs to propagate for a through-thickness resin failure is shown for both configurations in Figure 45. It can be seen that the step configuration requires larger crack propagation than the flush configuration. This means that more energy must be released before bundles separate through the thickness for actual coupons with ply 
misalignment. The critical energy release rates from DCB and ENF testing can be used for *DAMAGE EVOLUTION inputs directly if the coupons being modeled have all fiber bundles in the flush configuration. Otherwise this assumption should be accounted for in the model.

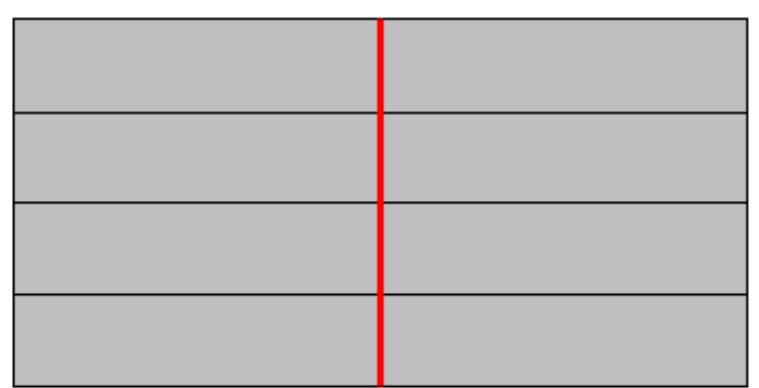

Flush Configuration

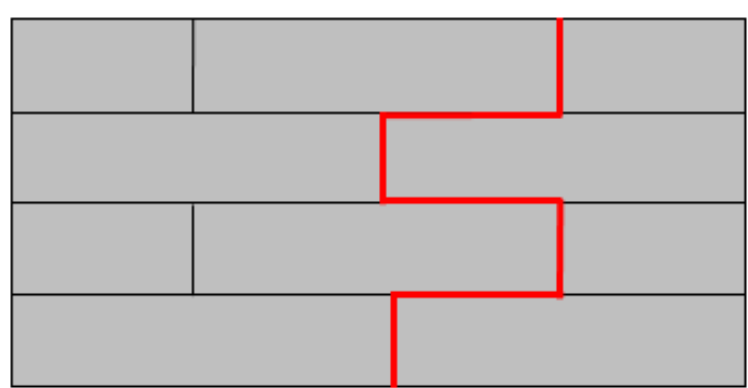

Step Configuration

Figure 45: Schematic cross-sections of 2 fiber bundles wide and all 4 plies thick. Flush configuration (left) and step configuration (right). Crack necessary for through-thickness resin failure shown by line.

There are two ways that this modeling-experimental geometry discrepancy can be accounted for. The first solution is to model the fiber bundles so that there is misalignment between fiber bundles in adjacent plies. This would require 3D modeling which is outside the scope of this work. The other solution is to change the ${ }^{*} D A M A G E$ EVOLUTION inputs to values such that when used in the flush configuration, they require the same total energy release for crack propagation as the step configuration with the experimental critical energy release rates. These updated values are called effective critical energy release rates. This will result in larger *DAMAGE EVOLUTION inputs. How much they can justifiably be increased is the next step. This can be done with hand calculations or with FE modeling. Both methods were used to compare against each other. The hand calculations are discussed first. 
The energy for a crack to propagate through-thickness in the flush configuration is less than that in the step configuration, given the same critical energy release rates, as shown in Equation 1. The goal of this process is to increase the critical energy release rates to effective values by setting the total energies for through-thickness crack propagation for both step and flush configurations, in both Mode I and II, to be the same as shown here:

$$
\begin{gathered}
E_{\text {crack flush }_{\text {flus }}} \leq E_{\text {crack }_{\text {step }}} \\
E_{\text {crack }_{\text {step }}}=E_{\text {crack }_{\text {flush }_{\text {eff }}}}
\end{gathered}
$$

Where $E_{\text {crack }_{\text {flush }}}$ and $E_{\text {crack }_{\text {step }}}$ are the energies required for a crack to initiate and propagate through the thickness in the flush and step configuration, respectively, with experimental critical energy release rates. $E_{\text {crack }_{f l u s h} \text { eff }}$ is the energy required for a crack to initiate and propagate through the thickness for the flush configuration which is set equal to that of the step configuration. The effective flush configuration uses effective critical energy release rates while the step configuration uses experimental critical energy release rates. These configurations, critical energy release rates, crack paths, and relevance are summarized in Table 7. 
Table 7: Summary of parameters used in the following discussion and calculations for flush, step, and effective flush configurations.

\begin{tabular}{|c|c|c|c|}
\hline $\begin{array}{c}\text { Mode I Critical } \\
\text { Energy Release Rate }\end{array}$ & $E_{\text {crack }_{\text {flush }}}$ & $E_{\text {crack }_{\text {step }}}$ & $E_{\text {crack }_{\text {flush }}}$ \\
\hline $\begin{array}{c}\text { Mode II Critical } \\
\text { Energy Release Rate }\end{array}$ & $G_{I c}$ & $G_{\text {Ic }}$ & $G_{\text {Iceff }}$ \\
\hline $\begin{array}{c}\text { Through Thickness } \\
\text { Crack Path }\end{array}$ & $G_{\text {IIc }}$ & $G_{\text {IIc }}$ & $G_{\text {IIceff }}$ \\
\hline Relevance & Originally Modeled & $\begin{array}{c}\text { Experimental } \\
\text { Coupons }\end{array}$ & \\
\hline
\end{tabular}

As mentioned above, the critical energy release rates from DCB and ENF testing are $G_{I C}$ and $G_{I I C}$ which are 806 and $1524 \frac{\mathrm{J}}{\mathrm{m}^{2}}$ respectively. The effective critical energy release rates to be calculated are $G_{\text {Iceff }}$ and $G_{\text {IIceff }}$. The through-thickness crack paths from Figure 45 are included again. The relevance is where these different configurations are used. The flush configuration with experimental values was originally modeled. The step configuration with experimental values is similar to the experimental coupons being tested and used for model accuracy comparisons. The effective flush configuration is what will now be modeled using the flush configuration, as before, but using effective critical energy release rates so as to have similar crack energies as experimental coupons.

The total energy for through-thickness crack propagation is calculated by multiplying the area and corresponding mode's critical energy release rate and summing with the other areas along the crack path. First, global Mode I loading is discussed. This 
means that the schematics above are loaded horizontally. This is shown for flush and step configurations with experimental values and flush configuration with effective values here:

$$
\begin{gathered}
E_{\text {Icrack }_{\text {flush }}}=A_{I} * G_{I c} \\
E_{\text {Icrack }_{\text {step }}}=A_{I} * G_{I c}+A_{I I} * G_{I I c} \\
E_{\text {Icrack }_{\text {flush }_{\text {eff }}}}=A_{I} * G_{\text {Iceff }}
\end{gathered}
$$

where $A_{I}$ is the crack propagation area which is Mode I dominated and $A_{I I}$ is the area which is Mode II dominated. $G_{I c}$ is the Mode I experimental critical energy release rate, $G_{I I C}$ is the Mode II experimental critical energy release rate, and $G_{I c e f f}$ is the Mode I effective critical energy release rate which will be determined. These equations rely on the assumption that the vertical cohesive elements are Mode I dominated and the horizontal cohesive elements are Mode II dominated when the model is loaded in global Mode I loading. Detailed calculations for energy of the flush configuration with experimental values $\left(E_{\text {Icrack }}\right.$ flush $)$ are not included because they are not necessary for determining the effective crack energy which is necessary for determining the effective critical energy release rates. However, this energy value is included below for comparison.

An important note here is that the experimental Mode I and Mode II critical energy release rates from DCB and ENF testing are assumed to be the same whether between fiber bundles in the same ply or neighboring plies. This should be the case if the composite was just unidirectional fiber bundles and resin. However, due to the stitching and backing fibers affecting interply and intraply crack energies differently, the critical 
energy release rates are likely slightly different between fiber tows within a fiber mat and between neighboring mats. Despite this variation between energy release rates, this is considered a valid method for magnitude adjustment.

The Mode I and Mode II dominated areas for Equation 2 are as follows:

$$
\begin{gathered}
A_{I}=n * t * d \\
A_{I I}=(n-1) * \text { ply overlap } * d
\end{gathered}
$$

where $\mathrm{n}$ is the number of plies, $\mathrm{t}$ is the ply thickness, $\mathrm{d}$ is depth, and ply overlap is the minimum distance between the edge of a fiber bundle in one ply to the nearest edge of a fiber bundle in the next ply. The actual area of the horizontal cohesive elements depends on the overlap between plies. This varies between coupons as well as within coupons. For this calculation, the effective values for the maximum overlap are calculated. The maximum overlap results in half the bundle width at each ply-ply interface. For a bundle width of $4 \mathrm{~mm}$, maximum ply overlap is $2 \mathrm{~mm}$. Plugging in for the step configuration in Mode I, the crack energy is:

$$
\begin{gathered}
E_{\text {Irack }_{\text {step }}=4 \mathrm{~mm} *} 806 \times 10^{-6} \frac{\mathrm{J}}{\mathrm{mm}^{2}} * d+6 \mathrm{~mm} * 1524 \times 10^{-6} \frac{\mathrm{J}}{\mathrm{mm}^{2}} * d \\
E_{\text {Irrack }_{\text {step }}}=12.368 \times 10^{-3} * d \\
E_{\text {Icrack }_{\text {step }}}=12.368 \times 10^{-3} \frac{\mathrm{J}}{\mathrm{mm}}
\end{gathered}
$$

This is the crack energy per unit depth. Depth is not important for these calculations so is omitted for now and energies per area are discussed. Plugging in for the flush configuration in Mode I, the crack energy is:

$$
E_{\text {Icrack }} \text { flush }_{\text {eff }}=4 \mathrm{~mm} * G_{\text {Iceff }}
$$


Equating the energies for each configuration and solving for $G_{I c e f f}$, results in:

$$
G_{\text {Iceff }}=\frac{12.368 \times 10^{-3} \frac{\mathrm{J}}{\mathrm{mm}}}{4 \mathrm{~mm}}=3092 \frac{\mathrm{J}}{\mathrm{m}^{2}}
$$

The maximum effective Mode I critical energy release rate is calculated to be $3092 \frac{\mathrm{J}}{\mathrm{m}^{2}}$, which is nearly 4 times larger than the $G_{\mathrm{I} c}$ value from testing of $806 \frac{\mathrm{J}}{\mathrm{m}^{2}}$.

A similar process can be carried out to solve for the maximum effective Mode II critical energy release rate. Now the energy calculated for the crack to fully propagate is for crack propagation due to global Mode II loading rather than Mode I. This means that one side of the configurations in Figure 45 are pushed into the page while the other is pulled out. With this loading, all areas of the crack surface are failing in Mode II, whereas before it was a mix of Mode I and II. Therefore, the energy for the crack to fully propagate is still defined as above but now the $A_{I}$ and $A_{I I}$ values are different. The crack energy equations for flush and step configurations with experimental values in Mode II loading are shown here as well as flush configuration with effective critical energy release rate in Mode II loading:

$$
\begin{gathered}
E_{\text {IIcrack }_{\text {flush }}}=A_{I_{\text {flush }}} * G_{\mathrm{II} c} \\
E_{\text {IIcrack }_{\text {step }}}=A_{I} * G_{\mathrm{I} c}+A_{I_{\text {step }}} * G_{\mathrm{II} c} \\
E_{\text {IIcrack }_{\text {flush }_{\text {eff }}}}=A_{\text {II }_{\text {flush }}} * G_{\text {IIceff }}
\end{gathered}
$$

The assumption now is that all cohesive element failures are Mode II dependent. This means that there is no $G_{\mathrm{I} c}$ dependence. The areas are now subscripted with "flush" and "step" because the entire crack surface is now in a single mode which varies between 
both configurations. The energy for the crack to fully propagate in the step configuration is calculated here:

$$
\begin{gathered}
E_{\text {IIcrack }_{\text {step }}=} 0 * d * 806 \times 10^{-6} \frac{\mathrm{J}}{\mathrm{mm}^{2}}+(4 \mathrm{~mm}+2 * 3 \mathrm{~mm}) * d \\
* 1524 \times 10^{-6} \frac{\mathrm{J}}{\mathrm{mm}^{2}} \\
E_{\text {IIcrack }_{\text {step }}}=15.240 \times 10^{-3} * d \\
E_{\text {IIcrack }_{\text {step }}}=15.240 \times 10^{-3} \frac{\mathrm{J}}{\mathrm{mm}}
\end{gathered}
$$

This is again the energy per unit depth for the step configuration. Setting this energy equal to the energy for the flush configuration results in:

$$
\begin{gathered}
E_{\text {IIcrack }_{\text {flush }}}=4 \mathrm{~mm} * G_{\text {IIceff }} \\
G_{\text {IIceff }}=\frac{15.240 \times 10^{-3} \frac{\mathrm{J}}{\mathrm{mm}}}{4 \mathrm{~mm}}=3810 \frac{\mathrm{J}}{\mathrm{m}^{2}}
\end{gathered}
$$

The maximum effective Mode II critical energy release rate is $3810 \frac{\mathrm{J}}{\mathrm{m}^{2}}$. Both maximum effective values have now been calculated. However, these calculations relied on the assumption that the cohesive element failures were Mode I or II dominated as mentioned above. This assumption is checked with a model as described below.

As mentioned above, these calculations relied on the assumption that Mode I and Mode II failures occur separately from one another. Investigation into this assumption through modeling in Abaqus is now discussed. Due to the models being run not containing the assumption of Mode and Mode II dominated failures, the validity of this assumption can be determined. These models are looking through the thickness, the same as in the hand calculations above. From these model runs, energy is calculated and 
compared to the crack energy from the hand calculations. If the crack energies are close then the assumption is accurate and the $G_{c e f f}$ values calculated above are assumed to be a good approximation.

The two models here look at the two interface configurations, flush and step. The meshes for these both look the same because the only difference are the cohesive elements which are zero thickness and follow the red paths. These are shown in Figure 46 and Figure 47. Figure 46 shows the models with red lines indicating cohesive elements and, therefore, the crack. Figure 47 shows the models after the cohesive elements have failed. While any thickness value can be used, a thickness of $0.5 \mathrm{~mm}$ is input in order to have elements of $1 \mathrm{~mm}^{3}$ volume, similar to the coupon model. Both models have horizontal cohesive elements spaced $1 \mathrm{~mm}$ apart to simulate the different plies. The difference lies in the vertical cohesive element placement which determines the crack propagation. 

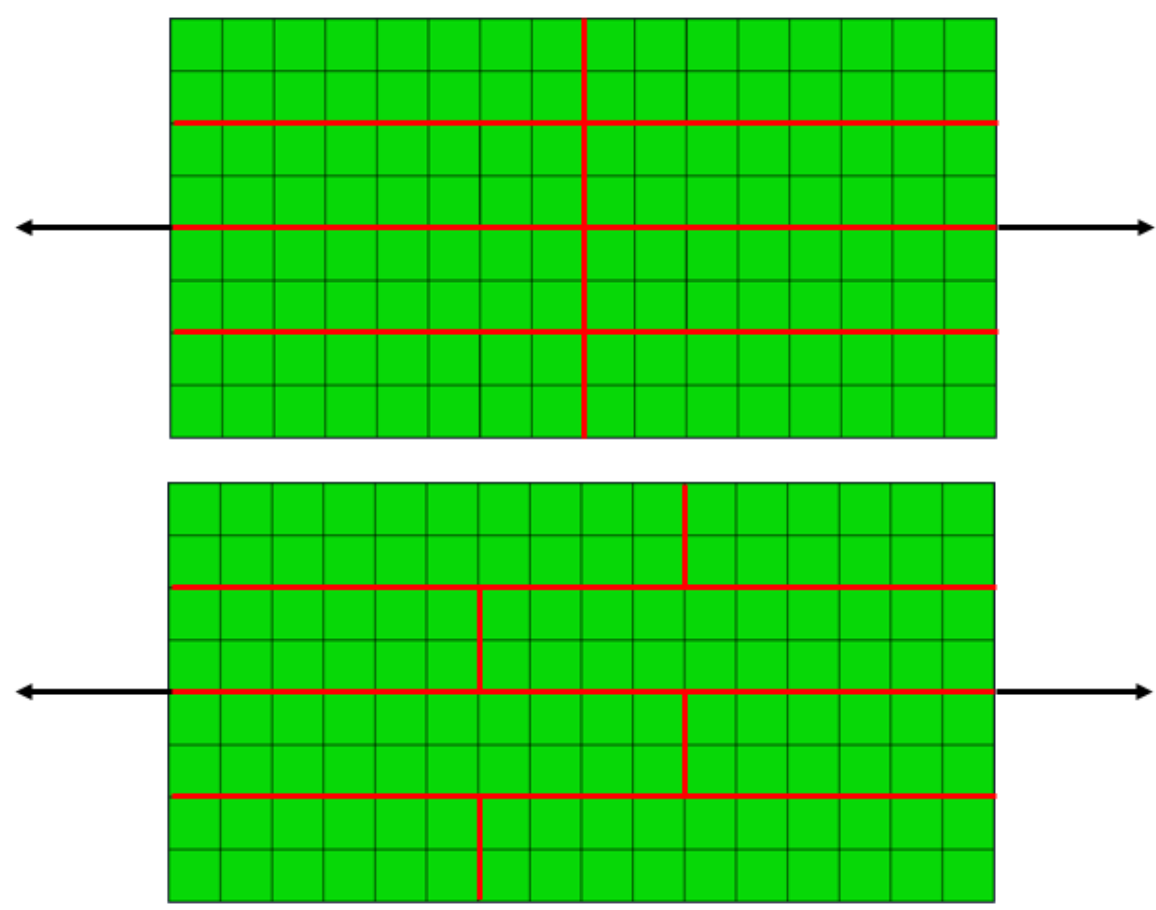

Figure 46: Models for calculating energy for crack propagation in Mode I for both flush configuration (top) and step configuration (bottom). Crack shown in red. Arrows depict global Mode I loading direction.
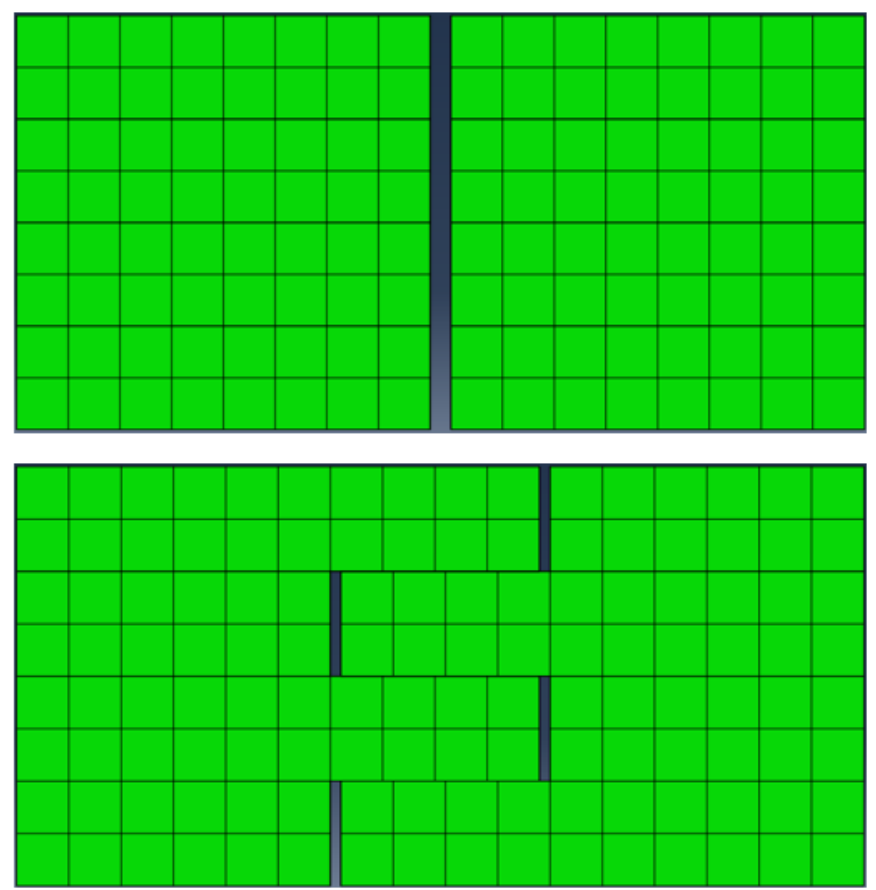

Figure 47: Models for calculating energy for crack propagation in Mode I for both flush configuration (top) and step configuration (bottom) after cohesive elements have failed. 
The right side nodes are constrained horizontally and the left side nodes are displaced horizontally. Runs were performed with the side nodes both constrained vertically as well as not constrained. Both had similar results but the unconstrained model values were used here because this is more accurate of the coupon test.

These models are used to calculate crack energy which is a check to make sure the energy used to calculate $G_{\text {Iceff }}$ is a good approximation with the assumptions for Mode I and Mode II dominated cohesive elements as mentioned above. Calculating the crack energy is achieved by entering the experimental $G_{\mathrm{I} c}$ and $G_{\mathrm{II} c}$ values into the step model. This model is loaded horizontally which simulates Mode I failure in the flush model. A Load-Displacement curve is created from this case and is shown in Figure 48. This shape shows that even for a simple bi-linear traction separation criteria, throughthickness complexity can cause large changes in both total energy as well as response shape. The energy released by the crack propagation is equal to the area under this curve which is calculated using the trapezoid rule. This yielded a crack energy of $6.2 \mathrm{~mJ}$. This is divided by thickness to get an energy per depth in order to compare to the hand calculations above. This results in a crack energy of $12.4 \frac{\mathrm{mJ}}{\mathrm{mm}}$. This is very close to the hand calculation of $12.368 \frac{\mathrm{mJ}}{\mathrm{mm}}$. Therefore, the assumption in the $G_{\mathrm{I} c e f f}$ calculations under the global Mode I loading that vertical cohesive elements are Mode I dominated and horizontal are Mode II dominated is considered to be valid. 


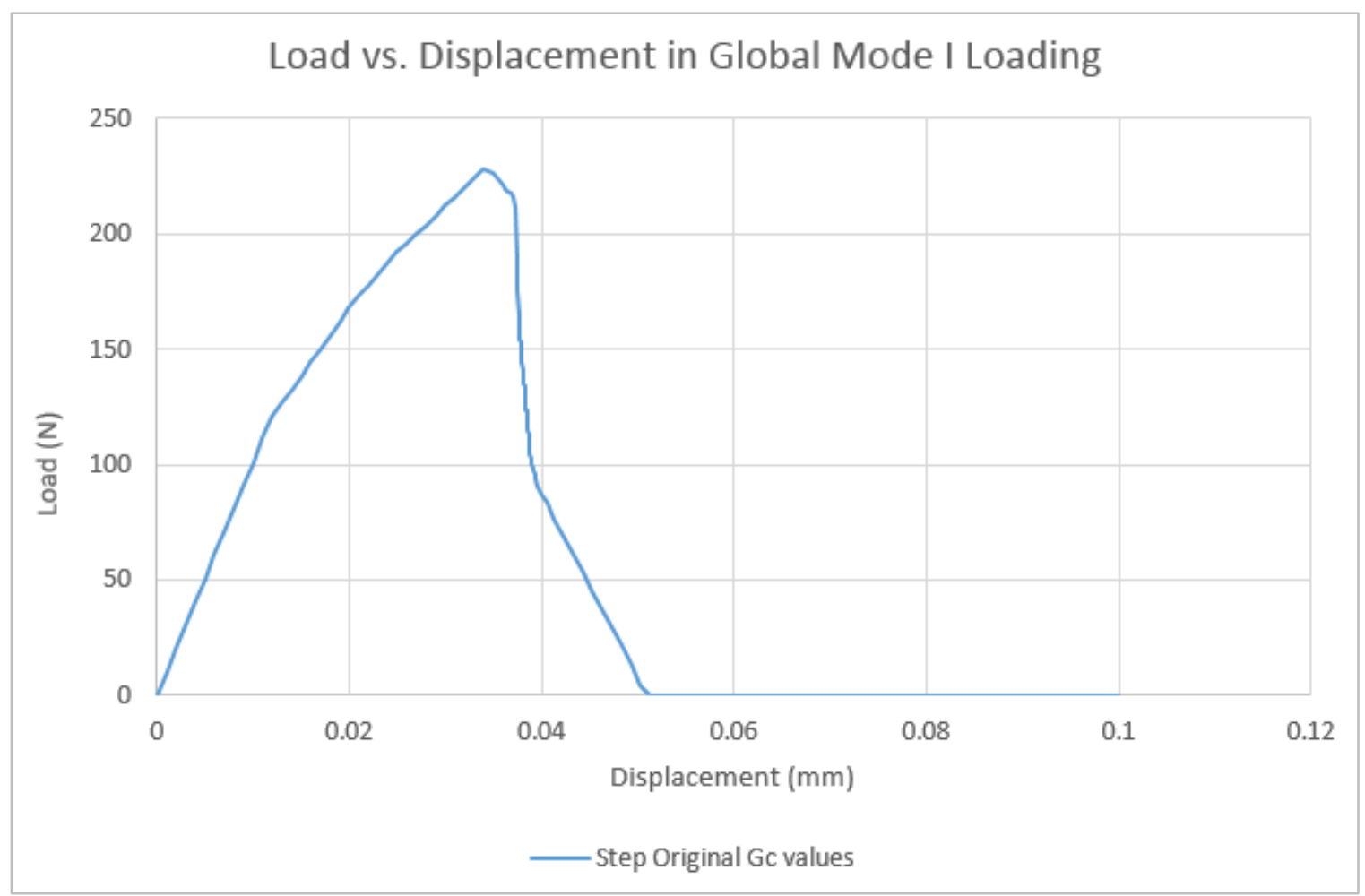

Figure 48: Load-Displacement curve for step configuration model in Mode I loading.

Now a check is performed to verify that the energy for the crack to propagate in the flush interface with the above calculated $G_{\text {Iceff }}$ value is in fact the same as the step model with original $G_{c}$ values. Therefore, $3092 \frac{\mathrm{J}}{\mathrm{m}^{2}}$ is entered into the flush model which is then displaced. $G_{\text {IIceff }}$ does not have to be calculated for this check because it has been seen that the flush model does not have $G_{\mathrm{II} c}$ dependence when loaded horizontally. A Load-Displacement curve was created and is shown, along with the step loaddisplacement curve, in Figure 49. The energy is calculated from this curve is the same as the step model and the energy is, again, $6.2 \mathrm{~mJ}$. This means that both models do in fact release the same amount of energy from crack propagation. 


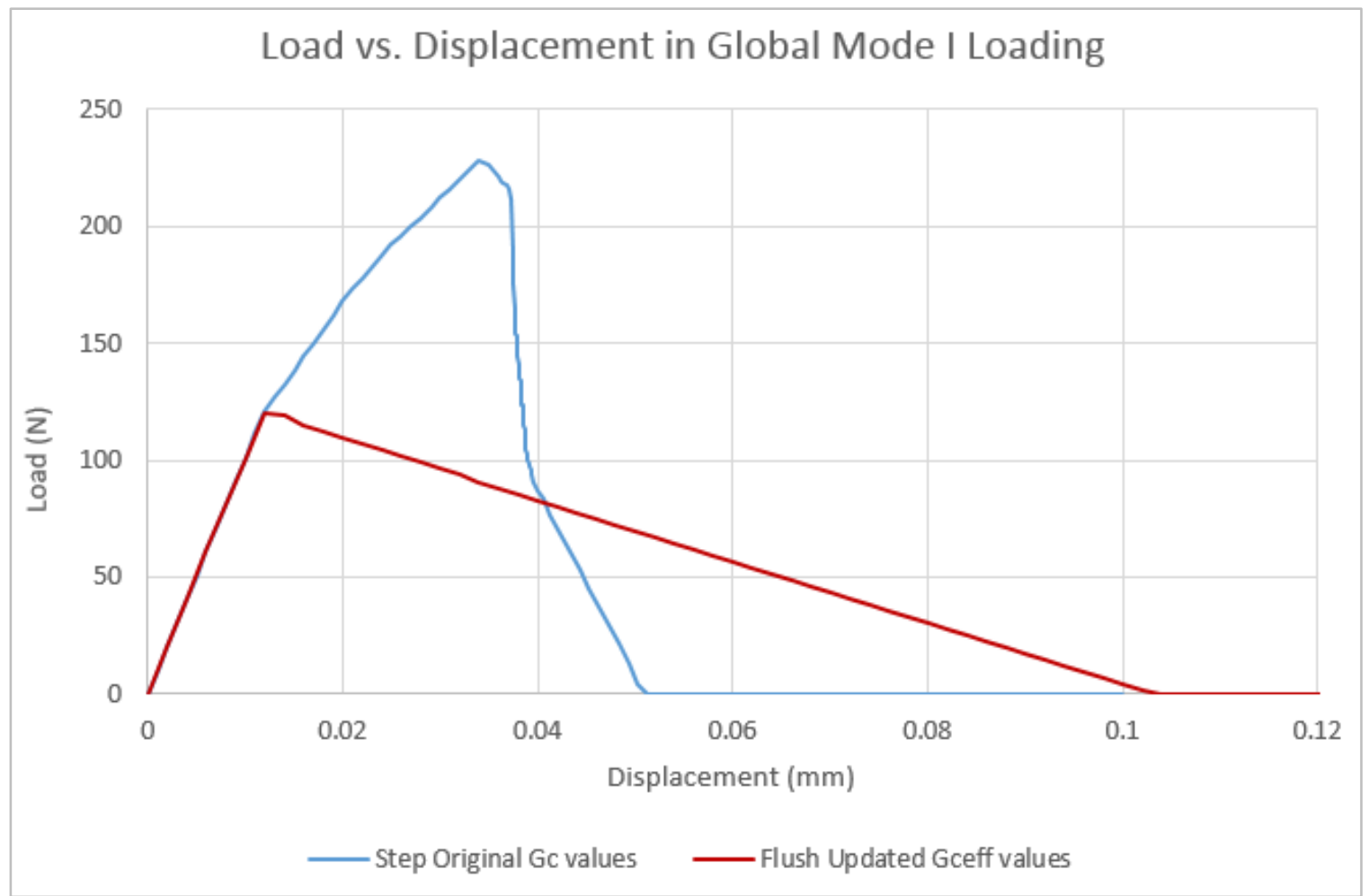

Figure 49: Load-Displacement curves for step and flush configuration models. Step configuration with original $G_{c}$ values and flush configuration with maximum effective $G_{c}$ values.

Although both models release the same amount of energy, they have different responses. This is because the horizontal cohesive elements in the crack path in the step model cause a more complex interaction than a bi-linear criterion can capture.

The flush configuration response from Figure 49 is be discussed first. The load increases until a load of $120 \mathrm{~N}$ is reached which causes the *DAMAGE INITIATION criteria to have been met by the vertical cohesive elements. This is expected for *DAMAGE INITIATION set at $60 \mathrm{MPa}$, due to the cross-sectional area of $2 \mathrm{~mm}^{2}$. Through multiple runs with various *DAMAGE INITIATION values it has been seen that the *DAMAGE INITIATION criteria affects curve shape but not the total crack energy. Therefore, *DAMAGE INITIATION values can change between this damage evolution 
investigation and the final coupon model without requiring *DAMAGE EVOLUTION values to be recalculated. Once damage initiation starts to occur, the cohesive elements soften as defined by the *DAMAGE EVOLUTION keyword in the bi-linear criterion. *DAMAGE EVOLUTION controls the softening because the second portion of the bilinear criterion is controlled by the energy required to be released before deletion of the cohesive element. The ${ }^{*} D A M A G E E V O L U T I O N$ input is this required crack propagation energy. Therefore, *DAMAGE EVOLUTION also controls the separation at which the cohesive element is deleted. The cohesive elements maintain load carrying capability until about $0.1 \mathrm{~mm}$ separation at which point they are deleted and the two halves of the model are no longer connected.

The step load-displacement curve is now discussed. Up until the load of $120 \mathrm{~N}$, before cohesive elements are damaged, this curve is the same as the flush curve. After damage has initiated, this curve has only a slight loss in stiffness and continues climbing to higher loads. This is because the vertical elements (Mode I dominated) damage at this load, similar to the flush model, however this configuration contains horizontal elements (Mode II dominated) which are not yet damaged. These horizontal elements allow for increasing load carrying capability not present in the flush model. The load stops climbing when the horizontal elements become damaged. The very steep drop in load starts when the first vertical cohesive elements fail and continues until they have all failed, leaving only horizontal cohesive elements. This also happens to be the point when the curves cross. Once there are only horizontal cohesive elements, the curve is decreases linearly until all the cohesive elements have been deleted at around $0.05 \mathrm{~mm}$. 
Although the actual response is not achieved using effective critical energy release rates in the flush configuration, this results in a more accurate model than inputting the experimental $G_{C}$ values directly as the *DAMAGE EVOLUTION input parameters. This is because, while actual cohesive responses between fiber bundles are not captured in the model, the total integrated energy for crack propagation is captured. The effect of capturing energy and not direct response is discussed below. It is explained how, although overall analytical-experimental correlation is increased using this method, correlation of certain comparators decreases.

The importance of changing the *DAMAGE EVOLUTION inputs is now demonstrated with load-displacement curves. Figure 50 contains load-displacement curves for the flush configuration and step configuration, both with experimental values. This flush curve is the response originally being modeled before the above process of increasing energy for crack propagation. The step configuration is similar to that of the tested coupons. 


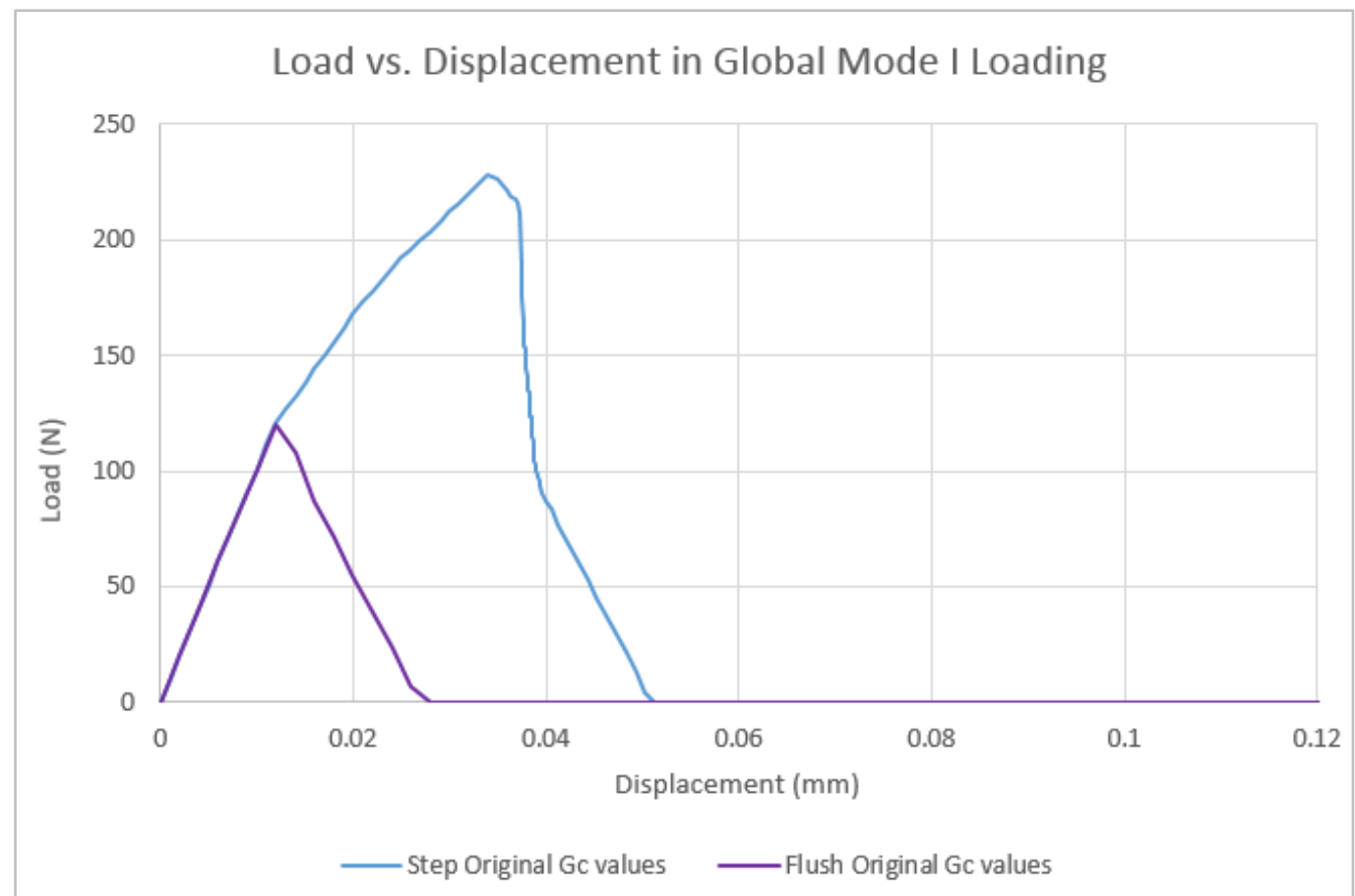

Figure 50: Load-Displacement curves for flush and step configurations, both with experimental $G_{C}$ values, demonstrating difference in energy to failure (area under curves).

Based on area under the curves, the flush configuration with experimental inputs requires a quarter of the energy for crack propagation than the step configuration with experimental inputs. This explains why the flush configuration with experimental values results in premature crack propagation compared to experiment.

The flush configuration with both experimental and updated $G_{C}$ values are now compared. Load-displacement curves for each can be seen in Figure 51. 


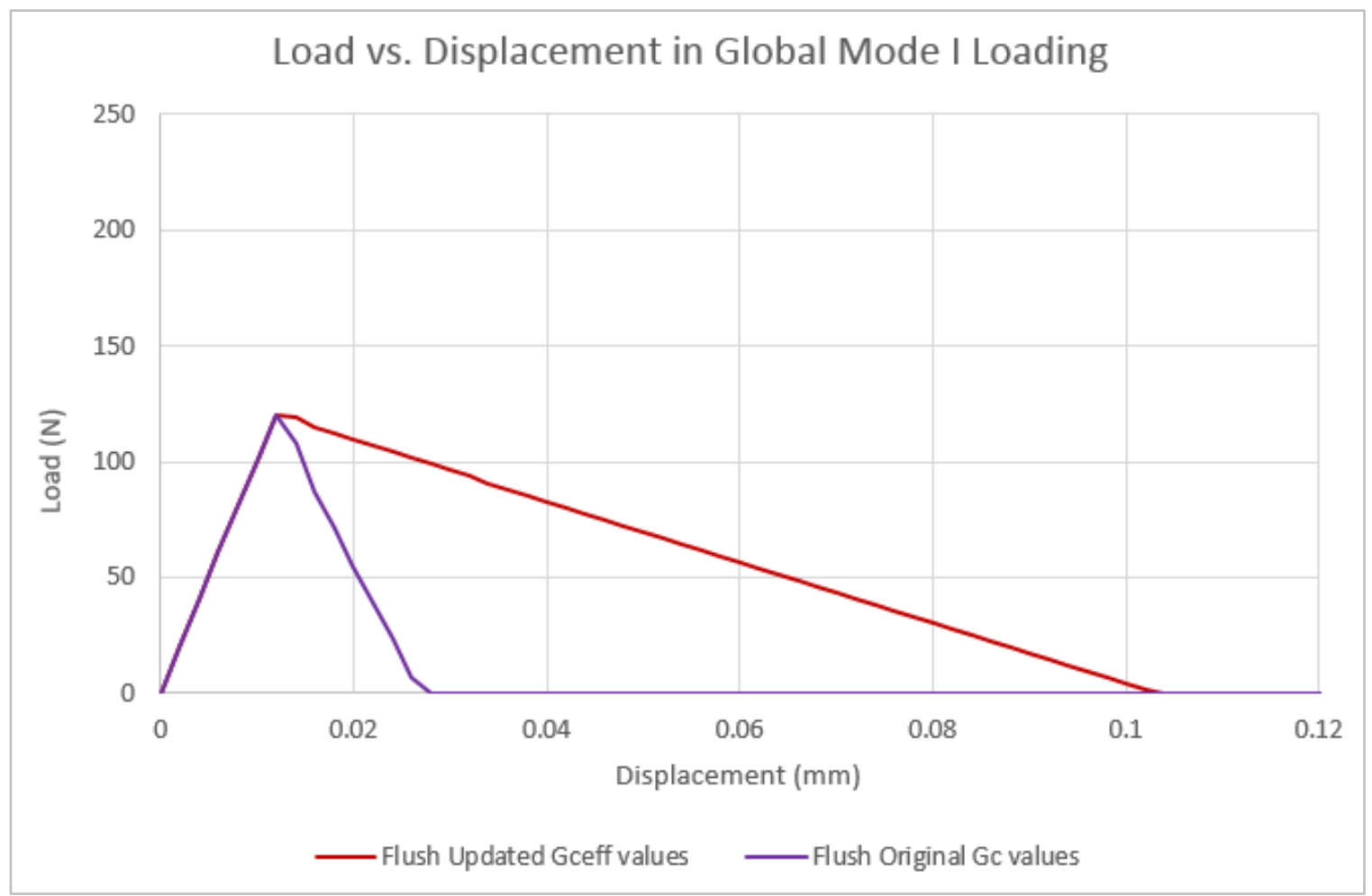

Figure 51: Load-Displacement curves for flush configuration with experimental values and updated values.

This plot includes the response of the cohesive elements both with updated $G_{c}$ values and with the original, experimental values. The response with experimental values is the response originally modeled and used in previous iterations of this modeling method. The curve for updated values is the response used for the model developed herein and discussed below. It can be seen that both models begin softening at the same displacement but the effective values result in a slower softening and larger crack energy than the experimental values.

Thus far, hand calculations have been performed to solve for $G_{\text {Iceff }}$ and $G_{\text {IIceff }}$ and, due to assumptions necessary in the calculations, model runs in Mode I global loading have been performed to check the total crack energy from which to calculate 
$G_{\text {Iceff }}$. Ideally, a similar modeling method would now be implemented to calculate the crack energy in Mode II global loading to then calculate $G_{\text {IIceff }}$. However, given the plane of geometry being modeled and the necessary direction of loading being normal to this, a 3D model would be required. The models from above would need one side to be pushed into the page and the other to be pulled out which is not possible with $2 \mathrm{D}$ elements. 3D modeling is outside the scope of this work. Given the accuracy of the hand calculations for $G_{\mathrm{II} c e f f}$ in Mode I global loading based on model comparison, these calculations are considered valid for Mode II loading for $G_{\mathrm{II} c e f f}$ calculations.

\section{$\underline{\text { Summary of Damage Evolution Correction }}$}

A summary and implications of this method of accounting for ply misalignment are now discussed. From this process we have determined maximum $G_{\text {Iceff }}$ and $G_{\text {IIceff }}$ values which are a combination of the $G_{C}$ material properties and the cross-sectional geometry. These values as *DAMAGE EVOLUTION inputs allows the complex throughthickness geometry to be more accurately represented in the model without actually changing the geometry of the coupon model. The $G_{\text {Iceff }}$ and $G_{\text {IIceff }}$ values were

determined to be 3092 and $3810 \frac{\mathrm{J}}{\mathrm{m}^{2}}$ respectively. These values are summarized in Table 8, similar to Table 7 above. Crack energy per unit depth is now also included. 
Table 8: Summary of experimental and calculated values for flush, step, and effective flush configurations from above process to determine effective critical energy release rates.

\begin{tabular}{|c|c|c|c|}
\hline & $E_{\text {crack flush }}$ & $E_{\text {crack }}$ step & $E_{\text {crack } \text { lush }_{\text {eff }}}$ \\
\hline $\begin{array}{c}\text { Mode I Critical } \\
\text { Energy Release Rate } \\
\qquad\left(\frac{\mathrm{J}}{\mathrm{m}^{2}}\right)\end{array}$ & 806 & 806 & 3092 \\
\hline $\begin{array}{c}\text { Mode II Critical } \\
\text { Energy Release Rate } \\
\qquad\left(\frac{\mathrm{J}}{\mathrm{m}^{2}}\right)\end{array}$ & 1524 & 1524 & 3810 \\
\hline $\begin{array}{l}\text { Global Mode I Crack } \\
\text { Energy per Depth } \\
\qquad\left(\frac{J}{m}\right)\end{array}$ & 3.2 & 12.4 & 12.4 \\
\hline $\begin{array}{l}\text { Global Mode II Crack } \\
\text { Energy per Depth } \\
\left(\frac{J}{m}\right)\end{array}$ & 6.1 & 15.2 & 15.2 \\
\hline $\begin{array}{l}\text { Through Thickness } \\
\text { Crack Path }\end{array}$ & & & \\
\hline Relevance & $\begin{array}{l}\text { Originally } \\
\text { Modeled }\end{array}$ & $\begin{array}{l}\text { Experimental } \\
\text { Coupons }\end{array}$ & Updated Model \\
\hline
\end{tabular}

This table includes the critical energy release rates used in each configuration for crack energy calculations. The crack energies per depth in Mode I and Mode II global loadings are also included. This table summarizes the impetus and results from this critical energy release rate adjustment. The crack energies for the flush configuration with experimental values are much lower than those for the step configuration. The critical energy release rates were updated to the effective critical energy release rates. 
The effective critical energy release rates used for the effective flush configuration are larger than experimental values. This results in the crack energies being equal for both the step and effective flush configurations. The Mode I effective critical energy release rate is 3.8 times larger than experimental and the Mode II effective critical energy release rate is 2.5 times larger.

However, as mentioned before, these are the maximum effective values for this fabric architecture if every ply-ply interface has the maximum overlap between each bundle. We have just determined the upper bound for *DAMAGE EVOLUTION input parameters that can be justifiable with this method. The lower bound was already known and is the experimental $G_{c}$ values. While the overlap of each bundle-bundle interface between plies in each coupon could be measured individually by looking at the ends, this may not be the same overlap through the length. Another option is a detailed statistical investigation of overlap between plies. This could include CT scans as above to investigate the fiber bundle misalignment within the coupon. However, these options are considered outside the scope of the work included here. Both of these options are deemed outside the scope of this work. Due to best model correlation being found using these maximum $G_{c e f f}$ values, these values are used for the final model discussed below.

This method accounts for complex through-thickness geometry with energy value only. This does not include the corresponding complex response. Inputting the actual response of the cohesive elements, as shown in the step response in Figure 48, is recommended to further improve the accuracy of this model. Rather than using bi-linear criteria for cohesive element response, more complex traction-separation responses can 
be input using the tabular modifier for the *DAMAGE EVOLUTION keyword. This could allow for the exact traction-separation response to be directly entered into the model. This could allow for both the crack energy and peak cohesive traction to both be captured in the model, whereas the bi-linear criteria only allows one to be represented.

The exact response for Mode II loading requires a simple 3D model similar to the 2D model used here for Mode I loading. As mentioned above, 3D modeling is outside the scope of this work. However, this seems to be a promising method to reduce the inaccuracy caused by modeling a complex $3 \mathrm{D}$ coupon with a $2 \mathrm{D}$ model. The exact response can be compared to the value obtained here with hand calculations. Because the traction-separation curve is likely different, the exact response can then also be directly input with the tabular modifier, same as recommended for $G_{\text {Iceff }}$.

Due to the update to the model herein including a change in cohesive element spacing, it should be noted that effective critical energy release rates calculated as performed above depends on the cohesive element spacing. The effective critical energy release rates are calculated using maximum bundle overlap. Maximum overlap is half the cohesive element spacing. Larger spacing means that more overlap is possible between plies which results in larger effective critical energy release rates.

The method outlined here for determining *DAMAGE EVOLUTION values is based on crack propagation energy. This method is required because the assumption of the $2 \mathrm{D}$ model that fiber bundles align through the thickness is not valid. The overlap causes an increase in crack length and, thus, an increase in energy for through-thickness crack propagation. While this method does not allow for the exact response between 
adjacent fiber bundles to be captured, this method does account for the increase in energy and is, thus, a step forward in capturing the actual response.

\section{Model with Updated Properties}

The Abaqus input file for model as described so far and used herein is included in the Appendix. Now that the cohesive material properties have been updated, failure criteria must be determined. Previous work with this modeling method used full-field longitudinal strain to determine when final failure had occurred. However, because the experimental work herein focuses on longitudinal strain over the wave, this will be the basis for failure criteria. Strain over the wave is calculated as the displacement across the wave divided by the length of the wave. This strain is used in the following discussion.

As mentioned above, average longitudinal strain over the wave at failure was $2.34 \%$ for the CFIP coupon. This strain was over the $50 \mathrm{~mm}$ wave which means that this resulted in a $1.17 \mathrm{~mm}$ displacement longitudinally over the wave. Although ultimate stress occurred at this strain, most coupons continued to carry load while softening, therefore, the model is not necessarily truncated at this strain. This is discussed below with the stress-strain curve.

Strain and stress in the model are calculated as follows. The strain is calculated by taking the displacement over the wave divided by the wave length of $50 \mathrm{~mm}$. The stress was calculated by taking the reaction force at the top node set and dividing by the cross-sectional area.

This model can be run to any displacement desired. This model was run up to a $2.56 \%$ wave strain to exceed the average failure strain of $2.34 \%$ and be on the high side 
of the experimental variability seen. It was expected that a strain level based on testing would be chosen to determine failure. However, the stress-strain curve can be seen in Figure 52 matches those from testing which makes a qualitative failure determination work well here. It can be seen that the stress peaks, drops, and rises again. Based on the experimental failures of these coupons, stress peaks, drops, and fails soon after. Very slight stress increases were seen in a few coupons. Therefore, this coupon should fail between the first stress peak and the following low stress before a large stiffness increase. These points are labeled with arrows as ultimate stress and final failure and are at $2.37 \%$ and $2.45 \%$ strain, respectively. These strains at ultimate stress and final failure are very close to those expected based on testing so both quantitative and qualitative determination of failure agree. This stress-strain curve truncated for failure is shown in Figure 53. The dashed line after the ultimate stress is to show that the coupon would be expected to reach final fail anywhere along that portion of the curve. 
111

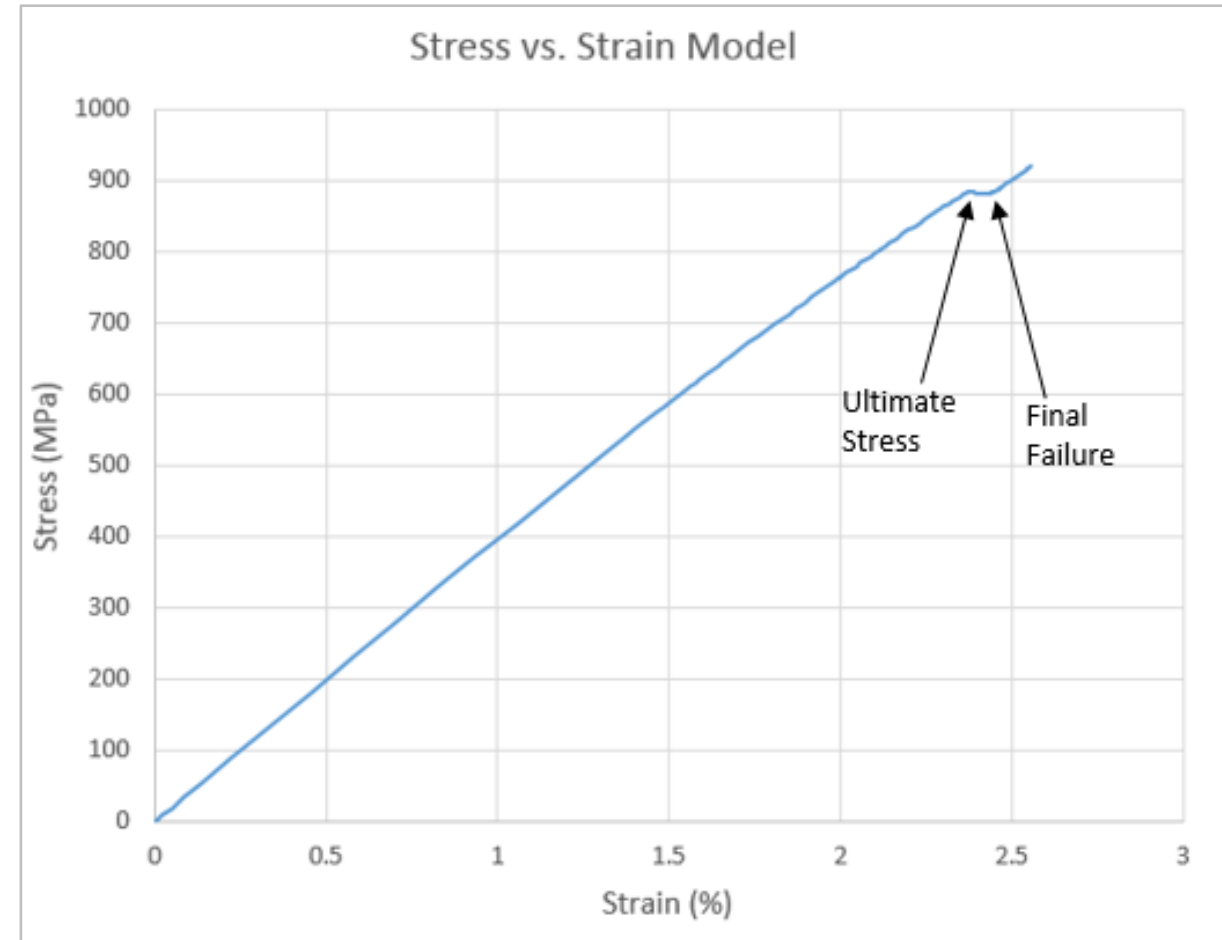

Figure 52: Stress vs. Strain plot for CFIP wave coupon model up to $2.56 \%$ strain. Failure and associated truncation has not been applied.

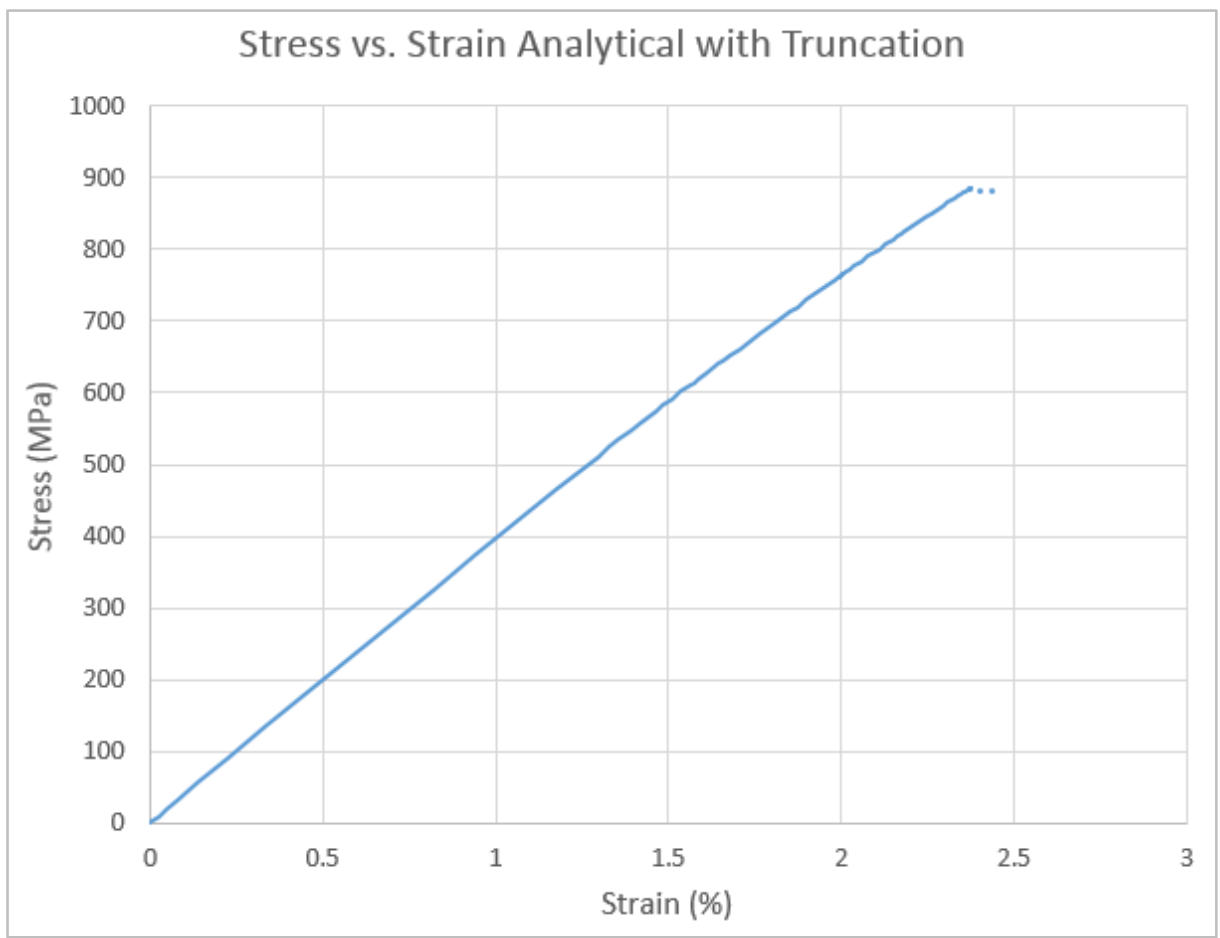

Figure 53: Stress vs. Strain plot for CFIP wave coupon model after failure criteria has been applied. Failure would occur somewhere on the dashed line. 
Now that failure has been determined, the analytical stress-strain response is discussed. The ultimate stress is $885 \mathrm{MPa}$ and the strain at failure is $2.37 \%$. There is a possibility of load carrying capability beyond ultimate stress, up to $2.45 \%$ strain. The initial elastic modulus is $39.8 \mathrm{GPa}$.

Strain fields of the modeled coupon are now discussed. The strain fields included are longitudinal, transverse, and shear in Figure 54, Figure 56, and Figure 57 respectively. These are taken from noted times during damage progression. The first image for each strain field is when the first cohesive element is damaged at $1.17 \%$ strain. The second image is when ultimate stress is reached at $2.37 \%$ strain. The third image is at $2.45 \%$ strain which is when the model is truncated and final failure will have occurred. The unit for strain in each image is $\mathrm{mm} / \mathrm{mm}$. 


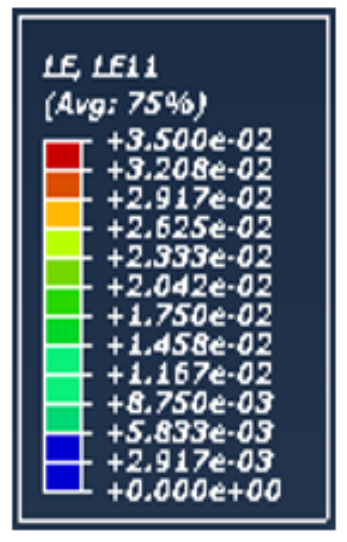

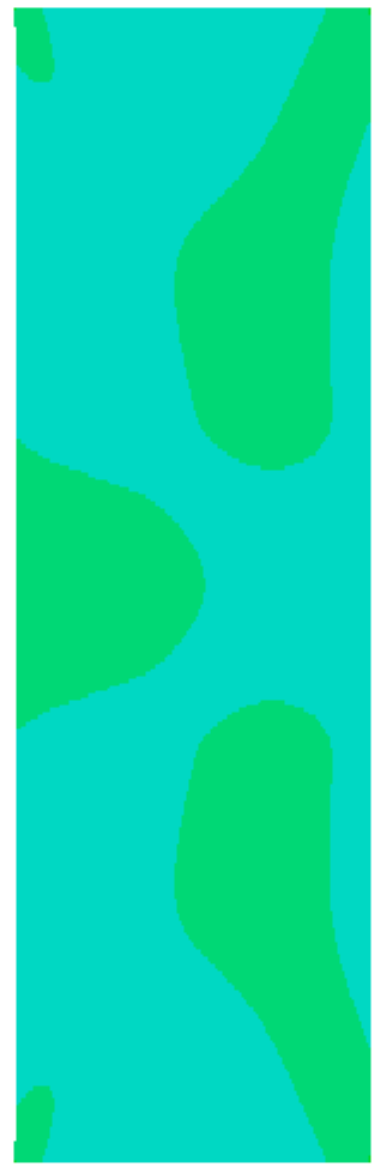

a

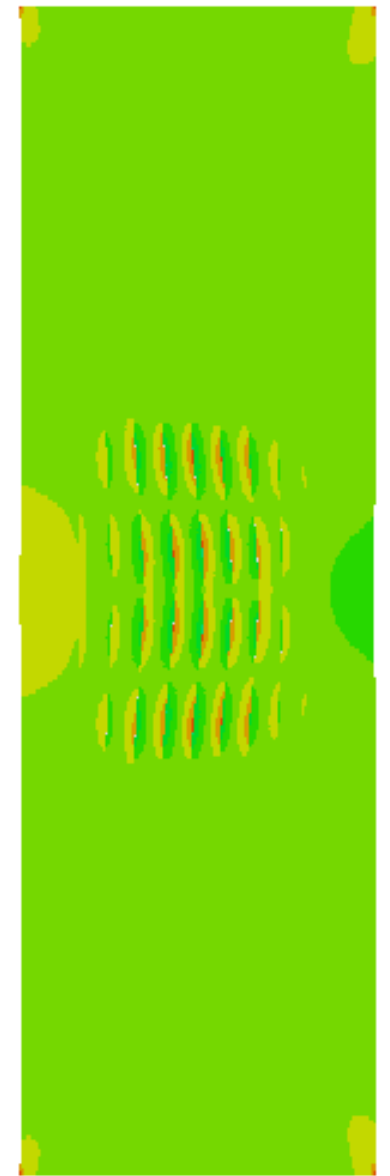

b

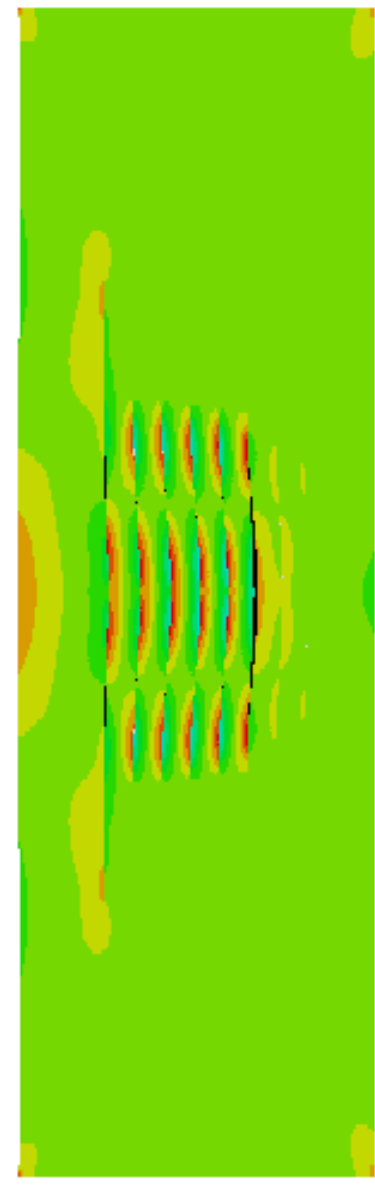

c

Figure 54: Longitudinal strain field at 1.17 (a), 2.37 (b), and 2.45 (c) \% longitudinal strain over the wave, showing damage progression. Strain values correspond to first cohesive element damage (a), ultimate stress (b), and final failure (c).

It can be seen that as the longitudinal strain accumulates early, high strain locations stem from each corner due to the boundary conditions, as well as in the left side in the wave region. This left side high strain location can be seen throughout loading. As cohesive elements damage and fail, this high strain region increases in magnitude and moves further to the left. Simultaneously, four bands of high strain across the width, two on either side of the wave, develop. These two high strain regions correspond to the two 
failure initiation locations discussed above, either left side tows or maximum misalignment.

The high strain bands occur around the four locations of maximum curvature. The magnitude is proportional to the degree of misalignment which is why the magnitude is larger near the center of the wave across the width. These bands are discussed in more detail on the single fiber bundle level.

A single fiber bundle near the middle of the coupon through the wave section at ultimate stress can be seen in Figure 55. This is a single fiber bundle from Figure 54 (b) above, which is included to show the fiber bundle location. The strain locations are called either the outer high strain region because they are farther outside the wave, or inner high strain region because they are more inside the wave. It can be seen that the outer high strain region appears on the right side of the fiber tow while the inner high strain region appears on the left side. On the opposite side of each high strain region in the fiber bundle, there is a low strain region. These high and low strain regions can be understood by thinking of the top and bottom of the fiber bundle in Figure 55 being fixed horizontally while loaded vertically. There is a shift left across the midline as the fiber bundle straightens. The outer high strain regions form because this shift and straightening causes more tension in the right side of the bundle than the left. Similarly, the inner high strain regions form because the shift and straightening causes more tension in the left side of the bundle than the right. 

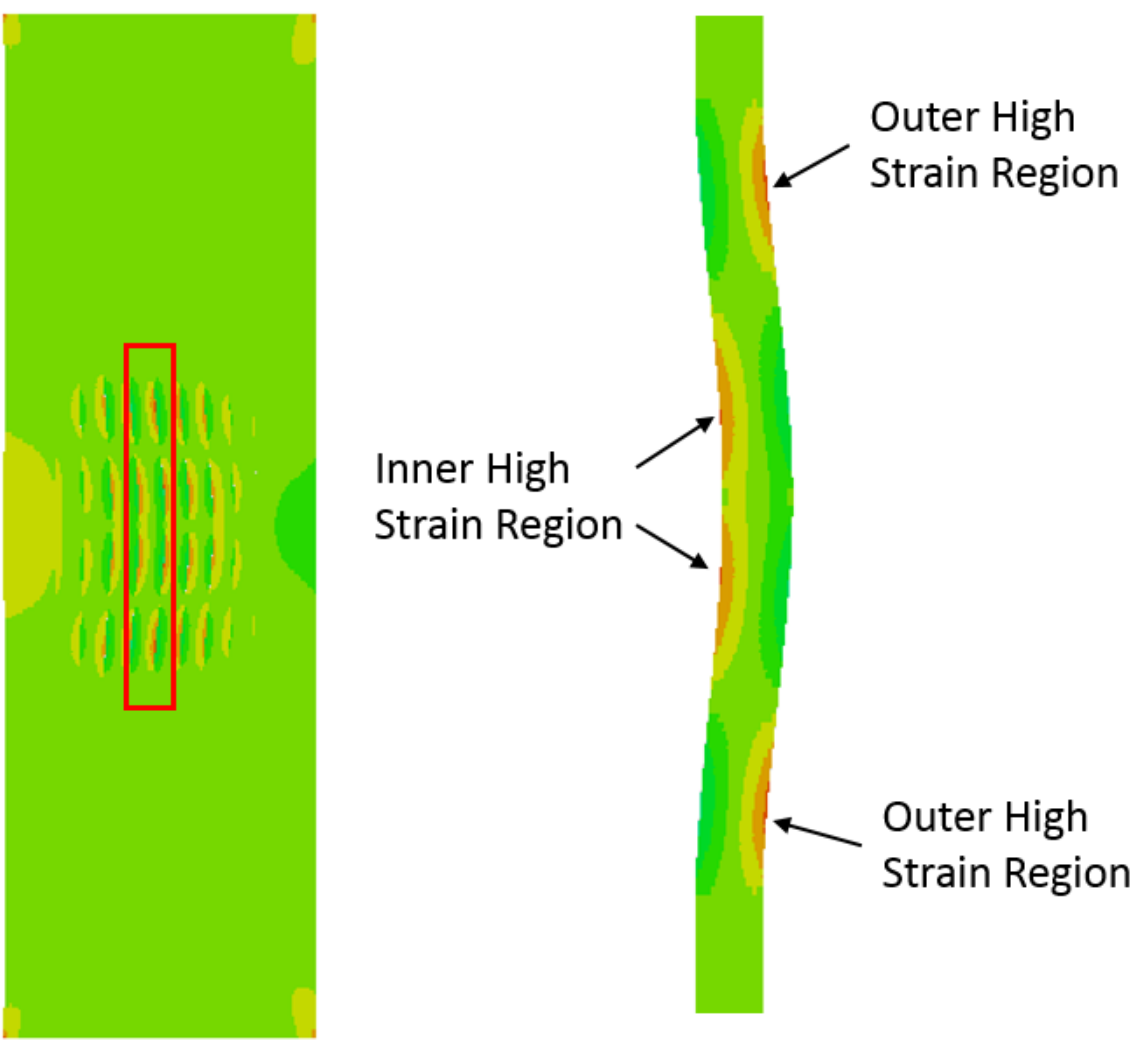

Figure 55: Single fiber bundle longitudinal strain distribution showing four regions of high strain near locations of maximum curvature. Coupon included showing location of fiber bundle investigated. 


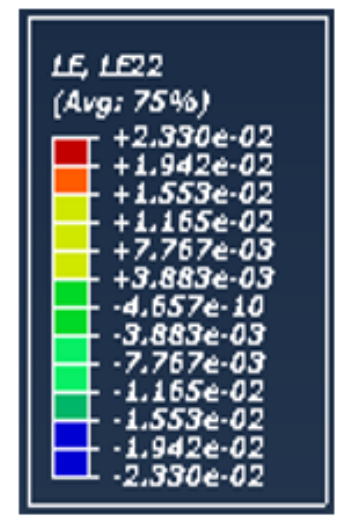

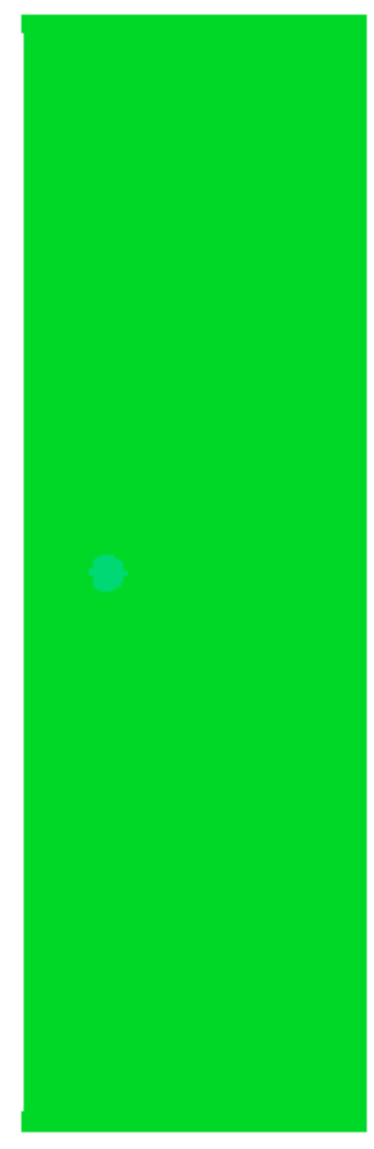

a

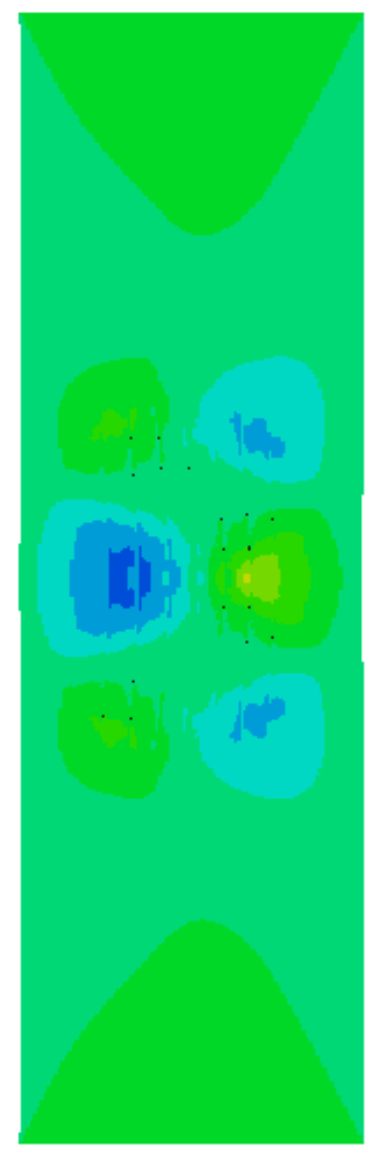

$\mathrm{b}$

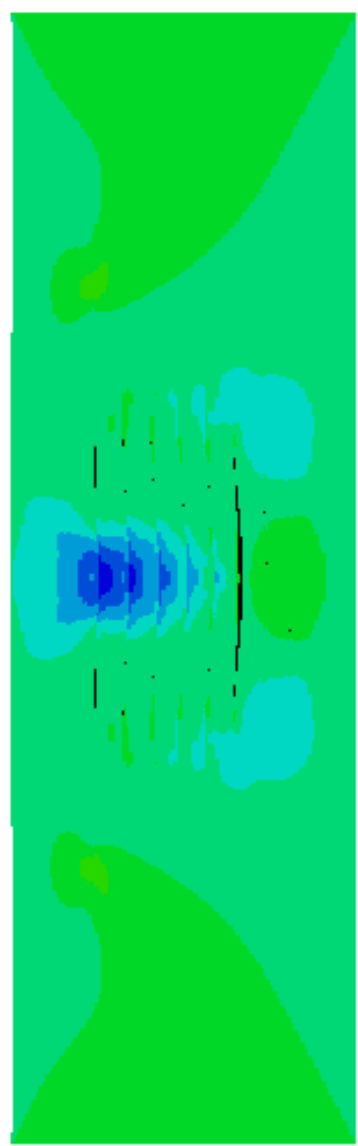

$\mathrm{c}$

Figure 56: Transverse strain field at 1.17 (a), 2.37 (b), and 2.45 (c) \% longitudinal strain over the wave, showing damage progression. Strain values correspond to first cohesive element damage (a), ultimate stress (b), and final failure (c).

The transverse strain starts off highest near the top and bottom of the coupon due to boundary conditions constraining the nodes horizontally. Within the wave, there is a region of strain accumulation on the left side. As loading continues, this strain location remains and increases. While this strain is negative, there is a positive strain location on the right side within the wave. Strain concentrations are also above and below the wave with negative strain on the right and positive on the left. As the cohesive elements fail, the negative strain area remains but the high positive strain region within the wave is relieved. 


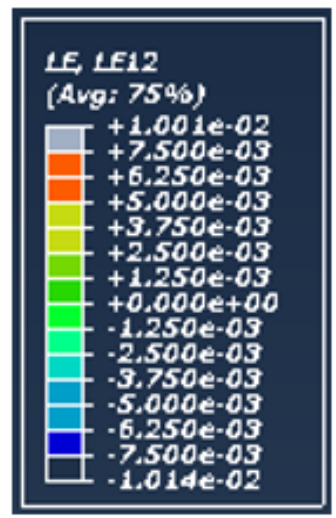

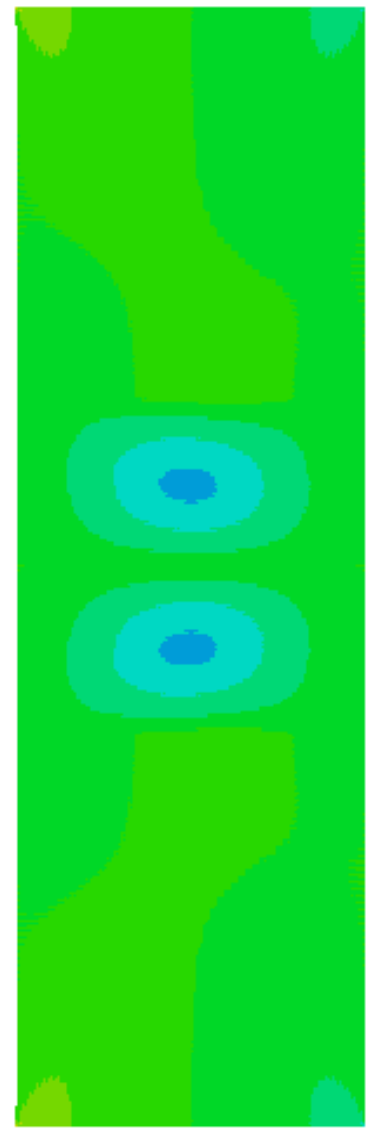

a

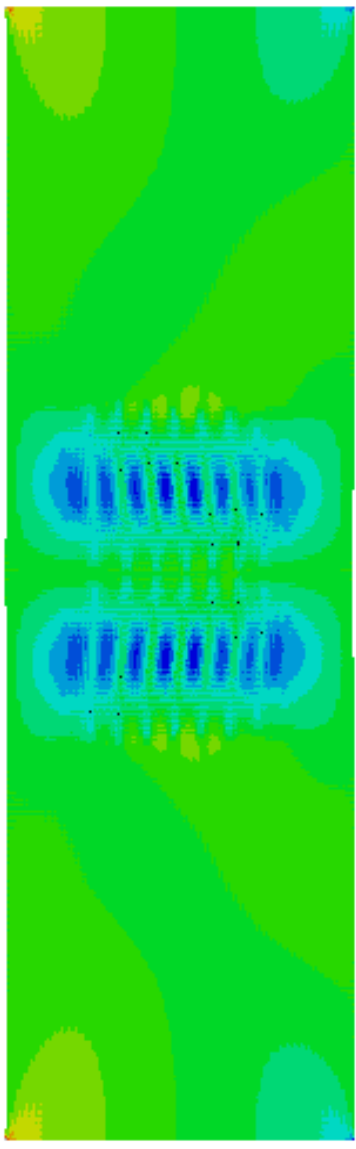

$\mathrm{b}$

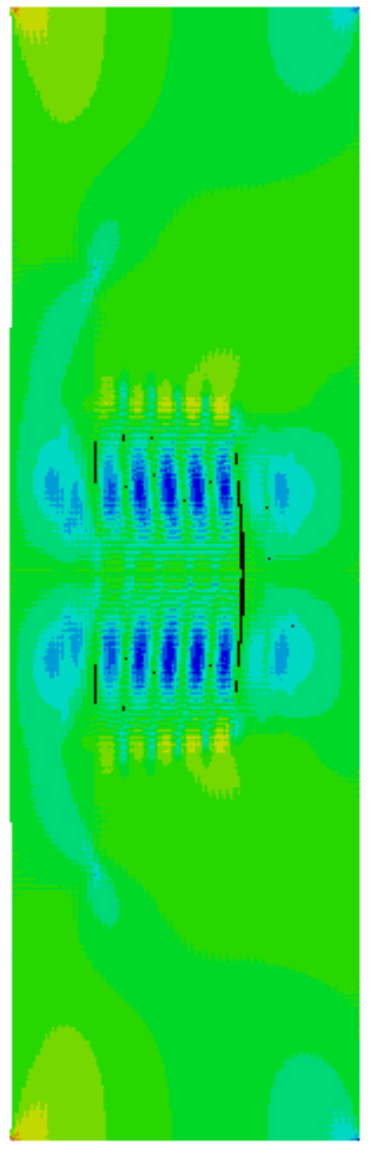

$\mathrm{c}$

Figure 57: Shear strain field at 1.17 (a), 2.37 (b), and 2.45 (c) \% longitudinal strain over the wave, showing damage progression. Strain values correspond to first cohesive element damage (a), ultimate stress (b), and final failure (c).

It should be noted that these strain fields were generated by modeling one half of the coupon and mirroring. This resulted in shear strain values being the same on either side of the wave down the length. However, these strains would be opposite magnitudes. Shear strain starts accumulating within the location of maximum misalignment of the wave as well as in each corner of the coupon. As loading progresses and cohesive elements damage and fail, shear strains become centered within each fiber bundle because the cohesive elements cannot support shear between adjacent bundles. 
The cohesive elements are also included at each of the 3 far-field strain states discussed above, as can be seen in Figure 58.

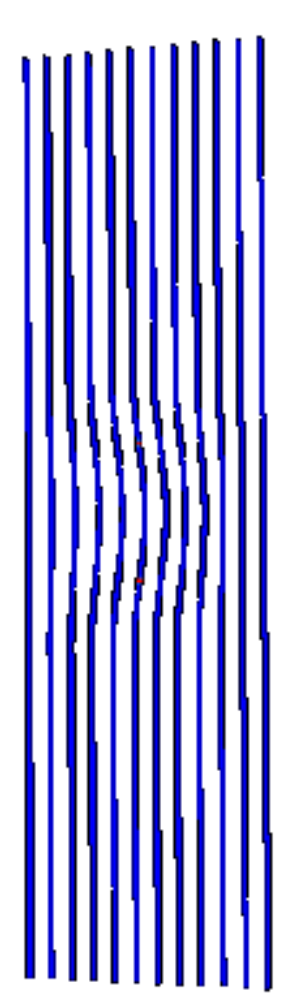

a

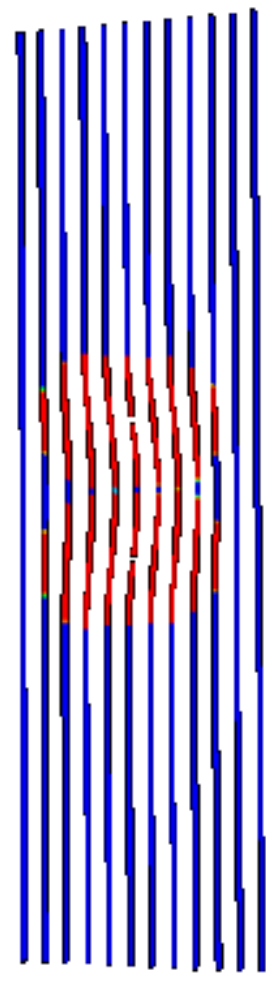

$\mathrm{b}$

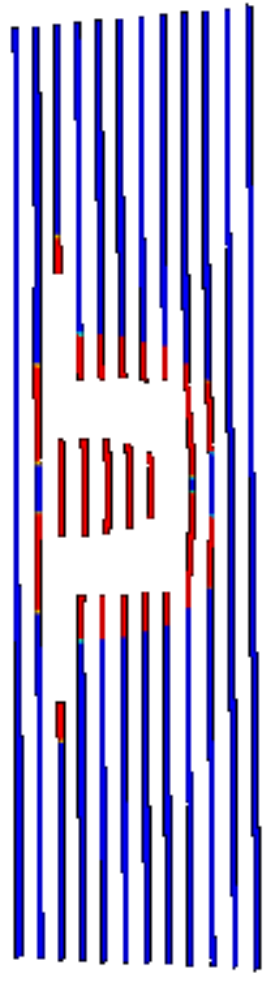

$\mathrm{c}$

Figure 58: Cohesive element damage at 1.17 (a), 2.37 (b), and 2.45 (c) \% longitudinal strain over the wave, showing damage progression. Strain values correspond to first cohesive element damage (a), ultimate stress (b), and final failure (c). Blue elements are undamaged and red elements are damaged.

It can be seen that the first cohesive elements to be damaged are in the location of maximum misalignment angle. As damage progresses, cohesive elements in locations of lower misalignment angles become damaged. The first cohesive element failure occurs in the element that damages first. This occurs just before ultimate stress is reached. Immediately after this failure, cohesive elements in two more columns also fail. These are in the column one to the left as well as fifth from the right, which eventually fails 
through the midline. The damage progression of these cohesive elements gives insight into the shear and transverse strain effects noted in Chapter 3. While the first cohesive element damage and deletion is shear dominated, immediately after, a cohesive element on the right side fails due to a combination of the two stress types. Soon after, this cohesive element column fails through the midline almost entirely due to high transverse stress.

\section{$\underline{\text { Coupon Test and Modeling Results and Comparison }}$}

The CFIP wave coupon model is now compared to the testing. Comparisons are made with stress-strain curves, ultimate stress and strain at failure values, and strain fields. First, stress-strain curves are compared. The model stress-strain curve from Figure 53 above along with the typical experimental stress-strain curve from Figure 36 are shown in Figure 59. 


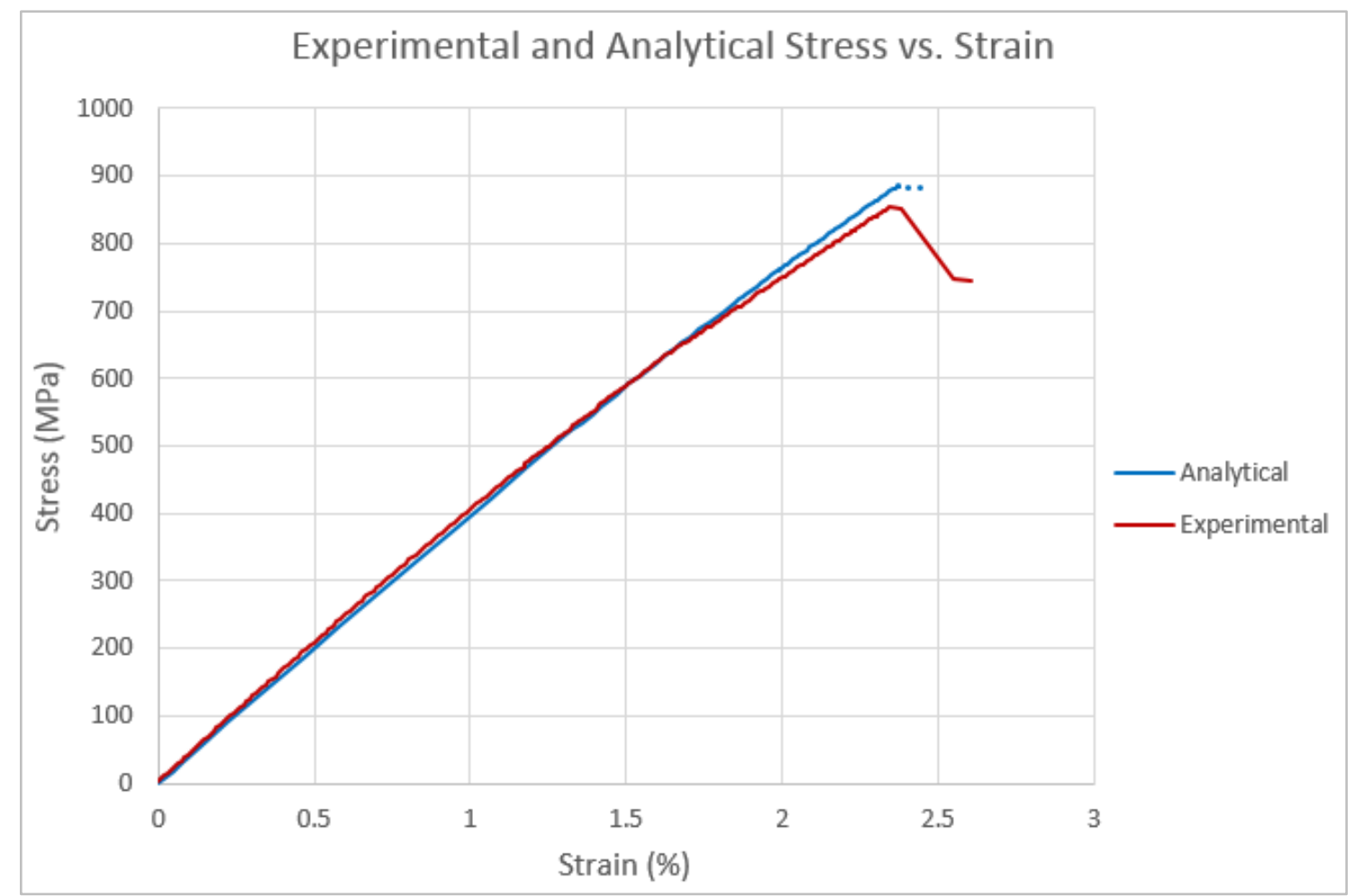

Figure 59: Analytical and Experimental Stress vs. Strain curves showing good analyticalexperimental correlation.

Good correlation is seen between analytical and experimental stress-strain curves. They are nearly linear up to reaching ultimate stress. At this point the stress drops while still increasing strain. After this portion of softening, ultimate failure occurs. Both curves have very similar ultimate stresses. Strain at failure, as well as strain between ultimate stress and final failure are slightly larger for experimental. The experimental curve also loses more stress between ultimate stress and final failure. It can be seen that the experimental curve loses more stiffness before ultimate stress. These curves diverge slightly around $1.75 \%$ strain with the more compliant experimental curve losing stiffness. Altering the nonlinear shear softening UMAT inputs could possibly result in better correlation. However, this was not attempted because the UMAT inputs were chosen as 
notable points in the curve and altering this for better correlation would make the added correlation non-predictive. As mentioned above, the nonlinear shear inputs followed the curve in Figure 9 which did not exhibit negative stiffness due to convergence issues. This was not the composite response but was added stiffness which, if taken into account, could result in even better correlation.

Now, quantitative stress and strain comparisons are made. Analytical ultimate stress is $885 \mathrm{MPa}$ compared to an average experimental ultimate stress of $820 \mathrm{MPa}$. This value is fairly close to the average and is within the 760 to $890 \mathrm{MPa}$ range seen. Analytical strain at failure is $2.37 \%$ compared to an average experimental strain at failure of $2.34 \%$ strain, with a range of 2.11 to $2.51 \%$ strain. These values are very similar, especially for a value with such a large experimental range. The initial elastic moduli for the model is $39.8 \mathrm{GPa}$ compared to an average experimental modulus of $39.2 \mathrm{GPa}$ with a range of 36.7 to $41.3 \mathrm{GPa}$.

Comparisons are now made between strain fields. The DIC images from Figure 28 in Chapter 3 are included again for comparison with the analytical strain fields. These can be seen in seen in Figure 60, Figure 61, and Figure 62. An arrow is included to point to the location of maximum wave amplitude down the length. Both experimental and analytical strain fields are near ultimate stress. The analytical strain fields are at the same steps as the center images in Figure 54 (b), Figure 56 (b), and Figure 57 (b) above but may appear different because the strain scale is changed to match that of the DIC images. The analytical strain fields are also cropped to match the DIC images. 


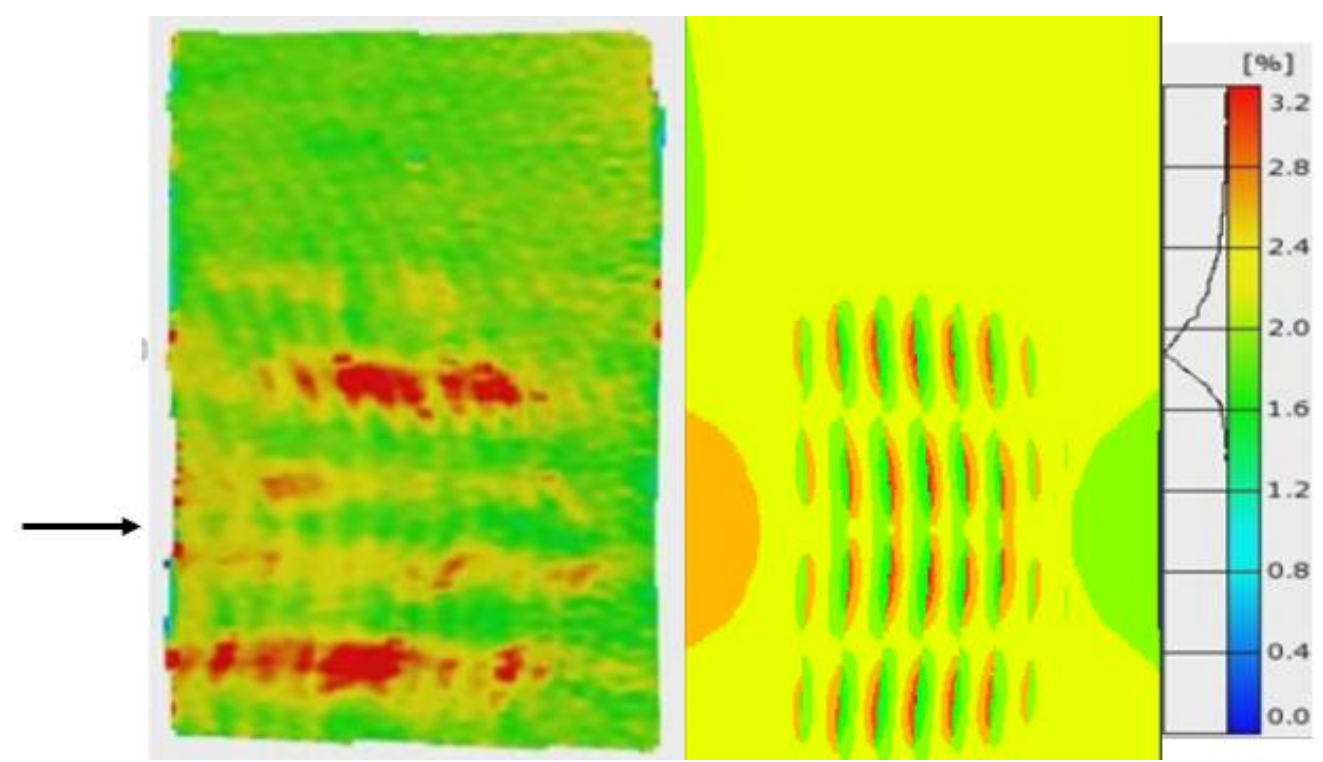

Figure 60: Longitudinal strain field near ultimate stress experimental (left) and analytical (right). Arrow indicates maximum wave amplitude location down length.

Both analytical and experimental longitudinal strain fields correspond well with the exception of the analytical far-field strain being larger than experimental. In both analytical and experimental, higher strain in the wave region can be seen on the left side and lower strain on the right side. They both show the four bands across the width of high strain on either side of the wave. These bands appear more discrete between fiber bundles in the analytical strain field than that of the experimental. Also, the analytical strain field shows all strain bands being of similar magnitudes whereas the experimental shows the two inner bands are of smaller magnitude than the two outer bands. Lastly, the tension and compression causing the bands in the model, as discussed above, is not seen in the experimental strain field. The bands in the experimental strain field are all larger than strain values than the far field value whereas the analytical strain field shows this is not the case. This may be due to the model being constrained within the plane so where 
the compressive bundles during experiment can move out of plane, the $2 \mathrm{D}$ constraint of the model does not allow this, instead, requiring compression of the bundles.

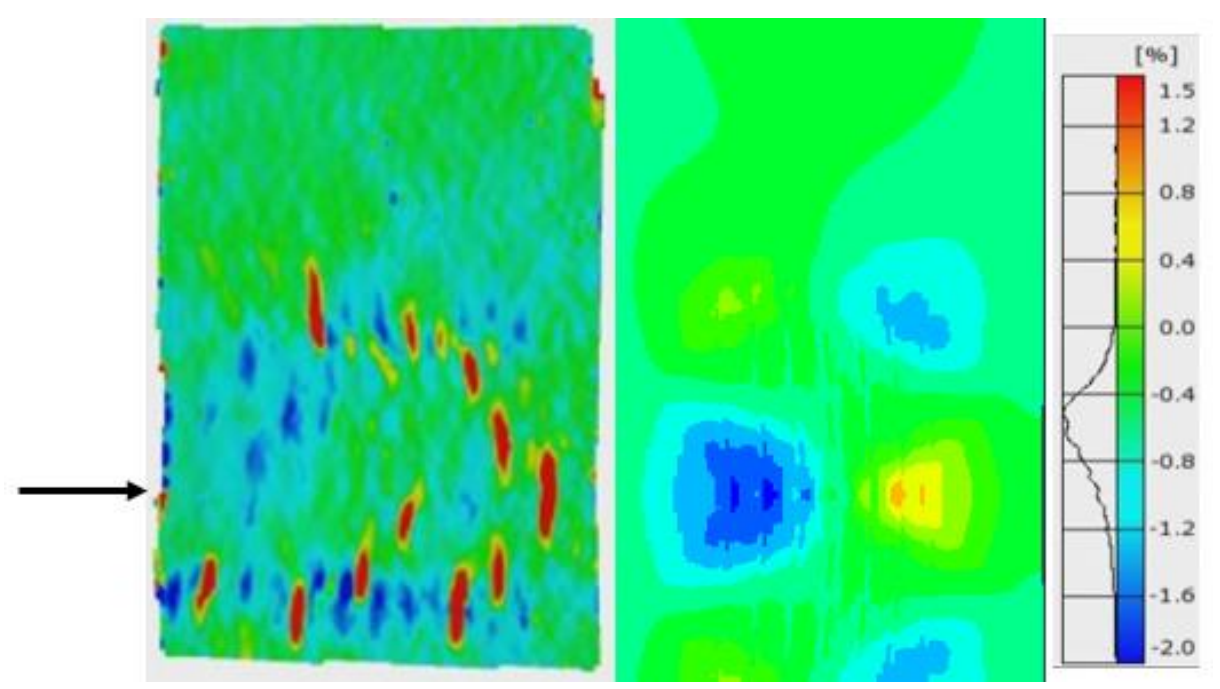

Figure 61: Transverse strain field near ultimate stress experimental (left) and analytical (right).

The transverse strain fields show similar trends. The experimental strain field shows negative strains distributed in $\mathrm{C}$ shape around the wave. The positive strains are distributed, similarly, in a backwards $\mathrm{C}$ shape around the wave. These distributions can also seen in the analytical strain field. The main difference between the two strain fields is that the analytical field has more continuous high and low strain areas whereas the experimental strain field has more discontinuous high and low strain areas. These discontinuous high strain areas in the experimental strain field are also larger magnitude. This is likely due to the DIC images being surface strain distribution. Matrix cracking on the surface ply gives large discontinuous strain values. These are not necessarily indicative of discontinuous through-thickness strains of equally large magnitude. The model assumes homogeneity through the thickness which is why these discontinuities do 
not appear in the model strain field. In the model, discrete, single ply matrix cracks can be considered to be taken into account by the softening portion of the bi-linear traction criteria after damage initiation has occurred.

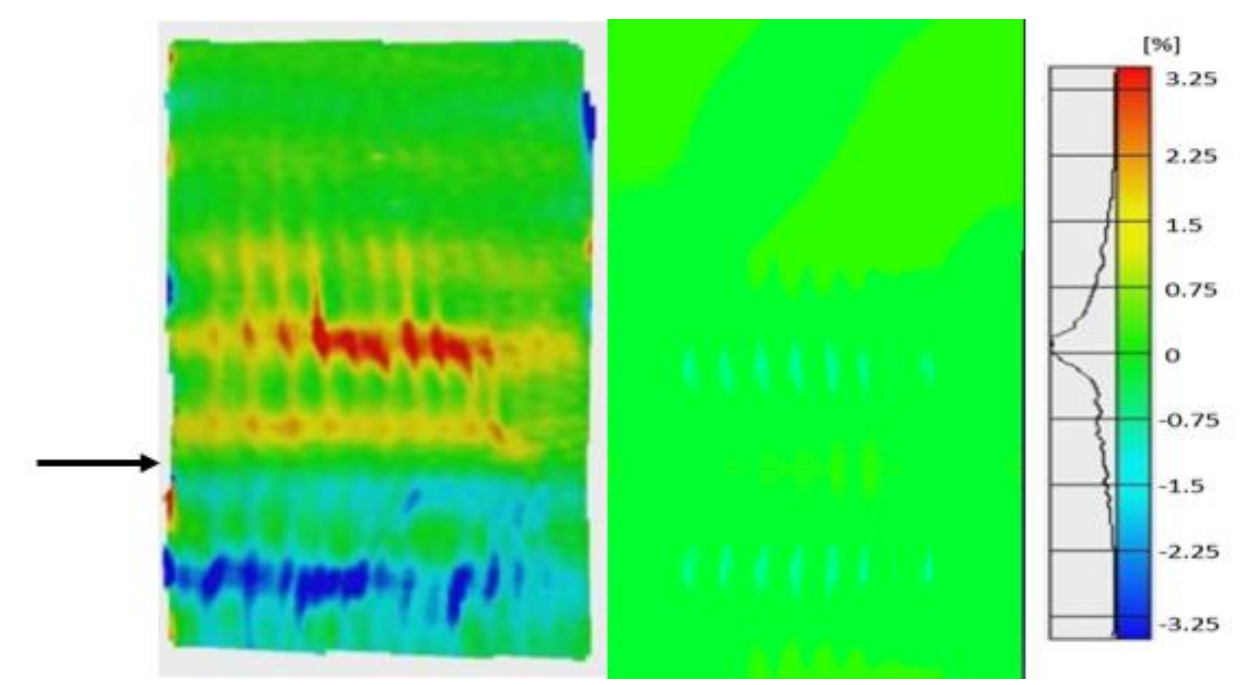

Figure 62: Shear strain field near ultimate stress experimental (left) and analytical (right).

The sign difference between either side of the wave not matching is due to mirroring the analytical strain field over the midline, as discussed above. While there are many discrepancies between experiment and model for the shear strain field, the similarities are discussed first. Due to the difference in magnitude, qualitative comparisons will be made using the experimental strain field here and the model strain field in Figure 57 (b) so that the strain distribution can more easily be seen. Both strain fields show largest magnitudes in the middle across the width. This is shear strain is proportional to the misalignment angle and misalignment angle is greatest in the middle. Both strain fields also show discretization across the width at a spacing equal to that of the fiber bundle width. This is due to loss of load carrying capability of the resin as it damages and is discussed more below. 
Of the strain fields, the shear strain field is the most different between experimental and analytical. The two large differences are the magnitude of shear strain being larger experimentally as well as high shear areas appearing in multiple bands across the width on either side of the wave, rather than one larger band on either side. These discrepancies are now discussed.

As can be seen in Figure 57 (a) above, shear strain magnitude is proportional to the degree of misalignment and, before extensive cohesive element damage, there is no division of shear strain between fiber bundles. Shear strain increases during loading until cohesive elements damage. Damaged cohesive elements soften and the amount of shear stress they transmit between fiber bundles no longer increases with loading. The softening and discretization of shear strain into individual fiber bundles can be seen in Figure 57 (b \& c) compared to Figure 57 (a). The compliance caused by damaged cohesive elements does not allow for shear strains to develop as large as those seen in testing and causes the magnitude discrepancy seen in Figure 62. While there is a loss in stiffness once matrix damages in testing, the overlap between plies in experimental coupons allows for increasing load carrying capability whereas there is no overlap in the model, as discussed above. While it may seem that the *DAMAGE INITIATION parameter should be increased in order to increase stress levels attainable by cohesive elements, this will adversely affect previous correlations.

The other experimental-analytical correlation discrepancy is that 4 bands of high shear strain are seen in testing but only 2 bands are seen analytically. As discussed above, since fiber misalignment causes shear strains, the shear strain distribution would 
be expected to be that seen in the model. The bands seen in the DIC shear strain images were discussed in Chapter 3 and are summarized here. The bands are likely due to the stitching material in the fabric which is why they both have equal spacing, repeating the pattern every $1 \mathrm{~cm}$. So, whereas the DIC image shows discrete bands of high shear strain which fade out away from maximum misalignment angle back to alternating shear bands, the analytical strain field does not show this. This is because the stitching is not taken into account in the model and also because this is likely only an artifact on the surface of the coupon captured by the DIC. This discretization could also play a role in the magnitude difference between experimental and analytical strain fields. The shear strain is binned into discrete bands which can possibly result in constructive and destructive interference between the stitching induced shear and the fiber misalignment induced shear strain.

\section{Effects of Changes between DFIP and CFIP Model}

There were multiple changes between the previous development of this modeling method for the DFIP wave and this iteration for the CFIP wave. The most obvious change is the wave type modeled. Along with this there were changes to the modeling method itself. These changes were:

1. Changing cohesive element material properties

a. *DAMAGE INITIATION based on experimental trends

b. *DAMAGE EVOLUTION based on the quasi 3D calculations

2. Modeling fiber bundles discretely rather than fiber tows

The effects of these changes were investigated and are discussed below. 


\section{Effect of Changing Cohesive Element Properties}

The cohesive element material properties for the iteration of this modeling method for the CFIP wave included herein have been updated from the previous iteration for the DFIP wave. Now that determination of the cohesive element properties for this iteration have been discussed along with the results above, comparisons can be made with these cohesive element properties and the previous properties. *DAMAGE INITIATION inputs were determined so that cohesive elements would damage at the same far-field strain as seen in testing. *DAMAGE EVOLUTION inputs were determined based on maintaining through-thickness fracture energy between experimental coupons with through-thickness complexity and the modeled coupon with no through-thickness complexity. In short, *DAMAGE INITIATION inputs were decreased and the *DAMAGE EVOLUTION inputs were increased. The stress-strain curve for each of models with each of these properties can be seen in Figure 63. "Original" is with the previous cohesive element properties and "Adjusted" is with the new values used herein. A typical experimental curve is also included for comparison. These curves have not been truncated based on a far-field strain. 


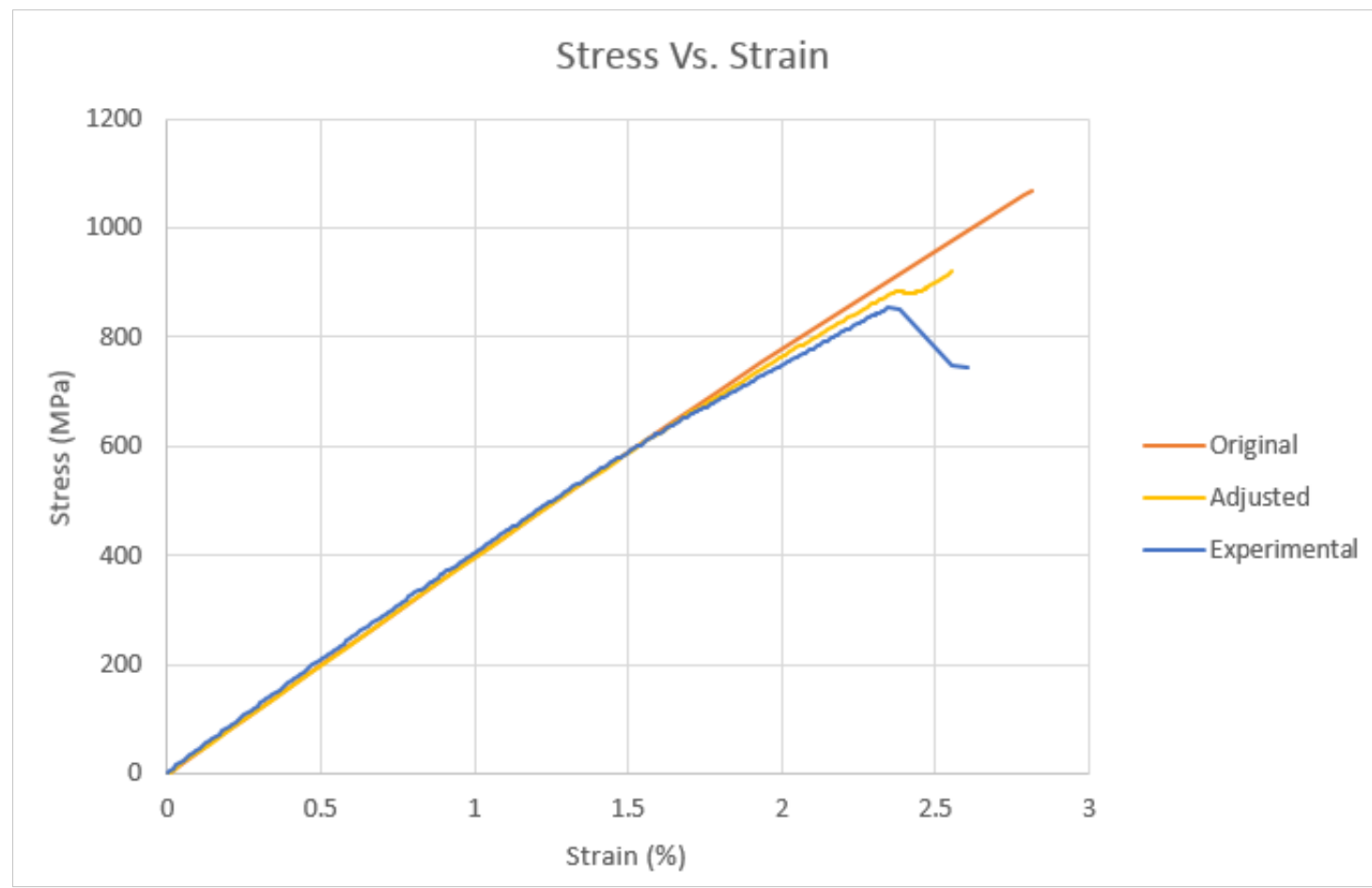

Figure 63: Stress vs. Strain curves for model with original as well as adjusted cohesive element material properties. A typical experimental curve is included for comparison.

From Figure 63, it can be seen that the Adjusted curve loses stiffness around $1.75 \%$ strain and reaches an ultimate stress before losing some load carrying capability, similar to the experimental curve. The Original curve is linear with no visible decrease in load carrying capability. This is because no cohesive elements damaged up to the $2.8 \%$ strain this model was run to. Ultimate stress in experimental coupons was reached around $2.34 \%$ strain and the model with original cohesive element properties does not even have cohesive elements damaged by this strain. Due to no cohesive element damage, strain distributions also do not match experimental. This means that in order to get an accurate response, the $* D A M A G E$ INITIATION inputs needed to be decreased as they have been. Along with decreasing the *DAMAGE INITIATION inputs, *DAMAGE 
EVOLUTION inputs must be increased. This is explained in detail above to account for 3D complexity.

The implication with changing the *DAMAGE INITIATION values to get a wellcorrelated response that results in cohesive element damage should not be overlooked. This value needs to be decreased because the misalignment angles in the wave are smaller in this model than in the DFIP model. Degree of misalignment correlates directly with shear stress which is a criteria for cohesive element damage. This means that either this value was too high in previous model development or this is not a material property of the resin. If the $* D A M A G E$ INITIATION input has to be changed for varying degrees of fiber waviness, this modeling method may not be as predictive as hoped. This would mean that $* D A M A G E$ INITIATION input is not an intrinsic material property of the resin. In order to maintain predictivity with the current bi-linear cohesive traction-separation criteria, there must be an investigation into what *DAMAGE INITIATION inputs are required for a given misalignment angle. The other possibility for predictivity is using the tabular cohesive element traction-separation criteria to input exact response as discussed above.

\section{Effect of Discretely Modeling Fiber Bundles}

As discussed above, the model herein discretely models only fiber bundles which means that cohesive element columns are separated by the fiber bundle distance of $4 \mathrm{~mm}$. Previous development of this modeling method for the DFIP wave coupon modeled fiber tows discretely, which utilized cohesive element columns every $1 \mathrm{~mm}$ across the width. The decision to switch to $4 \mathrm{~mm}$ spacing was based on resin rich areas being visually 
determined to be between bundles. Also, the fiber bundle separation can be seen in the DIC data whereas fiber tow separation is not seen. Due to this, the resin rich areas between fiber tows were considered negligible compared to those between fiber bundles.

To investigate the effect of this change, model runs with both $1 \mathrm{~mm}$ and $4 \mathrm{~mm}$ cohesive element column separation are compared. These are first compared using the original cohesive element properties and then the adjusted values. The stress vs. strain curves for $1 \mathrm{~mm}$ and $4 \mathrm{~mm}$ cohesive separation models can be seen in Figure 64. A typical experimental curve is included for comparison.

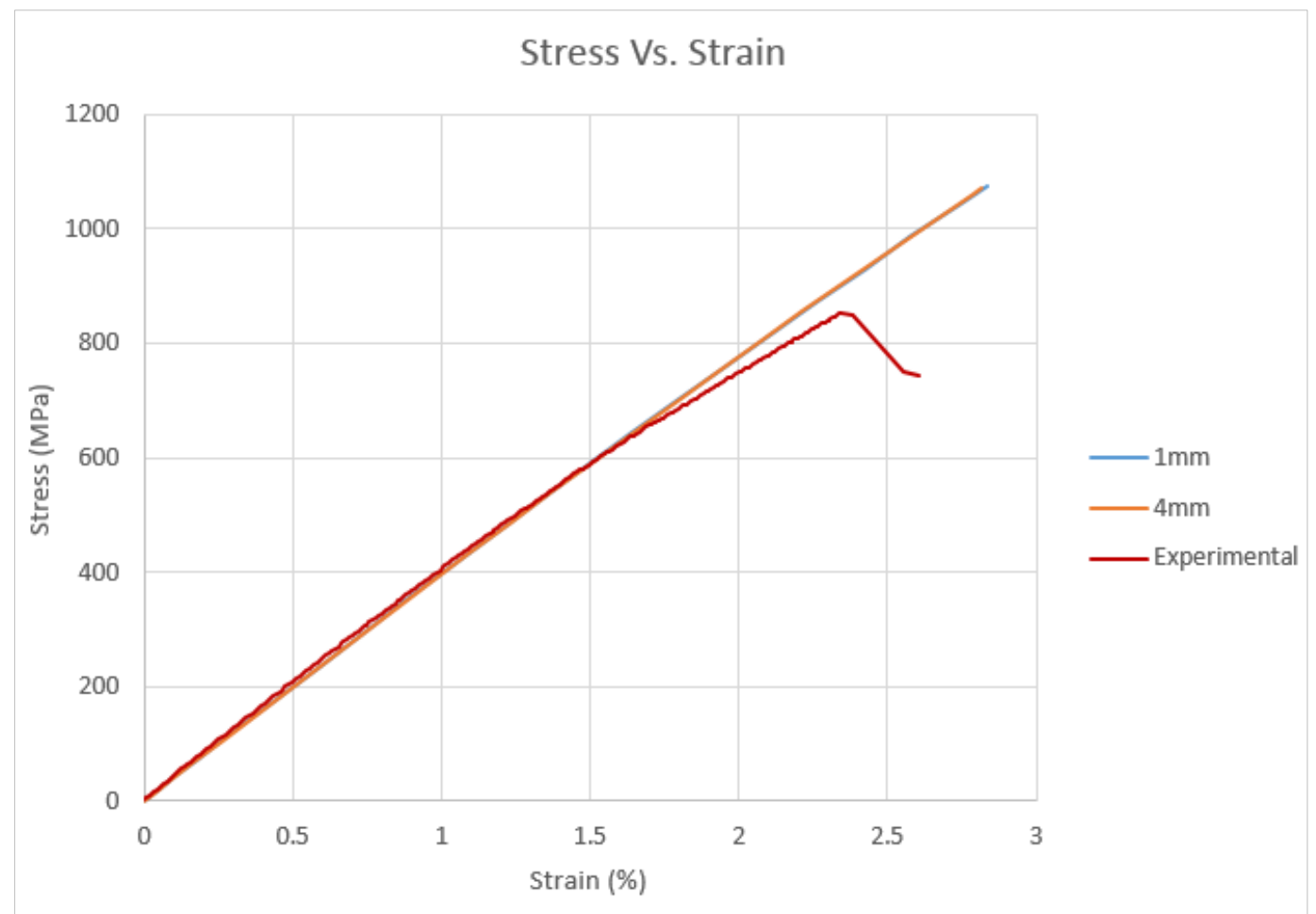

Figure 64: Analytical Stress vs. Strain curves for $1 \mathrm{~mm}$ and $4 \mathrm{~mm}$ cohesive element spacings with original cohesive element properties showing no difference in response. Experimental curve included for comparison. 
The analytical curves for both cohesive element separation distances are the same. Due to the large *DAMAGE INITIATION input, no cohesive element damage has occurred by the point of truncation at $2.8 \%$ strain. For the original cohesive element properties, there is no difference between $1 \mathrm{~mm}$ and $4 \mathrm{~mm}$ cohesive element separation meaning that, as expected, reducing the *DAMAGE INITIATION input was not necessary due to the larger cohesive element spacing. The difference in separation for adjusted cohesive element properties is now investigated.

Model runs were also performed for both the $1 \mathrm{~mm}$ and $4 \mathrm{~mm}$ cohesive separation with updated cohesive element properties. It should be noted that, while $* D A M A G E$ INITIATION values are not affected by cohesive separation, the *DAMAGE EVOLUTION values are affected. As discussed above, the maximum *DAMAGE EVOLUTION values were calculated using maximum overlap between fiber bundles between plies. The maximum overlap is half the bundle width. For the $4 \mathrm{~mm}$ cohesive separation, the maximum overlap is $2 \mathrm{~mm}$. For the $1 \mathrm{~mm}$ cohesive separation, the maximum overlap is $0.5 \mathrm{~mm}$. These calculations using Equations 2 through 9 were performed with $0.5 \mathrm{~mm}$ maximum overlap. This resulted in Mode I and Mode II effective critical energy release rates of $1.378 \frac{\mathrm{J}}{\mathrm{m}^{2}}$ and $2.096 \frac{\mathrm{J}}{\mathrm{m}^{2}}$ respectively. These can be compared to $3.092 \frac{\mathrm{J}}{\mathrm{m}^{2}}$ and $3.810 \frac{\mathrm{J}}{\mathrm{m}^{2}}$ respectively for the $4 \mathrm{~mm}$ cohesive separation as calculated above. The stress-strain curves for both the $1 \mathrm{~mm}$ and $4 \mathrm{~mm}$ cohesive separation models with the adjusted *DAMAGE INITIATION value of $60 \mathrm{MPa}$ and their respective *DAMAGE EVOLUTION values can be seen in Figure 65. Also included in this plot is a typical experimental curve. 


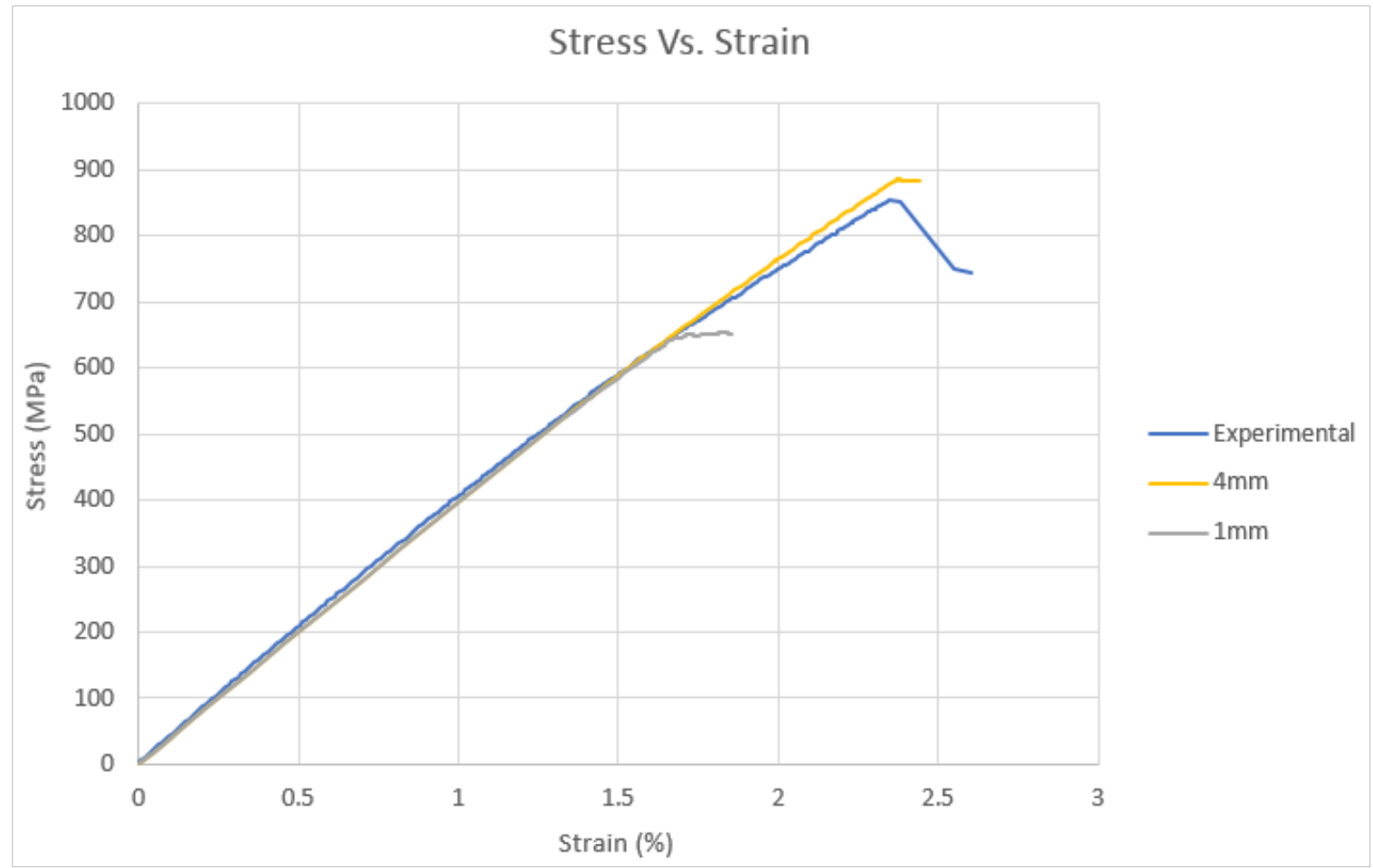

Figure 65: Analytical Stress vs. Strain curves for $1 \mathrm{~mm}$ and $4 \mathrm{~mm}$ cohesive element spacing with adjusted cohesive element properties. Experimental curve is included for comparison.

Due to the analytical curves having an obvious ultimate stress and final failure, as discussed above, truncation has been applied to these curves. It can be seen that for these curves, when the $* D A M A G E$ INITIATION stress has been reached, there is a difference in response between $1 \mathrm{~mm}$ and $4 \mathrm{~mm}$ cohesive element separation. The $1 \mathrm{~mm}$ cohesive separation model softens and fails soon after cohesive element damage whereas the $4 \mathrm{~mm}$ cohesive separation model softens slowly and has a response more similar to experimental. The cause of the premature failure in the $1 \mathrm{~mm}$ cohesive separation model is the lower *DAMAGE EVOLUTION values. Given that damage initiates in the cohesive elements at the same stress in both models, a lower *DAMAGE EVOLUTION value results in earlier failure due to faster crack propagation. As mentioned above, the 
*DAMAGE EVOLUTION values were reduced due to tow-tow overlap between plies being smaller than bundle-bundle due to the smaller width. This reduced the energy for crack propagation. Given that the $4 \mathrm{~mm}$ separation model correlated much better to experimental than $1 \mathrm{~mm}$ separation, this further supports that cracks propagate between bundles in experimental coupons rather than between tows.

An interesting finding upon investigation of the strain field of the $1 \mathrm{~mm}$ cohesive separation model is the separation in high strain locations. Similar to the DIC images, which sparked the change in cohesive separation, it can be seen that the high strain locations are separated by $4 \mathrm{~mm}$ still. Figure 66 is an image of the longitudinal strain field over the wave of this model at ultimate stress. Distances between high strain locations are labeled.

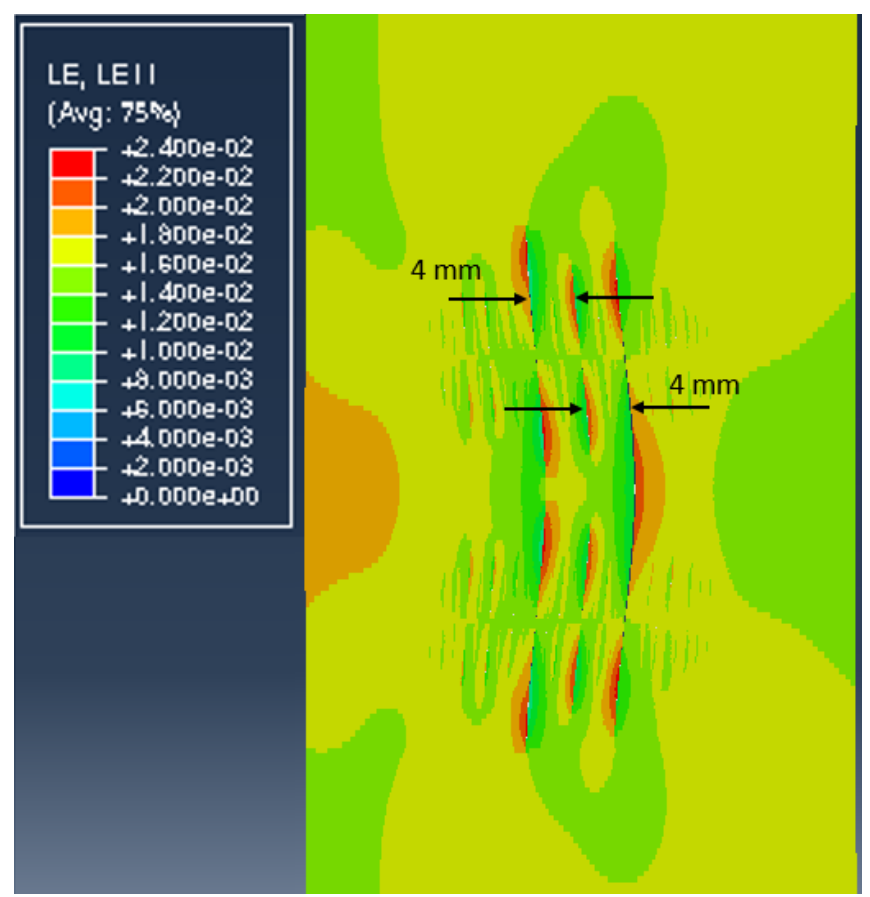

Figure 66: Longitudinal strain field over wave section of model with $1 \mathrm{~mm}$ cohesive element spacing with adjusted cohesive element properties. Spacing between high strain regions can be seen and labeled as $4 \mathrm{~mm}$ spacing despite cohesive element spacing. 
From Figure 66 it can be seen that the distance between the highest strain locations is $4 \mathrm{~mm}$, the same distance seen in the DIC images. There are also lower strain concentration regions which are separated by $1 \mathrm{~mm}$, the cohesive element separation distance. This is an important note because perhaps the strains are not concentrated enough to cause large cohesive element damage at every cohesive element column. When one cohesive element column is damaged then the surrounding strains are relaxed which makes it so that nearby cohesive elements do not damage as severely. This could be the same mechanism causing the $4 \mathrm{~mm}$ separation seen in testing even though fiber tows are only $1 \mathrm{~mm}$ wide.

The last portion of this investigation of cohesive element spacing is the effect on model processing time. Previous work on this modeling method has been successful but has also stated that reducing processing time is an important step forward (Nelson, 2013). As seen here in Figure 65 as well as all of the discussion above, using a 4 mm cohesive element separation to discretely model only fiber bundles is an accurate modeling method. The importance of this is the effect on processing time. First, the CFIP models with $1 \mathrm{~mm}$ cohesive separation and $4 \mathrm{~mm}$ separation are compared. Second, the CFIP 4 mm separation model is compared to the DFIP $1 \mathrm{~mm}$ separation model.

The CFIP model with $4 \mathrm{~mm}$ separation compared to the CFIP model with $1 \mathrm{~mm}$ separation uses about a quarter of the cohesive elements. The $4 \mathrm{~mm}$ separation model uses 1920 cohesive elements while the $1 \mathrm{~mm}$ separation model uses 7840 . The cohesive element separation does not affect the number of bulk elements which is 16,000 for both models. As seen in the model runs here with original cohesive properties, this reduces 
processing times from 5 minutes for the $1 \mathrm{~mm}$ spacing to 2 minutes for the $4 \mathrm{~mm}$ spacing. The run times are short due to cohesive elements not damaging but this is still a $60 \%$ decrease in processing time. For adjusted cohesive properties, the $1 \mathrm{~mm}$ spacing processing time is over 60 minutes whereas for the $4 \mathrm{~mm}$ spacing, the processing time is just over 35 minutes. This is a $40 \%$ reduction in processing time. While not as large of a percentage decrease is seen when cohesive elements damage, this is still a significant decrease in processing time and this results in better experimental correlation.

The difference in processing times between the DFIP and the CFIP models, both with $1 \mathrm{~mm}$ cohesive element spacing, is now discussed. Model runs for the DFIP model took up to 240 minutes (Nelson, 2013) whereas the CFIP model run, even with the same cohesive element spacing, takes 60 minutes. This is because there is less cohesive element damage and deletion before failure. This is due to both the continuous fiber IP wave type and the smaller misalignment angle. It should be kept in mind that this reduction is even with the CFIP model containing $60 \%$ more bulk and cohesive elements due to the larger length of $80 \mathrm{~mm}$ per half compared to $50 \mathrm{~mm}$. This processing time reduction of $75 \%$ is notable. Due to CFIP waves being more typical of waves seen in manufactured parts, this reduced processing time will be taken advantage of when modeling waves as seen in parts.

Overall, a $75 \%$ reduction in processing time is due to the less misaligned, continuous fiber wave type and a further $40 \%$ is due to the larger cohesive element spacing. This results in an overall reduction in processing time of $85 \%$ from the $1 \mathrm{~mm}$ spacing DFIP model to the $4 \mathrm{~mm}$ spacing CFIP model. 


\section{$\underline{\text { Modeling Conclusion }}$}

The model further developed herein uses a method combining both continuum and discrete modeling approaches, allowing resin crack propagation to be modeled discretely within a continuum model. The material properties used were determined from previous testing and from parametric studies as discussed above. This model was for a CFIP wave which starts bridging the gap to partial flaw research as well as improve robustness of this modeling method.

The model overall correlated very well with testing. The stress-strain curve shape as well as stress and strain values were very similar. The strain fields also correlated well except for the shear strain field, as discussed above.

Along with checking analytical-experimental correlation, the failure mechanics of this wave type were further investigated as well as the effect of the updates implemented in the model herein. Investigation of cohesive element progressive damage found that certain cohesive elements damage and fail due to a combination of shear and transverse stresses. This is notable because cohesive element damage and failure was shear dominated in the previous DFIP wave. The wave severity gradient across the width introduces more complex interactions which is of importance because this may introduce the necessity of another critical flaw parameter for IP waves of varying misalignment. This is left as future work.

Investigation of cohesive element separation found that, given the method of accounting for through-thickness ply misalignment, as discussed in detail above, the 4 $\mathrm{mm}$ cohesive element spacing results in much better experimental correlation than the 1 
$\mathrm{mm}$ cohesive element spacing. The implication of this is that cracks propagate between the $4 \mathrm{~mm}$ fiber bundles rather than the $1 \mathrm{~mm}$ fiber tows.

Future work that could increase the accuracy of the model is related to the cohesive element material properties. The bi-linear traction-separation criterion can be changed to a tabular criterion which could directly capture the traction-separation response seen above in the model for Mode I separation energy. A similar model could be made in 3D to get a Mode II separation energy and this traction-separation response could also be entered in using the tabular traction-separation criterion. The Mode I and Mode II traction-separation responses could potentially be obtained more accurately experimentally. 


\section{CHAPTER 5}

\section{SANDWICH BEAM DEVELOPMENT}

\section{$\underline{\text { Motivation and Overview }}$}

In order to build upon the extensive effects of defects research on the coupon level, defects in structures, in particular sandwich beams, are investigated herein. The goal of testing sandwich beams in four-point bending is twofold: 1) get more applicable compression results than obtained through coupon testing and 2) compare the effect of fiber waviness in tension and compression in flexural loading with a stabilizing balsa core to that in uniaxial coupon tests. The first goal is important because coupon compression data is conservative when applied to structures. Coupons in compression are more likely to buckle than sandwich beam face sheets in compression because they are not stabilized by being adhered to a structure. Also, other compressive failure mechanisms such as fiber failure or matrix failure tend to occur at larger loads than those for buckling. Therefore, compression data used for designing structures should come from structures so that they are not greatly overdesigned. The second goal is to build a bridge between the extensive coupon-scale research previously performed and the fullscale component scale. This building-block process of increasing complexity can be seen in the testing pyramid above in Figure 2. It is reiterated that the sandwich beam goal for the work herein is to develop the sandwich beam for future testing, not to carry out extensive effects of defects testing on the structure-scale. 
The four-point bend tests were performed in MSUCG's subscale facility shown in Figure 67. This includes four 2.5 inch $(6.35 \mathrm{~cm})$ diameter rollers used as force applicators. All load rollers have adjustable positions along the length. The top load rollers can be set every 5 inches $(12.7 \mathrm{~cm})$ between 10 inches $(25.4 \mathrm{~cm})$ and 30 inches $(76.2 \mathrm{~cm})$. The bottom load rollers can be set at any increment between 30 inches $(76.2$ $\mathrm{cm})$ and 88 inches $(223.5 \mathrm{~cm})$. The upper load rollers are attached to a $52 \mathrm{kip}(231 \mathrm{kN})$ actuator with a maximum displacement of 10 inches $(25.4 \mathrm{~cm})$. The maximum vertical distance between the top and bottom load rollers is 7.75 inches $(20 \mathrm{~cm})$ and when fully displaced, the top load rollers are 2.25 inches $(5.7 \mathrm{~cm})$ below the bottom load rollers.

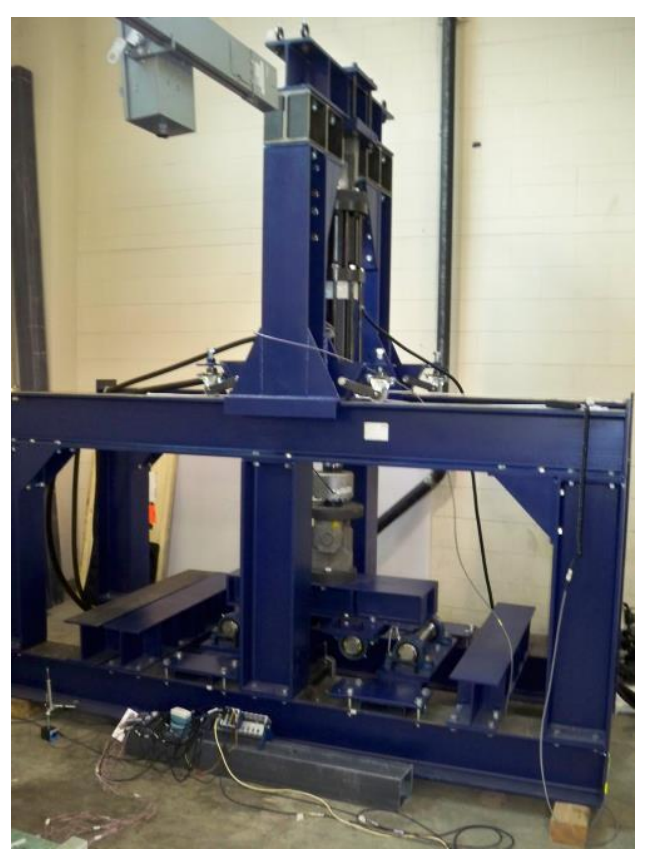

Figure 67: Subscale test frame with actuator and load rollers.

In order to achieve both goals, both compressive and tensile failures must be possible. For a symmetric sandwich beam (i.e. top and bottom face sheets are identical in material and geometry), if a face sheet is to fail, it will be the compressive side face sheet. 
This is because the composite used here is weaker in compression than tension. Strains at failure for this material are about $2.5 \%$ in tension and $1.5 \%$ in compression. Due to the tensile failure requiring a larger strain to failure, this is a more difficult failure to achieve. Therefore, tensile failures were attempted in the design process with the assumption that if a tensile failure could be achieve, a compressive failure would also be possible with minor changes.

Due to a symmetric beam resulting in a compressive failure, an asymmetric sandwich beam was necessary for a tensile failure. This allowed the compression side face sheet to be thicker than the tension side face sheet. Assuming a tensile failure could be achieved, a compressive failure could then be achieved by using the same sandwich beam but loading it into the test facility upside-down so that the tension side face sheet was thicker. For a sandwich beam loaded in 4-point bending as shown in Figure 68, the top face sheet is in compression and the bottom face sheet is in tension.

In order to design the sandwich beam, the mechanics of a four-point bend test must be investigated. The shear and moment diagrams are shown in Figure 68. This shows that the shear in the beam is highest between the top and bottom rollers on either side and zero in the gage section. The moment increases linearly from either bottom roller to a maximum at the inner rollers which remains constant through the gage section. The maximum shear is a function of load while the maximum moment is a function of load and load roller location. Based on this, there is a primary failure mode for both shear and moment. If the beam fails in shear, it will be outside the gage section. If the 
beam fails due to the moment it will be in the gage section. Shear failures are attempted to be avoided.

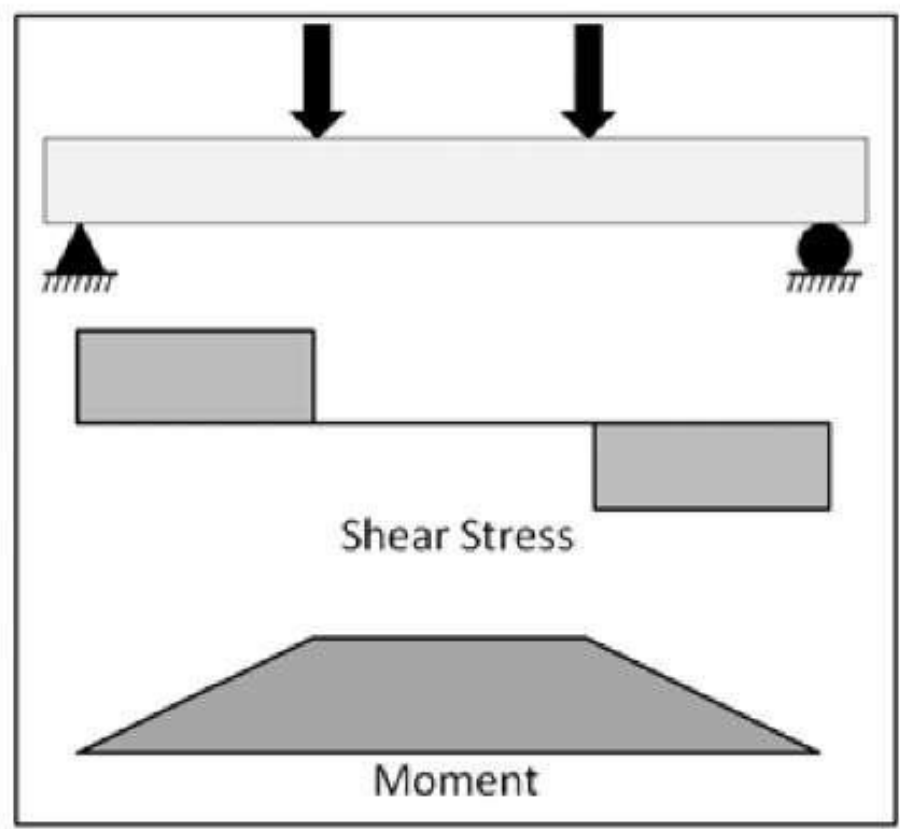

Figure 68: Four-point bending diagram showing shear stress and moment distribution along the length (what-when-how, 2014).

The materials used in the sandwich beam are the same composite materials from above, PPG 1250 glass, 135 RIMR, 1366 RIMH, along with a balsa core. The balsa was 1 inch $(25.4 \mathrm{~mm})$ thick while the face sheet thicknesses could be determined. The width was limited by the mold which was either 5.5 inches $(14 \mathrm{~cm})$ or 8 inches $(20 \mathrm{~cm})$ depending on the mold used as discussed below.

The relevant material properties for strain calculations are both the composite and balsa elastic moduli. To determine when and where failure would occur as well as failure type, face sheet and balsa tensile, compressive, and shear strengths must be known. It was assumed that failure would be due to either face sheet compressive or tensile failure 
or balsa shearing. Delamination between the face sheets and core is a common type of failure for sandwich beams but is assumed not to happen here and, as seen in testing, did not occur for the sandwich beams developed in this work. The elastic moduli of the materials are $40 \mathrm{GPa}$, for the face sheets and 3.2 GPa for the balsa core. The face sheet failure calculations use strain to determine when failure will occur rather than stress. The shear strength for balsa was originally taken to be $3 \mathrm{MPa}$. However, balsa material properties vary depending on batch and environmental conditions. Also, balsa is not homogeneous which can be seen in the grain orientation in Figure 69. The grain orientation is not consistent which means that the properties are location dependent. Given this, the balsa material properties are approximations used for calculations. The segmented balsa in Figure 69 (top) was used for the first 3 sandwich beams and the sheet balsa sheet (bottom) was used for the beams that follow. Due to core shear failures not occurring between balsa segments, the change in balsa is considered negligible.

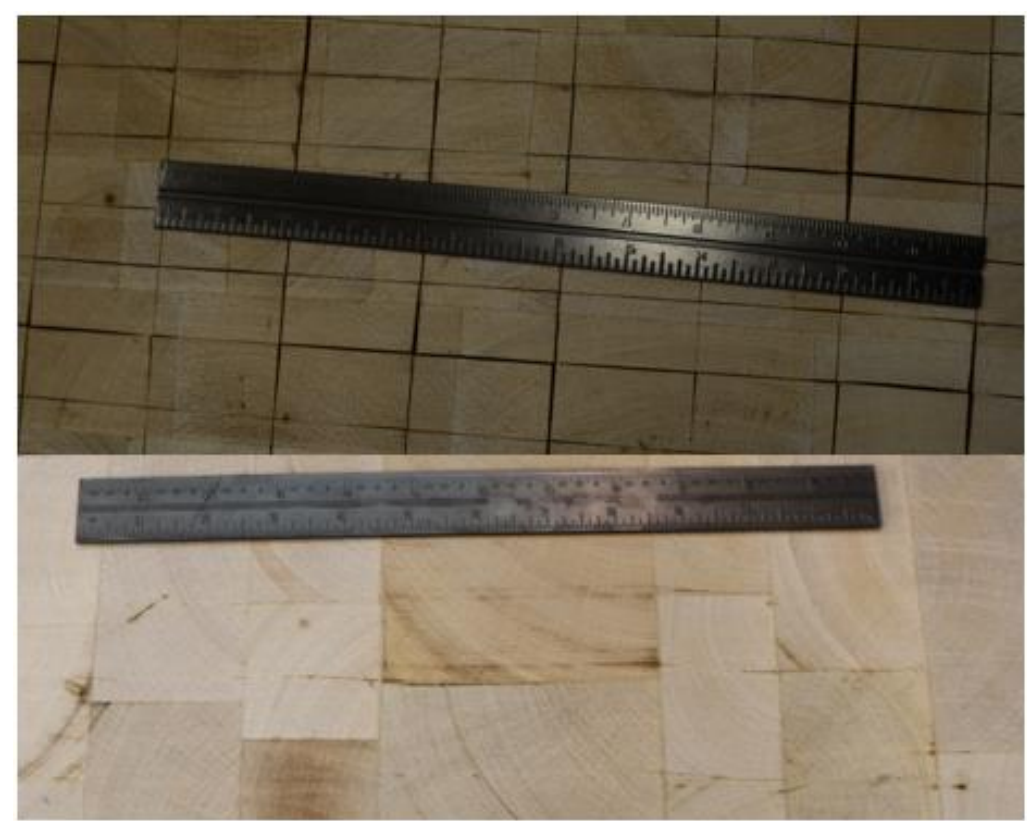

Figure 69: Segmented balsa (top) and sheet balsa (bottom) with 1 foot ruler for scale. 
The given parameters for the sandwich beam design have been listed above. The variables that can be changed are now discussed. In this design the thicknesses of each face sheet, beam width within a range, and load roller locations can be changed. Increasing the distance between the bottom and top load roller increases the moment in the gage compared to the shear outside the gage which will make face sheet failure more likely than balsa shear failure. However, increasing this distance, as discussed below, increases the deflection which must be kept within the actuator's 10 inch $(25.4 \mathrm{~cm})$ range. This design requires balancing multiple variables and is completed through testing and analysis of multiple beam design iterations. These are discussed below.

\section{Design Iteration 1}

The first beam design was based on research performed in parallel within MSUCG. This research used a C-channel mold to make C-channel composite beams. The C-channel mold can be seen in Figure 70 (left) and a composite C-channel made using this mold can be seen in Figure 70 (right). 

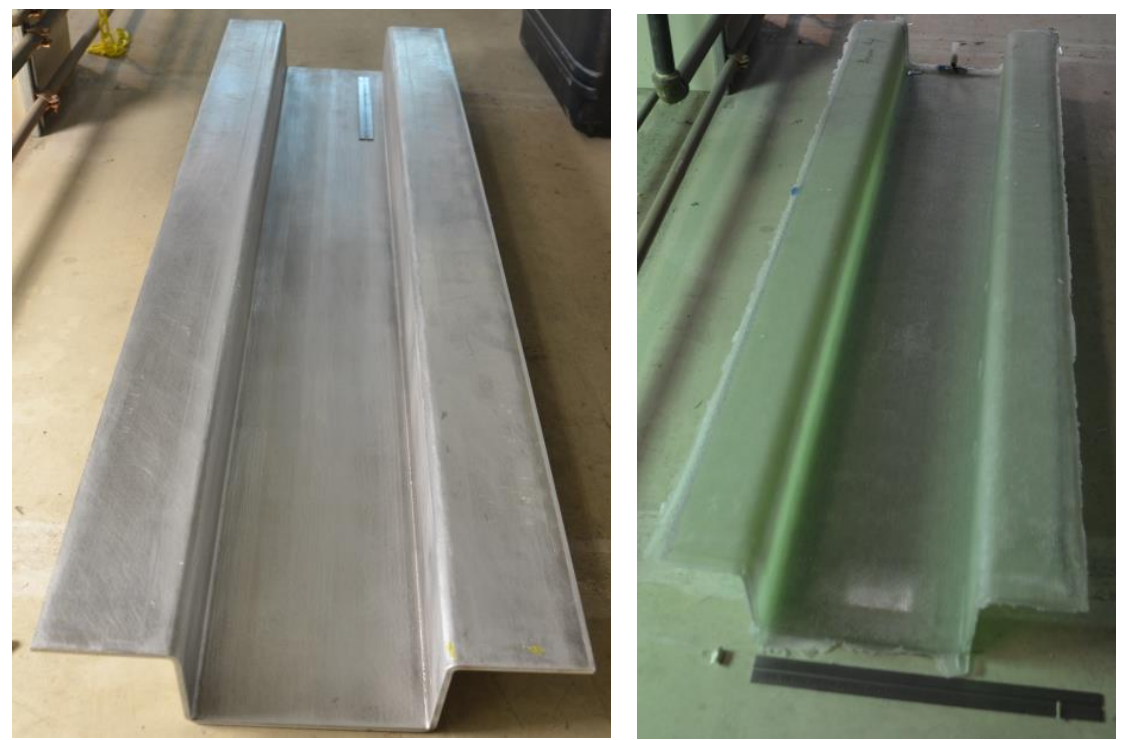

Figure 70: C-channel mold (left) and C-channel composite beam made on mold.

The first beam was designed to use the C-channel face as the tensile face sheet of the sandwich beam. This C-channel beam was 40 inches $(102 \mathrm{~cm})$ long and 4 plies thick and included the radius in the corners. In order for the compression side to not fail first, the compression face sheet was made 8 plies thick. This face sheet was made on the same mold but only over the C-channel face, not extending up the walls. The face sheets were bonded to either side of the balsa core. A cross-section of the completed sandwich beam is shown in Figure 71. 


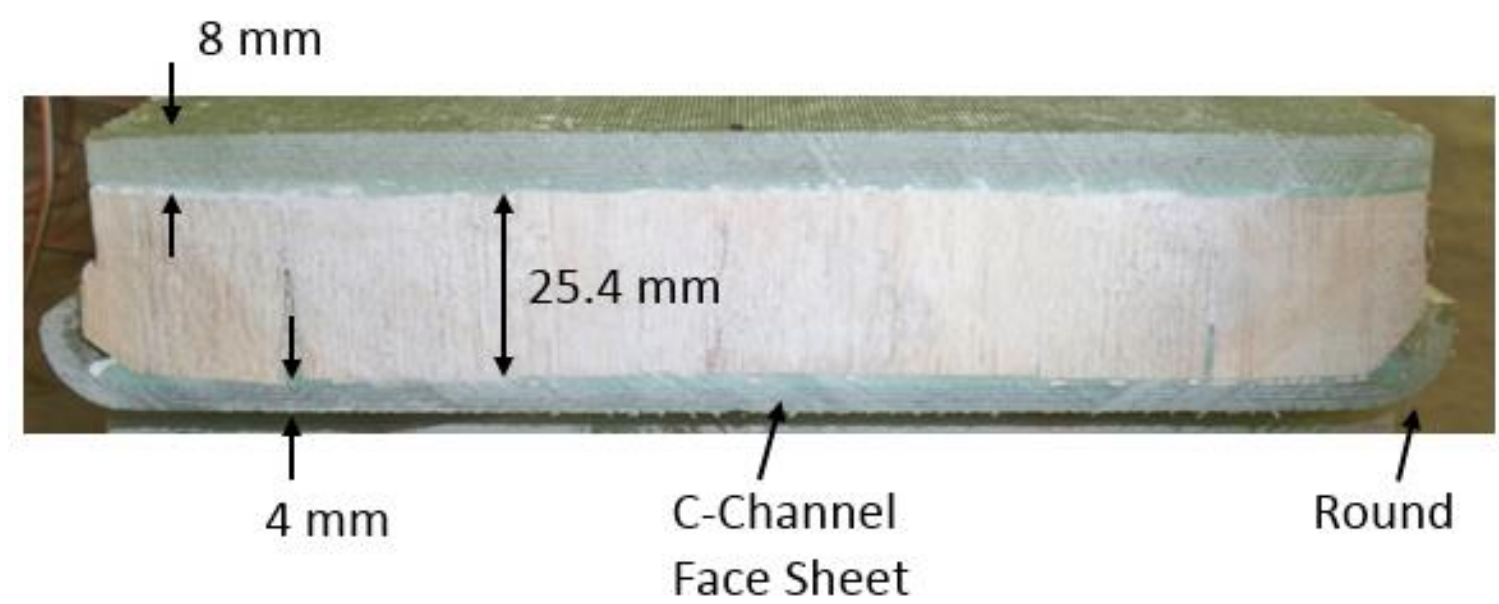

Figure 71: Sandwich Beam 1 looking down the length to show cross-section.

The beam itself was 40 inches $(102 \mathrm{~cm})$ long and due to required overhang past either bottom load nose, the bottom load noses were set to be 36 inches $(91 \mathrm{~cm})$ apart so that there could be 2 inches $(5 \mathrm{~cm})$ overhang on either side. This beam and load rollers can be seen in Figure 73 below. Typical span-to-depth ratios are 16:1 but for strong, orthotropic laminates, this is recommended to be 32:1 up to 60:1 (ASTM D6727). The depth of this sandwich beam was about 1.5 inches $(4 \mathrm{~cm})$ which gives a span-to-depth ratio of 24:1 which was slightly lower than recommended.

The standard loading locations for the top rollers in 4-point bending are typically one-third the bottom load roller distance which, for this setup, would be 12 inches (30 $\mathrm{cm})$. Given the possible locations for top rollers, the top roller distance was set to 15 inches $(38 \mathrm{~cm})$.

Due to possible indentation and associated failure of the face sheets from load application, load pads were used. These were made of $2.5 \times 2.5$ inch $(6.4 \times 6.4 \mathrm{~cm})$ crosssection G10 fiberglass blocks cut in length equal to the width of the beam. G10 fiberglass is a strong, off-the-shelf glass-epoxy laminate. These were placed on the beam 
at all four load application locations and can be seen on both the tension and compression sides in Figure 72 below.

The sandwich beam was instrumented with 6 strain gages and an LVDT. Five of the strain gages were on the tension side face sheet and one was on the compression side face sheet. For the tensile strain gages, one was in the very center of the gage while the other four were put in each quadrant formed by the longitudinal and transverse midlines. The multitude of strain gages was used to check for torsion which was found to be negligible. The last strain gage was in the center of the compression side face sheet. Both the tension and compression face sheet strain gage configurations can be seen in Figure 72. The LVDT was placed in the center of the tensile face sheet gage section to measure maximum beam displacement. The actuator displacement was output with a built-in LVDT. Load data was also output from the actuator.
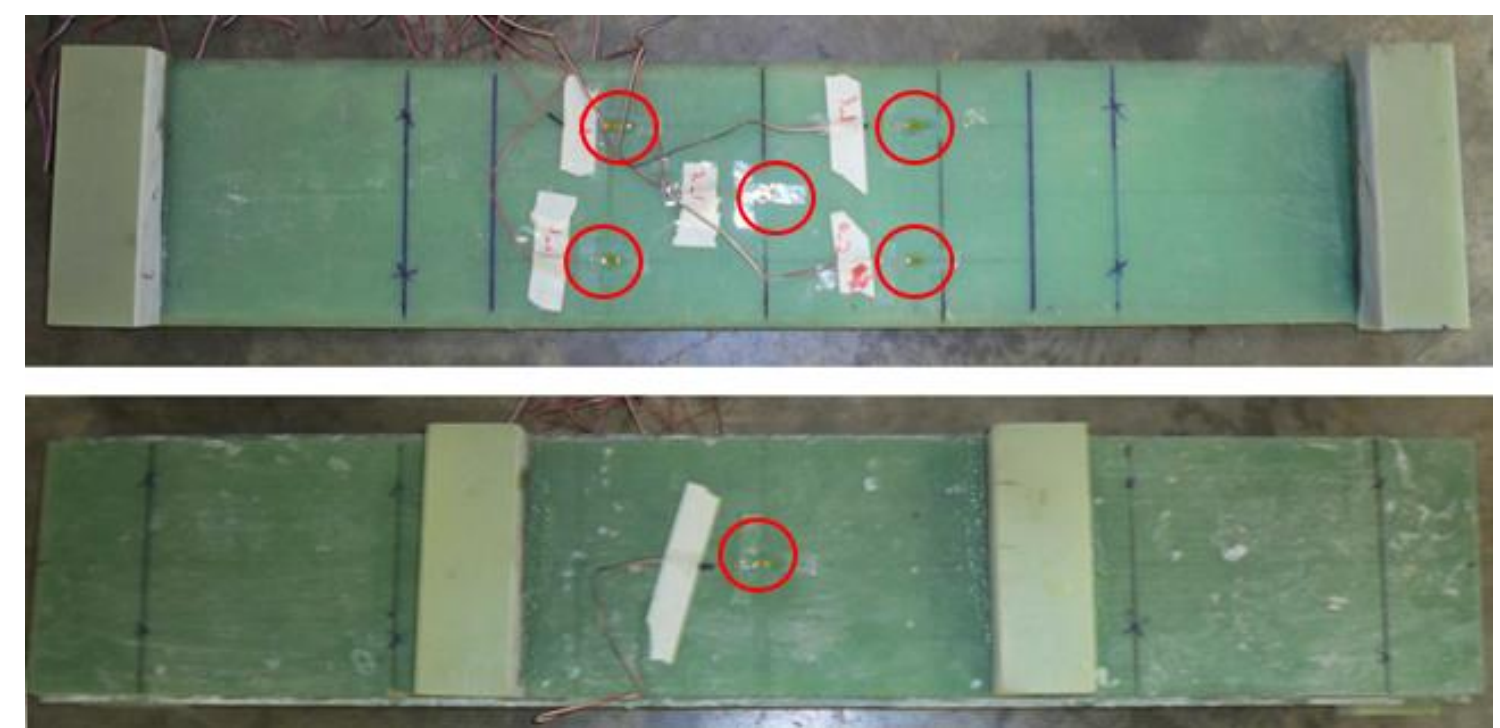

Figure 72: Beam 1 tension side face sheet (top) and compression side face sheet (bottom) showing load pad locations and strain gage configurations circled in red. 


\section{Beam 1 Results}

Beam 1 in the test frame is shown in Figure 73. During testing it was found that the load pads were too small, resulting in the load noses rolling off the load pads at just over $11.1 \mathrm{kN}$, before reaching final failure. After increasing the size of the load pads, the beam was tested to failure at $13.4 \mathrm{kN}$. After investigation, the failure was due to balsa crushing. The Strain vs. Load curves for top and bottom face sheets for the first test are shown in Figure 74. A curve for the second test to failure is not included because the beam was already damaged when starting the second test. While the tension side face sheet strain needs to be $2.5 \%$ strain for failure, only $0.18 \%$ strain was reached at an actuator displacement of $1.0 \mathrm{~cm}$.

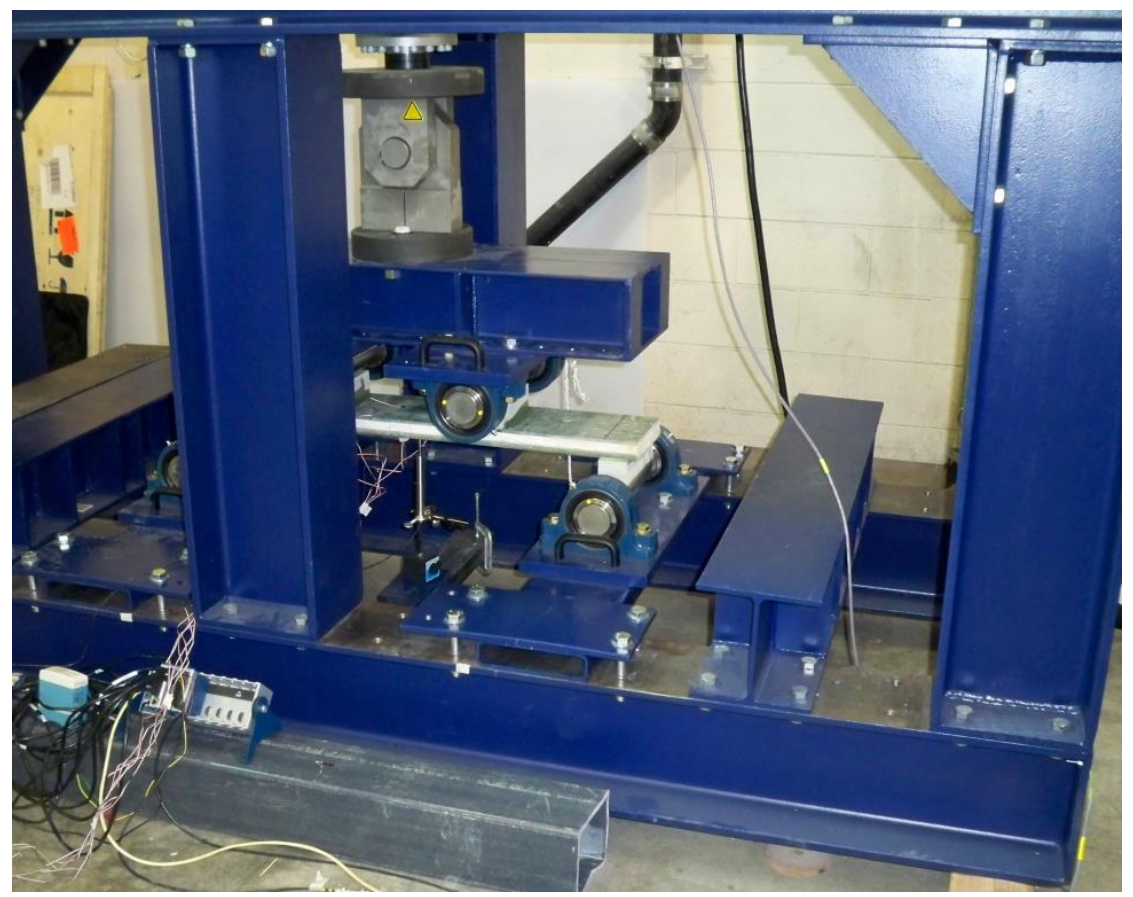

Figure 73: Beam 1 loaded in test frame. 


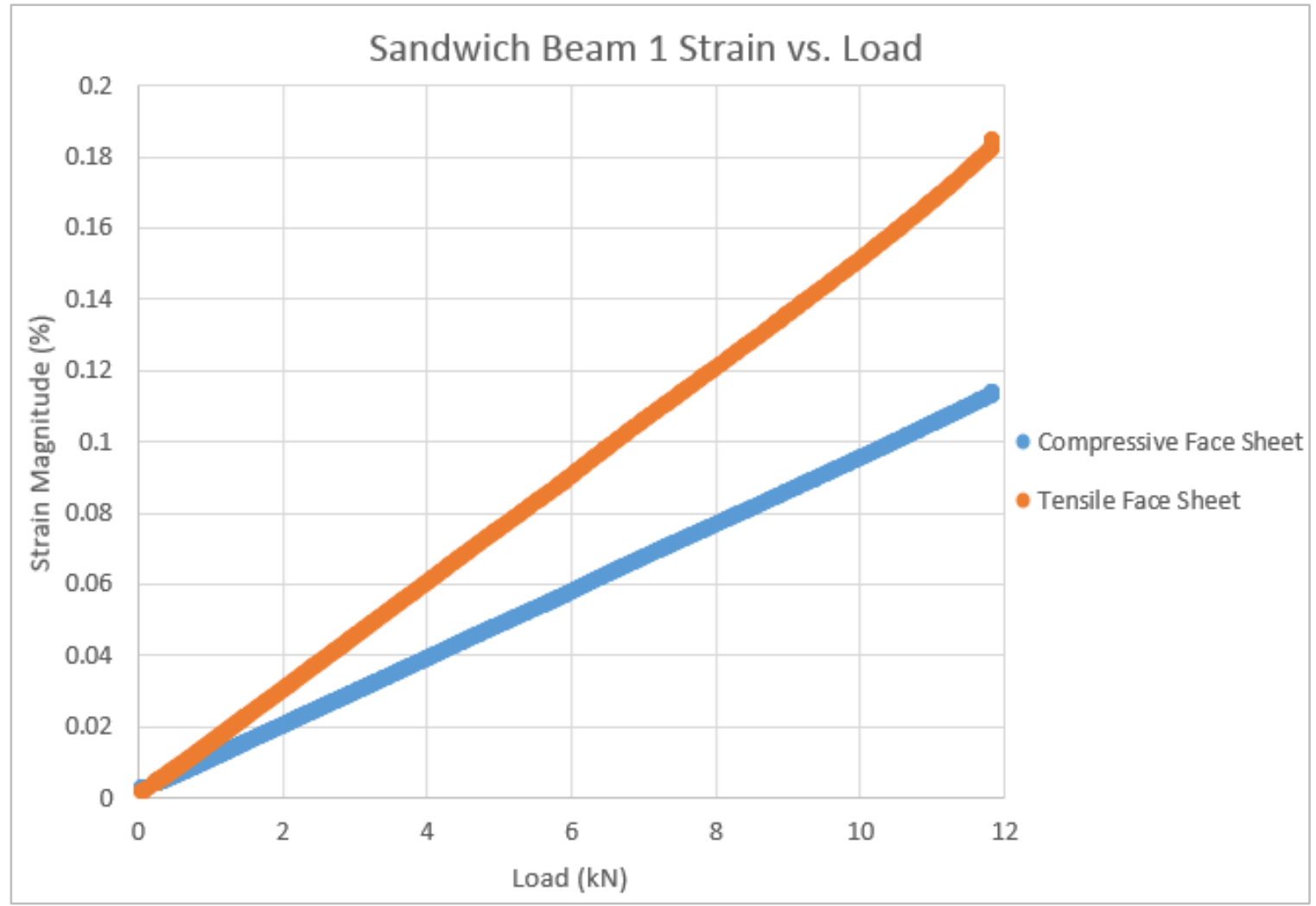

Figure 74: Strain vs. Load curves for compressive and tensile face sheets for Beam 1.

With these materials, a tensile strain of $2.5 \%$ could not be reached unless the beam is more compliant. Compliance can be increased by decreasing flexural stiffness as well as increasing the span. This was taken into account in the following beam design.

\section{Design Iteration 2}

The first and largest change for the Beam 2 design was to decrease the flexural stiffness. This was accomplished by reducing the thickness of the face sheets. The tensile face sheet was reduced from 4 plies to 1 ply and the compressive side was reduced from 8 plies to 2 plies. The face sheets have both been reduced in thickness to a quarter of those in Beam 1. 
This beam was made in the C-channel mold but only used the bottom, flat surface so that there was no curvature on the edges. This resulted in a width of 6.5 inches (17 $\mathrm{cm})$ which was reduced to 5.25 inches $(13 \mathrm{~cm})$ once the edges were cut clean. The crosssection can be seen in Figure 75. The top and bottom face sheets were about 2 and $1 \mathrm{~mm}$ respectively. The core was 1 inch $(25.4 \mathrm{~mm})$ thick.

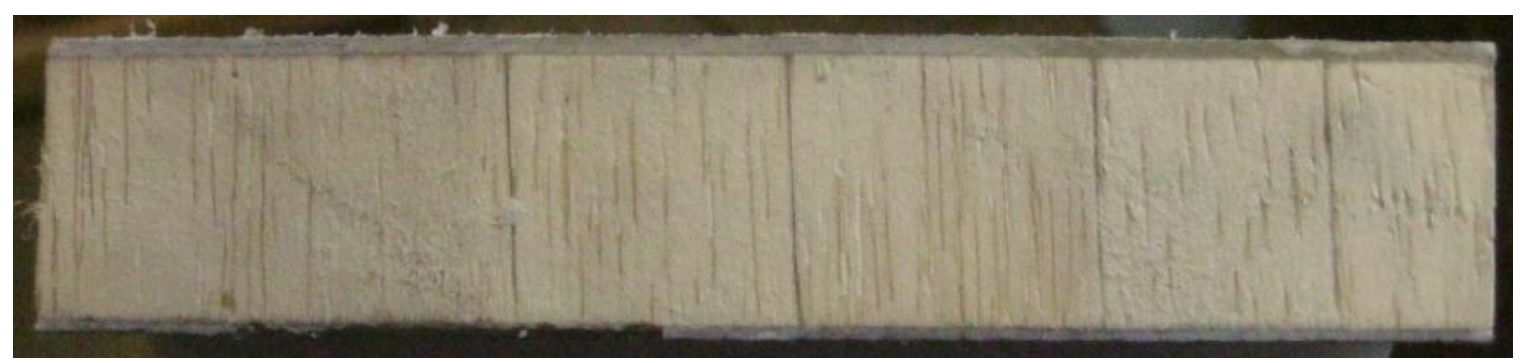

Figure 75: Beam 2 cross-section.

The manufacturing process for this sandwich beam was very different than for the previous beam. Rather than manufacture the face sheets separately and bond them to the core, this beam was shot together as one part. This used the vacuum resin infusion process similar to the previous face sheets and flat plates before, except rather than just lay glass fiber mats, balsa was also placed between the glass mats. This required a second layer of flow media which was placed on the mold side because resin does not pass through balsa as it does through glass mats.

Load pads were made of 3 layers of 0.22 inch $(5.6 \mathrm{~mm})$ thick G10 sheet glued on top of each other. Both their width and length are cut to the width of the beam.

The load rollers were left in the same positions for this test as the previous test because the beams were so different, the failure mechanisms would likely be different and more could be learned at these load roller positions. Therefore, the bottom load nose 
distance was 36 inches $(91 \mathrm{~cm})$ and the top load nose distance was 15 inches $(38 \mathrm{~cm})$. For this thickness of $2.8 \mathrm{~cm}$, the span-to-depth ratio was just under the $36: 1$ minimum recommended.

The failure mechanism was expected to be either balsa shear failure or face sheet failure. Balsa crushing was not expected for this beam because the face sheets were much thinner. Also, the load pads were twice the area which would distribute the load over more area at load application.

\section{$\underline{\text { Beam } 2 \text { Results }}$}

Beam 2 can be seen during testing in Figure 76. The blocks on either side of the beam were not contacting the beam but were a safety measure to keep the beam within the load frame in case of slipping.

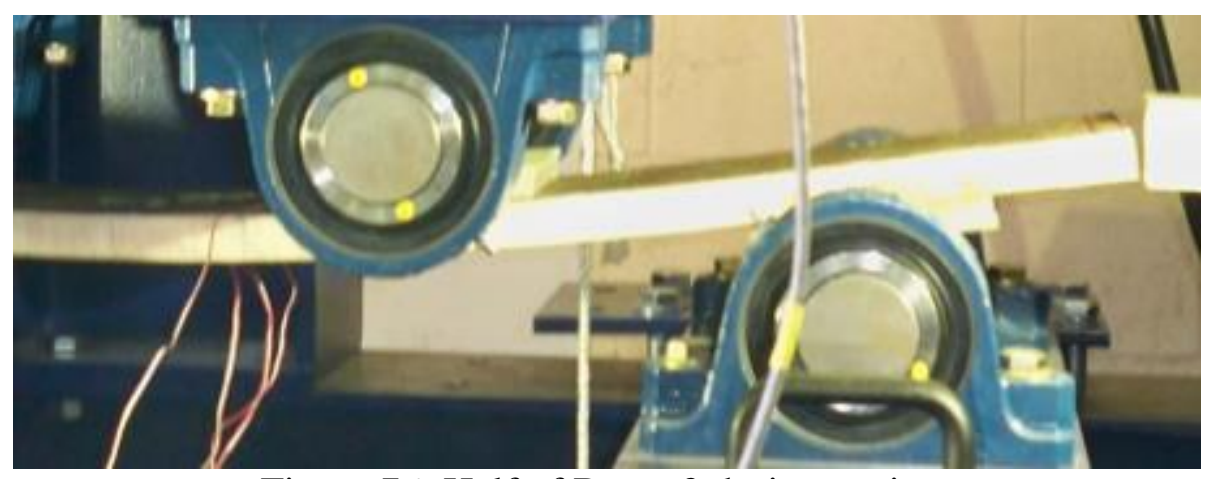

Figure 76: Half of Beam 2 during testing.

This beam reached a maximum load of $12.7 \mathrm{kN}$ at an actuator displacement of 4.3 $\mathrm{cm}$. Strains of $1.26 \%$ and $0.69 \%$ for tension and compression, respectively, were reached as can be seen in the Strain vs. Load plot in Figure 77. Assuming the relative tension to compression strains during the test remain constant, which was seen up to these strain values, then a tensile failure is expected, as discussed here. Tension failures occur at 
$2.5 \%$ strain compared to $1.5 \%$ for compression. This means that the tensile to compressive strain ratio must be greater than 1.7 for a tension failure to occur. The strain ratio here was 1.8 which means that the tensile face sheet would reach its failure strain of $2.5 \%$ before the compressive face sheet reaches its failure strain of $1.5 \%$.

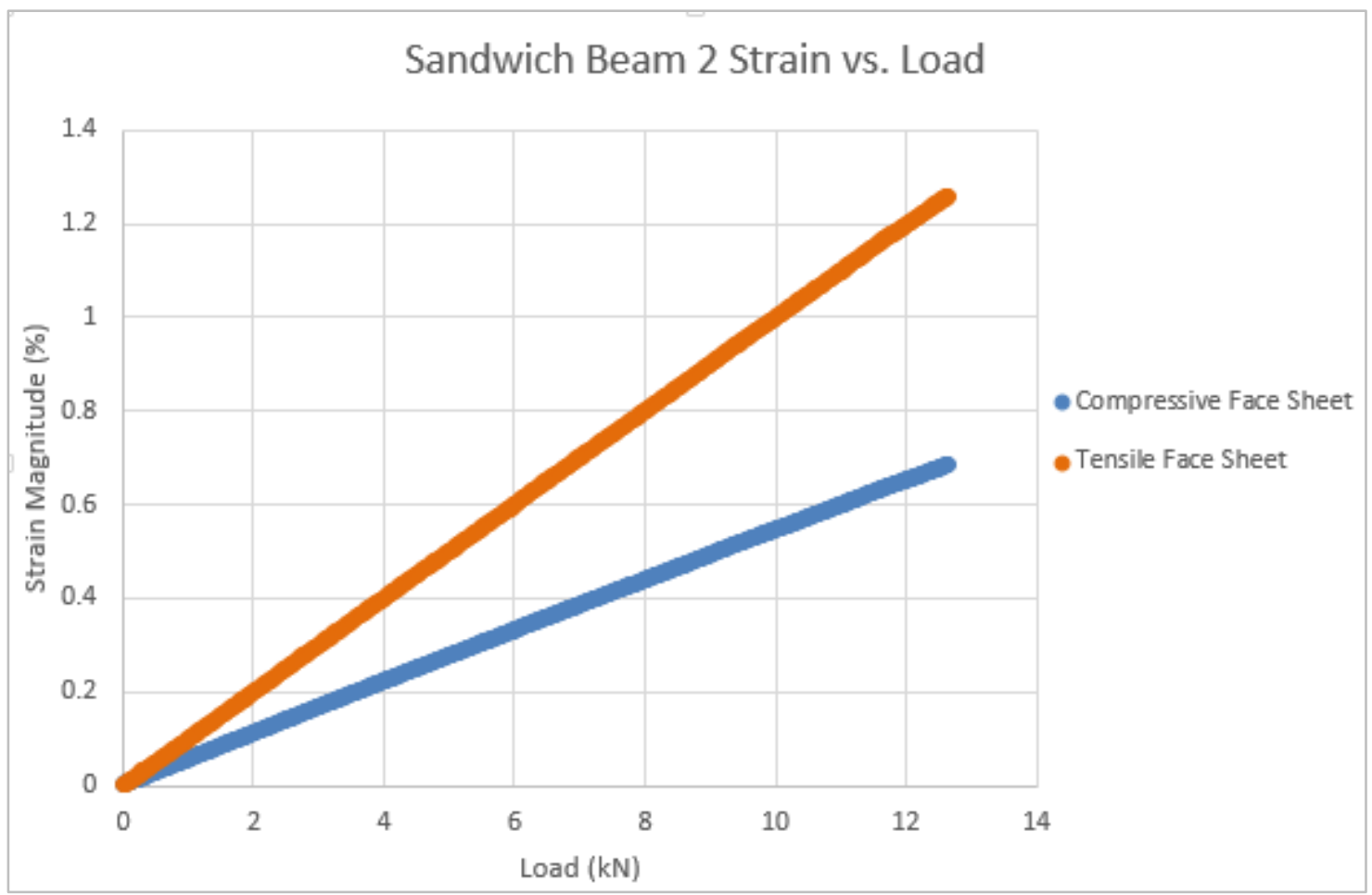

Figure 77: Strain vs. Load curves for compressive and tensile face sheets for Beam 2.

Failure was due to a balsa shear failure which also caused face sheet-core delaminations as seen in Figure 78. The delaminations between face sheets and balsa go from the load pad to sheared balsa on both face sheets. 

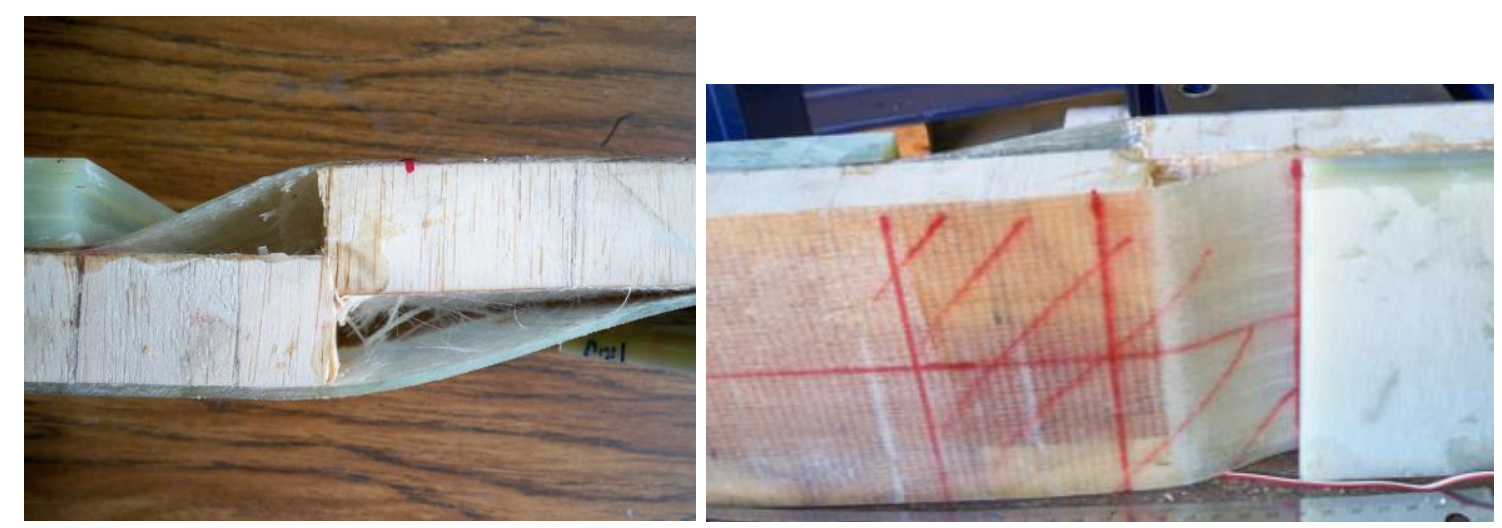

Figure 78: Beam 2 balsa shear failure and delamination.

\section{$\underline{\text { Beam Calculations }}$}

The cross-section used for Beam 2 was kept the same for the next beam. A schematic cross-section in Figure 79 is labeled for calculations. The thickness of the top face sheet is $t_{\mathrm{T}}$, the thickness of the core is $t_{\text {core }}$, and the thickness of the bottom face sheet is $t_{B}$. The beam is loaded so that the top face sheet is in compression and the bottom face sheet is loaded in tension. The total height is $h$, the sum of these thickness. The width is labeled as w. The centroid locations are calculated from the bottom, shown as y.

The calculations are to determine whether a face sheet failure or balsa shear failure will occur and at what load. When face sheet strain in tension or compression exceeds their failure value or maximum shear exceeds balsa shear strength, failure is expected to occur. These are calculated using the beam's flexural modulus, EI. This depends on cross-sectional geometry and elastic moduli of materials used. Determining this value is the first step in determining failure. Calculations do not include the stiffness added by the load pads which is assumed to be negligible. 


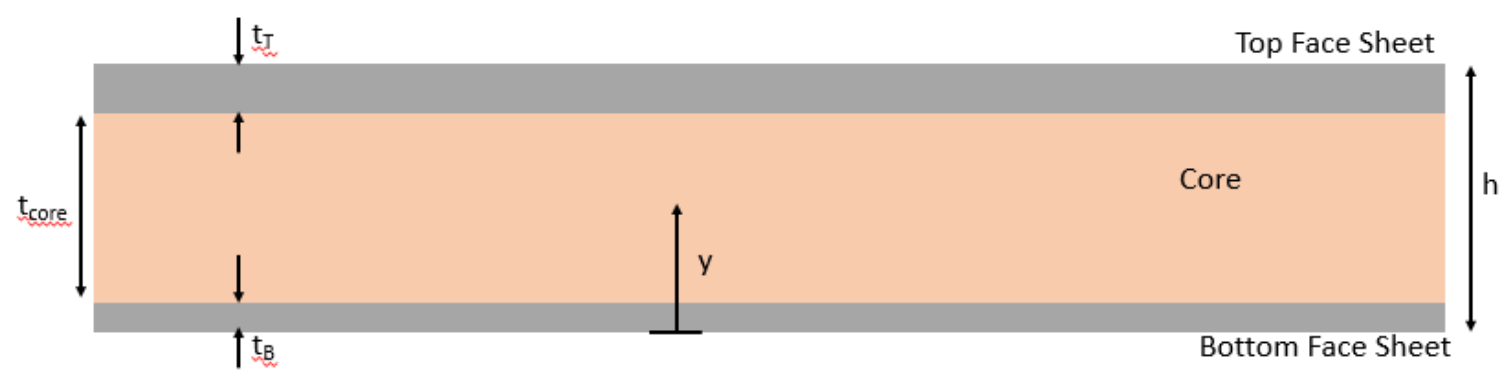

Figure 79: Beam 2 schematic cross-section labeled for calculations.

For these calculations, each face sheet and the core are taken as separate sections.

First, each area is calculated:

$$
\begin{gathered}
A_{T}=t_{T} * w \\
A_{\text {core }}=t_{\text {core }} * w \\
A_{B}=t_{B} * w
\end{gathered}
$$

where variables are labeled in Figure 79 and $A_{T}$ is the area of the top face sheet, $A_{\text {core }}$ is the area of the core, and $A_{B}$ is the area of the bottom face sheet. Next, the centroid location for each section is calculated:

$$
\begin{gathered}
y_{C T}=t_{B}+t_{\text {core }}+\frac{t_{T}}{2} \\
y_{\text {Ccore }}=t_{B}+\frac{t_{\text {core }}}{2} \\
y_{C B}=\frac{t_{B}}{2}
\end{gathered}
$$

where $y_{C T}$ is the location of the top face sheet centroid from the bottom of the beam, $y_{C c o r e}$ is the location of the core centroid, and $y_{C B}$ is the location of the bottom face sheet centroid. Once centroid locations have been determined for each section, the total crosssection centroid location is determined. This requires weighing each section's individual 
centroid location by its elastic modulus. However, due to the face sheet modulus being much larger than that of the balsa core, the core modulus will be approximated as zero to simplify calculations. This results in the elastic moduli not needing to be included because both face sheets have the same elastic modulus.

$$
y_{C t o t}=\frac{y_{C T} * A_{T} * E_{T}+y_{C B} * A_{B} * E_{C}}{A_{T} * E_{T}+A_{B} * E_{C}}=\frac{y_{C T} * A_{T}+y_{C B} * A_{B}}{A_{T}+A_{B}}
$$

Where $y_{\text {Ctot }}$ is the stiffness weighted centroid location of the composite beam crosssection. $E_{T}$ and $E_{C}$ are the elastic moduli for the tensile and compressive face sheets respectively. These are equal and divide out from the equation. Plugging in, the location of the centroid from the bottom is calculated to be $18.4 \mathrm{~mm}$. The next step is calculating each section's area moment of inertia about the beam's composite centroid. This requires using the parallel-axis theorem.

$$
\begin{aligned}
& I_{T}=\frac{w * t_{T}^{3}}{12}+A_{T} *\left(y_{C t o t}-y_{C T}\right)^{2} \\
& I_{B}=\frac{w * t_{B}^{3}}{12}+A_{B} *\left(y_{C t o t}-y_{C B}\right)^{2}
\end{aligned}
$$

Where $I_{T}$ is the area moment of inertia for the top face sheet and $I_{B}$ is for the bottom face sheet. The last step in calculating the flexural modulus is multiplying the elastic modulus for each section by its area moment of inertia about the composite centroid and summing these.

$$
E I=I_{T} * E_{F S}+I_{B} * E_{F S}
$$

Where $E_{F S}$ is the elastic modulus of the face sheets, denoted as $E_{T}$ and $E_{C}$ above. Plugging in elastic moduli and geometric values for Beam 2 from above, the flexural modulus is $2.6 \mathrm{kN}-\mathrm{m}^{2}$. Before continuing, the flexural modulus is calculated for Beam 1 
for comparison. This calculation will not include the curvature or adhesive so that the same general cross-section geometry can be used with different face sheet thicknesses. The flexural modulus for Beam 1 is calculated to be $14.3 \mathrm{kN}-\mathrm{m}^{2}$. This means that Beam 2 is more than 5 times more compliant than Beam 1.

The maximum strain for both face sheets can now be calculated by calculating bending stress for a composite beam and equating to strain with Hooke's Law. Combining these equations results in:

$$
\varepsilon=\frac{M y}{E I}
$$

where $\varepsilon$ is strain, $\mathrm{M}$ is moment, $\mathrm{y}$ is distance from the neutral axis to location of strain calculation, and EI is flexural modulus. The neutral axis is located at the total centroid location calculated above. The moment is calculated with the load roller geometry and load as:

$$
M=a \frac{P}{2}
$$

where $\mathrm{P}$ is the load from the actuator, meaning both top load rollers experience half of this. The moment arm, $a$, is the distance between the bottom load roller and nearer top load roller. Plugging in moment into the strain equation, the strain becomes:

$$
\varepsilon=\frac{a P y}{2 E I}
$$

Maximum compressive strain and maximum tensile strain are at the surfaces. The values for $\mathrm{y}$ for the surfaces are: 


$$
\begin{gathered}
y_{\text {compressive }}=h-y_{\text {Ctot }} \\
y_{\text {tensile }}=y_{C t o t}
\end{gathered}
$$

The accuracy of these calculations is checked by inputting the maximum load reached from the Beam 2 test and comparing strains.

$$
\begin{aligned}
\varepsilon_{\text {compression }}= & \frac{a * y_{\text {topsheet }} * P}{2 E I} \\
& =\frac{(10.5 * 25.4) m m * 10.0 m m *(2866 * 4.44) N}{2 * 2.6 E 9 \mathrm{~N}-m^{2}} \\
& =0.66 \% \\
\varepsilon_{\text {tension }}=\frac{a * y_{\text {botsheet }} * P}{2 E I} & \\
= & \frac{(10.5 * 25.4) m m * 18.4 m m *(2866 * 4.44) N}{2 * 2.6 E 9 \mathrm{~N}-m^{2}} \\
= & 1.21 \%
\end{aligned}
$$

These calculated strains of $0.66 \%$ and $1.21 \%$ in compression and tension are close to the experimental values of $0.69 \%$ and $1.26 \%$, respectively. These equations are rearranged to solve for load at which a face sheet will fail. This is done by inputting $1.5 \%$ failure strain for compression and $2.5 \%$ for tension. The failure loads are $29.3 \mathrm{kN}$ and $26.6 \mathrm{kN}$ for compression and tension respectively. Due to the relative magnitudes, a tension face sheet will occur first. However, this load is 2 times larger than that reached before balsa shear failure in Beam 2. Regardless of load geometry positions, a larger load cannot be reached because balsa shear failure is independent of load roller geometry. Therefore, the moment arm must be increased so that larger strains are reached at lower loads. 


\section{Design Iteration 3}

Based on the previous beam test, it was found that the balsa fails in shear at a load around $12.7 \mathrm{kN}$. This failure load is independent of load roller geometry, only beam material properties and cross-sectional geometry. Therefore, the load rollers must be moved out to increase the distance between the lower and upper load rollers, thus increasing moment in the gage for a given actuator load. Due to limitations on size from the mold, the largest beam possible was made on this mold. This beam was longer but only by 4 inches $(10 \mathrm{~cm})$. This allowed the bottom load rollers to be moved from 36 inches $(91 \mathrm{~cm})$ to 40 inches $(102 \mathrm{~cm})$. The top load roller distance was maintained at 15 inches $(38 \mathrm{~cm})$. This increased the moment arm from 10.5 inches $(27 \mathrm{~cm})$ to 12.5 inches $(32 \mathrm{~cm})$.

The load pads used for this beam were only 1 layer of the G10 material whereas the previous beam used 3 layers. This was to reduce the stiffness discontinuity near the load pads which was expected to increase the load at which balsa shear failure occurred.

This sandwich beam was instrumented with only 2 strain gages, one in the middle of the gage section of each face sheet. Looking at data from the previous sandwich beam strain gages, torsion was not an issue. This was assumed to be the case for this beam and following beams which means that the strain gage array was not necessary.

\section{$\underline{\text { Beam } 3 \text { Results }}$}

This beam reached a load of $12.2 \mathrm{kN}$ and an actuator displacement of $6.1 \mathrm{~cm}$ before failure due to balsa shear failure. This resulted in maximum tensile and compressive strains of $1.32 \%$ and $0.65 \%$, respectively. Comparing to Beam 2, Beam 3 
failed at a slightly lower load but higher strains. Balsa shear failure depends on the load, not the load geometry, so a similar failure load was expected and achieved. Also as expected, this beam reached a higher displacement at a lower load due to the larger span. The Strain vs. Load curves for both face sheets can be seen in Figure 80 .

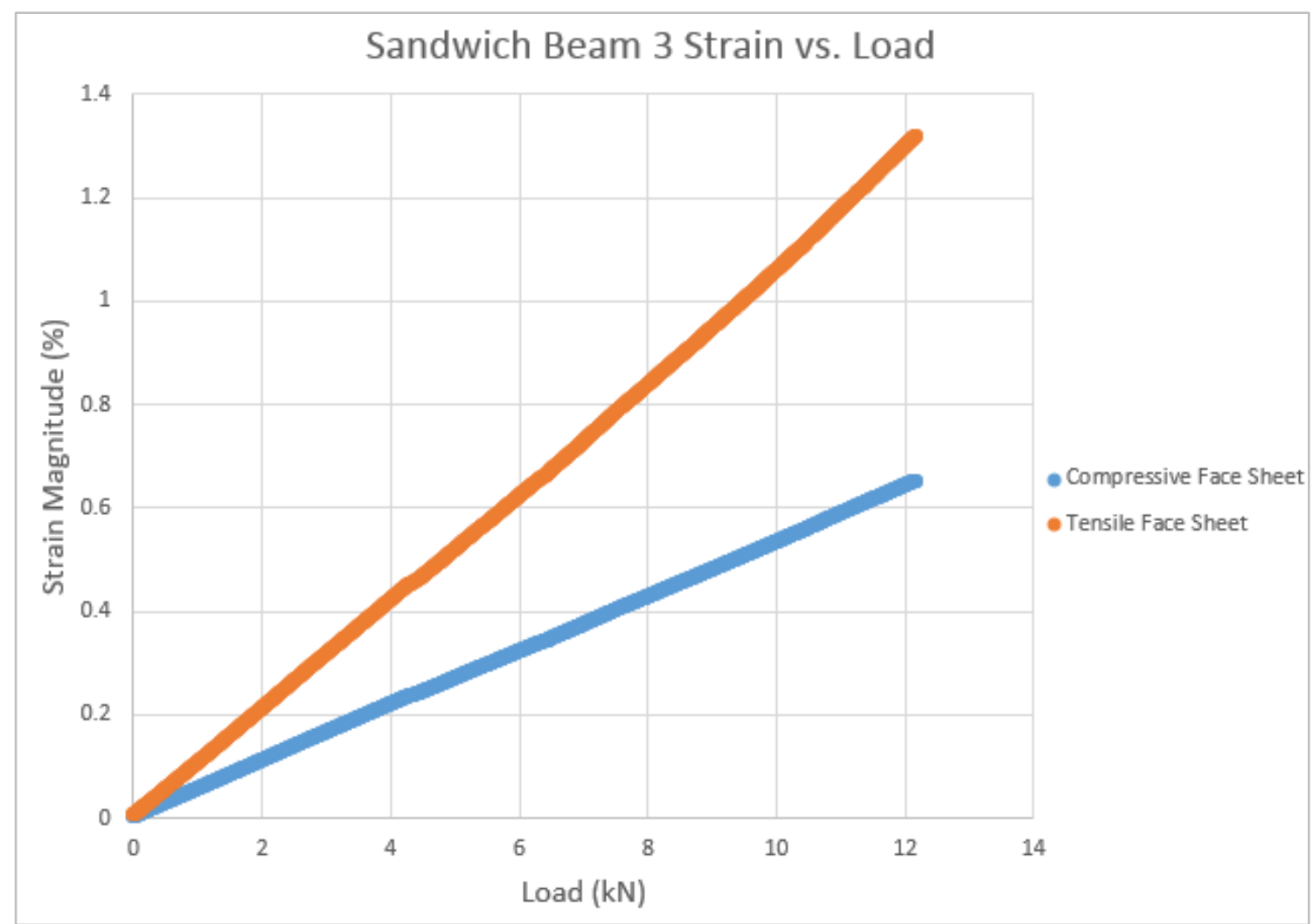

Figure 80: Strain vs. Load curves for compressive and tensile face sheets for Beam 3.

Plugging this load roller geometry and failure load into Equation 20 above, the tensile and compressive strains at this load were calculated to be $1.39 \%$ and $0.75 \%$ respectively. These are close to the experimental strains achieved. 


\section{Design Iteration 4}

The load roller distances must increase in order to get failure strains before the balsa fails in shear. This was accomplished by implementing a new mold to make larger beams. This allowed for the lower load roller distance to reach 88 inches $(224 \mathrm{~cm})$, the maximum of the frame. The top load rollers were moved to 30 inches $(76 \mathrm{~cm})$ to keep an approximate thirds ratio roller distance. The span-to-depth ratio for this beam was just under 88:1 which would give an upper limit.

This beam was made similarly to the previous beams except that it was made on a new mold. The maximum length of the old mold was already met so a $10 \mathrm{ft}$ aluminum mold was made. This was a quarter inch $(0.6 \mathrm{~cm})$ thick aluminum plate, $10 \mathrm{ft}(3 \mathrm{~m})$ long and $1 \mathrm{ft}(30 \mathrm{~cm})$ wide. The previous beams were post-cured in the oven however this mold was too large to fit. Therefore, heating pads were used for the curing process. Heating pads are adhered to the bottom of the mold. A wood frame was made to elevate the mold 3.5 inches $(9 \mathrm{~cm})$ above the table, only touching the frame along the edges. This left room for fiberglass insulation on the underside. During the curing process, fiberglass was also placed on top of the beam. As for previous beams, injection of on this mold injected resin at one end and pulled vacuum at the other. The mold ready for injection can be seen in Figure 81. 


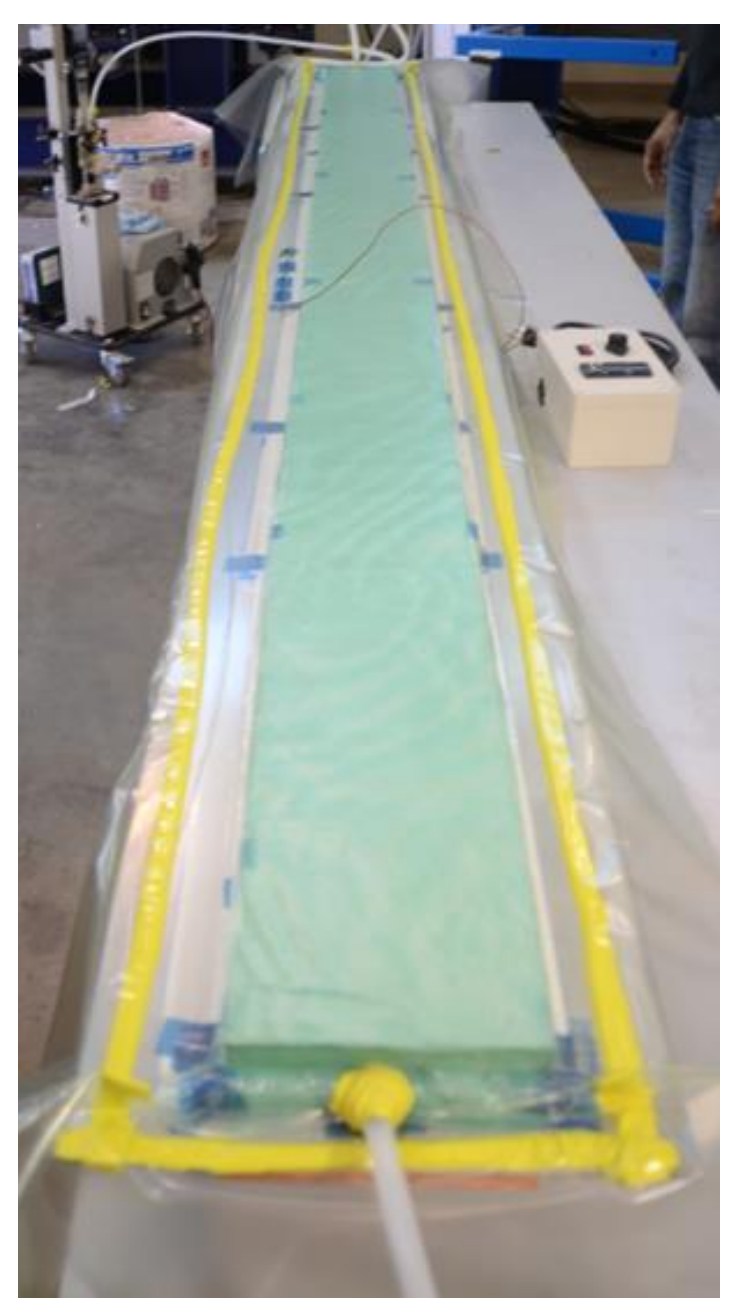

Figure 81: Beam 4 on mold and under vacuum ready for injection. Injection on close end and vacuum on far end.

The temperature of the heating pads was controlled with a feedback loop. This required a thermocouple feeding into a controller which is shown in Figure 82. This thermocouple was placed halfway down the length of the mold. The controller was set to keep the temperature $70 \mathrm{C}$ for 12 hours. 


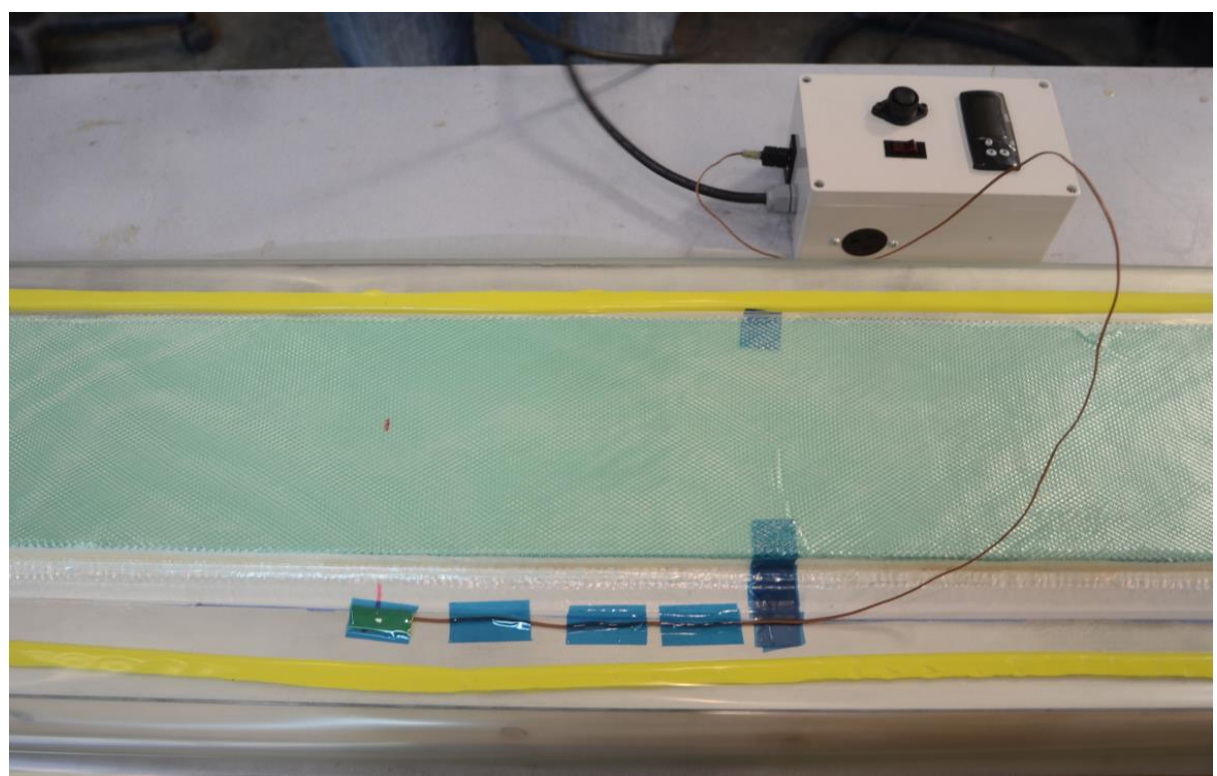

Figure 82: Thermocouple and controller on mold just before injection.

It was desired to measure the temperatures of each face sheet during the curing process to check that the proper temperature was reached and was held for the proper amount of time, without significant overshoot. It was also important that the temperature of each face sheet be similar to avoid uneven curing and warping. Thermocouples were used to record these temperatures with one on the top face sheet and the other on the mold near the bottom face sheet. It was assumed that the temperature of the mold was similar to the bottom face sheet and this also allowed the accuracy of the controller to be investigated. All three thermocouples were under tacky tape and the locations can be seen in Figure 83. The data acquisition for the log thermocouples is the Omega HH306A Data Logger. 


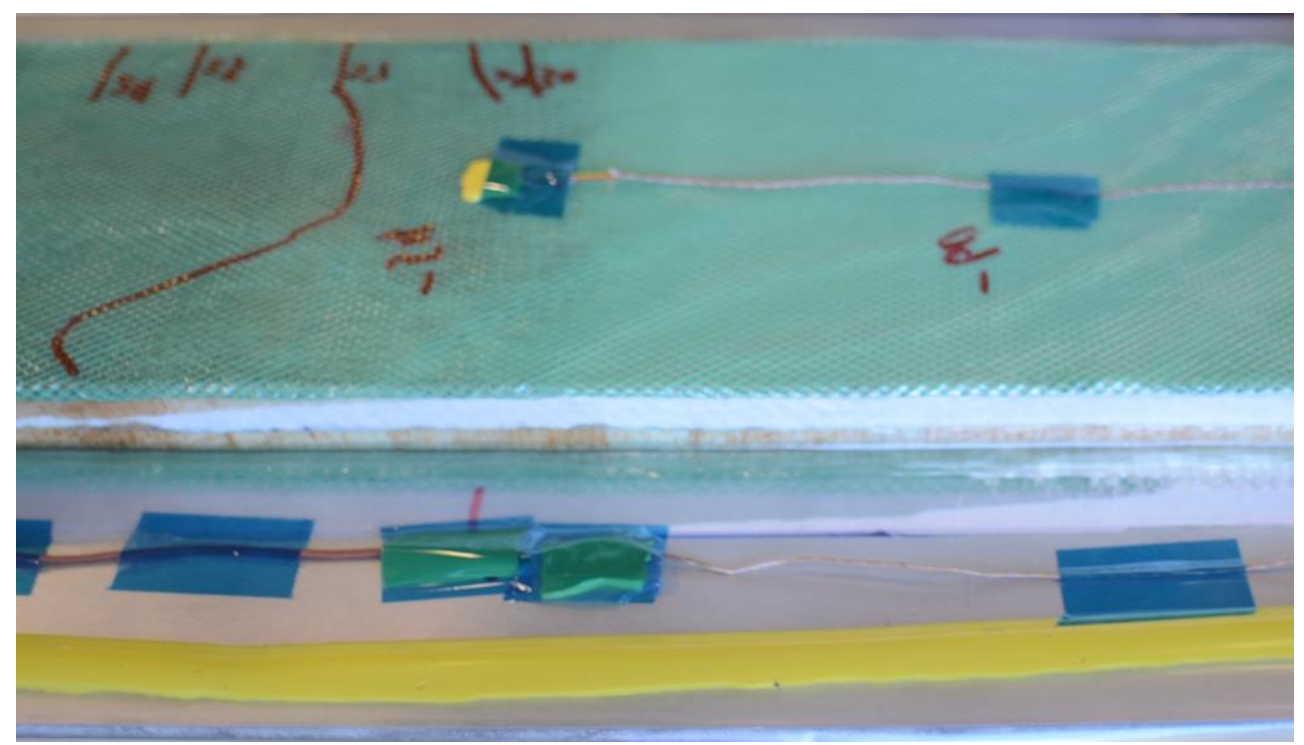

Figure 83: All three thermocouples on beam and mold during injection. 1 controller thermocouple on mold and 2 logging thermocouples.

After cutting the edges clean, this beam was 108 inches $(274 \mathrm{~cm})$ long and 5.75

inches $(15 \mathrm{~cm})$ wide. Load pads were made two layers of 5/16 inch $(0.8 \mathrm{~cm}) \mathrm{G} 10$ material. These were cut to width of the beam and 7 inches $(18 \mathrm{~cm})$ long. They were longer than previous load pads because more deflection was expected which would cause more displacement of the load pads to rolling on the load rollers. This sandwich beam was instrumented with 2 strain gages, one in the center of each face sheet.

\section{$\underline{\text { Beam } 4 \text { Results }}$}

This beam can be seen in the test frame in Figure 84. During testing, this beam was displaced by about $11.7 \mathrm{~cm}$ before maximum actuator travel was reached. No failure was reached by this displacement. However, this is not the total travel possible for the actuator. The actuator displacement is set at zero when the top and bottom load rollers are separated by about 7.75 inches $(20 \mathrm{~cm})$. Therefore, given the thickness of this beam with load pads, only about $11.7 \mathrm{~cm}$ were available for travel. Reaching this displacement 
required a load of $2.1 \mathrm{kN}$ and resulted in maximum tensile and compressive strains of $0.51 \%$ and $0.30 \%$ respectively. The beam at this $11.7 \mathrm{~cm}$ displacement can be seen in Figure 85. The Strain vs. Load curves for both face sheets can be seen in Figure 86.

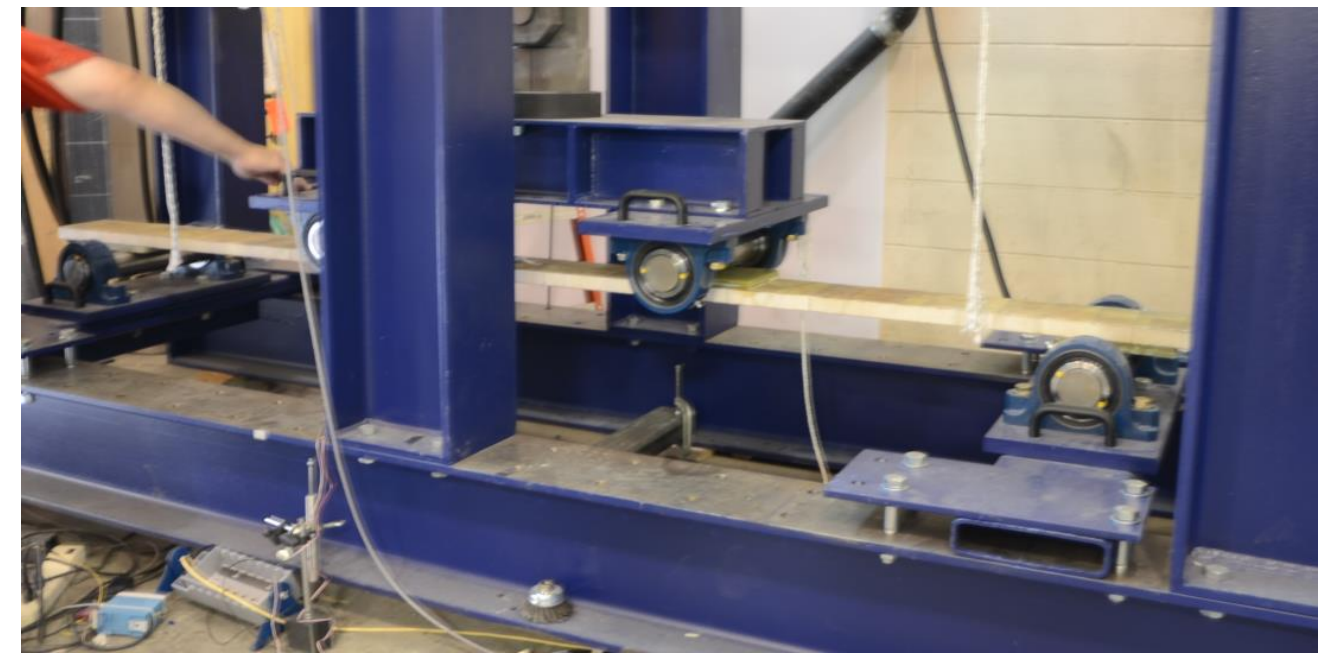

Figure 84: Beam 4 in test frame before applying load.

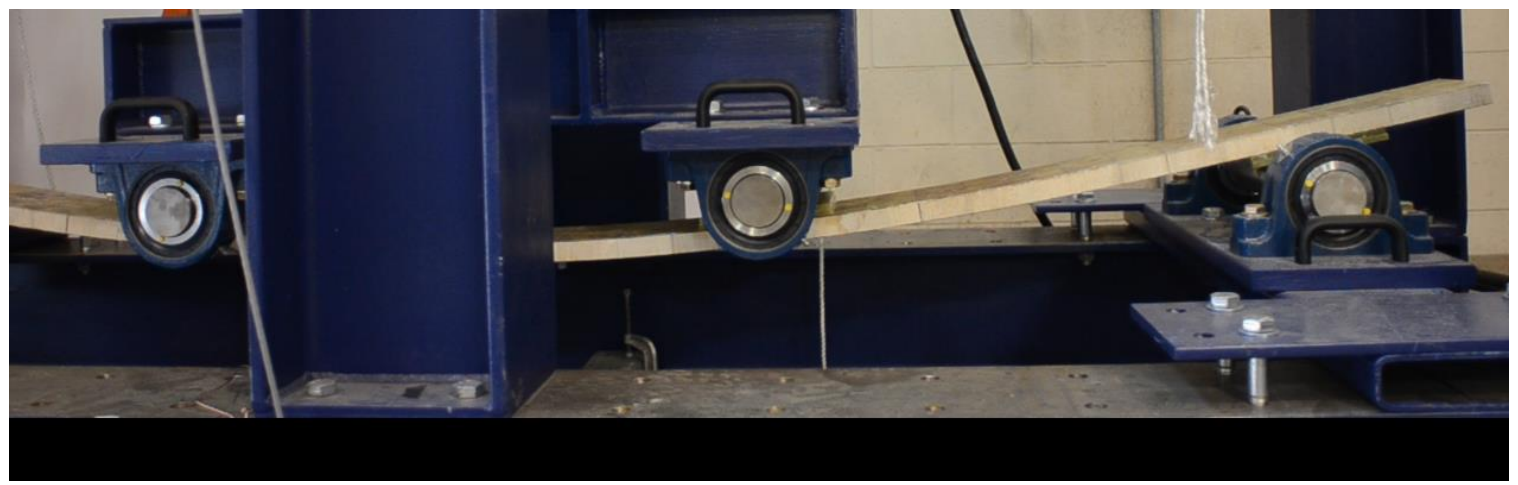

Figure 85: Beam 4 loaded and at maximum actuator displacement before adjusting to take advantage of full actuator travel. 


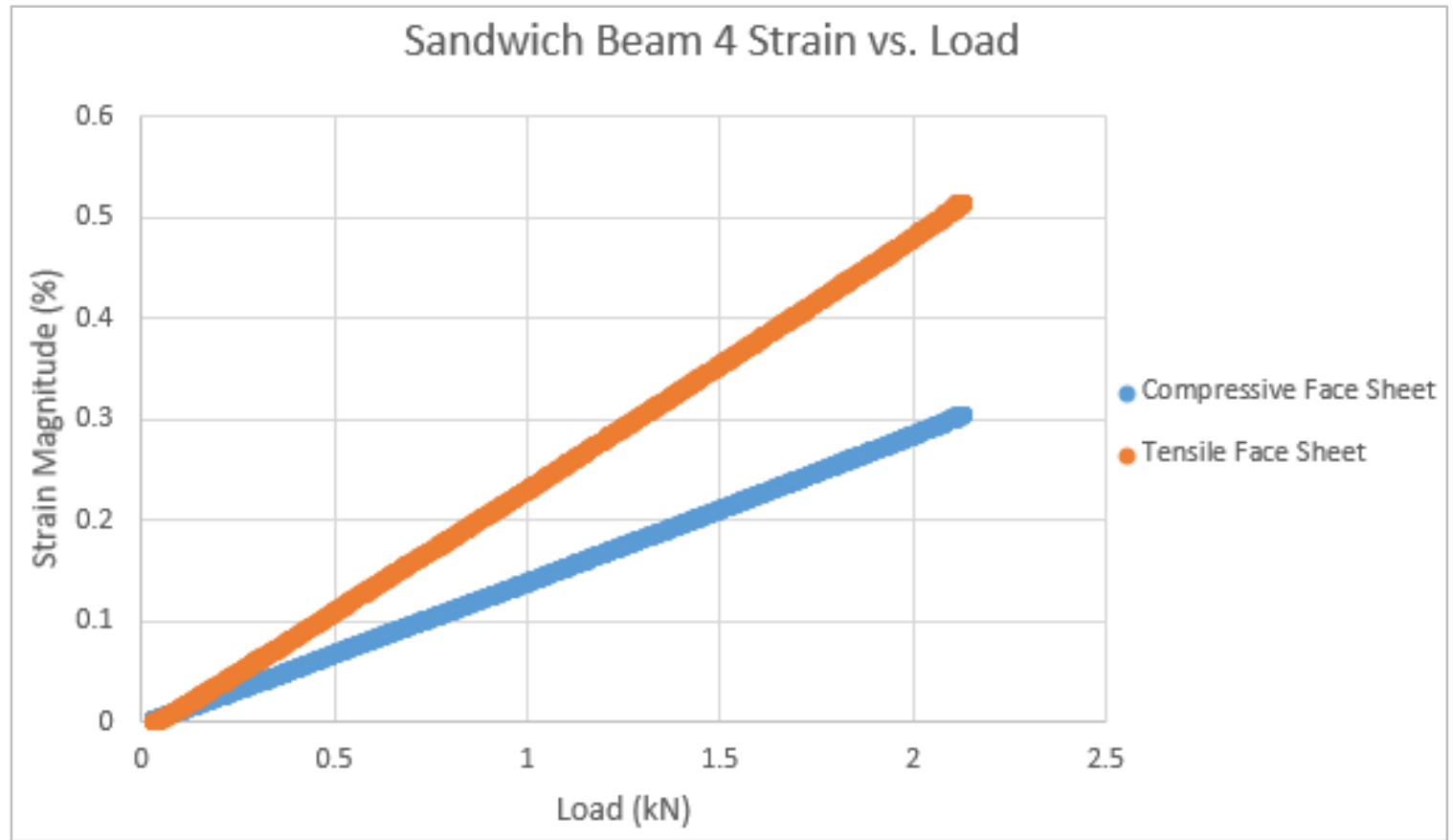

Figure 86: Strain vs. Load curves for compressive and tensile face sheets for Beam 4.

Based on Equation 20 above, the tensile and compressive strains at this load for this load roller geometry are $0.56 \%$ and $0.30 \%$ respectively. These calculated strains are close to experimental even for this longer beam with higher deflection.

The beam was retested with large wooden spacers so that the entire actuator travel could be used. The beam during testing near the maximum displacement reached with the wood spacers is shown in Figure 87. Data was not recorded for this, but even with a displacement just under the maximum actuator travel, the strain was just above $1 \%$. This load roller geometry results in the beam being too compliant whereas previous tests were too stiff. 


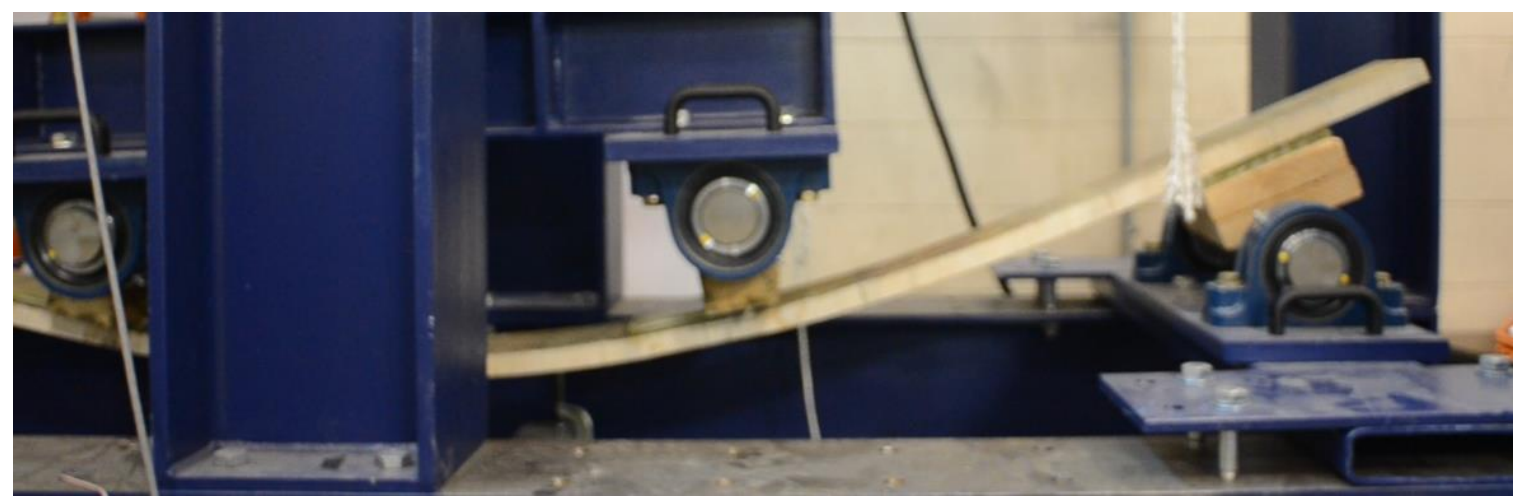

Figure 87: Beam 4 loaded and near maximum actuator travel reached by using wooden spacers shown.

Plugging into Equation 20 above and calculating for failure load, it is expected that a tensile face sheet failure would occur at $9.8 \mathrm{kN}$ which is below the balsa shear failure. The problem is that this will require a displacement outside the range of the actuator. Due to difficulties with reaching a tensile failure strain of $2.5 \%$ within the actuator travel and below the balsa shear failure load, compressive failure is attempted. This would require a strain of $1.5 \%$ for failure.

\section{Design Iteration 5}

A compressive face sheet failure is expected to be easier than tensile because it needs to reach a lower strain for failure. For consistency, the same cross-sectional area was used for this beam as the three previous beams. The difference was that this beam was loaded with 1 ply on top and 2 plies on bottom in order to force a compressive failure to occur before tensile. Due to the flexural stiffness being dependent on cross-sectional geometry and material properties only, this beam had the same flexural stiffness as previous beams. Therefore, in order to make a failure occur at a lower displacement as desired, a shorter span was used. Load roller locations in which failure would be most 
likely was implemented which included a span of 62 inches $(158 \mathrm{~cm})$ with a top load roller distance of 20 inches $(51 \mathrm{~cm})$. This results in a span-to-depth ratio very close to the 60:1 recommended. Plugging into Equation 20 above with this geometry, failure loads for compressive and tensile face sheets were $8.0 \mathrm{kN}$ and $24.4 \mathrm{kN}$, respectively. This means that a compressive failure was expected to occur before a tensile failure and also before a balsa shear failure. Due to difficulties with injecting down the length of such a long beam, this beam was injected from the middle with vacuum ports on either end. There was an injection port on either side which went into spiral tubing, each of which extended two-thirds the length of the beam. The vacuum ports both had spiral tubing across the width of the beam at either end. This can be seen in Figure 88.

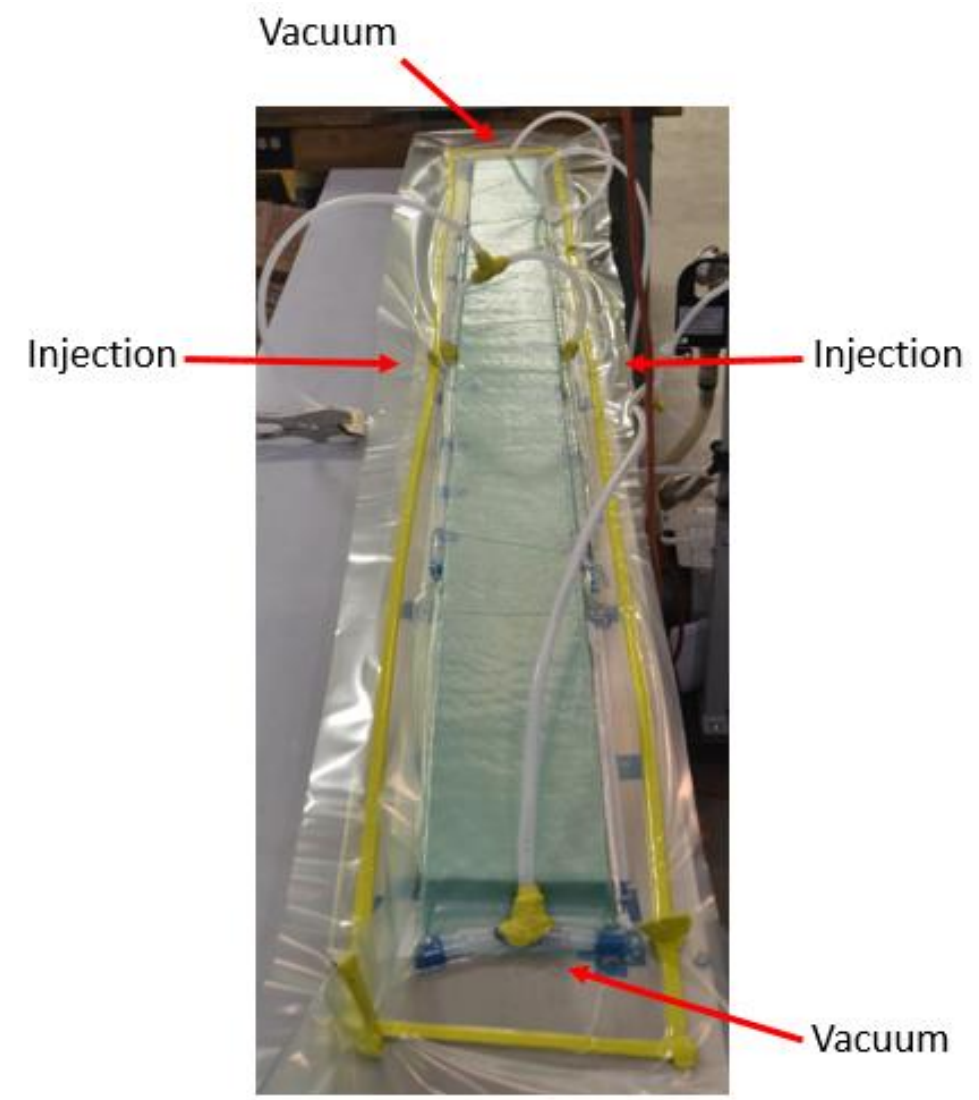

Figure 88: Beam 5 with middle injection and end vacuum ports. 


\section{Beam 5 Results}

This beam experienced a compressive face sheet failure as expected. This occurred at load of $6.2 \mathrm{kN}$ and an actuator displacement of $8.1 \mathrm{in}$. The compressive and tensile face sheet strains at failure were $1.08 \%$ and $0.61 \%$ respectively. This failure occurred at a lower strain and load than expected. This was likely due to balsa compressive failure causing premature face sheet failure as discussed below. The failure can be seen in Figure 89. The Strain vs. Load curves for both face sheets can be seen in Figure 90. The curve starts at $1.4 \mathrm{kN}$ because the large spacers used caused a slight preload in the beam of $1.4 \mathrm{kN}$. The kink around $3.7 \mathrm{kN}$ was due to hammering a shifting spacer back into place.

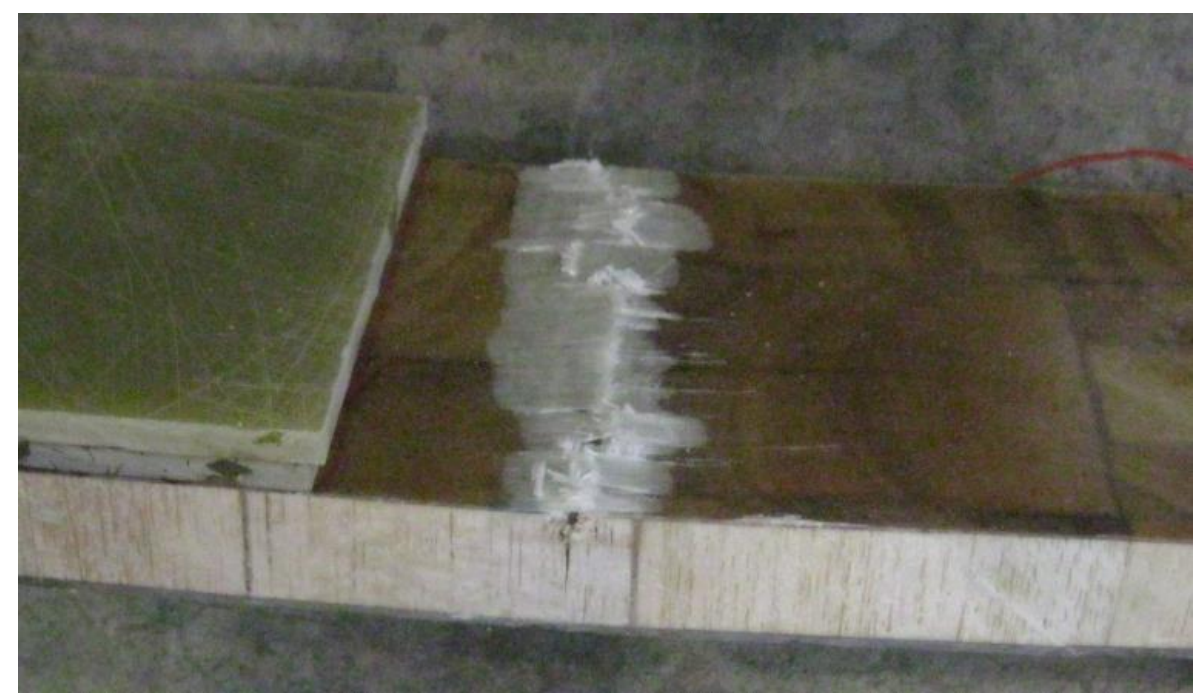

Figure 89: Beam 5 compressive face sheet failure. 


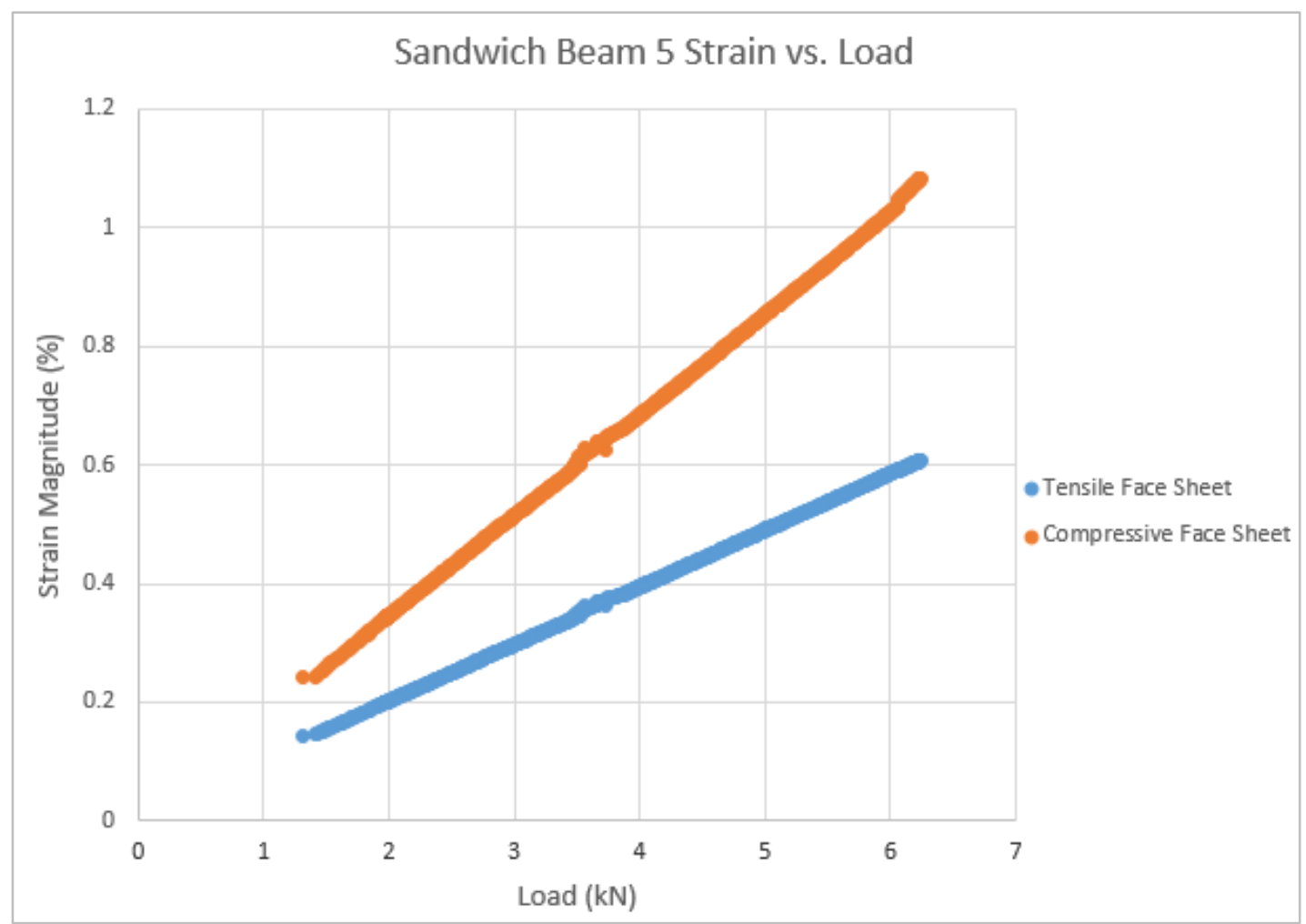

Figure 90: Strain vs. Load curves for compressive and tensile face sheets for Beam 5.

\section{$\underline{\text { Design Iteration } 6}$}

Beam 6 was the same as Beam 5 except that a wave was now added to the 1 ply, compressive face sheet. This was a discontinuous fiber in-plane (DFIP) wave which extended across the whole width. The DFIP wave geometry was the same as those studied previously, with a misalignment angle around 29 degrees. This wave type was chosen for a number of reasons. A wave that has previously been studied was desired so that direct comparisons could be made. The DFIP wave was chosen over CFIP because this flaw results in a lower strain at failure making a face sheet failure more likely. Lastly, as mentioned above, the CFIP wave may require more parameters to be fully characterized. Therefore, comparisons could not be made to the CFIP wave coupons 
without an extensive study which is recommended as future work. Therefore, this beam was made same as before except utilizing the method for forming DFIP waves as outlined by Nelson, 2013. The wave in the 6.5 inch $(17 \mathrm{~cm})$ wide glass mat to be the compressive face sheet can be seen in Figure 91. This beam had one strain gage in the middle of the gage section on the tension side face sheet. Four strain gages were used on the compressive side to investigate any possible load redistribution due to the wave. This can be seen in Figure 92 below.

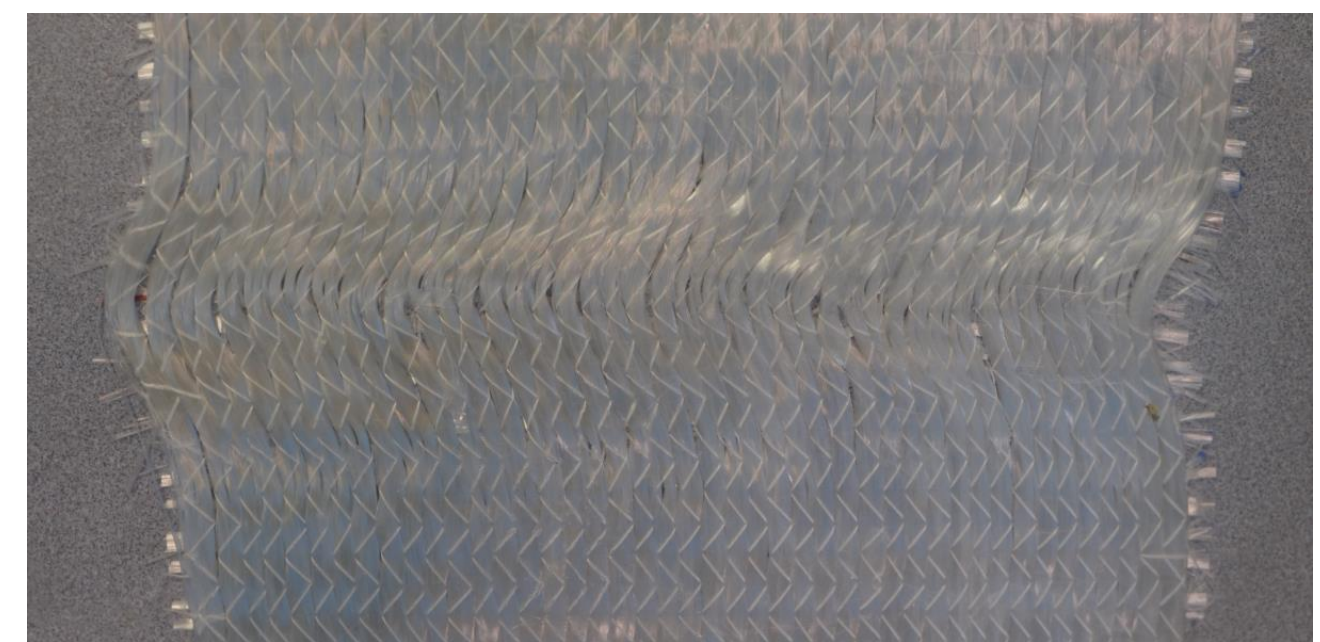

Figure 91: 6.5 inch $(17 \mathrm{~cm})$ wide glass mat to be compressive side face sheet showing DFIP wave.

\section{$\underline{\text { Beam } 6 \text { Results }}$}

This beam failed in the wave in compression as shown in Figure 92. The crack propagated across the width through the maximum misalignment angle. This beam reached a load of $4.1 \mathrm{kN}$ and an actuator displacement of only $5.1 \mathrm{~cm}$. The compressive strains at failure were around $0.69 \%$ and the tensile strain was $0.36 \%$. The Strain vs. Load curves for all 5 strain gages are included in Figure 93. Again, the load starts at 1.4 $\mathrm{kN}$ due to the preload caused by the spacers. The strain for Compressive Face Sheet 4 
starts at a lower compressive preloaded value and is much less stiff over the course of the test. This is most likely due to a damage to the sensor caused by the preloading process and/or poor bonding to the face sheet. It appears that any torsion or uneven strain distribution caused by the wave are negligible.

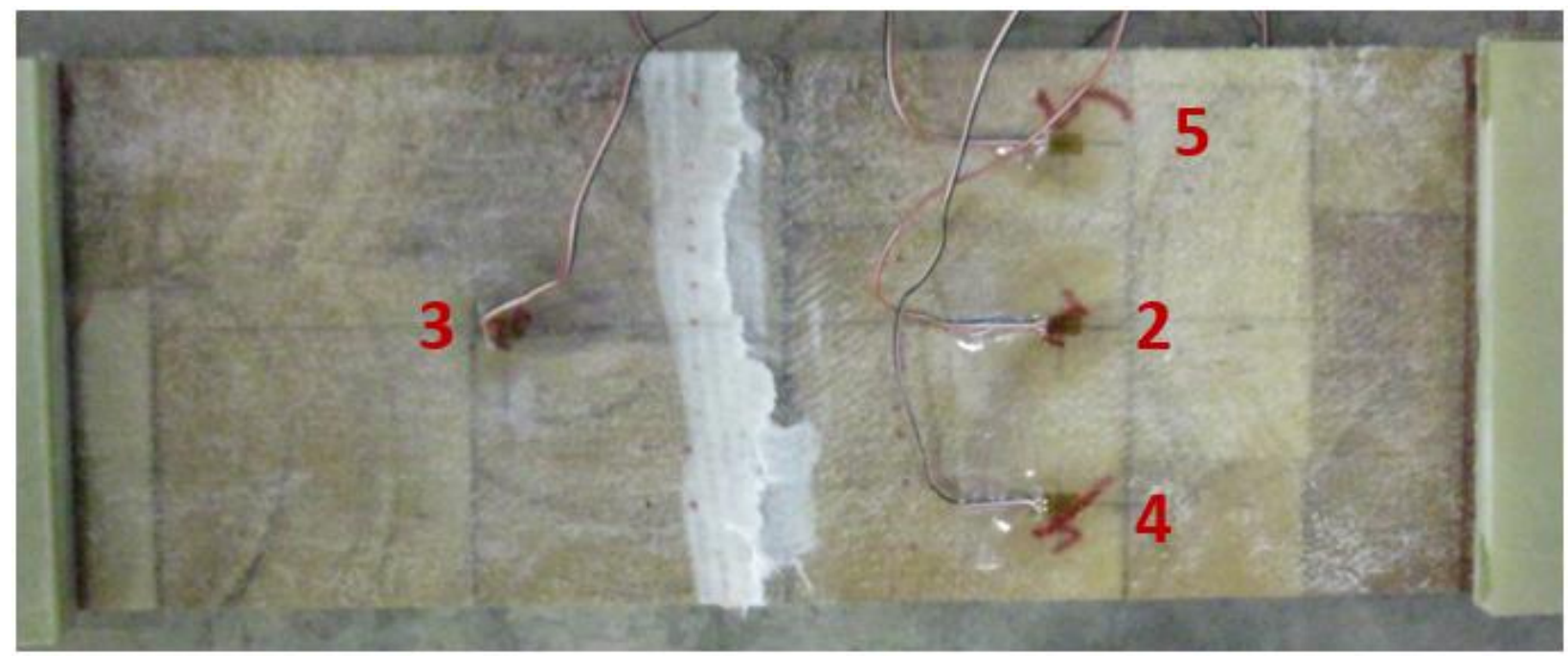

Figure 92: Beam 6 compressive face sheet with strain gage array and failure. Wave extends across the width with maximum amplitude along the middle. Failure occurs near maximum misalignment angle and extends across the width. 


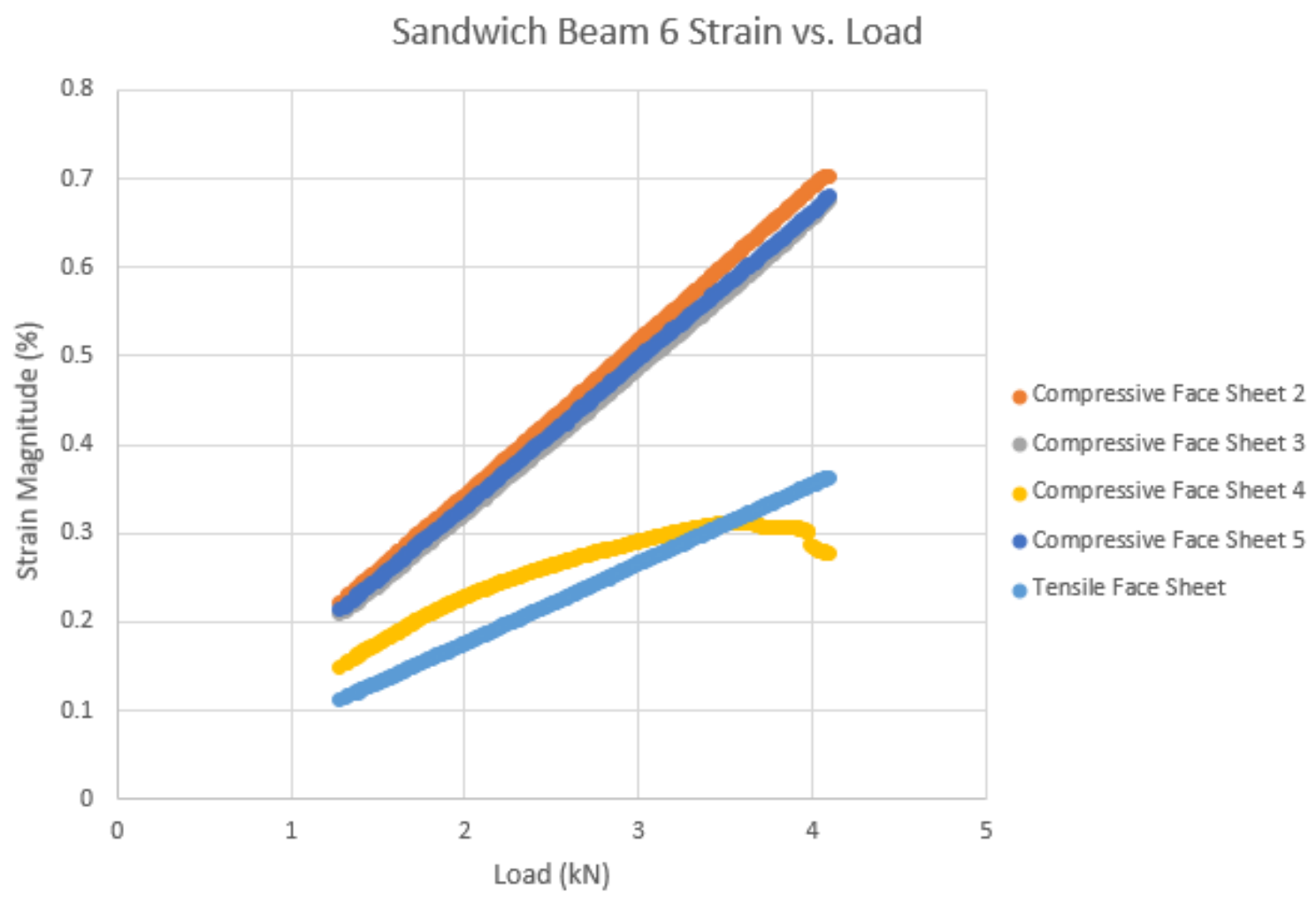

Figure 93: Strain vs. Load curves for compressive and tensile face sheets for Beam 6. Compressive face sheet strain gage locations as shown in Figure 92.

\section{Compressive Flawed Face Sheet Investigation}

Along with developing the sandwich beam test specimen, a small investigation of flawed compressive face sheets is performed. Two more beams of the same design as Beam 6 have been made and tested to increase statistical significance for comparisons to the coupon level. The compressive face sheet strain at failure is compared between flawed beams. These values are then compared to previous coupon tensile data with the same size DFIP wave.

Figure 94 is Stress vs. Strain Magnitude for the compressive face sheets for each beam. Stress is plotted here rather than load to account for the slight differences in width 
of these three beams, allowing for direct comparisons. The data for Compressive Face Sheet 5 for Beam 6 in Figure 93 was chosen as representative for Beam 6 because it is the average of the three non-damaged strain gage readings. It can be seen that the curves are very similar in stiffness but have varying ultimate stresses.

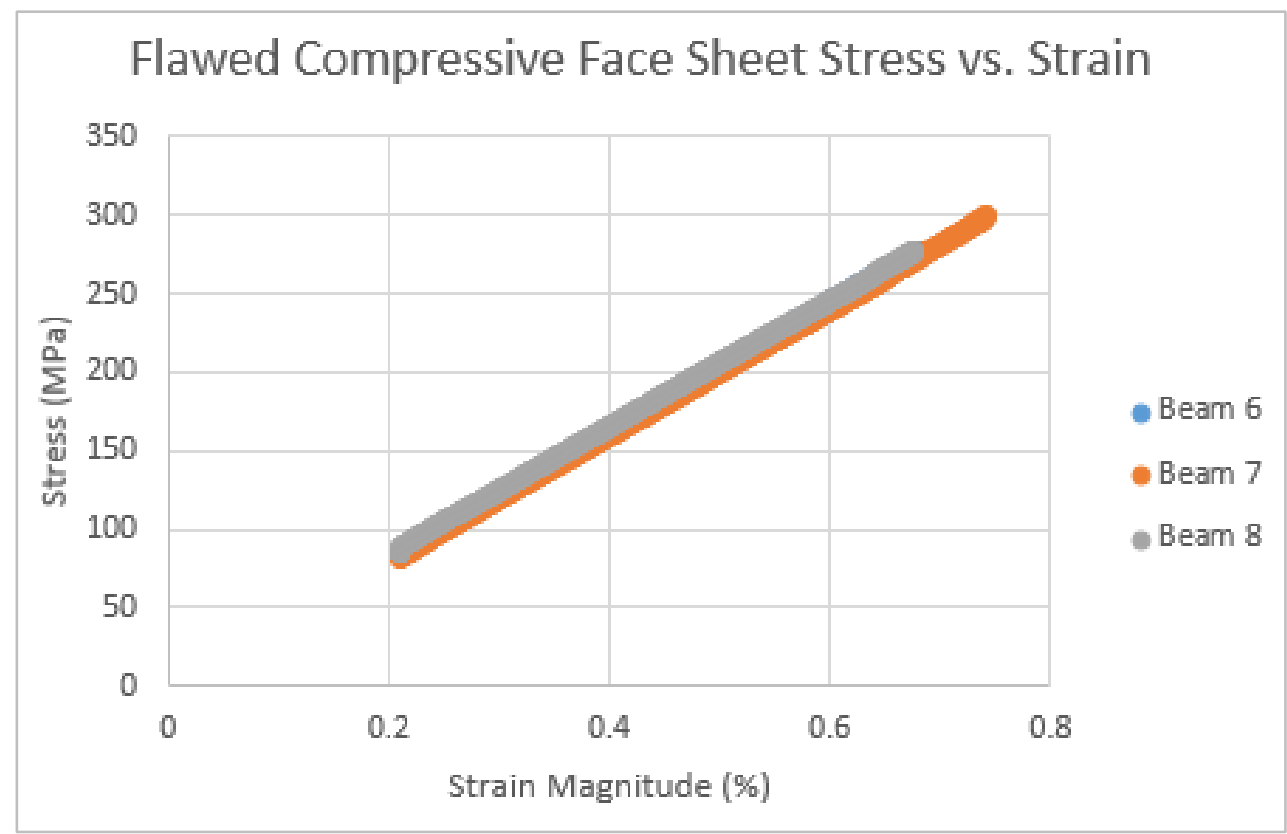

Figure 94: Stress vs. Strain Magnitude for far-field, flawed compressive face sheets for sandwich beams 6 through 8 .

The Beam 6 curve in Figure 94 cannot be seen because it lies directly behind the Beam 8 curve. The stresses here are calculated with Equation 17 above and Hooke's Law. Ultimate stresses as well as strains at failure for each beam can be seen in Table 9 . 
Table 9: Sandwich beam ultimate stress and strain at failure values.

\begin{tabular}{|r|r|r|}
\hline $\begin{array}{c}\text { Sandwich } \\
\text { Beam \# }\end{array}$ & $\begin{array}{c}\text { Ultimate Stress } \\
\text { (MPa) }\end{array}$ & $\begin{array}{c}\text { Compressive Strain } \\
\text { at Failure (\%) }\end{array}$ \\
\hline 6 & 277 & 0.68 \\
\hline 7 & 300 & 0.74 \\
\hline 8 & 259 & 0.68 \\
\hline Average & 279 & 0.70 \\
\hline
\end{tabular}

The average compressive ultimate stress for the sandwich beam face sheets was $279 \mathrm{MPa}$ and the average strain at failure was $0.70 \%$. These can be compared to the coupon data which had an ultimate stress of $257 \mathrm{MPa}$ and a strain at failure of $0.84 \%$ (Nelson, 2013). These values are organized in Table 10.

Table 10: Comparison of average compressive ultimate stress and strain at failure values for sandwich beams and coupons, both with similar DFIP wave.

\begin{tabular}{|c|c|c|}
\hline & $\begin{array}{c}\text { Ultimate Stress } \\
\text { (MPa) }\end{array}$ & $\begin{array}{c}\text { Strain at } \\
\text { Failure (\%) }\end{array}$ \\
\hline $\begin{array}{c}\text { Sandwich Beam } \\
\text { Coupon }\end{array}$ & 279 & 0.70 \\
\hline
\end{tabular}

Comparing the sandwich beam and coupon values it can be seen that the sandwich beam face sheet reaches a higher ultimate stress but a lower strain at failure. This means that the face sheet, when stabilized by the core, is stiffer than the coupon. The variation in strain may be due to measuring methods. The coupon strain was measured over the entire $100 \mathrm{~mm}$ coupon length using the DIC. The DIC could not be used on the beam due to space limitations, therefore, the beam strain was measured outside of the wave region using a strain gage. The strain outside the wave is expected to 
be smaller than in the wave so the difference in methods would result in smaller strain readings in the beams than in the coupons.

\section{$\underline{\text { Sandwich Beam Discussion and Summary }}$}

The goal of this sandwich beam research was to prepare the sandwich beam test specimens. This would enable future work to move up in test scale and bridge the gap between the extensive coupon level testing and full-scale wind turbine. Moving up to a sandwich beam in flexure would also give more representative compression data than the coupon level. The 4 types of test specimen failures desired were both tension and compression face sheet failures both with and without an IP wave. The unflawed failures were desired to investigate the effect of the core material and develop a baseline control on the sandwich beam level. The flawed failures were desired to investigate the effects of defects in flexure. This research would allow for wind turbine blade safety factors to be less overdesigned than those based on coupon level testing.

While the main goal of the sandwich beam research herein was to develop the sandwich beam test specimen, a brief study of the effects of defects has also been performed. This study investigated 3 similar sandwich beams with an IP wave in the compression side face sheet, allowing comparison between the effect of this flaw in both the coupon and structure scales.

\section{Control Beams}

Sandwich Beams 1 through 4 were attempts at creating a control sandwich beam which would fail in the tension side face sheet. These beams were tested between 36 
inch $(91 \mathrm{~cm})$ and 88 inch $(224 \mathrm{~cm})$ spans, keeping a roughly one third ratio for top load roller locations. Through testing and calculations it has been determined that, with the given materials, an unflawed tensile face sheet failure cannot be achieved. At short spans the balsa fails in shear and at long spans the actuator travel is not large enough. The largest tensile strain reached was $1.39 \%$ and failure is expected around $2.5 \%$.

Beam 5 was an attempt at creating a control sandwich beam which would fail in the compression side face sheet. This beam was tested at a span of 62 inches $(158 \mathrm{~cm})$. This beam reached a compressive failure strain of $1.08 \%$ when expected strain at failure is $1.76 \%$ based on BMT coupon tests. Strain at failure is expected to be higher in the sandwich beam than coupons but a lower strain was achieved. This was likely due to balsa compression failure.

The balsa core is required to carry more load nearer to the edge by the single ply face sheet rather than the double ply face sheet. Therefore, if the balsa is to fail in tension or compression, it would be near the single ply face sheet. This did not occur for beams loaded with the single ply face sheet in tension because balsa is stronger in tension. If the balsa failed in compression then the face sheet would be required to hold more load and would locally lose stability added by the balsa which could cause localized failure. As can be seen in Figure 89, failure in the balsa can be seen near the face sheet failure. Therefore, this was not purely a face sheet failure but likely a core failure resulting in face sheet failure.

A tensile face sheet failure was not attempted at the 62 inch $(158 \mathrm{~cm}) \mathrm{span}$ because tensile strain would be the same as compressive strain for Beam 5. Beam 5 
reached a strain of $1.08 \%$ at a load about half that resulting in balsa shear. Assuming linearity, as has been seen thus far, this would result in a tensile strain of $2.16 \%$ before balsa shear failure. This is still below the $2.5 \%$ tensile strain required for failure.

The balance between avoiding balsa shear failure while reaching face sheet failure within the actuator travel has been very difficult. This has not been achieved and, based on testing and calculations, is not possible with these materials. In order for these unflawed failures to be achieved it is recommended to use a different core material, such as a foam or honeycomb, that has a higher shear strength. While beams of these materials cannot be used for studying unflawed face sheets, the use of these beams for investigating face sheets with IP waves is discussed below.

\section{$\underline{\text { In-Plane Wave Beam }}$}

IP waves were tested in compression because compression is considered a higher priority of the beam tests than tension. This is because the effect of the core stabilization is expected to have a larger effect on compressive data than tensile due to the stabilization from buckling caused by the core. Therefore, the effect of a wave in tension in flexure will not be investigated until this has been accomplished in compression.

Beams 6 through 8 were IP wave failures in compression. The ultimate stress was $279 \mathrm{MPa}$, compared to $257 \mathrm{MPa}$ in the coupons. The strain at failure was $0.70 \%$, compared to $0.84 \%$ in the coupons. These values are similar and shows that the flawed compressive face sheet may be stiffer than the flawed coupon in compression.

From BMT, strain at failure for DFIP coupons in tension was $1.66 \%$. This is larger than tensile strains reached in any of the face sheets which was $1.32 \%$. Due to 
these strains being close, a failure in a tension side face sheet with a wave may be possible. While it seems that increasing the severity of the wave would increase the likelihood of achieving a failure, previous research found that DFIP waves, within the range investigated of 29 to 49 degree misalignment angles, have the same strain at failure (Nelson, 2013). This means that increasing the misalignment angle would not decrease strain at failure, thus not increasing the likelihood of a tensile face sheet failure.

A table of all tested beams can be seen in Table 11. This includes face sheet thicknesses, loading geometry, and test results. Whether a tensile or compressive face sheet failure was attempted is shown with $\mathrm{T}$ or $\mathrm{C}$. The last column is the failure type where FS is a face sheet failure.

Table 11: Sandwich beam face sheet and loading geometry and test results.

\begin{tabular}{|c|c|c|c|c|c|c|c|c|c|}
\hline \multirow[b]{2}{*}{ Beam \# } & \multicolumn{2}{|c|}{ Face Sheet Thickness } & \multicolumn{2}{|c|}{ Load Roller Dist } & \multirow[b]{2}{*}{ T or C Failure } & \multirow[b]{2}{*}{ Wave? } & \multirow{2}{*}{$\begin{array}{c}\text { Max Load } \\
(\mathrm{kN})\end{array}$} & \multirow{2}{*}{$\begin{array}{c}\text { Failure Side FS Strain } \\
\text { (abs \%) }\end{array}$} & \multirow{2}{*}{ Failure Type } \\
\hline & Top (mm) & Bottom $(\mathrm{mm})$ & Top $(\mathrm{cm})$ & Bottom $(\mathrm{cm})$ & & & & & \\
\hline 1 & 8 & 4 & 38.1 & 91.4 & $\mathrm{~T}$ & No & 11.8 & 0.11 & None \\
\hline 2 & 2 & 1 & 38.1 & 91.4 & T & No & 12.7 & 1.26 & Balsa Shear \\
\hline 3 & 2 & 1 & 38.1 & 101.6 & T & No & 10.3 & 1.32 & Balsa Shear \\
\hline 4 & 2 & 1 & 76.2 & 223.5 & T & No & 2.1 & 0.51 & None \\
\hline 5 & 1 & 2 & 50.8 & 157.5 & C & No & 6.2 & 1.08 & FS \\
\hline 6 & 1 & 2 & 50.8 & 157.5 & c & Yes & 4.1 & 0.68 & FS in wave \\
\hline 7 & 1 & 2 & 50.8 & 157.5 & c & Yes & 4.4 & 0.74 & FS in wave \\
\hline 8 & 1 & 2 & 50.8 & 157.5 & C & Yes & 3.8 & 0.68 & FS in wave \\
\hline
\end{tabular}

\section{$\underline{\text { Sandwich Beam Conclusion }}$}

Sandwich beam testing in four-point bending is an important next step in increasing scale in the test pyramid in Figure 2 above. The work herein is developing this test specimen for future testing along with a brief investigation of IP waves in compression. The goals were to design a beam which could undergo unflawed 
compressive and tensile face sheet failures as well as flawed compressive and tensile face sheet failures.

In summary, sandwich beams made with these materials cannot result in unflawed compression or tension face sheet failures. Compression face sheet failures with a 29 degree DFIP wave can be achieved and correlates well to a similar size flaw on the coupon scale. It may be possible that a tension face sheet failure with a DFIP wave can also be achieved.

Future work includes obtaining the failures not included herein as well as more tests on beams with flawed compression face sheets. The failures not achieved include a flawed tension face sheet failure which may be possible with the given materials. Also included are unflawed compression and tension face sheet failures which likely necessitate a stronger core material. Also, more testing on the flawed compression face sheet beams should be performed to further bridge the gap between the coupon and fullscale wind turbine level. The flaw could also be a CFIP wave to investigate partial flaws and be part of the future work for the CFIP waves by testing more severe CFIP waves.

Lastly, beam manufacturing efficiency is recommended to be increased before starting a comprehensive sandwich beam test schedule. This can include wider beams being shot at a time so that multiple beams can be cut out across the width. This could allow efficiency to double or triple if the width is increased by just 2 or 3 times. Slight manufacturing changes may be necessary to implement this, including a wider mold. Another option is reducing beam width although this is not recommended for the flawed 
beams given the effect of discontinuous fibers is expected to be larger if they are a larger proportion of total fibers. 


\section{CHAPTER 6}

\section{CONCLUSIONS AND RECOMMENDED FUTURE WORK}

This work has been investigating the effects of defects from multiple angles. The work has included multiple scales of testing as well as FE modeling. Coupon-level testing investigated partial flaw in-plane waves to bridge the gap between previous extensive coupon testing and future sandwich beam testing. The coupon testing was supported with modeling which also served to improve the robustness of a previously developed combined FE modeling method. Lastly, multiple sandwich beam tests were performed in order to develop the sandwich beam for future testing of effects of defects in flexure as well as to investigate the effect of scale on the effect of in-plane waves.

The conclusions that can be drawn from this work are as follows:

1. The location of damage in CFIP coupons was near the fiber maximum misalignment angle. The severity of the damage was proportional to the degree of misalignment, as seen in the DIC data. This was also supported with the damage of the cohesive elements in the model first damaging in the region of maximum misalignment and spreading to less misaligned

locations as loading continued. Previous findings have shown that damage caused by fiber waviness was shear dominated which, is in part, further substantiated herein while also built upon given the complexity of this wave as discussed below. 
2. For constant amplitude waves, matrix damage and failure in testing and modeling was shear dominated. However, with the varying misalignment of the CFIP wave, transverse stresses became non-negligible. This was shown in the model with cohesive element damage and failures occurring in locations of both high shear stress and high transverse stress. This was further substantiated with the spatial correlation between visible matrix cracking and the positive transverse strain field measured with the DIC system. The effects of transverse strains in progressive damage become significant in waves with changing wave severity which is of importance because waves with changing severity are most common.

3. The CFIP coupons tested had a much more similar response, both qualitatively and quantitatively, to control coupons than did DFIP coupons. The only comparator in which CFIP were not more similar to control coupons was initial stiffness which was similar for all three types of coupon, likely due to matrix locking. While it must be kept in mind that misalignment angles were also different between wave types, partial flaws tested herein had a smaller effect on material property degradation. This means that partial IP waves, as seen in manufactured parts, can be designed with smaller safety factors based on partial flaw testing.

4. Stitching fibers in the glass mats were likely the cause of discretization of shear strain accumulation both in and out of the wave, as seen in DIC data, but not expected and not correlating analytically. The stitching may also 
have had an effect on magnitudes of shear strains caused by constructive and destructive interference.

5. Better analytical-experimental correlation was achieved after decreasing the *DAMAGE INITIATION value to correspond with damage initiation observed in testing. However, this decrease resulted in cohesive element softening before shear strain magnitudes similar to those seen in experiment were reached.

6. Accounting for crack energy release rates with the quasi 3D method resulted in good analytical-experimental correlation.

7. Stress vs. Strain curves generated analytically correlated very well with experiment. Longitudinal and transverse strain fields generated analytically also correlated well to experimental, as seen in the DIC. The shear strain field, however, varied in location and magnitude. After the updates implemented herein, the previously developed combined modeling approach was overall accurate both quantitatively and qualitatively for the more complex CFIP wave. Analytical-experimental correlation can likely be further increased as recommended with future work.

8. Processing times of the model were greatly reduced for the CFIP wave type of smaller misalignment angle compared to the DFIP wave. This was due to less cohesive element damage and deletion. Processing time was further reduced by spacing cohesive element columns $4 \mathrm{~mm}$. Given both of these factors, the processing time was reduced by $85 \%$. 
9. Flawed face sheet failure was achieved and studied in compression with a DFIP wave. The face sheet was found to have slightly larger ultimate stress and smaller strain at failure compared to previously tested compression coupons with a similar DFIP wave.

10. Flawed face sheet failure may be possible to achieve in tension with the materials used herein.

11. Unflawed tension and compression face sheet failures are not possible with the materials used for the sandwich beam herein.

The applications and limitations of the work herein are as follows:

1. From testing CFIP wave coupons and comparing to previous DFIP coupon data, it appears that similarly misaligned CFIP waves result in smaller material property degradations. Due to the CFIP waves being common in manufactured parts, safety factors based on DFIP coupons are too conservative. A limitation on this is that safety factors for CFIP type waves can only be gained from the wave severities tested. The wave severities herein were limited by the coupon width, in which misalignment angles varied from 4 to 10 degrees. Therefore, safety factors from larger misalignment angles cannot be determined without further testing which will require a new, wider test specimen.

2. It has been proven that the modeling method further developed herein adequately models the more complex CFIP wave. Given that this wave type is common in manufactured parts, this modeling method is now 
directly applicable to IP waves seen in parts. Furthermore, accuracy has been increased with the quasi 3D crack energy method. Lastly, processing time has been reduced, making this modeling method faster as well as more accessible for those with less computational power.

3. A limitation with this model moving forward will be the size of test specimen, especially if moving up in scale to the sandwich beams. Given the method of mesh generation used herein, mesh refinement may need to be implemented to keep processing times low.

4. Due to the increased applicability of flexural beam tests to wind turbine blades, the flawed compression face sheet testing performed herein can be directly applied. This will allow for more representative safety factors.

5. Comparing flawed sandwich beam tests to the coupon level was limited by strain measurement systems. The DIC system was too large to fit within the load frame, requiring strain to be measured with strain gages. This did not allow for full-field strain to be captured as was measured for the flawed compressive coupons.

6. A limitation on the sandwich beam development was the materials used. The balsa core shear strength was not large enough to allow for unflawed face sheet failures.

7. Another limitation on sandwich beam testing is the production rate of the beam specimens. The manufacturing process is currently so involved that a 
large number of tests necessary for a comprehensive study may not be feasible.

In order to build on the work herein, the following list of future work is included:

1. Investigation of the CFIP wave at larger misalignment angles is recommended. While the results were consistent over the small range of angles investigated, these angles were on the small side of IP waves seen in wind turbine blades. Larger waves were not tested due to the limitation caused by the small width, as discussed above. While wider coupons may be possible, larger misalignment angles in CFIP waves can be implemented on the wider sandwich beams developed herein. Along with larger waves in CFIP test specimens, the DFIP waves can be made smaller on the coupon scale to allow for direct comparisons.

2. The complexity of the CFIP coupons necessitates further experimental investigation into wave characterization. Due to misalignment angle gradient across the width, damage and failure effects of transverse strain were introduced which have not been investigated in previous research on the DFIP wave. While the wave parameter affecting shear strain is misalignment angle, the parameter affecting transverse strain is unknown. This work would likely designate another wave critical flaw parameter along with or in conjunction with the misalignment angle. Two of the many variables that could possibly affect transverse strain are the rate of change of misalignment angle across the width and the ratio of straight fiber tows 
on edge of the wave to fiber tows in wave. The effect of straight fibers is expected to increase transverse stiffness, prohibiting horizontal fiber displacements, thus altering the load redistribution observed herein. This investigation could also include determining the cause of failure initiation location in the maximum misalignment of the wave or in the left side straight fiber tows.

3. The CFIP wave has been investigated experimentally and analytically in static tension in the work herein. This can be expanded upon with static compression as well as fatigue. Further, all of these loading types can be performed on coupons with waves not centered down the length of the coupon. This would result in an asymmetric loading state and would allow for insight into the effect of location and corresponding loading state on the response of a wave in a manufactured part. Taking this to the next step, non-uniaxial testing can be implemented.

4. Investigation of the progressive damage at various loading states is recommended. This can be accomplished by pausing tests and removing coupons to image fracture surfaces. Micrographs can be produced with a scanning electron microscope.

5. Investigation into the shear strain bands observed in the DIC data is deemed worthwhile future work in order to determine the validity of DIC shear strain data. Determination of the shear bands based on stitching can be investigated by using glass mats with different stitching spacing or 
removing the stitching in the glass mats used herein before lay-up. DIC shear bands presence or alignment with new stitching spacing could determine whether these were caused by the stitching. The shear strain magnitudes should also be compared to determine if the shear bands have an effect on magnitude.

6. As mentioned above, the *DAMAGE EVOLUTION parameters for the model may be able to implement a more exact traction-separation criteria resulting in better shear strain correlation. This would require using the tabular keyword. The exact traction-separation curves could be generated analytically, as done above, and requiring a 3D model for Mode II loading. These could also be obtained experimentally, perhaps resulting in even better correlation.

7. The possibility of predictivity in regards to $* D A M A G E$ INITIATION can be investigated. This can be performed by making analytical-experimental comparisons with CFIP wave coupons over a larger range of misalignment angles. If correlation is high, without the need for tuning ${ }^{*} D A M A G E$ INITIATION values, then this parameter is considered predictive, as desired.

8. It is recommended to determine *DAMAGE INITIATION values using a more exact method than based on DIC data. One possibility is using acoustic emissions to determine when damage has initiated.

9. Now that the sandwich beam has been developed, flawed compressive face sheet beam tests can be performed. While a number of these have been 
performed with DFIP waves, CFIP waves can now be employed. The partial waves will build on the coupon partial wave work herein and can be applied more directly to flaws in wind turbine blades.

10. A flawed tension face sheet failure is likely possible. This failure should be attempted and followed by a comprehensive test program.

11. A stronger core material can be implemented, allowing for unflawed compression and tension face sheet failures to be achieved. This would allow a baseline for the sandwich beam to be developed. 


\section{REFERENCES CITED}




\section{REFERENCES CITED}

1. Abaqus Software and Abaqus Documentation: v. 6.12; Dassault Systemes Simulia Corp, Providence, RI.

2. Adams, D., \& Bell, S. (1995). Compression Strength Reductions in Composite Laminates Due to Multiple-Layer Waviness. Composites Science and Technology 53.2, 207-212.

3. Adams, D., \& Hyer, M. (1993). Effects of Layer Waviness on the Compression Strength of Thermoplastic Composite Laminates. Journal of Reinforced Plastics and Composites 12.4, 414-429.

4. American Wind Energy Association. (2013). 2012 U.S. Wind Industry Annual Market Report. Washington, DC.

5. Aramis Digital Image Correlation Software and Documentation: v 6.2; Trilion Quality Systems, Plymouth Meeting, PA.

6. ASTM D3518. Standard Test Method for In-Plane Shear Response of Polymer Matrix Composite Materials by Tensile Test.

7. ASTM D5528-13. Standard Test Method for Mode I Interlaminar Fracture Toughness of Unidirectional Fiber-Reinforced Polymer Matrix Composites.

8. ASTM D6272. Standard Test Method for Flexural Properties of Unreinforced and Reinforced Plastics and Electrical Insulating Materials by Four-Point Bending.

9. ASTM WK22949. New Test Method for Determination of the Mode II Interlaminar Fracture Toughness of Unidirectional Fiber-Reinforced Polymer Matrix Composites Using the End-Notched Flexure (ENF) Test.

10. Avery, D. P. (2004). Compression Strength of Carbon Fiber Laminates Containing Flaws with Fiber Waviness. 2004 ASME Wind Energy Symposium, ASME/AIAA. 174. AIAA.

11. Barbero, E. J. (2011). Introduction to Composite Materials Design (2 ed.). Boca Raton, FL: CRC Press.

12. Birks, N., Meier, G. H., \& Pettit, F. S. (2006). Introduction to the HighTemperature Oxidation of Metals. New York: Cambridge University Press.

13. Cairns, D., Riddle, T., \& Nelson, J. (2011). Wind Turbine Composite Blade Manufacturing: The Need for Understanding the Defect Origins, Prevalence, Implications and Reliability. Sandia National Laboratories SAND2011-1094. 
14. Camanho, P., \& Matthews, F. (1999). A Progressive Damage Model for Mechanically Fastened Joints in Composite Laminates. Journal of Composite Materials 33.24, 2248-2280.

15. Cargo Composites. (2014). AeroPlaz Honeycomb Core Composite. Retrieved from Cargo Composites: http://cargocomposites.com/technology/

16. Composites World. (2014, 11 17). MHI Vestas Offshore Wind to Start Production of $80 \mathrm{~m}$ Blades by $2 Q 2015$. Retrieved from Composites World:

http://www.compositesworld.com/news/mhi-vestas-offshore-wind-to-startproduction-of-80m-blades-by-2q-2015

17. CompositesWorld. (2014). Sandwich Cores for Closed Molding. Retrieved from CompositesWorld: www.compositesworld.com

18. Daniel, I., \& Abot, J. (2000). Fabrication, Testing and Analysis of Composite Sandwich Beams. Composites Science and Technology 60, 2455-2463.

19. Daniel, I., Gdoutos, E., Wang, K., \& Abot, J. (2002). Failure Modes of Composite Sandwich Beams. International Journal of Damage Mechanics, 11, 309-334.

20. Davila, C., Camanho, P., \& Rose, C. (2005). Failure Criteria for FRP Laminates. Journal of Composite Materials, 323 - 345.

21. Department of Energy. (July 2008). $20 \%$ Wind Energy by 2030: Increasing Wind Energy's Contribution to US Electricity Supply. NREL. DOE/GO-102008-2567.

22. Gebert, E. (2012, 6 1). Broken Turbine Blad Problem Found. Retrieved from Times Bulletin: http://timesbulletin.com/Content/News/News/Article/Brokenturbine-blade-problem-found/2/4/173854

23. Gibson, R. (2011, January). A Mechanics of Materials/Fracture Mechanics Analysis of Core Shear Failure in Foam Core Composite Sandwich Beams. Journal of Sandwich Structures and Materials, 13, 83-95.

24. Hayman, B., Berggreen, C., \& Pettersson, R. (2005). The Influence of Face Sheet Wrinkle Defects on the Performance of FRP Sandwich Structures. Sandwich Structures: Advancing with Sandwich Structures and Materials, 7, 393-402.

25. Hayman, B., Berggreen, C., \& Pettersson, R. (2007, July). The Effect of Face Sheet Wrinkle Defects on the Strength of FRP Sandwich Structures. Journal of Sandwich Structures and Materials, 9, 377-404.

26. Hexion. (2006). Hexion Technical Data Sheet. EPIKOTE Resin MGS RIMR 135 and EPIKURE Curing Agent MGS RIMH 134-RIMH 137. 
27. Kassapoglou, C. (2013). Design and Analysis of Composite Structures (2 ed.). West Sussex, UK: Wiley and Sons, Ltd.

28. Manalo, A., Aravinthan, T., \& Karunasena, W. (2010, October). Flexural Behaviour of Glue-Laminated Fibre Composite Sandwich Beams. Composite Structures, 92, 2703-2711.

29. Manalo, A., Aravinthan, T., Karunasena, W., \& Islam, M. (2010). Flexural Behaviour of Structural Fibre Composite Sandwich Beams in Flatwise and Edgewise Positions. Composite Structures, 984-995.

30. MIL-HDBK-17-1F: Composite Materials Handbook, Volume 1. Polymer Matrix Composites - Guidelines for Characterization of Structural Materials, U.S. Department of Defense, 2002.

31. Narciso, M. L. (1999). Probabilistic Design Methodology for Composite Aircraft Structures. Northrup Grumman Corp Dallas TX Commercial Aircraft Div.

32. Nelson, J. (2013). A Comparison of Continuum and Discrete Modeling Techniques of the Effects of Manufacturing Defects Common to Composite Structures. Industrial and Mechanical Engineering, Montana State University Composites Group. Bozeman: Montana State University.

33. Paquette, J. (2012). Blade Reliability Collaborative (BRC). Sandia National Laboratories, Wind Energy Technology Dept. Retrieved from http://energy.sandia.gov/wp/wp-content/gallery/uploads/3-B-3-PaquetteSAND2012-5566C.pdf

34. Philippidis, D. L. (2008). Mechanical Property Variability in FRP Laminates and its Effect on Failure Prediction. Composites Part B: Engineering 39.7, 1247-1256.

35. Reinforced Plastics. (2014, 2 19). An Introduction to Core Materials: Part 1Designing Sandwich Structures. Retrieved from Reinforced Plastics: www.reinforcedplastics.com

36. Riddle, W. (2013). Development of Reliability Program for Risk Assessment of Composite Structures Treating Defects as Uncertainty Variables. Industrial and Mechanical Engineering, Montana State University Composites Group. Bozeman: Montana State University.

37. Sandia National Laboraties. (2014, 6 10). Blade Reliability Collaborative. Retrieved from Sandia National Laboratories Energy and Climate: http://energy.sandia.gov/energy/renewable-energy/wind-power/materialsreliability-standards/blade-reliability-collaborative/ 
38. Siemens. (2012, August). B75 Rotor Blade Fact Sheet. Retrieved from Siemens: http://www.siemens.com/press/pool/de/feature/2012/energy/factsheet-b75-rotorblade-e.pdf

39. Siemens. (2012, 8 6). Record-Size Rotor Blades Transported to Destination. Retrieved from Siemens Global Website: http://www.siemens.com/innovation/en/news/2012/e_inno_1226_2.htm

40. Staff. (2010). Suzlon Blade Recall: Retrofit Program to Address Cracking. Composites World.

41. Trilion. Aramis. Retrieved from Trilion Quality Systems: http://trilion.com/3dsoftware/aramis/\#

42. Wang, J. (2012). Experimental Fabrication and Characterization of Out-of-Plane Fiber Waviness in Continuous Fiber-Reinforced Composites. Journal of Composite Materials 46.17, 2041-2053.

43. what-when-how. (2014). Distributed Fiber Optic Strain Measurement Using Rayleigh Scatter in Composite Structures (Experimental and Applied Mechanics). Retrieved from what-when-how: http://what-when-how.com/experimental-andapplied-mechanics/distributed-fiber-optic-strain-measurement-using-rayleighscatter-in-composite-structures-experimental-and-applied-mechanics/

44. Wisnom, M. (1994, January). The Effect of Fibre Waviness on the Relationship Between Compressive and Flexural Strengths of Unidirectional Composites. Journal of Composite Materials, 28, 66-76.

45. Wu, Y.; Shivpuri, R.; Lee, L.J. (1998). Effect of Macro and Micro Voids on Elastic Properties of Polymer Composites. Journal of Reinforced Plastics and Composites, 1391-1402. 
APPENDICES 
$\underline{\text { APPENDIX A }}$

ABAQUS INPUT FILE 
*node, nset=global

$1, \quad 0.000000, \quad 50.000000,0.000000$

$2, \quad 0.500000, \quad 50.000000, \quad 0.000000$

$3, \quad 1.000000, \quad 50.000000,0.000000$

4, $1.500000, \quad 50.000000, \quad 0.000000$

$5, \quad 2.000000, \quad 50.000000, \quad 0.000000$

$6, \quad 2.500000, \quad 50.000000,0.000000$

$7, \quad 3.000000, \quad 50.000000,0.000000$

$8, \quad 3.500000, \quad 50.000000, \quad 0.000000$

$9, \quad 4.000000, \quad 50.000000, \quad 0.000000$

$10, \quad 4.500000, \quad 50.000000,0.000000$

40192, $\quad 46.000000, \quad-50.000000, \quad 0.000000$

$40193, \quad 46.500000, \quad-50.000000,0.000000$

$40194, \quad 47.000000, \quad-50.000000,0.000000$

$40195, \quad 47.500000, \quad-50.000000,0.000000$

$40196, \quad 48.000000, \quad-50.000000,0.000000$

$40197, \quad 48.500000, \quad-50.000000,0.000000$

$40198, \quad 49.000000, \quad-50.000000,0.000000$

$40199, \quad 49.500000, \quad-50.000000,0.000000$

$40200, \quad 50.000000, \quad-50.000000, \quad 0.000000$

$40201, \quad 25.000000, \quad 50.000000, \quad 0.000000$

$40202, \quad 25.000000, \quad-50.000000,0.000000$

*element, type $=$ cps 4 ,elset $=$ conventional 4

$1,1,101,102,2$

$2, \quad 2, \quad 102,103,3$

$3,3,103,104,4$

$4, \quad 4, \quad 104,105,5$

$5,5,105,106,6$

$6,6,106,107,7$

$7, \quad 7, \quad 107, \quad 108,8$

$8, \quad 8, \quad 108, \quad 20208,20108$

$9, \quad 9, \quad 109, \quad 110,10$

$10, \quad 10, \quad 110,111,11$

9991, 9991, 10091, 10092, 9992

9992, 9992, 10092, 10093, 9993

9993, 9993, 10093, 10094, 9994

9994, 9994, 10094, 10095, 9995

9995, 9995, 10095, 10096, 9996 
9996, 9996, 10096, 30196, 30096

9997, 9997, 10097, 10098, 9998

9998, 9998, 10098, 10099, 9999

9999, 9999, 10099, 10100, 10000

10000, 10000, 10100, 30200, 30100

*element, type $=\operatorname{coh} 2 \mathrm{~d} 4$, elset $=$ cohesive 10001, 20108, 20208, 109, 9, 10002, 20116, 20216, 117, 17, 10003, 20124, 20224, 125, 25, 10004, 20132, 20232, 133, 33, 10005, 20140, 20240, 141, 41, 10006, 20148, 20248, 149, 49, 10007, 20156, 20256, 157, 57, 10008, 20164, 20264, 165, 65, 10009, 20172, 20272, 173, 73, 10010, 20180, 20280, 181, 81,

11191, 30024, 30124, 10025, 9925, 11192, 30032, 30132, 10033, 9933, $11193,30040,30140, \quad 10041,9941$, 11194, 30048, 30148, 10049, 9949, $11195, \quad 30056,30156, \quad 10057,9957$, 11196, 30064, 30164, 10065, 9965, 11197, 30072, 30172, 10073, 9973, $11198,30080,30180,10081,9981$, $11199, \quad 30088, \quad 30188, \quad 10089,9989$, 11200, 30096, 30196, 10097, 9997, *ELSET, ELSET=P1000001 1

*SOLID SECTION, ELSET=P1000001, MATERIAL=COMP, ORIENTATION=O1000001

4

*ORIENTATION, NAME=O1000001

$0,-2,0,2,0,0$

3,0

*ELSET, ELSET=P1000002

2

*SOLID SECTION, ELSET=P1000002, MATERIAL=COMP, ORIENTATION=O1000002 


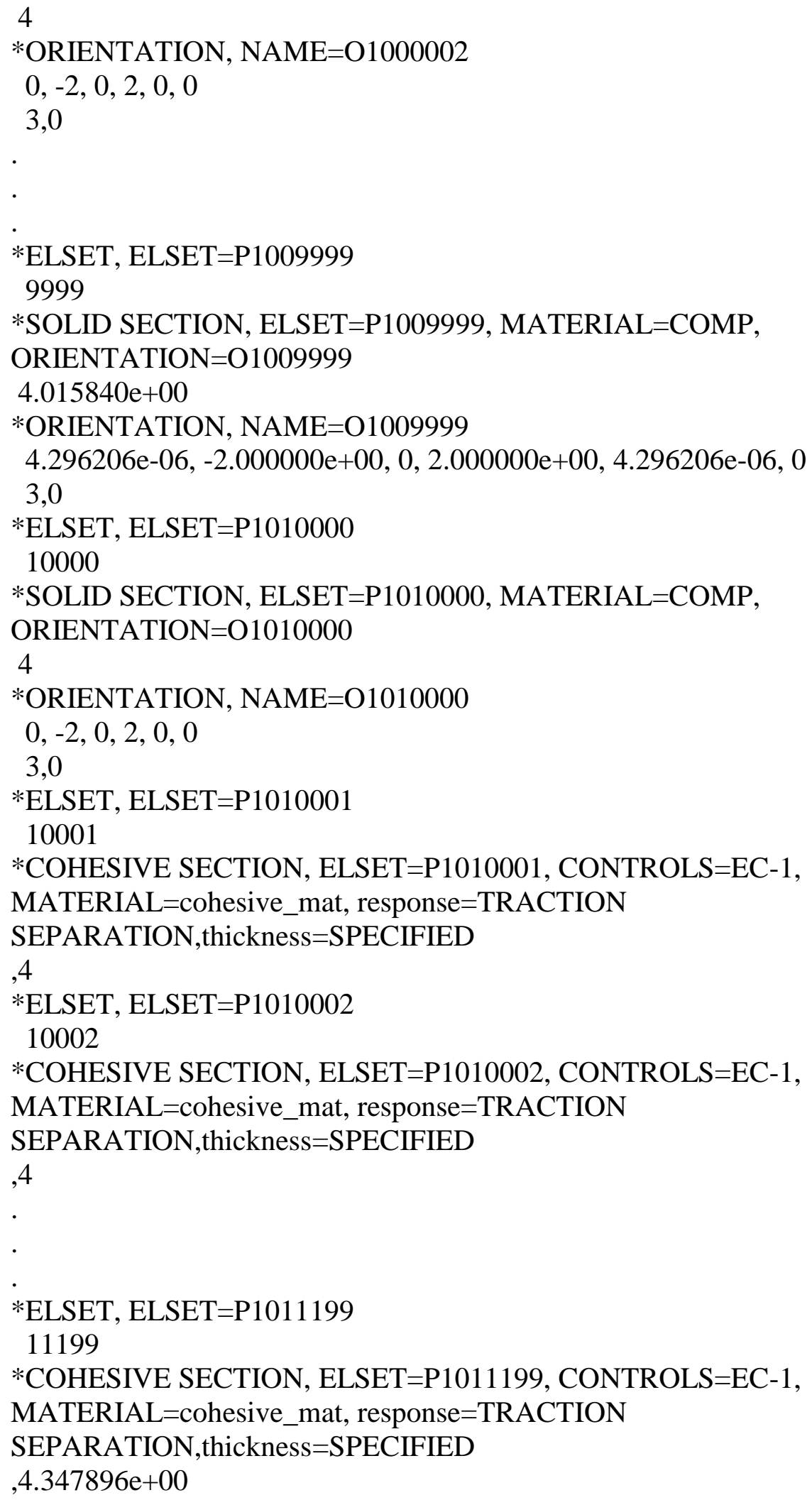




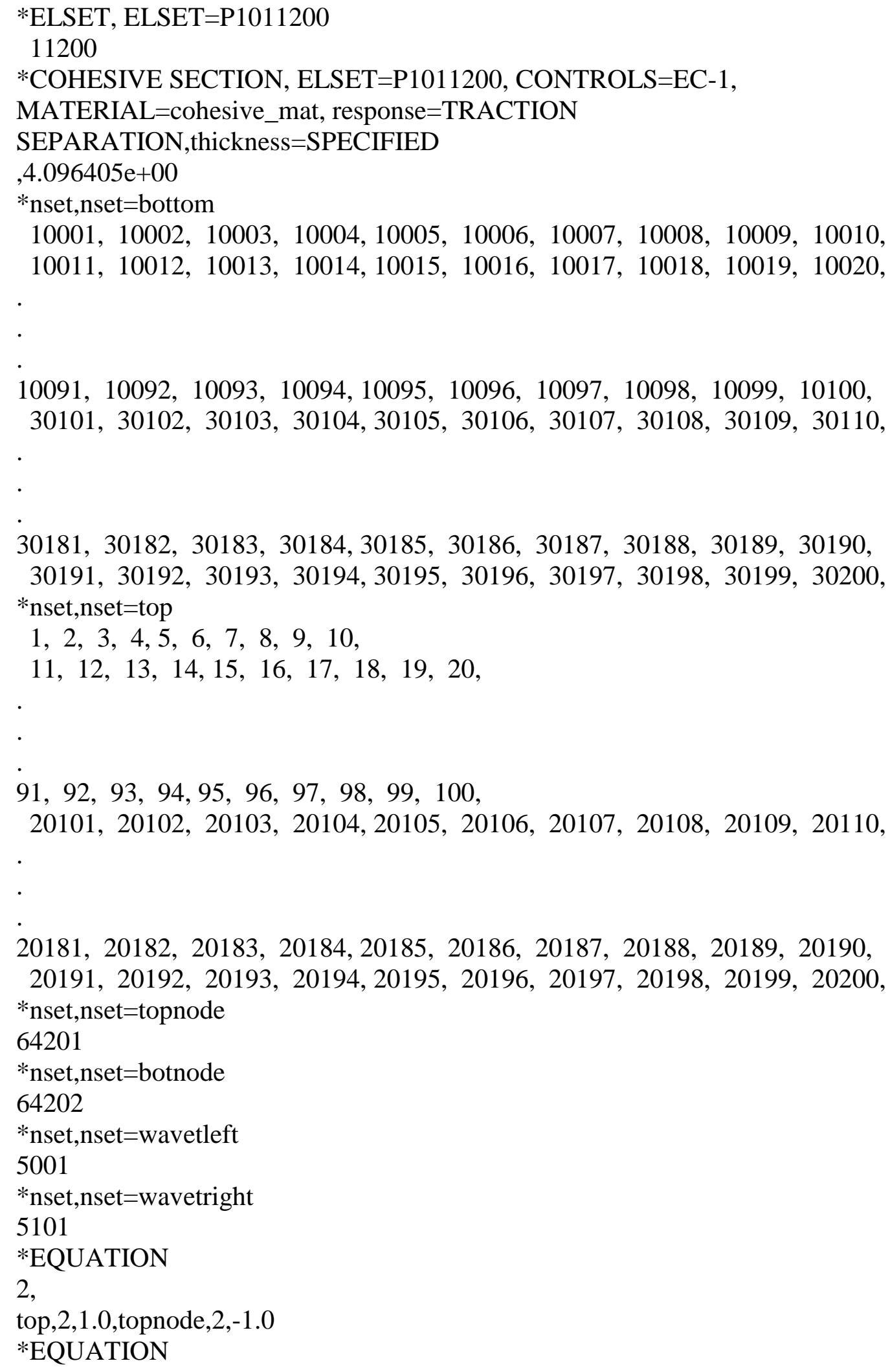

20181, 20182, 20183, 20184, 20185, 20186, 20187, 20188, 20189, 20190, 20191, 20192, 20193, 20194, 20195, 20196, 20197, 20198, 20199, 20200, *nset,nset=topnode 64201

*nset,nset=botnode

64202

*nset,nset=wavetleft

5001

*nset,nset=wavetright

5101

*EQUATION

2 ,

top, 2,1.0,topnode, 2,-1.0

*EQUATION 
2 ,

top, 1,1.0,topnode, $1,-1.0$

*EQUATION

2 ,

bottom,2,1.0,botnode, $2,-1.0$

*MATERIAL, NAME=comp

*user material,constants $=20$

40600.,4500.,0.27,16800., 0.002,0.003,0.0056,0.0091,

$0.0159,0.024,0.0356,0.0454, \quad 25,53,70,83$,

$96,103,103,103$

**

*Section Controls, name=EC-1, ELEMENT DELETION=YES, MAX

DEGRADATION=1., VISCOSITY=1e-05

$1 ., 1 ., 1$.

$* *$

*Material, name $=$ cohesive_mat

*Damage Initiation, criterion=MAXS

$60,60,0$.

*Damage Evolution, type=ENERGY, mixed mode behavior $=\mathrm{BK}$, power=2.284

3.092,3.810,0

*Elastic, type $=$ TRACTION

2.2E6,2.2E6,2.2E6

*restart,write, frequency $=10000$

** Load Step 1

*STEP, INC=10000,nlgeom

Untitled

*STATIC,stabilize $=1 . \mathrm{e}-8$

0.01,1.,1.e-9,0.05

*boundary

global,3

botnode, 2,0 .

topnode, 1,0 .

topnode, $2,1.2$

$* * *$ boundary,type $=$ displacement

**top, $1,, 4$.

$*$ Output, field,frequency $=1$

*Node Output

CF, RF, U

*Element Output, directions=YES

LE, PE, PEEQ, PEMAG, S, STATUS, SDEG

$*$ Output, history, frequency $=1$

*Node Output, nset=top

RF2, U2 
*Node Output, nset=topnode

RF2, U2

*Node Output, nset=wavetright

RF2, U2

*Node Output, nset=wavetleft

RF2, U2

*Node print, nset=topnode

RF2, U2

*Node print, nset=wavetleft

RF2, U2

*Node print, nset=wavetright

RF2, U2

*controls,parameter=field,field=displacement $0.01,1$.

*END STEP 


\section{$\underline{\text { APPENDIX B }}$}

MATLAB CODE FOR ABAQUS INPUT FILE GENERATION 


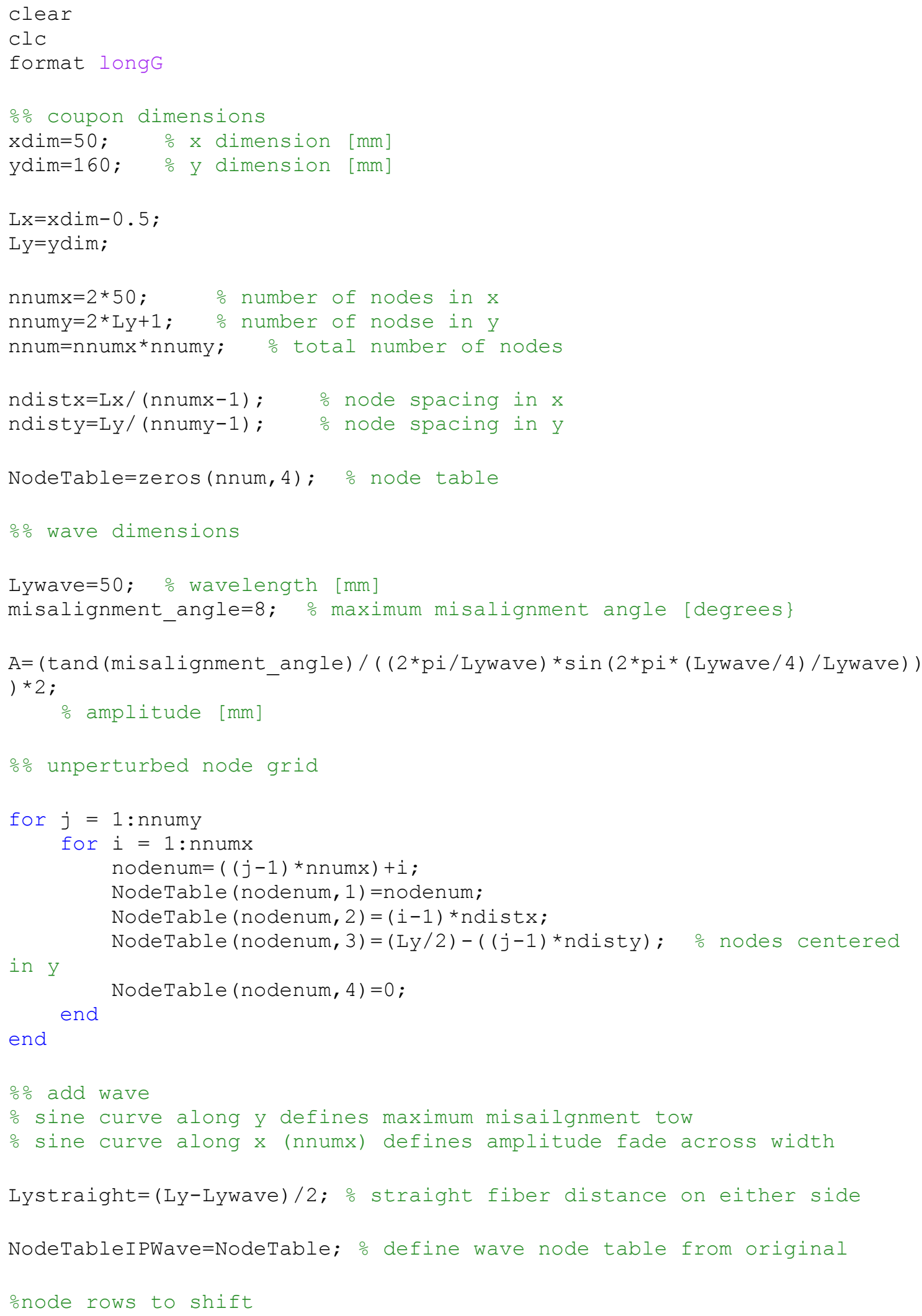




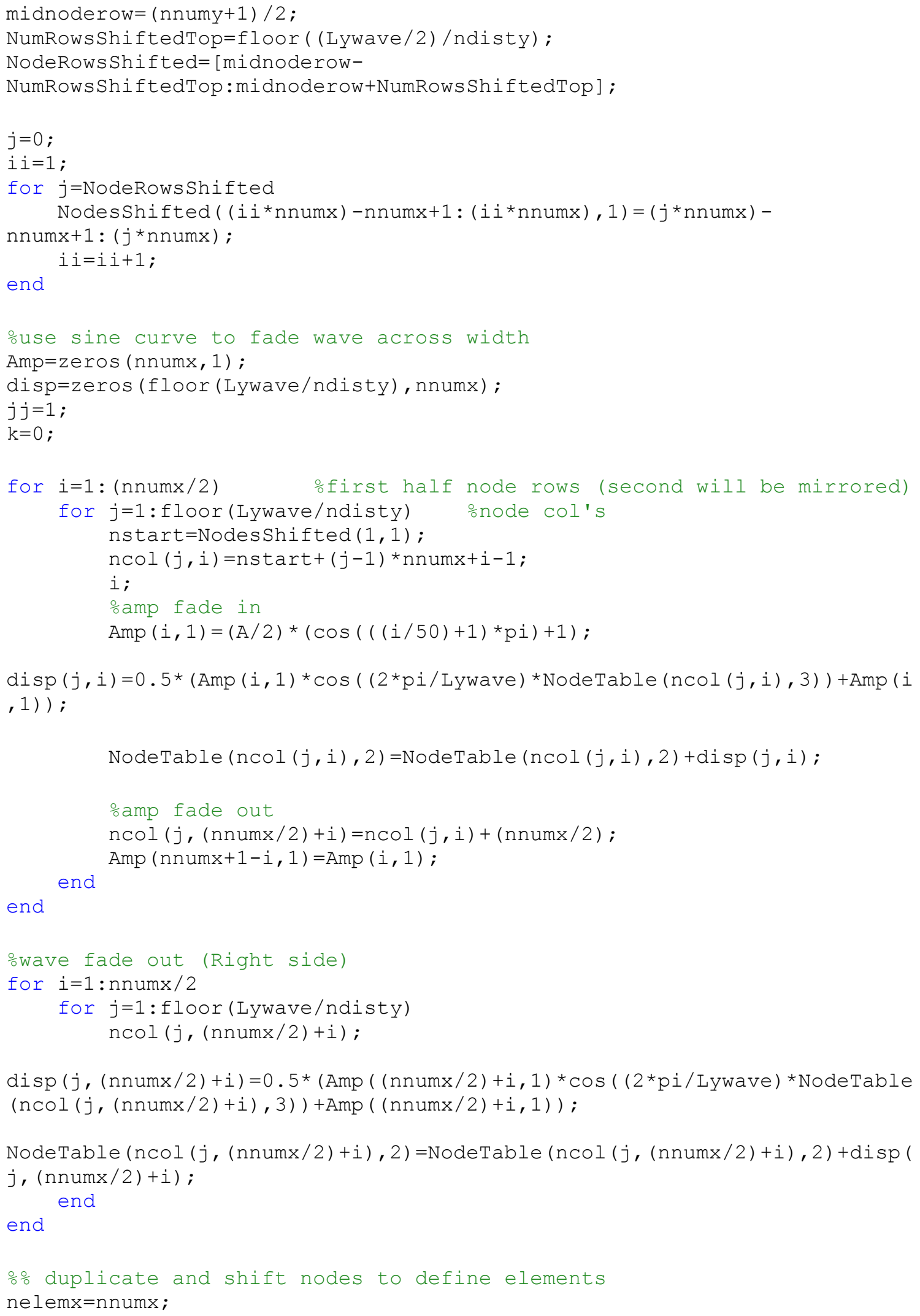




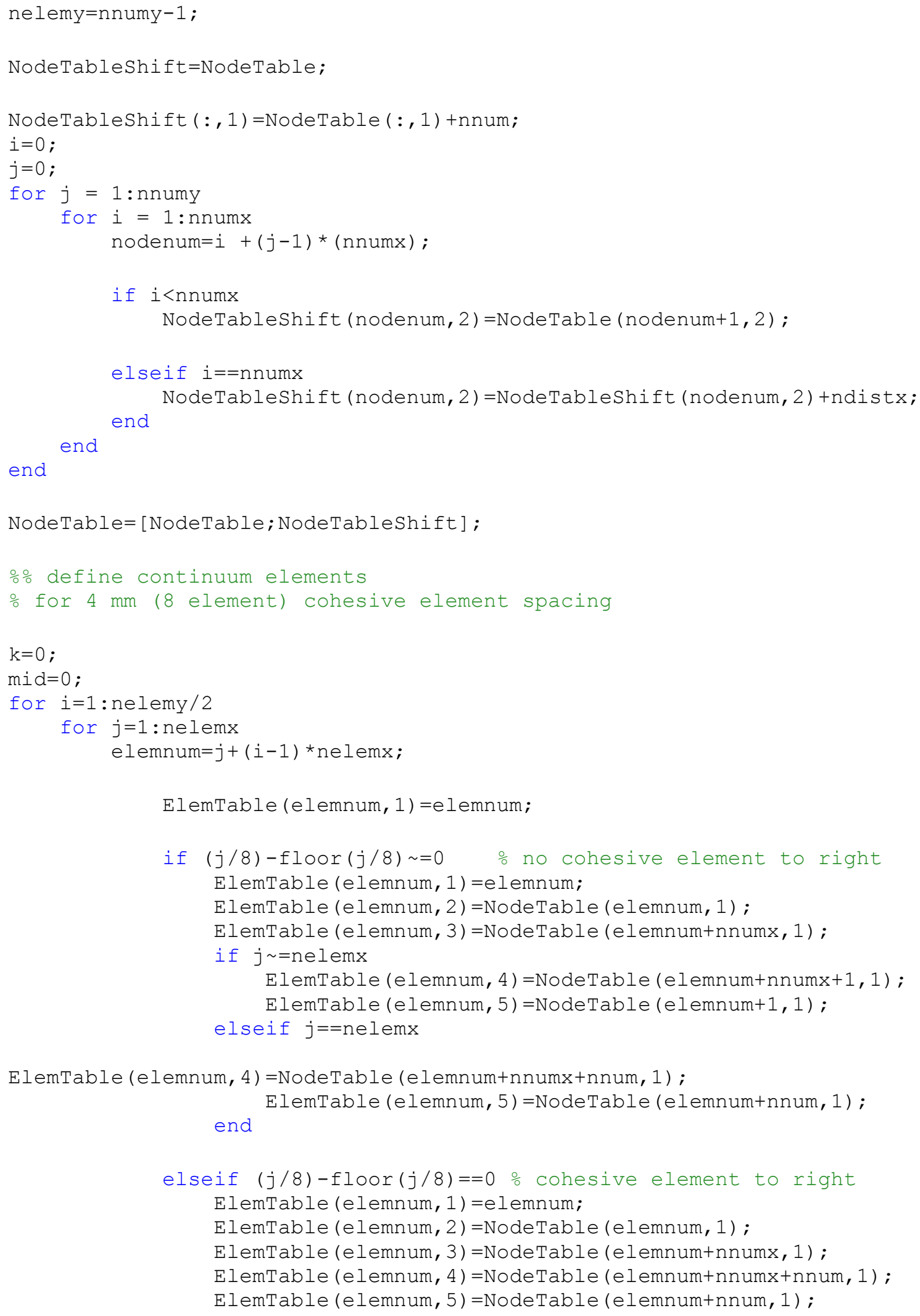


end

end

$\mathrm{k}=\mathrm{k}+1$;

$\operatorname{mid}=0$;

end

\% write continuum element table

fid=fopen ('twodipw_contf_Elements.inp', 'w') ;

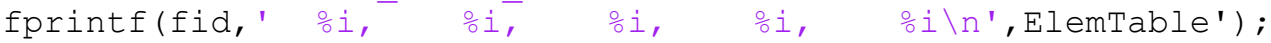

fclose (fid);

응 calculate thickness

o calculated from cross sectional area and unit volume

$t=4 ; \quad$ ofar field thickness [mm]

$\mathrm{V}=$ ndist ${ }^{*}$ ndisty ${ }^{\star}$; $\quad$ \% $\left[\mathrm{mm}^{\wedge} 3\right]$

for $j=1:$ nelemy $/ 2$

for $i=1$ : nelemx

elemnum $=i+(j-1) *$ nelemx;

$\mathrm{xl}$ =NodeTable (ElemTable (elemnum, 2),2);

yl=NodeTable (ElemTable (elemnum, 2), 3) ;

x2=NodeTable (ElemTable (elemnum, 3), 2);

y2=NodeTable (ElemTable (elemnum, 3), 3) ;

×3=NodeTable (ElemTable (elemnum, 4),2);

y3=NodeTable (ElemTable (elemnum, 4), 3);

$\mathrm{x} 4=$ NodeTable (ElemTable (elemnum, 5), 2);

y4=NodeTable (ElemTable (elemnum, 5), 3) ;

$\mathrm{A} 1=0.5 * \operatorname{abs}(\mathrm{x} 1-\mathrm{x} 2)$ *abs $(\mathrm{y} 1-\mathrm{y} 2)$;

$\mathrm{A} 2=\min ((\mathrm{x} 3-\mathrm{x} 1),(\mathrm{x} 4-\mathrm{x} 2)) * \operatorname{abs}(\mathrm{y} 1-\mathrm{y} 3)$;

$\mathrm{A} 3=0.5 * \operatorname{abs}(\mathrm{x} 4-\mathrm{x} 3)$ *abs $\left(\mathrm{y}^{4}-\mathrm{y}^{3}\right)$;

$\mathrm{A}=\mathrm{A} 1+\mathrm{A} 2+\mathrm{A} 3$;

thick (elemnum, 1)=elemnum; $\frac{\circ}{\circ}$ individual element thickness

thick (elemnum,2)=V/A; $\quad$ otable of all element thicknesses end

end

\% output for each element definition with local material orientations orientation Matrix

for $i=1:$ nelemx*nelemy/2

Orientation $(i, 1)=1000000+i ; \quad \frac{\circ}{\circ}$ elset name

Orientation $(i, 2)=i$;

olement \#

Orientation $(i, 3)=1000000+i ; \quad$ olset name

Orientation $(i, 4)=1000000+i ; \quad \frac{\circ}{0}$ orientation name \#

Orientation $(i, 5)=$ thick $(i, 2) ; \quad \%$ thickness

Orientation $(i, 6)=1000000+i ; \quad \frac{\circ}{0}$ local orientation name

nl=ElemTable $(i, 2)$;

n2=ElemTable $(i, 3)$;

n3=ElemTable $(i, 4)$;

n $4=$ ElemTable $(i, 5)$; 
$\mathrm{n} 1 \mathrm{x}=$ NodeTable $(\mathrm{n} 1,2)$;

$\mathrm{n} 1 \mathrm{y}=$ NodeTable $(\mathrm{n} 1,3)$;

$\mathrm{n} 1 \mathrm{z}=$ NodeTable $(\mathrm{n} 1,4)$;

$\mathrm{n} 2 \mathrm{x}=$ NodeTable $(\mathrm{n} 2,2)$;

$\mathrm{n} 2 \mathrm{y}=$ NodeTable $(\mathrm{n} 2,3)$;

$\mathrm{n} 2 \mathrm{z}=$ NodeTable $(\mathrm{n} 2,4)$;

$\mathrm{n} 3 \mathrm{x}=$ NodeTable $(\mathrm{n} 3,2)$;

n3y=NodeTable $(n 3,3)$;

n3z=NodeTable $(\mathrm{n} 3,4)$;

$\mathrm{n} 4 \mathrm{x}=$ NodeTable $(\mathrm{n} 4,2)$;

$\mathrm{n} 4 \mathrm{y}=$ NodeTable $(\mathrm{n} 4,3)$;

$\mathrm{n} 4 \mathrm{z}=$ NodeTable $(\mathrm{n} 4,4)$;

$\mathrm{x} 1=((\mathrm{n} 1 \mathrm{x}+\mathrm{n} 4 \mathrm{x}) / 2) ;$

$\mathrm{y} 1=((\mathrm{n} 1 \mathrm{y}+\mathrm{n} 4 \mathrm{y}) / 2)$;

$\mathrm{x} 2=((\mathrm{n} 2 \mathrm{x}+\mathrm{n} 3 \mathrm{x}) / 2)$;

$\mathrm{y}^{2}=((\mathrm{n} 2 \mathrm{y}+\mathrm{n} 3 \mathrm{y}) / 2)$;

$\mathrm{axm}=\mathrm{x} 2-\mathrm{x} 1$

a $y m=y 2-y 1$;

$\operatorname{amag}=\left((\operatorname{axm} \wedge 2)+(\operatorname{aym})^{\wedge} 2\right)$;

$\mathrm{ax}=\mathrm{axm} / \mathrm{amag}$;

$\mathrm{ay}=\mathrm{aym} / \mathrm{amag}$;

$\mathrm{bx}=-\mathrm{ay} ;$

by=ax;

angle $(i, 1)=\operatorname{atand}((x 2-x 1) /(y 1-y 2))$;

width $(i, 1)=((n 4 x-n 1 x)+(n 3 x-n 2 x)) / 2$;

\begin{tabular}{|c|c|c|}
\hline Orientation $(i, 7)=a x$; & 잉 local & orientation definition \\
\hline Orientation $(i, 8)=a y$; & local & orientation definition \\
\hline Orientation $(i, 9)=0 ;$ & local & orientation definition \\
\hline entation $(i, 10)=\mathrm{bx}$; & local & orientation definition \\
\hline entation $(i, 11)=$ by ; & local & orientation d \\
\hline entation $(i, 12)=0 ;$ & local & orientation \\
\hline
\end{tabular}

end

․ write orientation file

fid=fopen ('twodipw contf Orientation.inp', 'w' ) ;

fprintf(fid, '*ELSET, ELSET=P\%i\n \%i\n*SOLID SECTION, ELSET=P\%i,

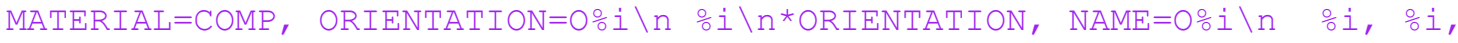

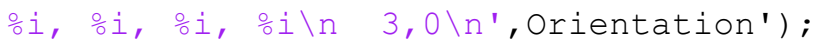

fclose (fid);

응 define cohesive elements

row $=0$;

th=8; $\% 8$ element spacing for cohesive elements (4 mm spacing)

for $j=1:$ nelemy $/ 2$

for $i=1:$ floor $($ nelemx-1)/th $)$ 


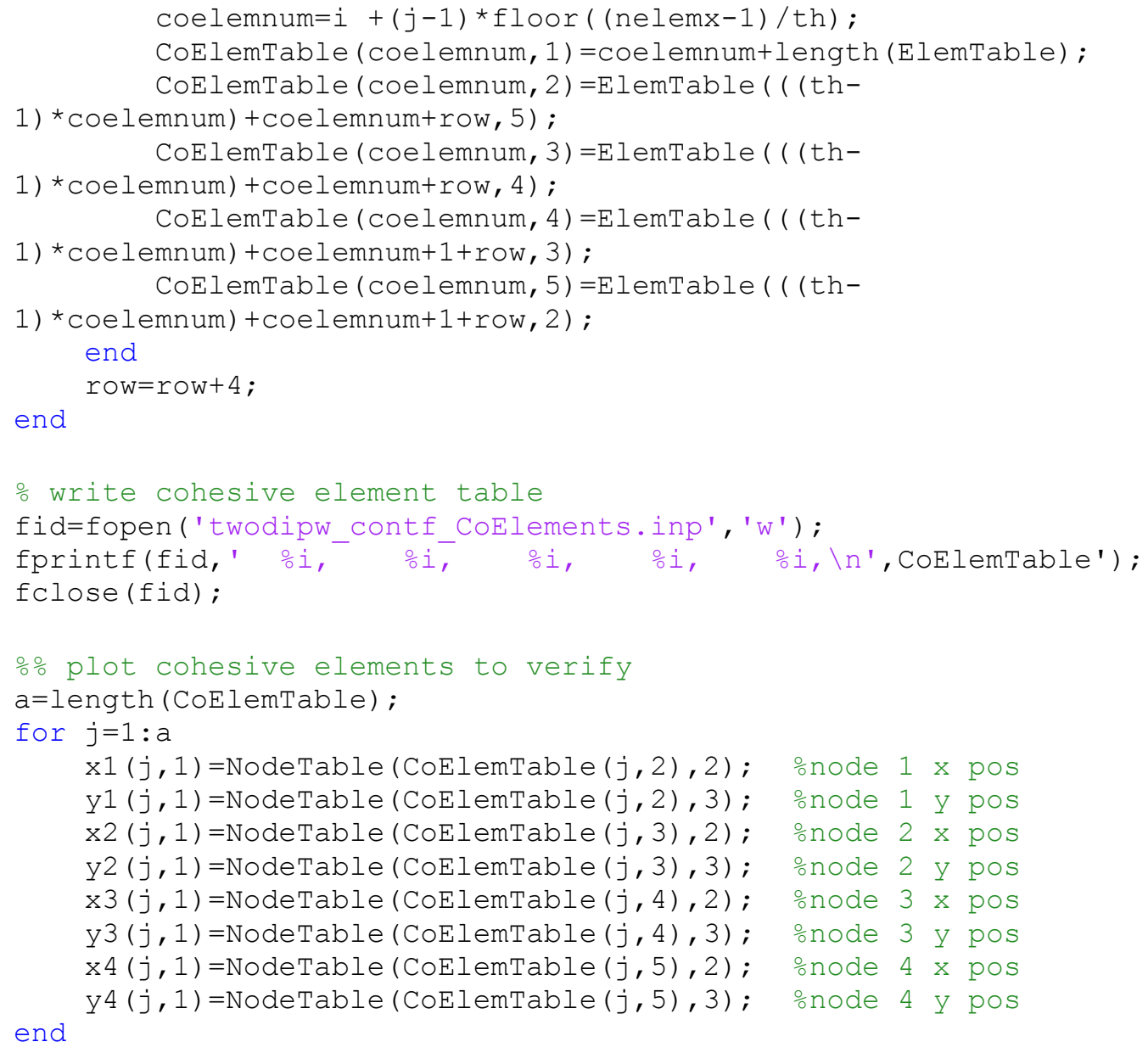


end

cotable (conum, 3) $=i * t h+(j-1) *$ nelemx +1 ;

row $=$ row +1 ;

end

for ii=1: length (cotable)

cotable $(i i, 2)=$ thick $(\operatorname{cotable}(i i, 2), 2)$;

cotable $(i i, 3)=$ thick $(\operatorname{cotable}(i i, 3), 2)$;

end

for ii=1:length (cotable)

$\cot (i \mathrm{i}, 1)=1000000+\operatorname{cotable}(i \mathrm{i}, 1) ; \quad$ \% elset \#

$\cot (i i, 2)=$ cotable $(i i, 1)$;

\% element \#

$\cot (i i, 3)=1000000+\operatorname{cotable}(i i, 1)$;

\% elset \#

$\cot (i i, 4)=(\operatorname{cotable}(i i, 2)+\operatorname{cotable}(i i, 3)) / 2$;

ㄴ thickness [mm]

end

\% write cohesive element definitions

fid=fopen ('twodipw_contf_cothick.inp', 'w') ;

fprintf(fid, '*ELSET, ELSET=P\%i\n \%i\n*COHESIVE SECTION, ELSET=P\%i,

CONTROLS=EC-1, MATERIAL=Cohesive mat, response=TRACTION

SEPARATION, thickness=SPECIFIED \n, 이 ' ', cot' ) ;

fclose (fid);

응 define node sets to be used for boundary conditions

otop node set

top $(:, 1)=[1:$ nnumx nnum+1:nnum+nnumx $]$;

fid=fopen ('twodipw_contf_topnset.inp', 'w') ;

fprintf(fid, ' $\frac{\circ}{\circ}, \frac{\circ}{\circ}$, $\frac{\circ}{\circ}, \frac{\circ}{\circ}, \frac{\circ}{\circ}, \frac{\circ}{\circ}, \frac{\circ}{\circ}, \frac{\circ}{\circ}, \frac{\circ}{\circ}$

\% $i, \backslash n^{\prime}$, top');

fclose (fid);

\% bottom node set

bottom $(:, 1)=[((($ nnumy -1$) *$ nnumx $/ 2)+1:(($ nnumy -1$) *$ nnumx $/ 2)+$ nnumx $)$

( ( (nnumy-1)*nnumx/2)+1+nnum: ( (nnumy-1)*nnumx/2)+nnumx+nnum) ] ;

fid=fopen ('twodipw_contf bottomnset.inp', 'w') ;

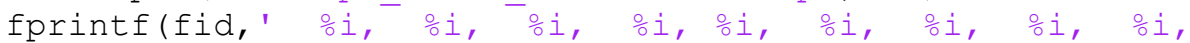

\%i, \n', bottom');

fclose (fid);

\% $\%$ add nodes to lock to node sets to define BC's and retrieve results topnode=length (NodeTable) +1

NodeTable (topnode, 1 ) =topnode;

NodeTable (topnode, 2$)=($ Lx + ndistx $) / 2$;

NodeTable (topnode, 3 ) =Ly/2;

NodeTable (topnode, 4$)=0$;

botnode=length (NodeTable $)+1$

NodeTable (botnode, 1 ) =botnode;

NodeTable (botnode, 2$)=($ Lx + ndistx $) / 2$;

NodeTable (botnode, 3 ) =-Ly/2;

NodeTable (botnode, 4$)=0$; 


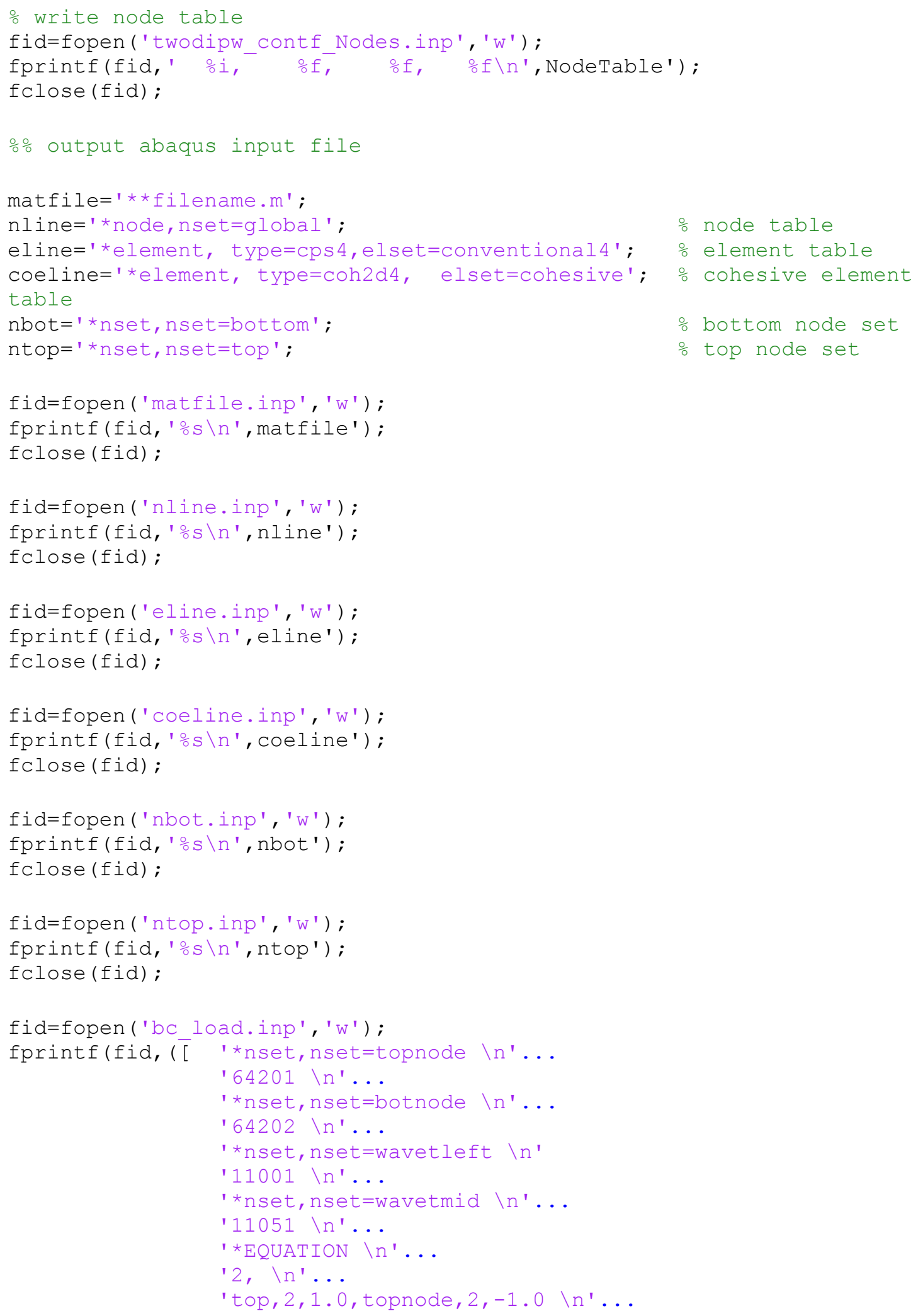


' *EQUATION $\backslash \mathrm{n}^{\prime} \ldots$

'2, $\backslash n^{\prime} \ldots$

'top, 1, 1.0, topnode, 1,-1.0 \n'...

'*EQUATION $\backslash \mathrm{n}^{\prime} \ldots$

' $2, \backslash n^{\prime} \ldots$

' bottom, 2, 1.0, botnode, 2,-1.0 \n'...

' *MATERIAL, NAME=comp $\backslash \mathrm{n}^{\prime} \ldots$

'*user material, constants $=20 \backslash \mathrm{n}^{\prime} \ldots$

'40600.,4500.,0.27,16800., 0.002,0.003,0.0056,0.0091,

$\backslash n^{\prime} \ldots$

' $0.0159,0.024,0.0356,0.0454, \quad 25,53,70,83, \quad \backslash n^{\prime} \ldots$

' $96,103,103,103 \backslash \mathrm{n}^{\prime} \ldots$

$\backslash n^{\prime} \ldots$

'*Section Controls, name=EC-1, ELEMENT DELETION=YES, MAX DEGRADATION=1., VISCOSITY=1e-05 \n'...

'1., 1., 1. $\backslash \mathrm{n}^{\prime} \ldots$

'**------------------------------------ $\backslash \mathrm{n}^{\prime} \ldots$

'*Material, name=cohesive_mat $\backslash n^{\prime} \ldots$

'*Damage Initiation, critērion=MAXS $\backslash n^{\prime} \ldots$

' $60,60,0 . \backslash n^{\prime} \ldots$

'*Damage Evolution, type=ENERGY, mixed mode

behavior=BK, power=2.284 \n'...

' $3.092,3.810,0 \backslash n^{\prime} \ldots$

'*Elastic, type=TRACTION $\backslash \mathrm{n}^{\prime} \ldots$

' $2.2 \mathrm{E} 6,2.2 \mathrm{E} 6,2.2 \mathrm{E} 6 \backslash \mathrm{n}^{\prime} \ldots$

'**---------------

-------------------------- \n'...

'*restart, write, frequency $=10000 \backslash \mathrm{n}$ '...

'** Load Step 1

$\backslash n^{\prime} \ldots$

'*STEP, INC=10000, nlgeom \n'...

'Untitled $\backslash n$ '...

'*STATIC, stabilize=1.e-8 \n' ...

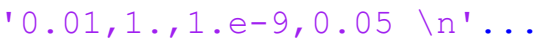

'*boundary $\backslash n$ '...

'global, $3 \backslash n^{\prime} \ldots$

'botnode, 2, , $0 . \backslash n^{\prime}$...

'topnode, $1,, 0 . \backslash n^{\prime} \ldots$

'topnode, $2,1.9 \backslash \mathrm{n}^{\prime}$...

'***boundary, type $=$ displacement $\backslash \mathrm{n}^{\prime} \ldots$

'**top, $1,4 . \backslash \mathrm{n}^{\prime}$...

'*output, field, frequency=1 \n'...

'*Node Output $\backslash \mathrm{n}^{\prime}$...

' $\mathrm{CF}, \mathrm{RF}, \mathrm{U} \backslash \mathrm{n}$ '...

'*Element Output, directions=YES $\backslash \mathrm{n}^{\prime} \ldots$

'LE, PE, PEEQ, PEMAG, S, STATUS, SDEG $\backslash \mathrm{n}^{\prime} \ldots$

'*output, history, frequency=1 \n'...

'*Node Output, nset=top \n'...

'RE2, U2 \n'..

'*Node Output, nset=topnode $\backslash \mathrm{n}^{\prime}$...

'RE2, U2 \n'...

'*Node Output, nset=wavetleft $\backslash n$ '...

'RE2, U2 \n'... 
'*Node Output, nset=wavetmid $\backslash n$ '...

'RE2, U2 \n'..

'*Node print, nset=topnode $\backslash n^{\prime}$...

'RE2, U2 \n'...

'*Node print, nset=wavetleft $\backslash n ' \ldots$

'RF2, u2 $\backslash \mathrm{n}$ '...

'*Node print, nset=wavetmid $\backslash n^{\prime} \ldots$

'RF2, U2 \n'...

'*controls, parameter=field, field=displacement $\backslash \mathrm{n}^{\prime}$...

' $0.01,1 . \backslash n^{\prime} \ldots$

'*END $\mathrm{STEP} \backslash \mathrm{n}$ '...

' '])) ;

fclose (fid);

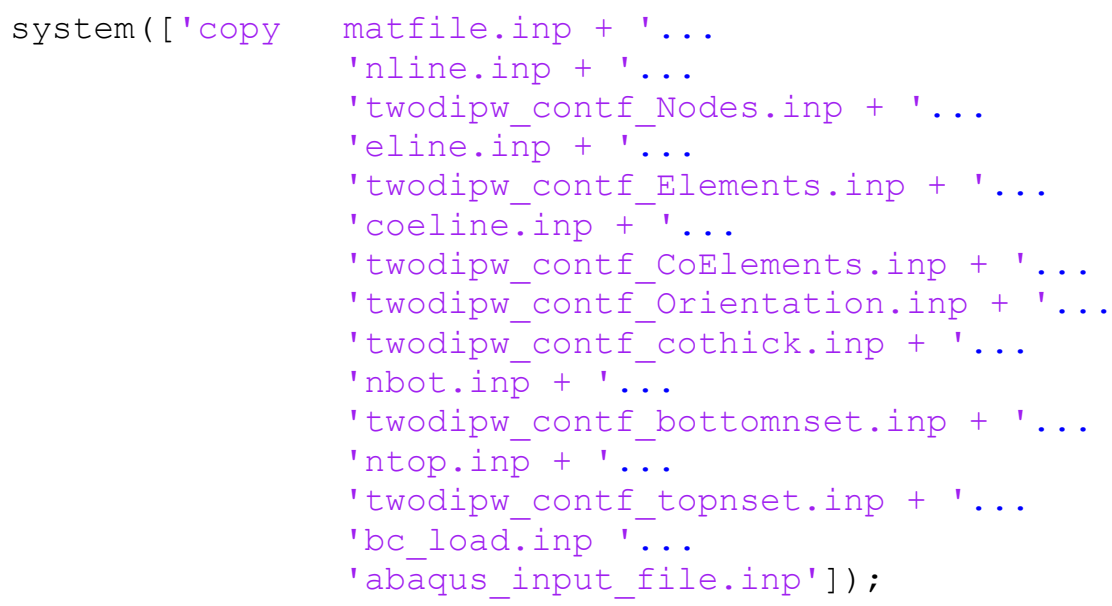

